\title{
Hype or hope? Vitamin D in multiple sclerosis
}

\author{
Citation for published version (APA):
}

Muris, A-H. E. (2016). Hype or hope? Vitamin D in multiple sclerosis: A clinical and immunological perspective. [Doctoral Thesis, Maastricht University]. Maastricht University. https://doi.org/10.26481/dis.20161007am

Document status and date:

Published: 01/01/2016

DOI:

10.26481/dis.20161007am

Document Version:

Publisher's PDF, also known as Version of record

\section{Please check the document version of this publication:}

- A submitted manuscript is the version of the article upon submission and before peer-review. There can be important differences between the submitted version and the official published version of record.

People interested in the research are advised to contact the author for the final version of the publication, or visit the DOI to the publisher's website.

- The final author version and the galley proof are versions of the publication after peer review.

- The final published version features the final layout of the paper including the volume, issue and page numbers.

Link to publication

\footnotetext{
General rights rights.

- You may freely distribute the URL identifying the publication in the public portal. please follow below link for the End User Agreement:

www.umlib.nl/taverne-license

Take down policy

If you believe that this document breaches copyright please contact us at:

repository@maastrichtuniversity.nl

providing details and we will investigate your claim.
}

Copyright and moral rights for the publications made accessible in the public portal are retained by the authors and/or other copyright owners and it is a condition of accessing publications that users recognise and abide by the legal requirements associated with these

- Users may download and print one copy of any publication from the public portal for the purpose of private study or research.

- You may not further distribute the material or use it for any profit-making activity or commercial gain

If the publication is distributed under the terms of Article $25 \mathrm{fa}$ of the Dutch Copyright Act, indicated by the "Taverne" license above, 


Hype or hope? Vitamin D in multiple sclerosis

a clinical and immunological perspective 
Hype or hope? Vitamin D in multiple sclerosis; a clinical and immunological perspective

$\mathrm{PhD}$ thesis with summary in English and Dutch

Copyright (C) Anne-Hilde Muris, Maastricht 2016

ISBN: 978-94-6332-054-2

Keywords: adaptive immune system, auto-immunity, cytokines, disease progression, immune regulation, multiple sclerosis, Th cells, translational research, vitamin $D$, vitamin $D$ supplementation

A digital version of this thesis is available from https://cris.maastrichtuniversity.nl/portal/

Cover: Painting (acrylic on paper) inspired by the Servaasbrug (Maastricht, NL). It represents the bridge between basic and clinical research, which can only be built with a multidisciplinary approach.

The work described in this thesis has been carried out at the Maastricht University Medical Centre (Maastricht, NL) and the Academic MS Centre Limburg (Sittard, NL; www.mscentrumlimburg.nl). Part of the research was financially supported by Merck Serono and the Nationaal MS Fonds.

Financial support for this thesis was kindly provided by the Nationaal MS Fonds. 


\title{
Hype or hope? Vitamin D in multiple sclerosis a clinical and immunological perspective
}

\author{
PROEFSCHRIFT \\ ter verkrijging van de graad van doctor aan de Universiteit Maastricht, \\ op gezag van de Rector Magnificus, Prof. dr. L.L.G. Soete \\ volgens het besluit van het College van Decanen, \\ in het openbaar te verdedigen \\ op vrijdag 7 oktober 2016 om 14.00 uur \\ door
}

Anne-Hilde Elisabeth Muris 


\section{Promotor}

Prof. dr. R.M.M. Hupperts

\section{Copromotoren}

Dr. J.G.M.C. Damoiseaux

Dr. J.J.F.M. Smolders (Canisius-Wilhelmina Ziekenhuis, Nijmegen)

\section{Beoordelingscommissie}

Prof. dr. R.J. van Oostenbrugge (voorzitter)

Prof. dr. A.E.R.C.H. Boonen

Prof. dr. N. Hellings (Universiteit Maastricht/ Universiteit Hasselt, België)

Prof. dr. T. Holmoy (Akershus University Hospital, Noorwegen)

Dr. W.I.M. Verhagen (Canisius-Wilhelmina Ziekenhuis, Nijmegen) 
"Ik word daar enigszins filosofisch van. Dan vraag ik me af: wanneer is iets eigenlijk waar? Als de wetenschap het heeft bewezen? Als genoeg mensen erin geloven? Of als de júiste mensen erin geloven?"

"That makes me somewhat philosophical. Then I wonder: when is something actually true? If science has proven it? If enough people believe in it? Or if the right people believe in it?"

General Zobel in "Duel met Paard" (Duel with Horse), a historical novel written by Paulien Genee. The general is discussing whether or not to start a scientific investigation on the intelligence of Hans, the horse of Wilhelm von Osten. Hans could perform square roots and his owner, Wilhelm von Osten, was inspired by him and hoped to show that animals have intelligence comparable to men. 



\section{Contents}

Abbreviations and glossary

$\begin{array}{ll}\text { General introduction } & 13\end{array}$

Chapter 1. Review on the in vivo immune modulatory effects of vitamin 27

$D$ in auto-immunity, focused on multiple sclerosis

Chapter 2. Vitamin D and disability progression in patients with multiple sclerosis

Chapter 3. Vitamin D and conversion to progressive disease in patients with multiple sclerosis

Chapter 4. Regulation of encephalitogenic GM-CSF producing T cells by vitamin $D$ and regulatory $T$ cells in multiple sclerosis

Chapter 5. Optimisation of the detection of IL-10 producing T cells by flow cytometry

Chapter 6. Immune regulatory effects of high dose vitamin D supplementation in patients with relapsing remitting multiple sclerosis

Summary and general discussion

Nederlandse samenvatting (Dutch summary)

Valorisation

References

Addendum

* Curriculum vitae

* Publications

* Affiliations of co-authors

* Dankwoord/Acknowledgements 



\section{Abbreviations and glossary}

\begin{tabular}{|c|c|c|c|}
\hline $1,25(\mathrm{OH}) 2 \mathrm{D}$ & 1,25-dihydroxyvitamin D & CSF & cerebrospinal fluid \\
\hline$-2 \mathrm{LL}$ & log likelihood ratio & CSIF & cytokine synthesis inhibitory \\
\hline $25(\mathrm{OH}) \mathrm{D}$ & 25-hydroxyvitamin D & & factor \\
\hline 7-DHC & 7-dehydrocholesterol & CTx & C-telopeptide \\
\hline $\mathrm{Ab}$ & antibody & CXCR & C-X-C chemokine receptor \\
\hline AECA & anti-endothelial cell antibody & CYP24A1 & cytochrome P24A1 \\
\hline AI & ambulatory index & CYP27B1 & cytochrome P27B1 \\
\hline AITD & auto-immune thyroid disease & $d$ & day \\
\hline ANA & anti-nuclear antibody & DBP & vitamin $\mathrm{D}$ binding protein \\
\hline APC & antigen presenting cell & DC & dendritic cell \\
\hline ARR & annualized relapse rate & DM & diabetes mellitus \\
\hline ASP & ankylosing spondylitis & DMF & dimethylfumerate \\
\hline B & "from the bone marrow" & DMT & disease modifying treatment \\
\hline BAFF & B-cell activating factor & DNA & deoxyribonucleic acid \\
\hline \multirow[t]{2}{*}{ BAP } & bone-specific alkaline phos- & ds & double stranded \\
\hline & phatase & DSS & disability status scale \\
\hline BBB & blood brain barrier & DT & diphtheria toxoid \\
\hline BCG & $\begin{array}{l}\text { Bycobacterium bovis bacille } \\
\text { Calmette-Guérin }\end{array}$ & $\mathrm{EAE}$ & $\begin{array}{l}\text { experimental auto-immune } \\
\text { encephalomyelitis }\end{array}$ \\
\hline $\mathrm{BD}$ & Behcet's disease & EBV & Epstein Barr virus \\
\hline BFA & brefeldin A & EDSS & expanded disability status \\
\hline \multirow[t]{2}{*}{ BIC } & Bayesian information criteri- & & scale \\
\hline & on & ELISA & enzyme linked immuno- \\
\hline BLG & $\beta$-lactoglobulin & & sorbent assay \\
\hline BMD & bone mineral density & ER & endoplasmic reticulum \\
\hline BMI & body mass index & ergocalciferol & vitamin $\mathrm{D}_{2}$ \\
\hline Breg & regulatory B cell & EX-2 & exon 2 of MBP \\
\hline BSA & bovine serum albumin & ex vivo & "out of the living" \\
\hline C & complement component & $\mathrm{F}$ & female \\
\hline calcitriol & 1,25-dihydroxyvitamin D & FoxP3 & forkhead Box P3 \\
\hline CBA & cytometric bead array & FSC & forward scatter \\
\hline CCP & cyclic citrullinated peptide & GA & glatiramer acetate \\
\hline$C D$ & cluster of differentiation & GAD & glutamic acid decarboxylase \\
\hline cholecalciferol & vitamin $D_{3}$ & $\mathrm{Gd}$ & gadolinium \\
\hline CI & confidence interval & GFAP & glial fibrillary acidic protein \\
\hline CIS & clinically isolated syndrome & GM-CSF & granulocyte macrophage \\
\hline $\mathrm{CL}$ & cardiolipin & & colony-stimulating factor \\
\hline CLIA & $\begin{array}{l}\text { chemiluminescence immuno- } \\
\text { assay }\end{array}$ & GWAS & $\begin{array}{l}\text { genome wide association } \\
\text { study }\end{array}$ \\
\hline CNS & central nervous system & $\mathrm{HC}$ & healthy control \\
\hline CpG & $\begin{array}{l}\text { CpG oligodeoxynucleotide } \\
2006\end{array}$ & hCAP & $\begin{array}{l}\text { human cathelicidin antimi- } \\
\text { crobial peptide }\end{array}$ \\
\hline CRP & C-reactive protein & HLA-DR & human leukocyte antigen \\
\hline CS & casein & & \\
\hline
\end{tabular}




\begin{tabular}{|c|c|c|c|}
\hline \multirow[t]{2}{*}{ htTG } & human tissue transglutami- & NK-T & natural killer-like T (cell) \\
\hline & nase & NMDA & $\mathrm{N}$-methyl-D-aspartate \\
\hline \multirow[t]{2}{*}{ ICAM } & intracellular adhesion mole- & nRNP & nuclear ribonucleoprotein \\
\hline & cule & nTreg & natural regulatory $\mathrm{T}$ cell, \\
\hline IFN & interferon & & derived from the thymus \\
\hline Ig & immunoglobulin & OCB & oligoclonal bands \\
\hline IL & interleukin & OPN & osteopontin \\
\hline IM & intra muscular & OR & odds ratio \\
\hline in vitro & "within the glass" & $\mathrm{p}$ & $p$-value \\
\hline in vivo & "within the living" & PBMC & peripheral blood mononucle- \\
\hline \multirow[t]{2}{*}{ iTreg } & inducible regulatory $\mathrm{T}$ cell, & & ar cell \\
\hline & derived peripherally & PI & proinsulin \\
\hline JCV & John Cunningham virus & PDSS & patient determined disability \\
\hline \multirow[t]{2}{*}{ KIR } & killer immunoglobulin-like & & scale \\
\hline & receptor & pERK & phospho-ERK \\
\hline \multirow[t]{2}{*}{ LAP } & latency activated protein of & PHA & phytohemagglutinin \\
\hline & TGF- $\beta$ & PI & proinsulin \\
\hline M & male & $\mathrm{PL}$ & phospholipid \\
\hline $\max$ & maximum & PLP & proteolipoprotein \\
\hline MBP & myelin basic protein & PMA & phorbol 12 -myristate 13 - \\
\hline \multirow[t]{2}{*}{ MCP } & monocyte chemoattractant & & acetate \\
\hline & protein & PML & progressive multifocal leu- \\
\hline \multirow[t]{2}{*}{ MCTD } & mixed connective tissue & & koencephalopathy \\
\hline & disease & PPD & purified protein derivative \\
\hline MFI & median fluorescence intensity & PPMS & primary progressive multiple \\
\hline \multirow[t]{2}{*}{$\mathrm{MHC}$} & major histocompatibility & & sclerosis \\
\hline & complex & PsA & psoriatic arthritis \\
\hline $\min$ & minimum & Q & quartile \\
\hline MMP & matrix metalloproteinase & $\mathrm{R}$ & receptor \\
\hline \multirow[t]{2}{*}{ MOG } & myelin oligodendrocyte & RA & rheumatoid arthritis \\
\hline & glycoprotein & $\mathrm{RCT}$ & randomised controlled trial \\
\hline \multirow{4}{*}{$\begin{array}{l}\text { monensin } \\
/ \text { monensin }^{+} \\
\text {ratio }\end{array}$} & fraction (or MFI) of cytokine & relapse & exacerbation, period of \\
\hline & positive cells measured in the & & neurological dysfunction \\
\hline & absence of monensin divided & remission & (period of) recovery \\
\hline & $\begin{array}{l}\text { by the fraction (or MFI) in the } \\
\text { presence of monensin }\end{array}$ & REML & $\begin{array}{l}\text { restricted maximum likeli- } \\
\text { hood }\end{array}$ \\
\hline MRI & magnetic resonance imaging & RIA & radioimmunoassay \\
\hline mRNA & messenger ribonucleic acid & Ribo $P$ & ribosomal $\mathrm{P}$ \\
\hline MS & multiple sclerosis & RIS & radiologically isolated syn- \\
\hline \multirow[t]{2}{*}{ MSFC } & multiple sclerosis functional & & drome \\
\hline & composite & RF & rheumatoid factor \\
\hline \multirow[t]{2}{*}{ MSSS } & multiple sclerosis severity & RNA & ribonucleic acid \\
\hline & scale & RNP & ribonucleoprotein \\
\hline $\mathrm{N}$ & number & RPMS & relapsing progressive multi- \\
\hline \multirow[t]{2}{*}{ NAWM } & normal appearing white & & ple sclerosis \\
\hline & matter & RRMS & relapsing remitting multiple \\
\hline NK & natural killer (cell) & & sclerosis \\
\hline
\end{tabular}


SI

SLE

$\mathrm{Sm}$

SNRS

SOLAR

SOLARIUM

SPAG

SPMS

SS-A

SSC

TB

Tc

Tep

TGF

Th

Th1

Th2

TIMP

TLR

$\mathrm{Tm}$

TNF

$\operatorname{Tr} 1$

Treg

Tresp

TT

UVB

VDP

VDR soluble

stem cell transplantation

standard deviation

staphylococcal enterotoxin B

stimulation index (MTT-assay)

systemic lupus erythemato-

sus

Smith antigen

Scripps Neurological Rating

Scale

Supplementation of Vigantol

oil versus placebo as add-on

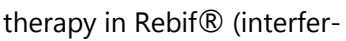

on-beta) treated patients

with relapsing remitting

multiple sclerosis

SOLAR immune modulating

effects

sperm associated antigen

secondary progressive multi-

ple sclerosis

anti-Ro antibody

side scatter

tuberculosis

cytotoxic T cell

trans endothelial protrusion

tumour growth factor

$\mathrm{T}$ helper cell

T helper cell type 1, associated with IFN-y production

$T$ helper cell type 2, associat-

ed with IL-4 production

T helper cell type 17 , associated with IL-17 production tissue inhibitor of metallo-

proteinase

Toll like receptor

time-point measured

tumour necrosis factor

Type 1 regulatory $\mathrm{T}$ cell

regulatory $\mathrm{T}$ cell

responder $\mathrm{T}$ cell

Tetanus toxoid

ultraviolet $\mathrm{B}$ radiation

vitamin $D$ binding protein

vitamin $D$ receptor
VDRE

WBC

Wbp

WGBO

Y vitamin $D$ response element

white blood cell count

wet bescherming persoons-

gegevens (NL)

wet geneeskundige behande-

lingsovereenkomst (NL)

year 

General
introduction 
Vitamin $D$ has gained a lot of attention in different research areas and diseases over the last decades. [1] Talking to people about vitamin D and vitamin D supplementation evokes a number of different responses. Many patients get a sense of hope and the idea of nothing ventured, nothing gained. It gives scientists a great deal of excitement about future prospects and in most doctors it sets alight an enthusiasm to help patients in a simple and affordable way. Else ways, there are also physicians and researchers who have a rather sceptical view on vitamin $D$ presented as the new panacea. [2] These diverse expectations bring together different stories, some of them based on facts, some based on big promises and hope.

This thesis describes the search for some missing pieces in the understanding of the role of vitamin D in multiple sclerosis (MS), focusing on the clinical and immunological effects. It will add information on the underlying etiological mechanism of this still poorly understood disease, by adding knowledge on the associations between vitamin levels and long-term clinical outcomes, and will discuss the immunological effects of high dose vitamin D supplementation as a possible add on treatment in patients with MS.

\section{Multiple sclerosis some time ago}

Times change and so do we. Around 1395, Lidwina, a girl of 15 years old, went skating in Schiedam (the Netherlands). She fell, broke a rib and since then never recovered (Figure 1). Till her dead in 1433 she experienced a plethora of symptoms and developed a gait disorder, paresis and blindness, suggested to be transient. Due to the way she accepted her illness, performed prayers and mental exercises and even wanted to suffer more to release spirits from the purgatory, made her to be canonized. She became the Saint of the city of Schiedam in 1890, now 125 years ago. Looking back, Lidwina is possibly the first described case of a person with MS. [3, 4]

Since then, great efforts have been made to describe, understand and treat this disease more accurately. Among others, at the end of the 18th century, Charcot and his students made great contribution to the concept of MS by being the first to distinguish MS as a separate neurological entity. They were able to correlate clinical and pathological findings and to describe mild and benign forms of MS. Furthermore they were the first who spoke about "sclerotic patches", referring to the multifocal inflammation within the central nervous system (CNS), forming scars and resulting in failure of neurological function. [5, 6] Halfway the $20^{\text {th }}$ century, the development of an experimental auto-immune encephalitis (EAE) animal model [7-9], further increased the progression in the understanding of the disease and in the development of therapies. 


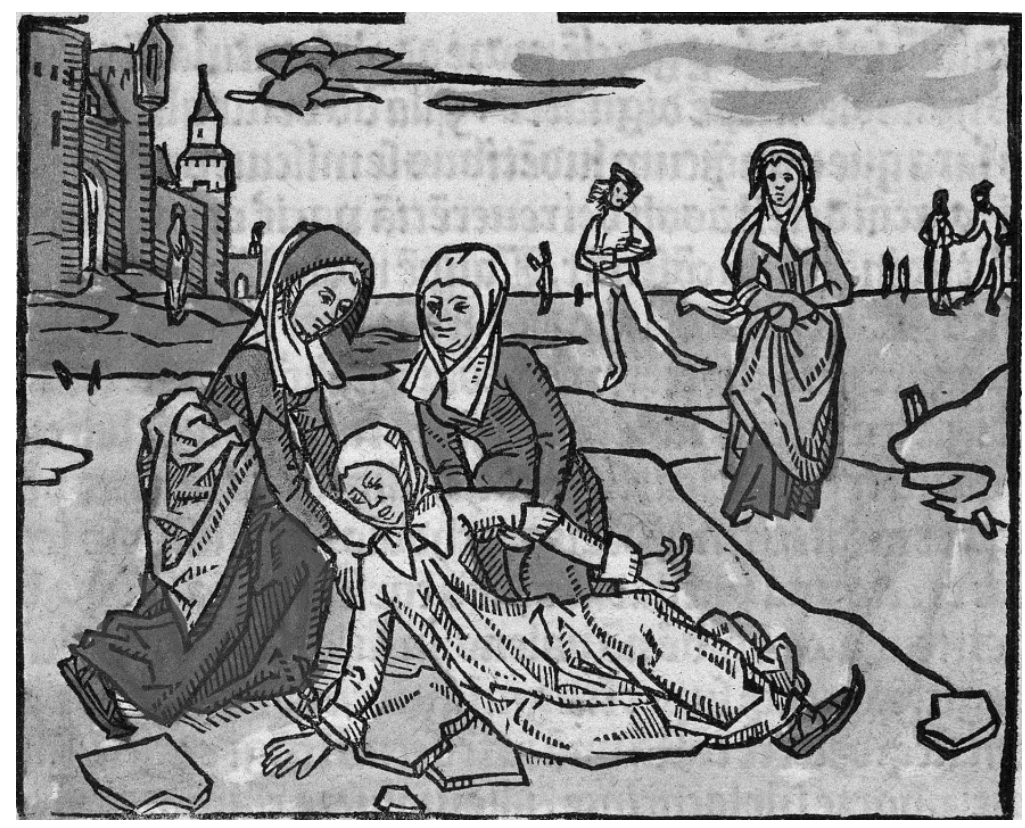

Figure 1. Woodcut "Vita alme virginis Lijdwine" (Johannes Brugman, Otgier Pietersz. Nachtegael, 1489, collection Museum Catharijneconvent, Utrecht, the Netherlands).

\section{Current insights in multiple sclerosis}

\section{Multiple sclerosis, some general information}

Today, MS is defined as an inflammatory disease of the CNS. This disease affects approximately 16.000 people in the Netherlands and at least 1.3 million people worldwide $[10,11]$, women being at least 2 times more prone to get the disease than men [10]. The onset of the disease is usually between 20-40 years of age. The exact aetiology of the disease is not known. However, it is clear that a combination of genetics and environmental risk factors play a role, with a substantial overlap of these causative factors with other auto-immune diseases. [12] Presumably, individuals who are susceptible for a dysregulation of the immune system, caused by Major Histocompatibility Complex (MHC) related genotypes (especially DRB1*1501) [13], in combination with several genetic polymorphisms in for example the interleukin 7 receptor (IL-7R) gene or the IL-2R gene [14-16], and the influence of environmental factors, like smoking or low vitamin $D$ levels, will develop an auto-immune disease after an initiating trigger. This trigger will then easily disrupt immune homeostasis resulting in loss of self-tolerance. The exact origin of this initiating trigger is unknown, but an infection with the Epstein Barr virus (EBV) $[17,18]$ or endogenous factors as stress, are possible candidates. 


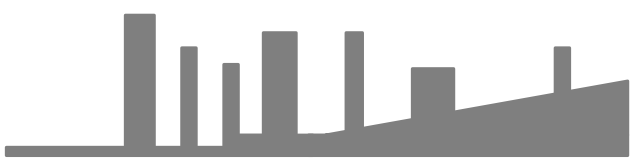

\section{Clinical signs}

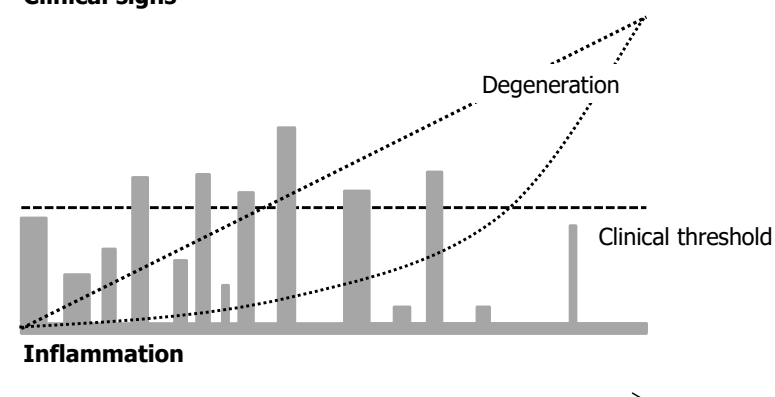

Time

B

PPMS

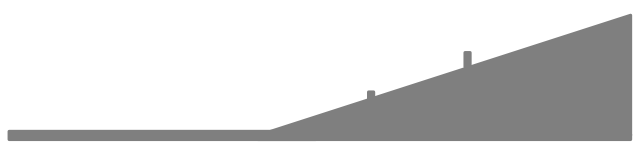

\section{Clinical signs}

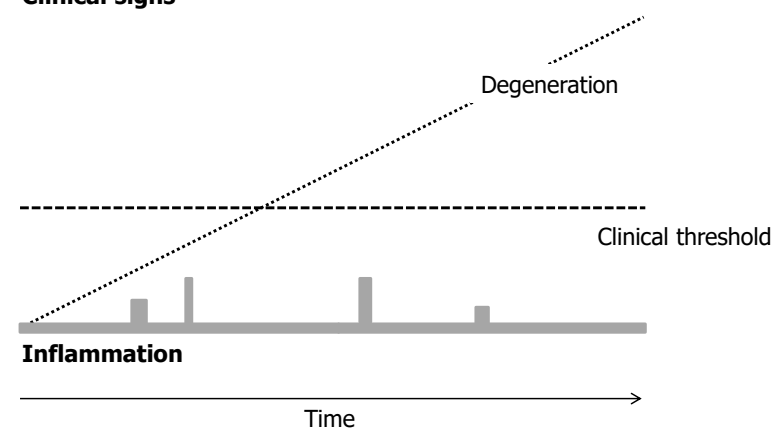

Figure 2. The pathophysiological mechanism and clinical course in (A) RRMS and SPMS and (B) PPMS.

The upper graphs demonstrate clinical symptoms; the lower graphs show the underlying pathophysiological processes of inflammation and degeneration.

In relapsing remitting multiple sclerosis (RRMS), inflammatory activity and possible thereto linked degeneration might precede the actual clinical symptoms. In the auto-immune hypothesis this degeneration is considered to be a consequence of inflammation. In the cytodegeneration hypothesis it is considered to be the primary cause. Over time, degeneration will (exponentially or linearly) become more prominent and the disease will progress between relapses entering the secondary progressive phase (SPMS).

In primary progressive multiple sclerosis (PPMS), there is a significant degeneration without preceding inflammatory phases in most of the patients. Symptoms are observed from the moment compensatory mechanisms fail to counterbalance the degenerative effects. Therefore the onset of PPMS is usually at a later age. 
The most common phenotypic entity of MS is the so called relapsing remitting MS (RRMS) subtype, characterized by periods of neurological dysfunction (relapses) followed by recovery (remission). Symptoms can be diverse, but include tingling (paraesthesia) and numbness, cognitive dysfunction, depression, fatigue, muscle spasms, weakness, walking difficulty, dizziness, vision problems, pain, and bladder and bowel dysfunction. In most patients the recovery between relapses will be complete in the first years but usually becomes incomplete later on. From the moment neurological dysfunction gradually worsens between relapses, the disease has entered a progressive state and is called relapsing progressive MS (RPMS) or secondary progressive MS (SPMS). A minority of patients has progressive disease from onset (without relapses), which is called primary progressive MS (PPMS). [19] (Figure 2)

Diagnosis has long been made purely on clinical presentation based on the criteria of Schumacher [20] and Poser [21]. The most important principle is to establish the dissemination in time and space of the MS lesions. This means that for the diagnosis of RRMS at least two relapses have to be objectified, with involvement of different systems within the CNS, at least 30 days apart. Magnetic resonance imaging (MRI) can nowadays be used to confirm or prove one of these relapses by showing gadolinium $(\mathrm{Gd})$ enhancing brain or spinal cord lesions, or new T2 lesions. PPMS can be diagnosed after 1 year of progressive symptoms and brain and/or spinal cord involvement on MRI or oligoclonal bands (OCB) in the cerebrospinal fluid (CSF). [22-24] Apart from these MS subtypes, a clinically isolated syndrome (CIS) can be distinguished. This syndrome is characterized by a single attack of neurological function loss, suggestive of MS. Depending on the genetic background and the presence of environmental factors, thirty to seventy percent of CIS patients will develop MS later in life. [25] Finally, MRI hallmarks typical for MS without any clinical signs are described as radiologically isolated syndrome (RIS). [26]

\section{Multiple sclerosis as an auto-immune disease}

It is not clear yet whether the different subtypes of MS are presentations of the same underlying disease or from different diseases. What is evident is that MS symptoms are caused by a combination of inflammation and degeneration within the CNS. The prototypic example of inflammatory MS is early RRMS. Later during RRMS and in progressive disease, inflammation becomes less prominent and degenerative aspects are more outstanding. The pathophysiological hypothesis most widely accepted is that of MS being an auto-immune disease. Autoimmune diseases result from an error in the immune system that hampers appropriate discrimination of self and non-self, resulting in a deleterious response to self-antigens. Although the causative agent and the exact auto-antigen in MS are unknown, the auto-immune hypothesis supposes naïve $T$ and $B$ cells to be primed in the periphery with CNS antigens. These antigens can enter the vascular system through either leakage of CNS proteins from the brain or by molecular mimicry with a foreign antigen. [27] Molecular mimicry refers to a shared structural, functional or immunological macromolecule between two different entities, for example an infectious pathogen and its host tissue. In humans, EBV proteins have been shown to have overlap with human myelin associated proteins. [28, 29] After priming in 
secondary lymphoid tissues, $\mathrm{T}$ and $\mathrm{B}$ cells enter the CNS via the blood brain barrier (BBB), where they re-encounter the specific antigen and become reactivated. This leads to a proinflammatory environment within the CNS, an increased cytokine production and attraction of many other kinds of immune cells. As a consequence, myelin sheets and neurons are damaged, eventually resulting in demyelination, the typical hallmark of MS. The main function of myelin is to synchronize afferent nerve signal input. It is necessary for a fast and fluent signal transduction from the cells of the CNS into the periphery. These functions will be affected in MS patients and lead to the characteristic symptoms. During the disease course, loops of demyelination and remyelination will ultimately lead to astrocytosis and if the disease becomes progressive, axon degeneration will accumulate.

Several lines of evidence underpin that MS is an auto-immune disease. MS is associated with auto-antibodies. Auto-antibodies to a whole array of myelin-associated proteins are detectable in MS patients, such as myelin basic protein (MBP), proteolipoprotein (PLP), and myelin oligodendrocyte glycoprotein (MOG), but also more recently discovered auto-antigens against non-myelin antigens as $\alpha B$-crystalline [30], KIR4.1 [31] and SPAG16 [32]. They can be shown intrathecally, as OCBs in the CSF, or in the blood. The role of these auto-antibodies in the pathogenesis is not clear. They probably contribute to the disease process, but they are not single-actors inducing disease, as they can also be shown in healthy controls (HC). [33] The role of immunoglobulins or other serum components in the pathophysiology is also supported by the effectiveness of plasma exchange, when the blood plasma is replaced by donor plasma or replacement fluids. Furthermore, MS is considered to be a T-cell mediated auto-immune disease. Both T helper (Th) cells and cytotoxic T (Tc) cells are assumed to play a role as initiators of disease or as effectors. They can be found at the sites of inflammation, together with macrophages and B cells. In early RRMS, the circulating immune cell compartment outside the CNS is characterized by a decrease in the function of regulatory cells and a shift to a more pro-inflammatory immune cell profile. [34, 35] For many years, MS was regarded to be a T helper 1 (Th1; mainly IFN $-\nu$ producing Th cells) mediated disease. [36] More recently, Th17 cells, producing interleukin 17 (IL-17) [36, 37], and GM-CSF producing T cells $[38,39]$ are shown to have strong encephalitogenic capacities and are now thought to be the 'pathogenic' Th cells in MS. Regulatory T cells are important to keep proinflammatory cells in control. Also genome wide association studies (GWAS) have supported MS to be a T cell dependent disease. [14]

Transfer of the disease to healthy individuals or animals is one of the other criteria for an auto-immune disease defined in the (revised) Witebsky's postulates. [40] However, in MS, it is difficult to prove this, because T cells do not efficiently cross the placenta, and therefore neonates of mothers with MS are not affected. Also passive immunization with auto-reactive $\mathrm{T}$ cells from humans to animals is not possible because of $\mathrm{MHC}$ restriction in antigen recognition by T cells. [41] On the other hand, the EAE model, induced by immunization of rodents with CNS homogenate or (parts) of CNS proteins, can be adoptively transferred by autoreactive $T$ cells to healthy syngeneic recipients. [42] Also the arguments that the disease is 
most prevalent in young women, that the disease course is relapsing remitting and that immune modulating drugs have a favourable response, all give circumstantial evidence for MS to be an auto-immune disease.

\section{Alternative pathophysiological hypotheses}

Several researchers have come up with alternative hypotheses for the pathophysiology in MS. An interesting alternative hypothesis is that of MS being the consequence of progressive cytodegeneration. [43] In contrast to the auto-immune hypothesis, the basis of this hypothesis is that degeneration of presumably oligodendrocytes and myelin provokes the disease development and becomes very quickly intertwined with a disbalanced immune system. Evidence from different approaches could support this hypothesis. Pathological studies have shown that the earliest histological changes occur at the inner layer of the myelin sheet, while the outer myelin wrap is still intact. Moreover, in early MS brain sections mainly macrophages are abundantly present, which might indicate that the digestion of debris occurs before the actual activation of T and B cells. In normal appearing white matter (NAWM) in the CNS of MS patients, signs of myelin and axonal degeneration have been observed, with little evidence for involvement of an adaptive immune response. Disease modifying treatments (DMT) have shown effects on the immune system, but are not able to hold the progression of demyelination, axonal degeneration and brain atrophy. This makes MS progression largely independent of inflammatory relapse activity. According to the cytodegeneration hypothesis, the different subtypes of MS could be explained by a difference between the genetic make-up of individuals in the way their immune system reacts to the initial cytodegeneration. Underlying mechanisms for this cytodegeneration could be an over-activation of the NMDA receptor (whether or not caused by a dysregulation in the copper homeostasis), myelinopathy or an over-activation of other glutamate receptors.

Another alternative hypothesis focusses more on the role of astrocytes versus microglia as antigen presenting cells in the CNS which induce the reactivation and pathogenicity of autoreactive $T$ cells. Also different vascular components have been under investigation: MS patients have a higher risk of ischemic stroke, global cerebral hypoperfusion and a slower cerebral venous blood flow and this might point towards a causal component within the vascular system, but the exact mechanism behind this, is not completely clear yet. [44-46]

Overall, independent of the most plausible pathophysiological hypothesis, it is clear that inflammation is the most important provoker of symptoms in early RRMS, while symptoms in later RRMS and progressive disease are the result of an outstanding degenerative component (Figure 2).

Within this framework, it is interesting to mention that the CNS has for a long time been seen as an immune privileged structure, without surveillance of immune cells in a healthy situation. However, it has now become clear that immune cells can cross the BBB in healthy and diseased individuals. [47, 48] Moreover, very recently, two papers $[49,50]$ have been published describing a lymphatic system in the CNS suggestive for a possible route for antigen leakage into the cervical lymph nodes. 


\section{Current and upcoming treatment options in MS}

MS is as yet an incurable disease. Treatment focuses on the prevention of relapses and disability progression and is three part. [51] First, acute relapses are treated with intravenous corticosteroids or in severe cases with plasma exchange. Second, multidisciplinary symptom management is needed to promote the functional abilities, independence and the quality of life of the patient. Third, since the end of the 20th century, drugs that interfere with the disease process, the DMTs, have become available. In general, these therapies affect the inflammatory component of the disease and are therefore mainly applicable for CIS, RRMS and sometimes for SPMS patients, if still suffering from relapses. They mainly affect the disease activity, not so much the disability progression. The most well-known and safe DMTs are IFN- $\beta$ and glatiramer acetate (GA). Recently, oral first-line therapies with dimethylfumerate (DMF) and teriflunomide have been approved. Second line treatment consists of fingolimod, natalizumab or alemtuzumab. Drugs currently in development for MS treatment include variants of IFN- $\beta$ (NU100 (NCT01464905) [52] and Bii-B017 (NCT01332019)) and natalizumab (firategrast [53]) and drugs with different modes of action like daclizumab [54] and laquinimod [55]. Also B cell depleting therapies as rituximab/ocrelizumab/ofatumumab [56] look very promising. Hematopoietic stem cell transplantation [57] still awaits any clinical breakthrough. In the future, anti-LINGO1 [58], rHigM22 (anti-oligodendrocyte IgM) [59] and possibly also stem cell therapies, may affect regeneration, remyelination and survival of CNS cells, making these drugs particularly interesting for the treatment of progressive patients. There is currently no evidence for combination therapies in MS.

DMTs exert their disease modifying actions by affecting different steps in the immunological cascade of the disease process. First, some drugs (like DMF [60], teriflunomide [61], fingolimod [62], alemtuzumab [63] and daclizumab [54]) decrease the amount of lymphocytes in the circulating immune cell compartment, thereby decreasing the number of circulating potentially pathogenic cells. Second, the balance between pro- and anti-inflammatory cytokines and cells, which is shifted to a more pro-inflammatory profile in patients with MS, can be rebalanced by either increasing the anti-inflammatory compartment, decreasing the proinflammatory compartment or by increasing the number or function of regulatory cells (e.g. DMF [60, 64], GA [65]). A third mode of action is to decrease the activation of proinflammatory and probable pathogenic $T$ cells for example by decreasing antigen presentation (e.g. IFN- $\beta$ [66] and rituximab [56]). Fourth, the ability of activated pro-inflammatory and pathogenic cells to cross the BBB can be blocked or decreased, by blocking $T$ cell adhesion molecules or proteases needed for crossing (e.g. IFN- $\beta$ [66], natalizumab [67]). Fifth, drugs may have neuroprotective effects (possibly DMF [60, 64] and fingolimod [62]). The last option is to enhance regeneration and survival of CNS cells. All DMTs have minor to even major and severe side effects, making patients more vulnerable to infection. The blockade of immune cells entering the brain, by in particular natalizumab, but also after use of fingolimod and DMF, may even induce progressive multifocal leukoencephalopathy (PML), a severe and serious side effect, in anti-JC-virus antibody positive patients. 


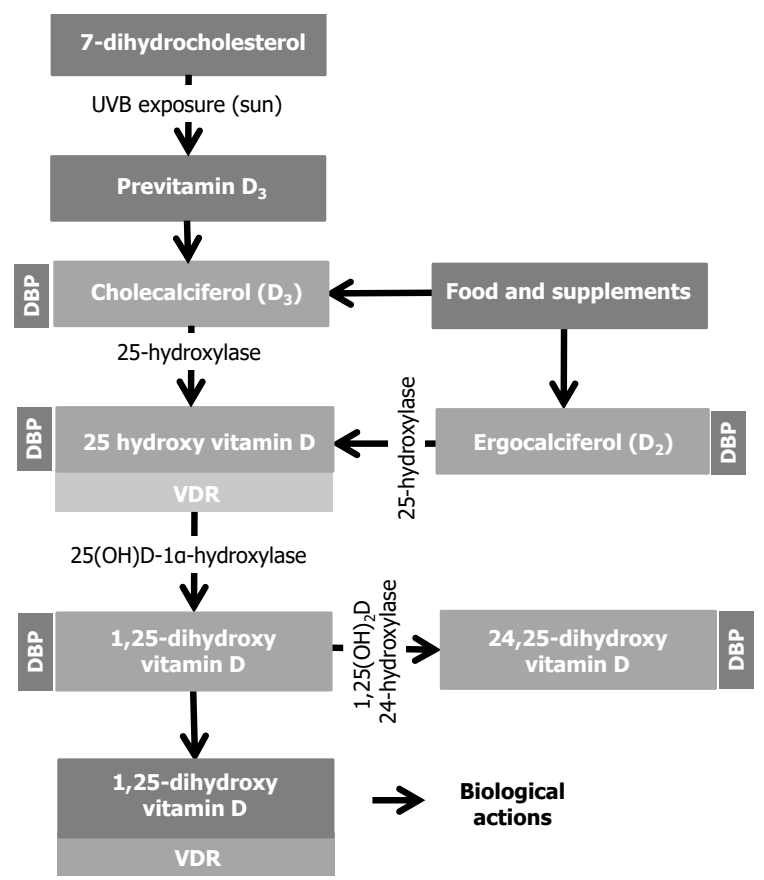

Figure 3. Schematic overview of vitamin D metabolism.

Several metabolites of vitamin $D$ can be found in the circulation: cholecalciferol (vitamin $D_{3}$ ), ergocalciferol (vitamin $\left.\mathrm{D}_{2}\right), 25(\mathrm{OH}) \mathrm{D}$ and $1,25(\mathrm{OH})_{2} \mathrm{D}$, either free or bound to the vitamin $\mathrm{D}$ binding protein (VDP).

$25(\mathrm{OH}) \mathrm{D}$ is the metabolite that is measured to assess the vitamin $\mathrm{D}$ status.

Via the activation of the vitamin D receptor (VDR) by $\left(25(\mathrm{OH}) \mathrm{D}\right.$ and) $1,25(\mathrm{OH})_{2} \mathrm{D}$, vitamin $\mathrm{D}$ can exert its biological actions.

$D P B$ : Vitamin D binding protein, VDR: vitamin D receptor

\section{The role of vitamin D in multiple sclerosis}

Vitamin $D$ is an essential vitamin, especially for bone metabolism. Humans obtain vitamin D to the largest extend (90-100\%) via sun exposure. A small part is obtained from food, both from animal origin (cholecalciferol, e.g. fatty fish), and vegetable origin (ergocalciferol, e.g. mushrooms). Upon UVB light exposure, 7-dihydrocholesterol in the skin is converted to previtamin $D_{3}$ and enters the blood stream. In the liver this is converted to 25 -hydroxy vitamin $D$ $(25(\mathrm{OH}) \mathrm{D})$, the vitamin $\mathrm{D}$ metabolite we measure in the blood to estimate vitamin $\mathrm{D}$ levels. $25(\mathrm{OH}) \mathrm{D}$ is an inactive metabolite, but it is converted to the active metabolite 1,25-dihydroxy vitamin $D\left(1,25(\mathrm{OH})_{2} \mathrm{D}\right)$ in kidneys and other tissues expressing $25(\mathrm{OH}) \mathrm{D}-1 \alpha$ hydroxylase (CYP27B1), like immune cells and brain tissue. This metabolite is catabolized by $1,25(\mathrm{OH})_{2} \mathrm{D}$ 24-hydroxylase (CYP24A1) to 24,25-dihydroxy vitamin D. 1,25(OH) $)_{2} \mathrm{D}$ performs its actions via the activation of the intracellular vitamin $D$ receptor (VDR) which regulates the expression of more than 500 genes (Figure 3). 


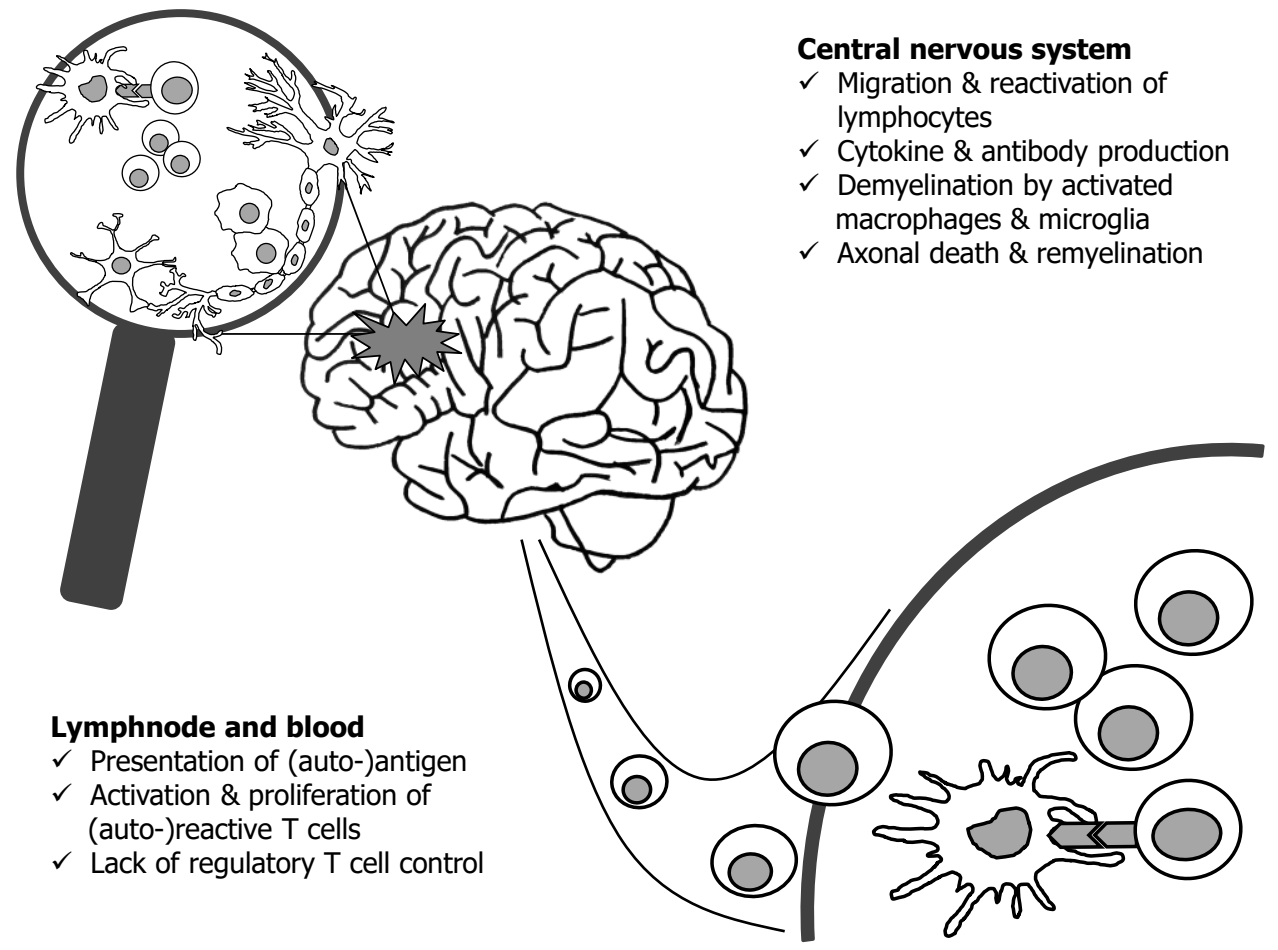

Figure 4. Steps in the MS disease process and potential targets for vitamin D on the cells of the circulating immune compartment and/or on cells of the CNS.

Naïve T and B cells are primed, via antigen presenting cells, with CNS antigens. They enter the CNS via the blood brain barrier (BBB), where they re-encounter the specific antigen and become reactivated. In the proinflammatory environment that arises within the CNS, myelin sheets and neurons are damaged eventually resulting in demyelination, the typical hallmark of MS.

Adapted from Smolders J., Hupperts R. and Damoiseaux J. (2016). [85]

Sufficient levels of vitamin D are needed for adequate calcium regulation and bone metabolism. But also the risk of the development of auto-immune diseases as MS has been associated with insufficient sun exposure and low vitamin D levels. First of all, the geographical variation in the prevalence of MS, with a higher occurrence in northern latitudes and a lower occurrence in people living around the equator, is well proven. [68] Secondly, compared to the general population, an increased proportion of patients with MS was born in the months after the winter season [69] and vitamin D levels have been associated with MS risk in large prospective cohort studies. [70] Next to this, low vitamin D levels have been associated with disease activity in patients with MS. Low vitamin D levels are associated with an increased relapse risk with a decrease in the risk of $9-34 \%$ with each $10 \mathrm{nmol} / \mathrm{L}$ increase in $25(\mathrm{OH}) \mathrm{D}$ levels. [71-74] These findings led to the hypothesis of vitamin $D_{3}$ supplementation to be beneficial in RRMS patients, an assumption that has already been investigated in some small 
high dose supplementation studies. [75-81] These studies were severely underpowered to detect a clinical meaningful effect, but most of them showed high dose vitamin D supplementation to be safe and suggested a favourable effect of supplementation on MS disease activity.

The underlying favourable mechanism is supposed to be the immune regulatory effect of vitamin D. This effect could either be in the circulating immune cell compartment or in the CNS itself (Figure 4). Immune cells are able to metabolize vitamin D and in vitro and in vivo studies show that vitamin $D$ increases regulatory $T$ cell (Treg) function and shifts the balance between pro- and anti-inflammatory immune cells. This will be described more in detail in chapter 1. Cells of the CNS, like neurons, microglia and astrocytes have been shown to express the VDR and to have functional VDR signalling. [82] Moreover, expression of the key proteins in vitamin $D$ metabolism was increased in MS chronic active lesions compared to NAWM. [82] Vitamin D within the CNS might be beneficial by reducing the inflammatory profile of CNS cells, increasing neurothrophin production, modulating $\mathrm{Ca}^{2+}$ homeostasis or inhibiting nitric oxide synthase leading to a neuroprotective effect. [83, 84] The effects on demyelination and remyelination are, however, poorly investigated.

\section{Evaluating the vitamin $D$ effects in multiple sclerosis}

The effect of vitamin D, vitamin D supplementation and other potential therapeutic strategies in MS can be evaluated either with clinical outcome measures or MRI, which are important for neurologists and patients, or by assessing the effects on the immune system, to further unravel the underlying mechanisms.

Clinical outcomes can be measured by the assessment of biological disease activity, i.e. relapse rate in RRMS patients. A relapse is defined as the development of new symptom(s) or aggravation of existing symptom(s) for at least 24 hours in a patient with stationary or improving disease course in the previous month. [20] The relapse rate forms one of the most clinical relevant aspects in RRMS patients, is easy to quantify and can be combined with (objective) measures as MRI. However, the relationship between relapses and long-term disability is still uncertain. Another outcome that may be used in very early MS is the conversion from CIS (or RIS) to clinically definite MS.

To assess disability during the disease course several measures can be used. The first method to investigate disability is the expanded disability status scale (EDSS). This is a scoring system from 0 till 10 with steps of 0.5 point, scoring subcategories on eight functional systems: visual (optic) functions, brainstem functions, pyramidal functions, cerebellar functions, sensory functions, bowel and bladder functions, cerebral functions and ambulation. The EDSS has two important cut-off points: below a score of 4, a patient is fully ambulatory and above a score of 5.5, a patient has difficulties to ambulate and needs an aid or use a wheelchair to do so. The EDSS is the score most often used in clinical trials, but it has the disadvantages that it is complicated to score, that the low end of the scale is rather subjective, 
that it has a poor interrater and test-retest reliability and that the increment per unit on the scale is non-linear. [51] Researchers and clinicians have tried to improve this scoring system by redesigning the score and by introducing alternatives. The MS severity scale (MSSS) takes into account the time that a patient has a certain rate of disability, making it possible to estimate disability progression based on a single assessment. [86] Other less adopted options to assess patient functionality are the area under the curve or integrated DSS [87, 88], the Scripps neurological rating scale (SNRS) [89], the ambulatory index (AI) [90] or the MS functional composite (MSFC) [91]. Other alternative outcome measures could be the evaluation of (grey matter) atrophy on MRI [92-95] or evoked potentials of visual, motor and/or somatory sensory systems, which have been implicated for objectivation of MS disease course and have been correlated with MS disability. [96, 97] Another way to measure disability and disability progression is the time to conversion from RRMS to SPMS. This conversion is not so clearly defined in literature and has to be made in retrospect.

Also MRI is a useful outcome measure. The first MRI of a patient with MS was published in 1981 [66], and enormous steps have been made to improve this imaging technique in the following years. Nowadays, (conventional) MRI takes into account the number and volume of T1 and T2 lesions and new T2 gadolinium enhancing lesions. This last parameter is used as an indicator of recent inflammation. MRI outcomes are beyond the scope of this thesis.

Finally, also biomarkers could give information on disease activity or disease progression and could therefore be used to evaluate vitamin D treatment. Biomarkers could include markers of inflammation, demyelination, oxidative stress, glial dysfunction, remyelination and axonal damage. Multiple biomarkers have been under research but only few of them may be suitable to be used for the evaluation of disease prognosis or treatment. [98] Effectiveness of treatment could be investigated either by the use of biomarkers predicting treatment nonresponse (neutralizing antibodies) or by biomarkers predicting adverse effects (like anti-JCV antibodies). [99]

A more mechanistic evaluation of therapy can be obtained by looking at the cells in the circulating immune compartment. This can enhance the understanding of the underlying mechanism of vitamin D in MS.

For a detailed description of immunological parameters, see chapter $\mathbf{1}$ of this thesis. In short, general inflammatory markers in the circulation can be assessed, like serum cytokine and chemokine concentrations. Furthermore auto-antibodies in serum can be determined, but the absence of a MS specific auto-antibody that is detectable in the majority of patients makes this difficult. A disadvantage of measuring serum concentrations is that the cellular source is uncertain and then the question remains what happens at the tissue level. Therefore, one could also assess cytokine excretion in the supernatant of stimulated cell cultures (stimulated either polyclonally or antigen specific) by using ELISA/CBA or Luminex technologies. The induction of proliferation after supplementation with vitamin $D$ can be assessed by labelling cells with a dye like CFSE. It is also possible to phenotypically assess cells directly ex vivo by isolating peripheral blood mononuclear cells (PBMC) and performing flow cytometric 
analyses. The nature of each cell can be assessed based on the surface markers and the proportion of inflammatory and regulatory lymphocyte subsets can be determined. Regulatory $T$ cells can be subdivided in natural Treg ( $\mathrm{nTreg}$ ) which are derived from the thymus and inducible Treg (iTreg) which are derived peripherally. [100]

\section{Vitamin D and MS anno 2016: high dose supplementation studies}

The increasing awareness of the potential role of vitamin D in MS has inspired several research groups around the world to study this phenomenon in depth. A lot of research has been performed, investigating the role of low vitamin D levels in MS risk and disease activity. However, an important gap in our knowledge is the role of vitamin D in disease progression, which is one of the most important hallmarks of disability and has major impacts on the quality of life. Especially progressive patients for whom no treatments are available up to now, and their treating physicians, have interest in an answer to this question. Results on two studies on this subject are described in this thesis (chapter $\mathbf{2}$ and $\mathbf{3}$ )

The second, and maybe most apparent problem in vitamin D research, has been the question whether low vitamin D levels in MS patients are the cause or the consequence of the disease, as we will elaborate on in the discussion of this thesis. The only way to answer this question is by performing well powered randomised placebo controlled trials (RCT). Several of these trials have been initiated. One of these trials initiated in the Netherlands, is the SOLAR trial, in which the effect of vitamin D supplementation as add on therapy in IFN- $\beta$ treated RRMS patients is investigated. In this thesis the results of the SOLARIUM study, the Dutch sub study of the SOLAR trial, will be described. This study investigated the immune regulatory effect of the high dose vitamin D supplementation (chapter 6). 


\section{Aims and outline of this thesis}

The overall hypothesis of this thesis is that vitamin D acts as an immune regulator, decreasing the pro-inflammatory milieu in MS, while preserving the normal immune surveillance. Next to this, vitamin D may also be implicated in disability progression and act directly on CNS tissue, thereby increasing the opportunities to regulate the inflammatory milieu in the CNS, as target organ of the disease, and to affect regeneration.

In chapter 1 we give an overview of the in vivo effects of vitamin D on the immune system, which were shown in cross sectional and longitudinal studies and in small RCTs at the start of the research described in this thesis. Then, with the assumption that vitamin $D$ not only acts as an immune modulator of the circulating immune cell compartment, but also could have local anti-inflammatory effects within the CNS, we assess the relation between vitamin D levels and progression of disease. In chapter $\mathbf{2}$ we investigate whether vitamin D levels during the disease course are associated with disability and disability progression, especially in progressive MS patients. In this study we use the EDSS score to assess disability. In the next chapter, chapter 3, we use an alternative outcome measure of disease progression and share our work on the relation between vitamin D levels at diagnosis and the time to conversion to progressive disease in RRMS patients.

Then we move to the mechanistic site of vitamin $D$ actions. To answer the key question in the relation between vitamin D and disease activity, the SOLAR study was initiated, which is not in the scope of this thesis. In the SOLARIUM sub study we investigate the immune modulatory effects of vitamin $D$ after high dose vitamin $D$ supplementation. In order to use the most relevant outcome measures, we first had to optimize our protocols. GM-CSF, a recently discovered and potential pathogenic cytokine produced by $T$ cells in $E A E$, was not investigated so far in MS patients. Therefore we first wished to increase our knowledge on the role of this cytokine in MS. We wanted to know whether the proportion of T cells producing this cytokine was increased in the blood and CSF of patients with MS and whether it could be regulated by regulatory T cells and vitamin D. In chapter 4 we describe the results of this study in a cross sectional cohort of patients with MS. Furthermore, chapter $\mathbf{5}$ describes the optimisation of the protocol to detect immune cells secreting the cytokine IL-10. IL-10 is a prototypic anti-inflammatory cytokine that is the phenotypic hallmark of inducible Tregs and regulatory B cells (Breg). We were able to improve the protocol and make IL-10 producing cells easier to detect, which is an important step forward to assess regulatory cells. Chapter 6 involves the most important part of this thesis describing the immune regulatory effect of high dose vitamin D supplementation. This thesis will close with a summary and general discussion. 


\section{Chapter}

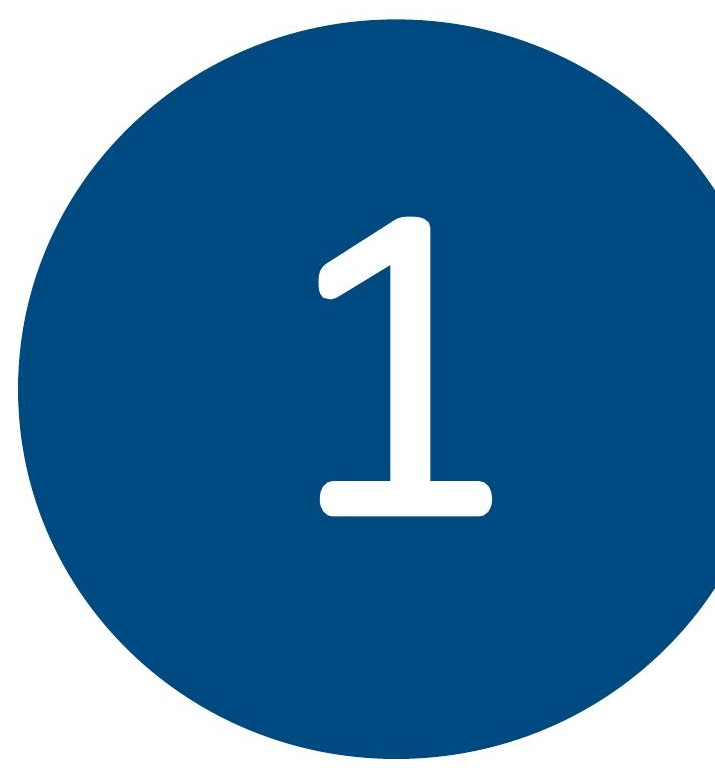

\section{Review on the in vivo immune modulatory effects of vitamin D in auto-immunity, focused on multiple sclerosis}

Published as: Monitoring in vivo immune modulation by vitamin $D$ in multiple sclerosis Muris, A.H., Damoiseaux, J., Smolders, J.; Handbook of Vitamin D in human health: prevention, treatment, and toxicity, Wageningen Academic Publishers, the Netherlands, 2013 


\begin{abstract}
Multiple sclerosis (MS) is an inflammatory, demyelinating disease of the central nervous system, characterized by an auto-reactive $T$ cell response. High serum 25hydroxyvitamin D (25(OH)D) levels have been associated with a decreased risk of developing MS and less severe disease. It is hypothesized that the underlying mechanism behind this association is the immune modulating effect of vitamin $D$ on the adaptive immune response. In vitro studies and in vivo studies in the experimental autoimmune encephalomyelitis (EAE) animal model have consistently shown that immune cells are functional targets of vitamin D. However, in vivo studies in humans are limited. In this chapter, studies on the relation between vitamin $D$ and immunological parameters in healthy individuals and patients with different auto-immune diseases, including MS, are evaluated. Correlation studies and supplementation studies performed thus far are heterogeneous in many aspects and studied a diverse repertoire of immunological outcome measures. Overall, there seems to be a trend towards an antiinflammatory role of vitamin $D$, but this impression is not substantiated by conclusive data. Well-powered and controlled studies assessing disease-specific relevant immunological outcomes are warranted before conclusions can be drawn. Clinical trials on vitamin D supplementation in MS and other diseases may provide excellent frameworks for such studies.
\end{abstract}




\section{Introduction}

Since the beginning of the $20^{\text {th }}$ century vitamin $D$ has been particularly known for its function on bone and calcium metabolism. However, since the seventies, a poor exposure to vitamin $\mathrm{D}$ has also been increasingly noticed in and linked to the aetiology of multiple sclerosis (MS). [101, 102] Since then, a new research field developed, addressing the implications of vitamin D in the pathophysiology of MS in epidemiological studies, intervention studies, in vitro studies and animal studies. [103] Currently, almost 50 years later, first randomised controlled clinical trials are assessing the efficacy of vitamin D supplementation in MS. The view of vitamin $D$ changed from that of a vitamin with a single role in calcium homeostasis, to that of an exciting steroid hormone involved in a multitude of cellular processes. One of these processes is the regulation of the adaptive immune response, with many in vitro and animal studies showing important regulatory effects of vitamin D. [104, 105] One of the major challenges that lies ahead is to understand if and how systemic vitamin D status interferes with the human adaptive immune response in vivo. Clarification of the interaction between the availability of vitamin $D$ at tissue level (target and secondary lymphoid organs) and the extent to which an immune response is initiated or thriven, may reveal underlying mechanism for associations between vitamin D status and MS.

The aim of this chapter is not to give an extensive overview of the current knowledge about either the immune dysregulation in MS, or the association between vitamin D and MS, or the role of vitamin $D$ in the immune response at a cellular level. Since these have been reviewed extensively by us and others, we will refer to these reviews were appropriate. In the present chapter, we will focus on studies exploring the in vivo relevance of the interaction between vitamin $D$ and the immune response. To achieve this, we will discuss studies on correlations between vitamin $\mathrm{D}$ status and relevant immune outcome parameters, and studies on immunological outcome measures in vitamin D supplementation trials, both in healthy controls as well as in patients with auto-immune disease including MS. We will integrate these findings and discuss considerations for the design of trials on the immune modulating effects of vitamin D in MS.

\section{Multiple sclerosis}

Multiple sclerosis is an inflammatory, demyelinating disease of the central nervous system (CNS). The prevalence is $30-100$ per 100,000 individuals. [106, 107] It is twice as common in females as in males, with an incidence between 20-50 years, and a peak incidence at 30 years of age. $[19,108]$ MS patients have a heterogeneous disease presentation. In relapsing remitting MS (RRMS), periods without neurological exacerbations are altered by periods of increased neurological symptoms. These relapses can be characterised by symptoms of loss of motor and/or sensory function, lack of coordination, but also mental and physical fatigue and/ or cognitive decline. After several years, $65 \%$ of the RRMS patients becomes secondary 
progressive (SPMS) and they experience constant neurological deterioration. A small fraction of the patients experience a progressive accumulation of disability from the beginning of the disease onwards, called primary progressive MS (PPMS). [19, 108]

The exact aetiology of MS is still unknown, but one of the main hypotheses is that it is a Tcell mediated auto-immune disease. This model for MS has been reviewed by several authors. [106, 109] Shortly, it is thought that auto-reactive lymphocytes are primed with an unknown auto-antigen in the secondary lymphoid organs by antigen presenting cells (APC). Clonally expanded and activated effector $T$ cells migrate through the blood brain barrier (BBB) into the perivascular space. Here, the T cells are reactivated by APCs, like perivascular macrophages or dendritic cells (DC), and migrate into the brain parenchyma. In the parenchyma, the T cells contribute to an inflammatory response in which loss of oligodendrocytes and myelin occurs, often followed by loss of neurons. It is a matter of debate whether MS is truly a classical auto-immune disease, with an auto-reactive response directed against a single auto-antigen. [109] Several lines of evidence suggest a general lack of control of the T cell response in MS. Firstly, many genetic polymorphisms associated with MS are involved in the maintenance of T cell homeostasis. [110] Secondly, several differences in general characteristics of the circulating T cell compartment have been found between MS and control patients. During active disease, increased proportions of pro-inflammatory T helper type 1 (Th1) and Th17 cells, as well as increased transcription of IL-17 mRNA have been described. [111-114] Most notably, the capacity of regulatory T cells (Treg) to suppress polyclonal or antigen-specific proliferation of effector T cells was reduced in MS. [115-117] In addition, a reduced transcription of Treg-related genes by peripheral blood mononuclear cells (PBMC) $[118,119]$, and a reduction of Treg-subsets with specific surface molecules as CD39 [120] have been described. Interestingly, these $\mathrm{CD} 9^{+}$Tregs are able to suppress IL-17 production by activated T cells. [121] Accordingly, our group found a negative correlation between the proportion of $\mathrm{CD}_{3} 9^{+}$Tregs and the proportion of Th17 cells in the circulation of RRMS patients. [122] Thirdly, the CNS tissue of MS patients is infiltrated with $\mathrm{CD}^{+}$but predominantly $\mathrm{CD}^{+} \mathrm{T}$ cells. [123] The clonality of these cells is diverse, and does not differ between lesions and normal appearing white matter (NAWM). [124] Beside T cells, B cells are also found in increased number in the CNS of MS patients. [123] Interestingly, oligoclonal intrathecal IgGproduction against predominantly several myelin proteins and heat shock proteins is a hallmark of the disease process. [125] Lastly, currently registered MS disease modulating therapies interfere with either phenotypic and functional characteristics of circulating T cells [126, 127], migration of lymphocytes into the CNS [128], or deplete B and T cells from the circulation $[129,130]$.

\section{Multiple sclerosis and vitamin D}

Several epidemiologic and genetic studies show that a poor exposure to vitamin D is a putative risk factor for developing MS, as has been reviewed elsewhere. $[70,131]$ In brief, genetic 
polymorphisms within key enzymes involved in vitamin D metabolism, as well as within the vitamin D receptor (VDR), have been associated with the risk on developing MS [110, 132], as well as a poor vitamin D status prior to disease onset [133]. In established MS, a poor vitamin D status is associated with a high risk of relapses $[71,74,103]$ and a high MS-related disability score $[73,103]$. Supplementation of high doses of vitamin $D_{3}$ in MS patients did not provoke hypercalcaemia and was well tolerated in several pilot studies. [75, 78, 79] A pilot study in MS on treatment with the biologically active metabolite of vitamin $\mathrm{D}, 1,25(\mathrm{OH})_{2} \mathrm{D}_{3}$, reported the development of a hypercalcaemia in 2/15 subjects. [134] Exploratory analyses on clinical endpoints suggested a reduction of magnetic resonance imaging (MRI) activity in an uncontrolled study [78], a reduction of relapse activity in an uncontrolled [79] and controlled study [75], and a reduction of disability progression in a controlled study design [75]. A study supplementing vitamin $D_{2}$, did not show clear difference on primary MRI endpoints between the high- and low-dose treatment arms. [81] Subsequently, first clinical trials are currently being performed to assess the efficacy of high dose vitamin $D_{3}$ supplementation in MS. [135, 136] Although vitamin D appears to be implicated in the disease process of MS, the underlying mechanism is not known.

\section{Vitamin D metabolism}

Vitamin D is the precursor of an essential hormone. [137] Its natural sources are diet, both from vegetable origin (ergocalciferol or vitamin $D_{2}$ ) as well as from animal origin, especially fatty fish (cholecalciferol or vitamin $D_{3}$ ), and most importantly synthesis in the skin under the influence of ultraviolet B radiation (UVB) in sunlight (90-100\%). The epidermal layer of the skin contains 7-dehydrocholesterol which is transformed into pre-vitamin $D_{3}$ under influence of UVB radiation and body temperature. Subsequently, most of the vitamin D metabolites in the circulation are of vitamin $D_{3}$ origin and only a negligible part of vitamin $D_{2}$. In the circulation and tissues, vitamin $D$ is mostly present as 25 -hydroxyvitamin $D(25(O H) D)$, which is formed in the liver. The circulating levels of this metabolite are most useful to describe an individual's vitamin D status, although $25(\mathrm{OH}) \mathrm{D}$ is not biologically active. A second hydroxylation step is needed to obtain the biologically active metabolite of vitamin $D, 1,25$ dihydroxyvitamin $\mathrm{D}\left(1,25(\mathrm{OH})_{2} \mathrm{D}\right)$. This step is catalysed by the enzyme cytochrome P27B1 (CYP27B1 or 25(OH)D-1 $\alpha$ hydroxylase). [138] $1,25(\mathrm{OH})_{2} \mathrm{D}$ in the circulation is almost exclusively produced by the kidney [139], and circulating levels are regulated by the endocrine control of calcium homeostasis [137]. However, many other tissues can 'activate' 25(OH)D. [140] Once activated, $1,25(\mathrm{OH})_{2} \mathrm{D}$ behaves as a steroid hormone. It binds to the intracellular VDR which regulates the transcription of more than 500 genes containing the vitamin $D$ response elements (VDRE). Catabolism of $1,25(\mathrm{OH})_{2} \mathrm{D}$ is regulated by the enzyme CYP24A1 $\left(1,25(\mathrm{OH})_{2} \mathrm{D}\right.$ 24-hydroxylase). [141] Several cut-off points for an optimal vitamin D status have been proposed, mostly based on its role in the endocrine control of calcium homeostasis. [141-145] Since the relevance of these cut-off values for other biological functions of vitamin $D$ are uncertain, they are not very useful in research. 


\section{Vitamin D as an immune modulator}

All immune cells involved in the adaptive immune response have the potential to metabolize and to respond to vitamin D, as has been reviewed extensively. $[105,146,147]$ Shortly, resting and activated myeloid cells, including monocytes, macrophages and DCs, and activated T- and B-lymphocytes express the VDR. [148-151] In lymphocytes, exposure to $1,25(\mathrm{OH})_{2} \mathrm{D}$ further enhances VDR expression. $[148,151]$ Myeloid APCs, and most notably the specialized $\mathrm{DCs}$, lose their antigen presenting potential upon exposure to $1,25(\mathrm{OH})_{2} \mathrm{D}$, and skew towards an immature innate profile. [152] Subsequently, they have less capability to start a $\mathrm{T}$ cell response. [153] Accordingly, 1,25(OH) $)_{2} \mathrm{D}$ inhibits $\mathrm{T}$ cell proliferation and the production of pro-inflammatory cytokines as IFN- $\gamma$ and IL-17. [154] The synthesis of anti-inflammatory cytokines, such as IL-4 and IL-10, is promoted. Besides being targets of vitamin D, activated myeloid cells (either activated by adaptive or innate stimuli) and lymphocytes (either activated polyclonally or via antigen specific stimulation) express the activating enzyme CYP27B1, and subsequently synthesize $1,25(\mathrm{OH})_{2} \mathrm{D}$ locally. [149, 154-158] All these cells also upregulate the catabolic enzyme CYP24A1 upon exposure to $1,25(\mathrm{OH})_{2} \mathrm{D}$. $[149,154,156]$ Since these observations are done in vitro, it is uncertain whether these dynamics also apply to immune activation within tissues where the immune response becomes manifest, i.e. the secondary lymphoid tissues as well as the peripheral tissues affected by a disease. Nevertheless, studies in the experimental MS model auto-immune encephalomyelitis (EAE) showed an up-regulation of VDR and CYP27B1 and a down-regulation of CYP24A1, together with an enhanced local $1,25(\mathrm{OH})_{2} \mathrm{D}$ production within the inflamed CNS. [159] Accordingly, supplementation of vitamin $\mathrm{D}$ or treatment with $1,25(\mathrm{OH})_{2} \mathrm{D}$ prevented development of EAE and ameliorated the disease course. $[160,161]$ Both in the circulation, lymph nodes, and CNS, proliferation and cytokine assays suggested a shift of the immune response towards a less inflammatory profile. [131]

\section{Vitamin D status, vitamin D supplementation and the effect on immune regulation in vivo}

Since an auto-reactive T cell response has been implicated in the disease process of MS, an immune modulating, dampening effect of vitamin D may be very relevant. [104] Although effects of vitamin D metabolites on immune cells in vitro have been frequently investigated, in vivo data are limited. The negative correlation between vitamin $\mathrm{D}$ status and the risk on developing MS was observed within the physiologically occurring ranges of vitamin $D$, which is $0-150 \mathrm{nmol} / \mathrm{L}$. [133] Within the same range of $25(\mathrm{OH}) \mathrm{D}$, a negative correlation between vitamin $D$ status and MS relapse rate was found. $[71,74,103]$ If the driving force of these correlations is the promotion of $T$ cell homeostasis within the higher ranges of serum $25(\mathrm{OH}) \mathrm{D}$, relevant measures of immune regulation would be expected to correlate positively with vitamin $\mathrm{D}$ status in vivo. 
We conducted a literature search to identify papers which investigated either the correlation of serum $25(\mathrm{OH}) \mathrm{D}$ levels with measures of immune homeostasis, or the effect of vitamin $\mathrm{D}$ supplementation on immune parameters determined in peripheral blood (correlation studies are summarized in Table 1, supplementation studies in Table 2). We focused on studies in healthy controls, patients with MS, and patients with other auto-immune diseases. Since the systemic metabolism of vitamin D is disturbed in kidney disease patients, we excluded studies done in this patient population. Furthermore, studies in cancer patients, HIV patients, allergic patients and patients with an infectious disease were excluded, because here the interaction of vitamin $D$ with the immune system may be different from auto-immune diseases due to distinct immunopathology. Moreover only studies in which $25(\mathrm{OH}) \mathrm{D}$ levels were measured and reported were included. Epidemiological studies addressed association between serum $25(\mathrm{OH}) \mathrm{D}$ levels, and the efficacy of vitamin $\mathrm{D}_{2} / \mathrm{D}_{3}$ supplementation (vide supra). Additionally, while the effect of vitamin $D$ on the immune system is most likely mainly executed by local activation of vitamin $D$, this is rather dependent on circulating $25(\mathrm{OH}) \mathrm{D}$ than on $1,25(\mathrm{OH})_{2} \mathrm{D}$ levels. Therefore only supplementation studies in which vitamin $\mathrm{D}_{2}$ or $\mathrm{D}_{3}$ was supplemented and in which the effects of vitamin D supplementation could be distinguished from other possible co-interventions, were included. Since several approaches have been used to evaluate the effect of vitamin D on the immune function in vivo, we will discuss the different immunological outcome measures that have been used in these studies. 


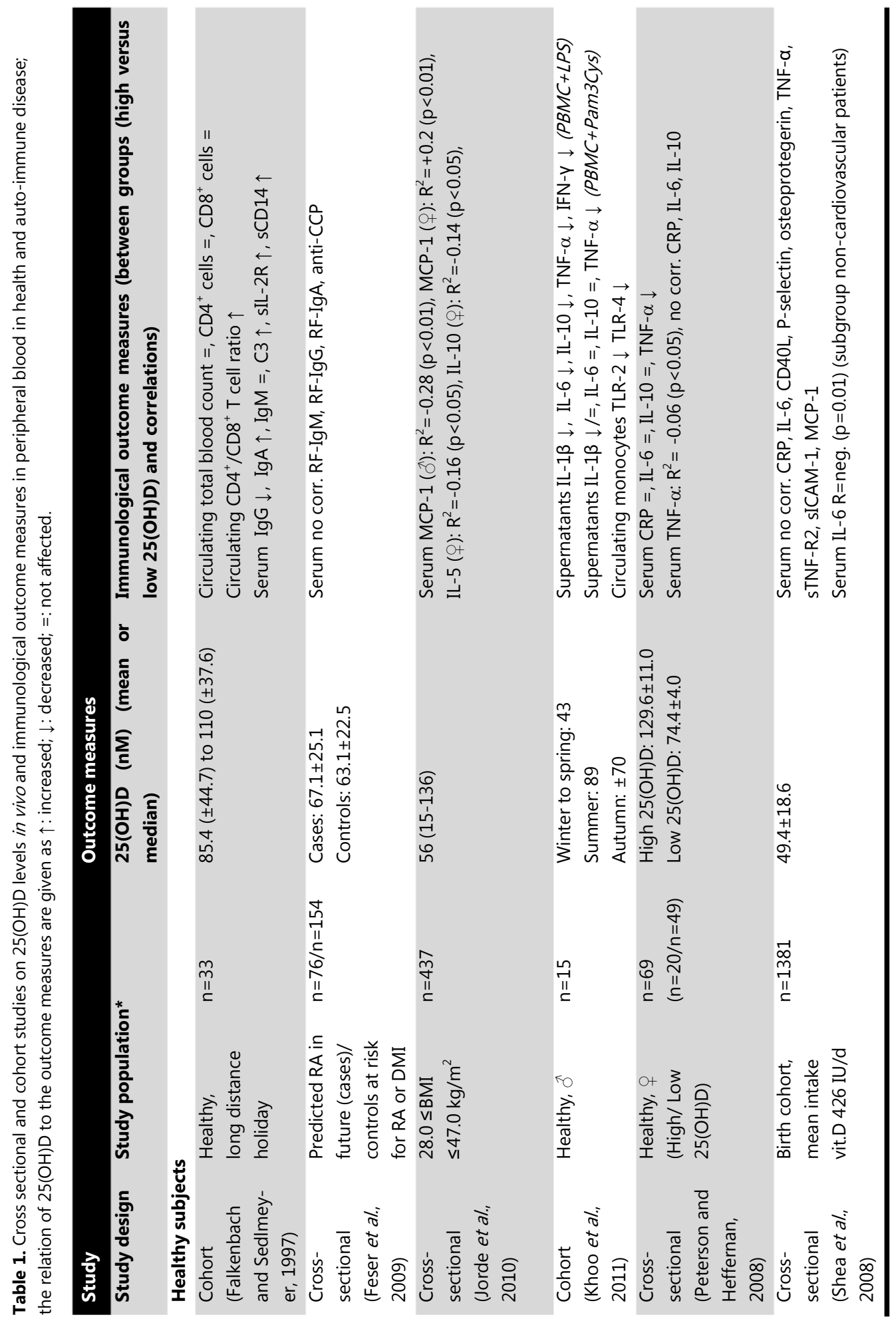




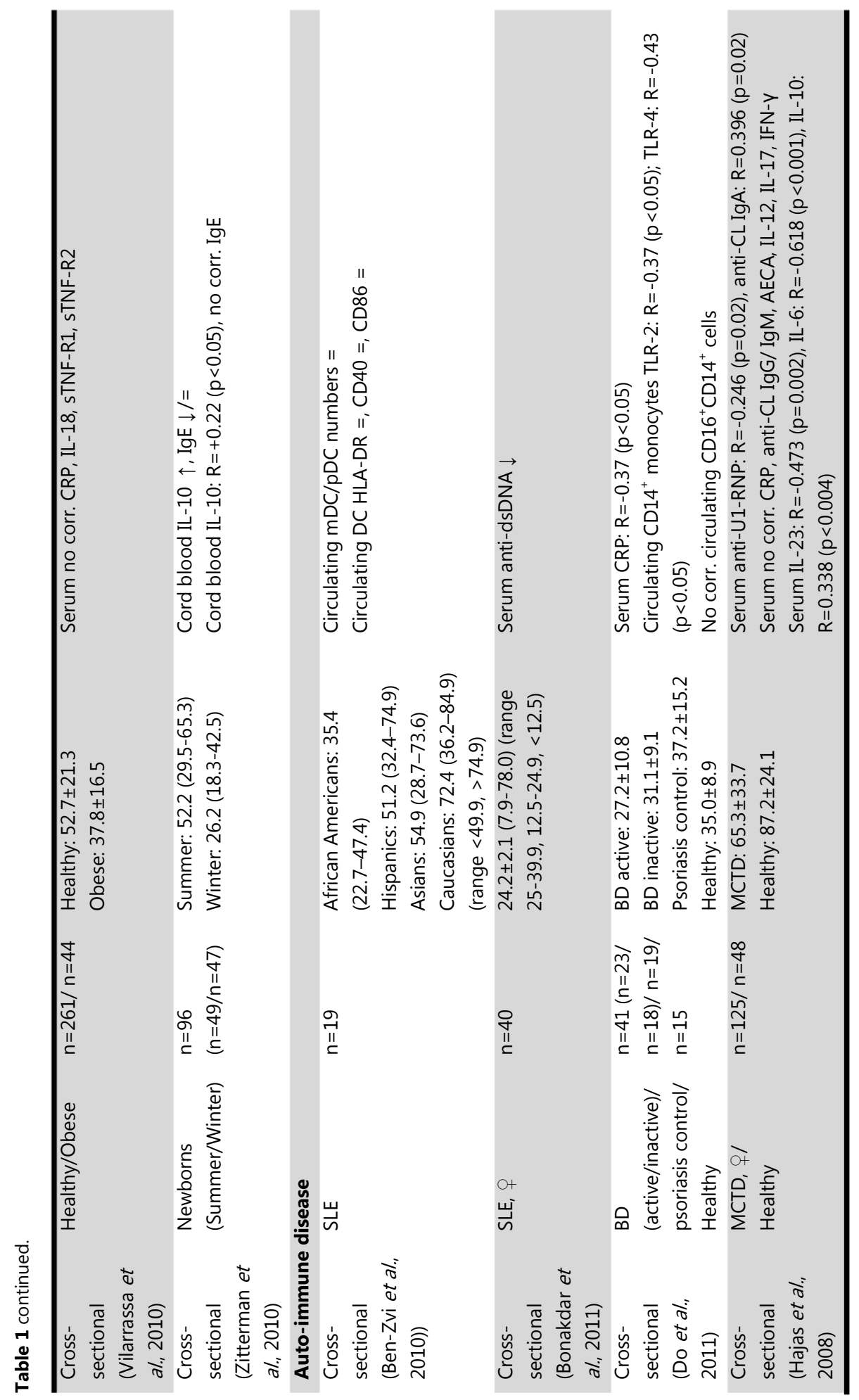




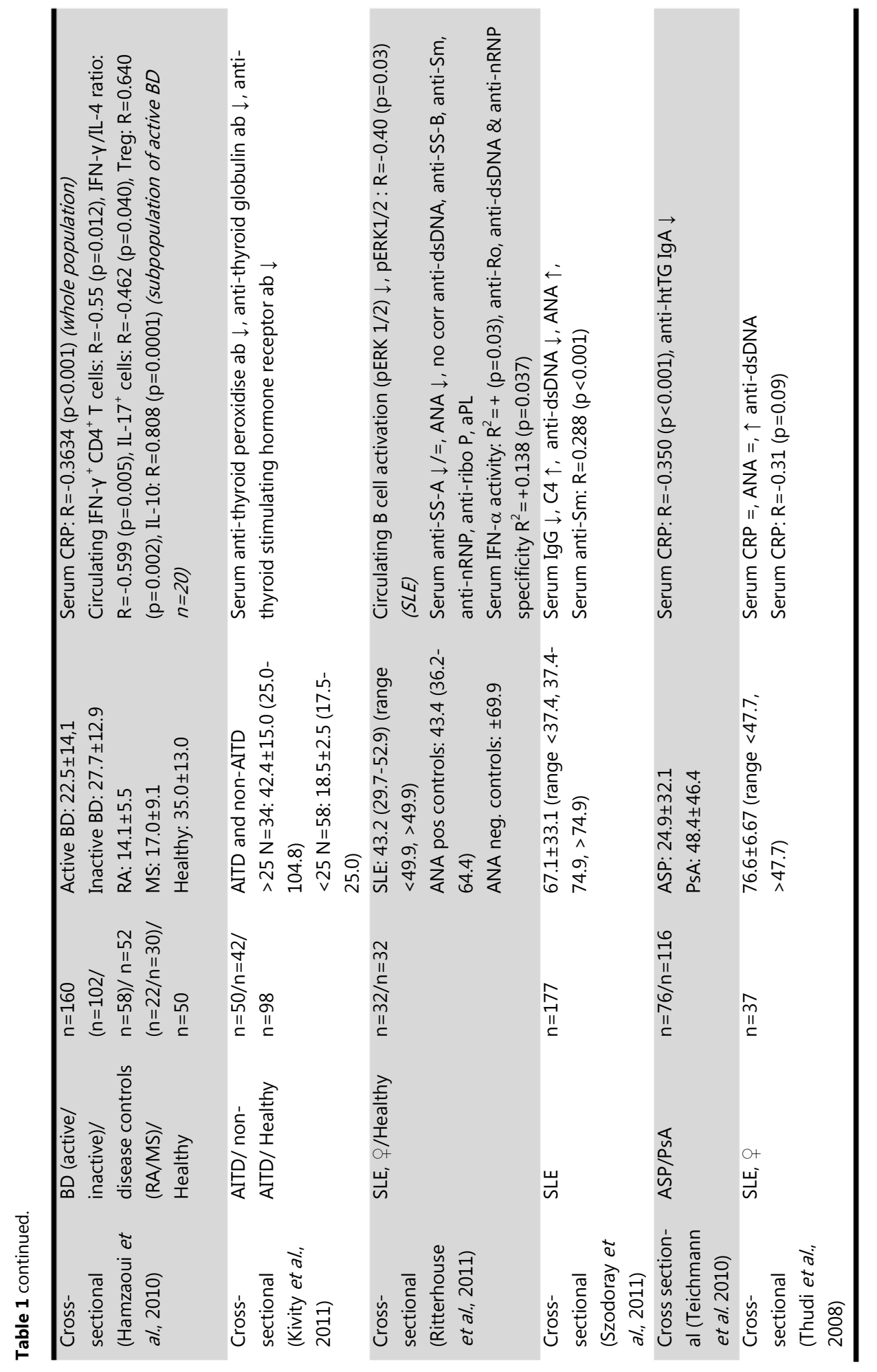




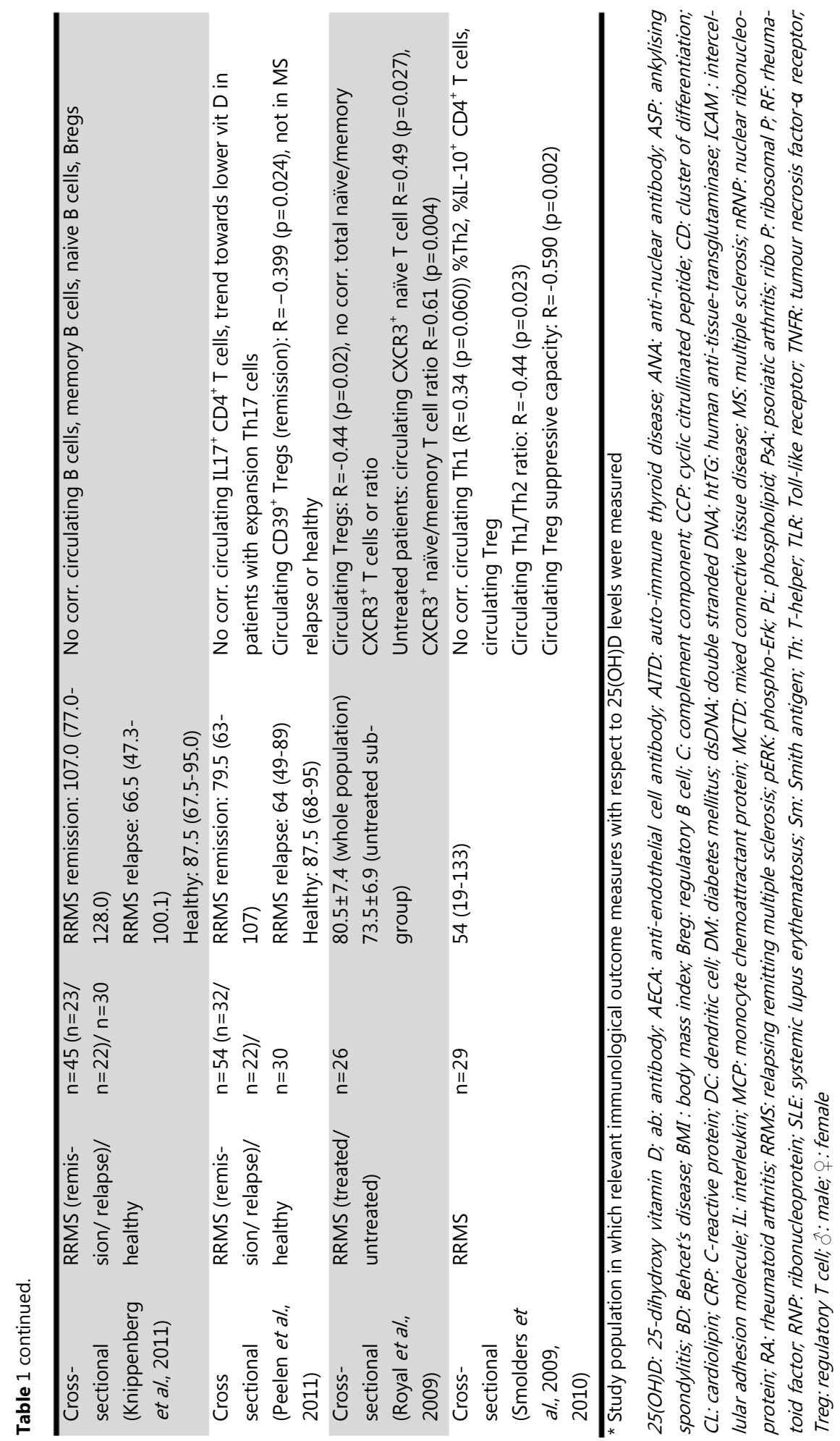




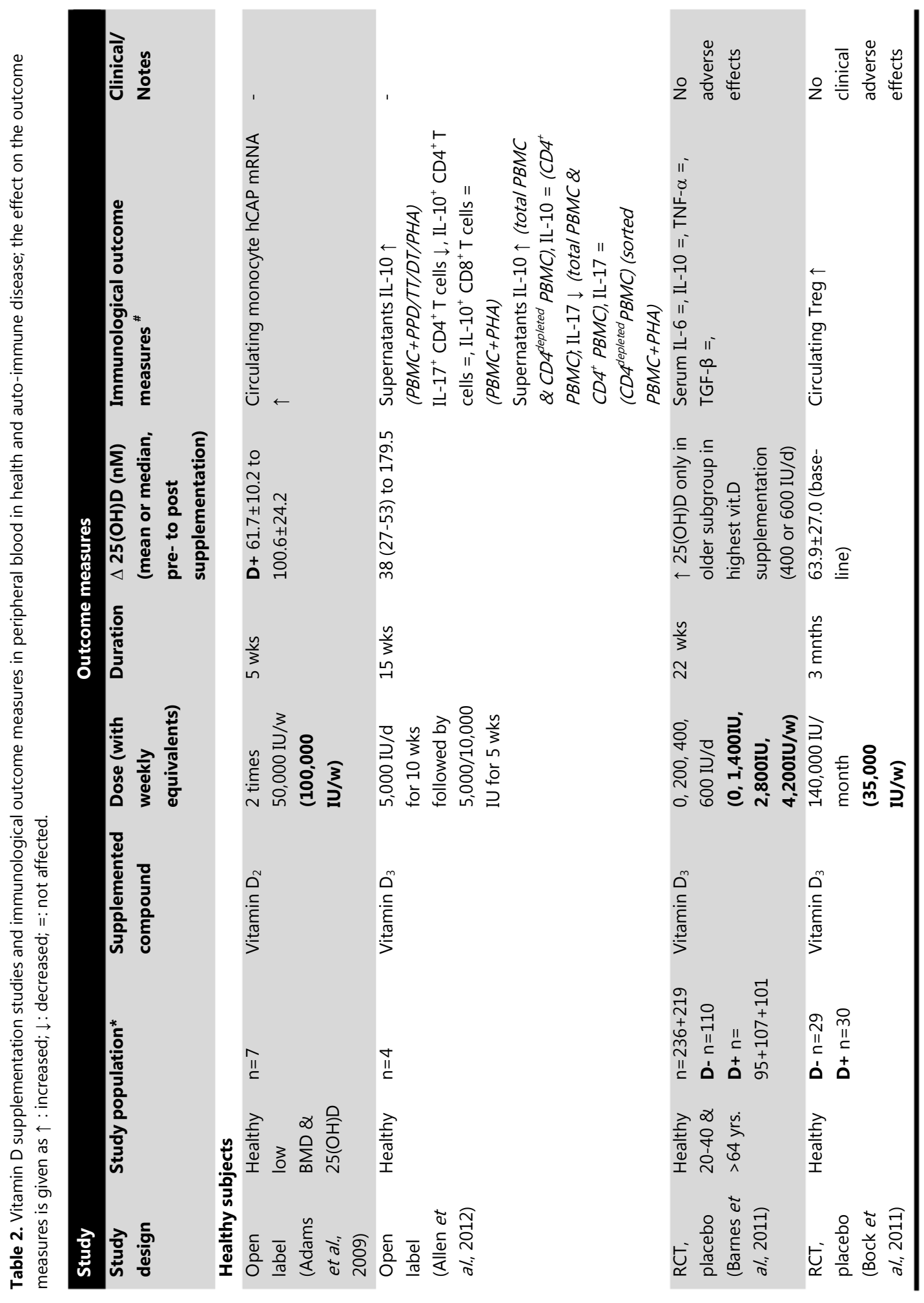




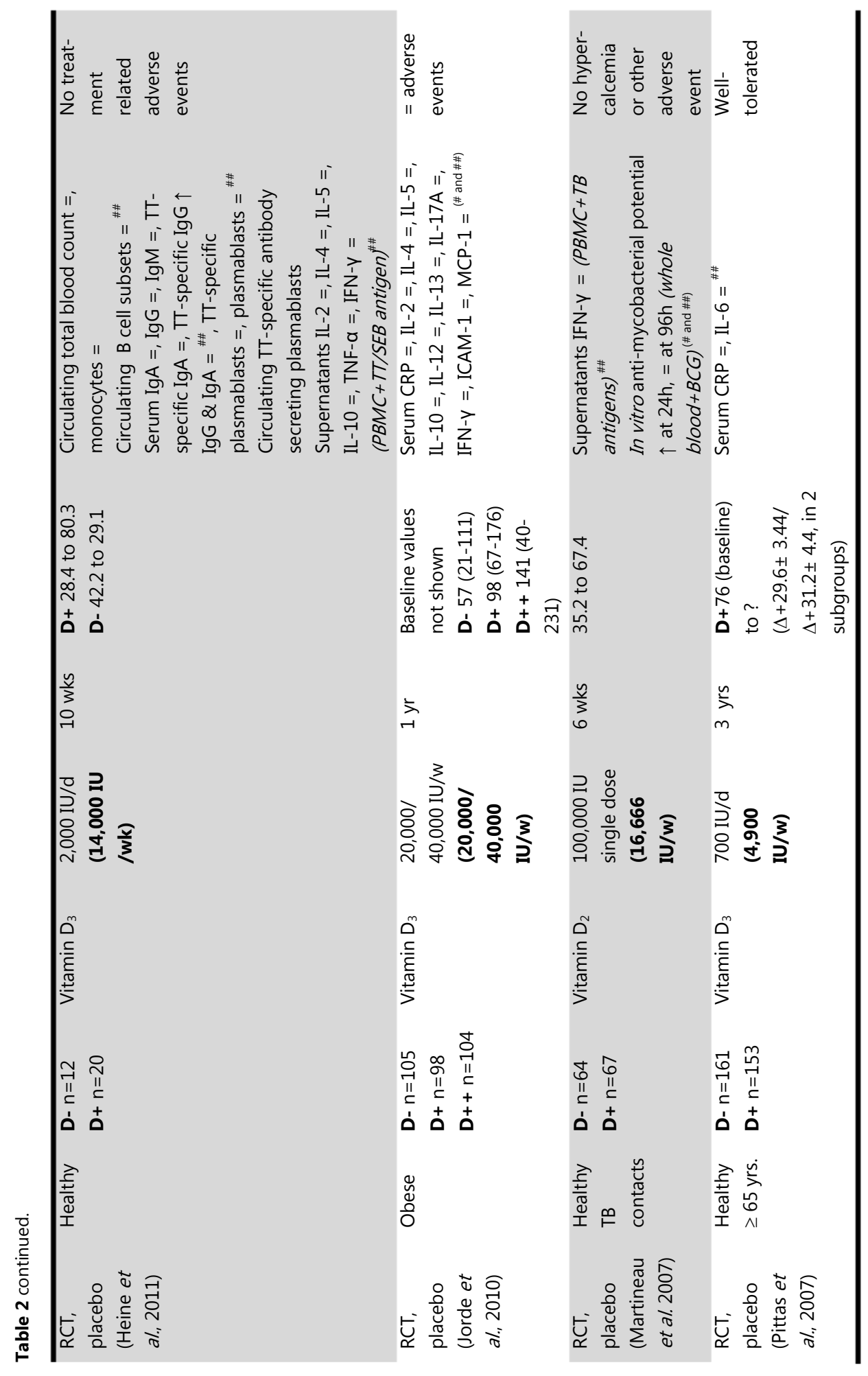




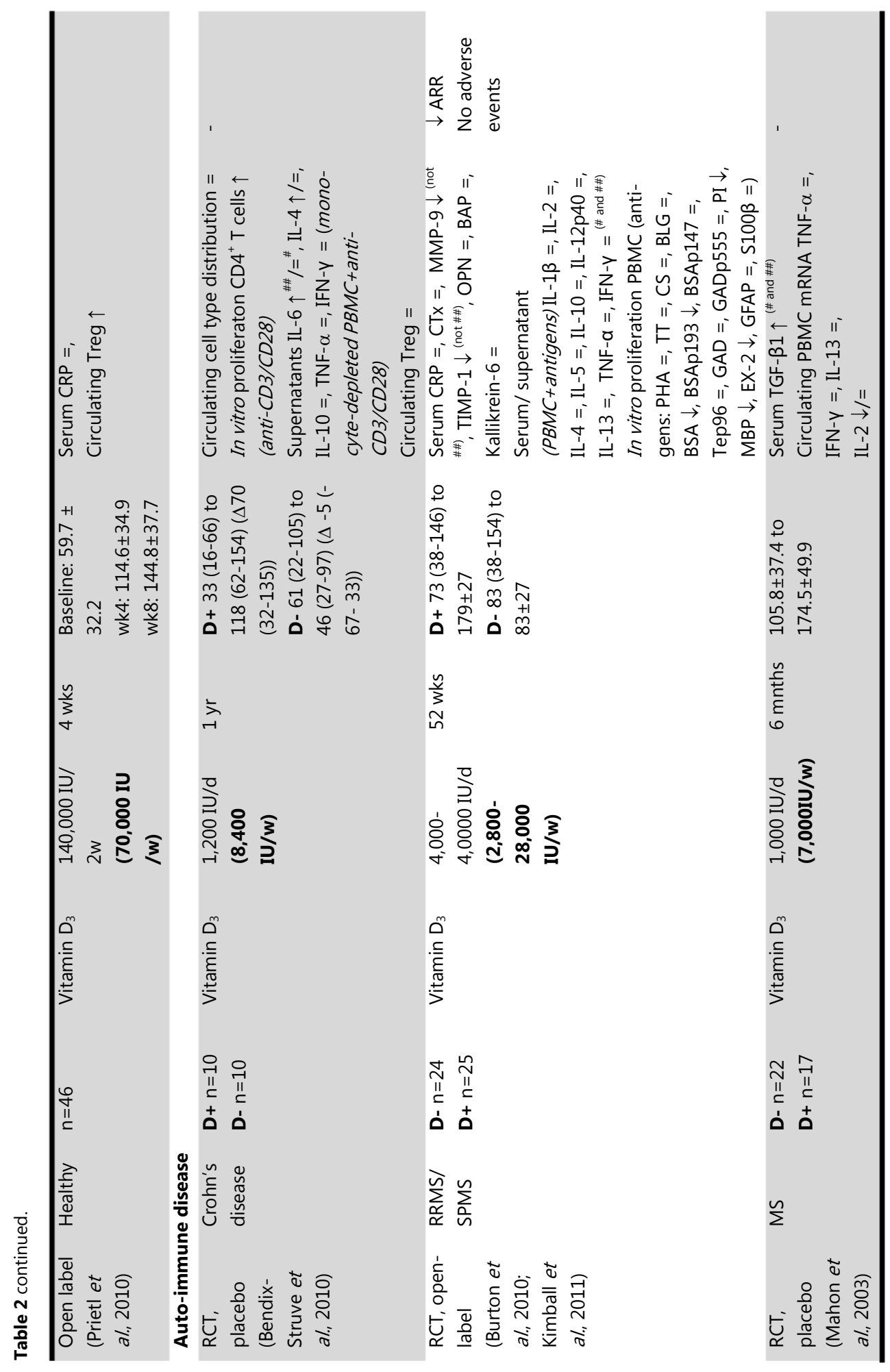




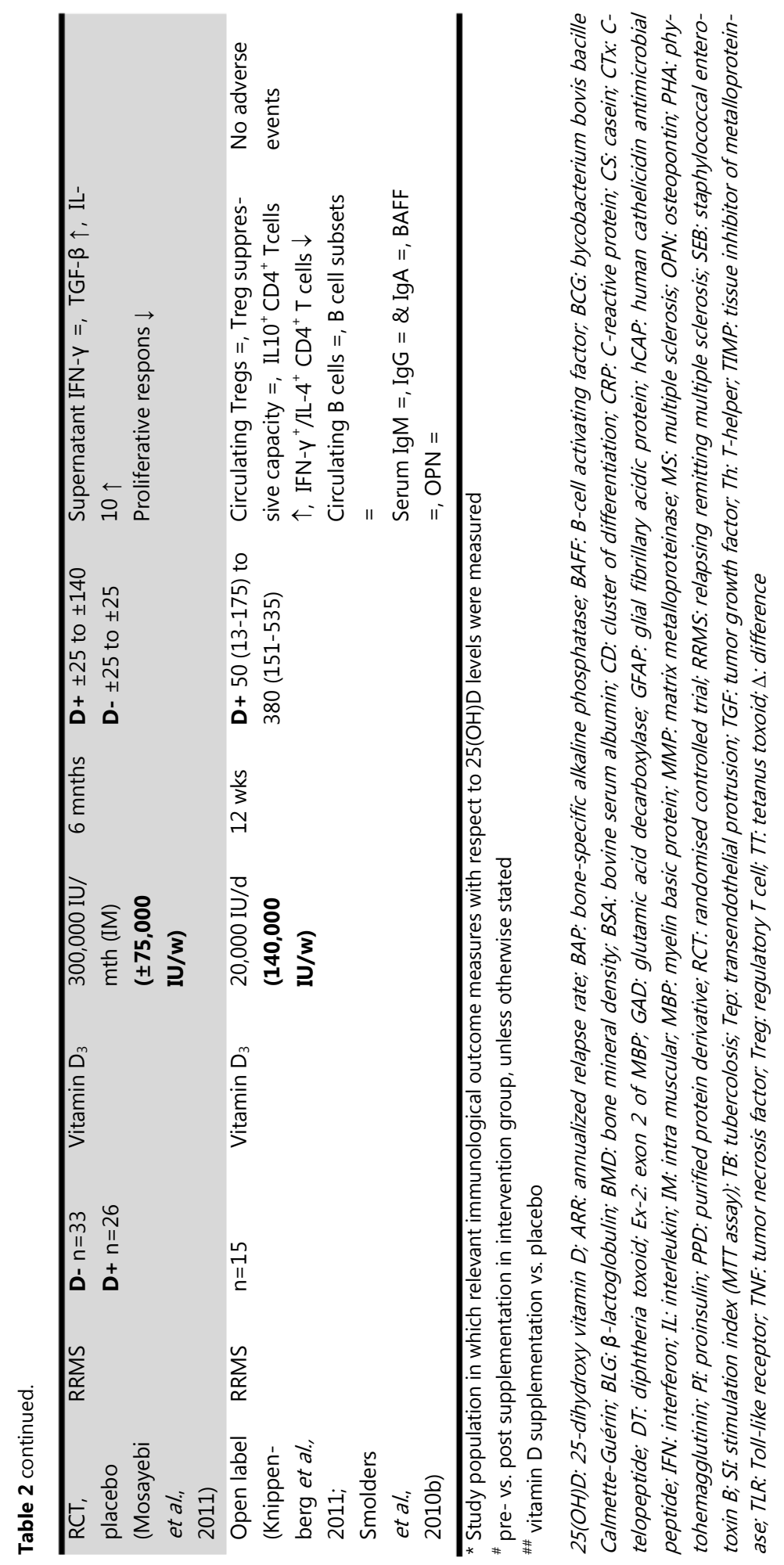




\section{Inflammatory markers in the circulation}

First of all, several groups addressed correlations of serum 25(OH)D with serum concentrations of general markers of inflammation, chemokines, and cytokines. Serum concentrations of C-reactive protein (CRP) did not correlate with serum 25(OH)D in most studies with controls. [162-164] Supplementation of vitamin D did also not influence CRP levels in healthy, older or obese persons [165-167] or in patients with MS [168]. However, a negative correlation between these parameters has been described in patients with systemic lupus erythematosus (SLE) [169], ankylosing spondylitis (ASP) or psoriatic arthritis (PsA) [170] and Behcet's disease (BD) $[171,172]$. Regarding complement, a study among healthy individuals reported higher serum concentrations of both $25(\mathrm{OH}) \mathrm{D}$ and $\mathrm{C} 3$ after a long-distance holiday [173], and higher serum 25(OH)D levels correlated with higher serum C4 in subjects with SLE [174].

\section{Cytokines and chemokines in the circulation}

In healthy controls, multiple studies reported no association of higher serum $25(\mathrm{OH}) \mathrm{D}$ levels with serum concentrations of pro-inflammatory cytokines as IL-6 [162, 163], IL-12 [175], IL-17 [175], IL-18 [164], IFN- $\gamma$ [175], TNF- $\alpha$ [163] and the anti-inflammatory cytokine IL-10 [162]. Accordingly, no correlations were reported with the immune related molecules soluble (s)CD40L [163], sP-selectin [163], osteoprotegerin [163], sTNF-R1 [164], sTNF-R2 [163, 164], sICAM-1 [163] and MCP-1 [163]. Likewise, supplementation studies with vitamin D in healthy controls and MS patients showed no significant differences in serum IL-1 [168], IL-2 [165, 168], IL-4 [165, 168], IL-5 [165, 168], IL-6 [166, 176], IL-10 [165, 168, 176], IL-12 [165, 168], IL13 [165, 168], IL-17 [165], IFN- $\gamma$ [165, 168], TGF- $\beta$ [176], TNF- $\alpha$ [168, 176], sICAM-1 [165], MCP-1 [165], C-telopeptide (CTx) [168], bone specific alkaline phosphatase (BAP) [168], kallikrein 6 [168], osteopontin (OPN) [168, 177] or B-cell activating factor (BAFF) [178]. Despite these negative findings, some studies reported correlations between vitamin $\mathrm{D}$ status and soluble molecules in serum. Higher serum levels of $25(\mathrm{OH}) \mathrm{D}$ have been reported to coincide with lower serum concentrations of TNF- $\alpha$ in healthy females. [162] In cord blood of new-borns, serum 25(OH)D levels correlated positively with levels of IL-10. [179] This correlation was also observed in females with mixed connective tissue disease (MCTD) [175] and active BD patients [172]. Supplementation of vitamin D in patients with MS has been reported to induce increased serum levels of TGF- $\beta$. [180] Conversely, in otherwise healthy obese females, a negative correlation of serum $25(\mathrm{OH}) \mathrm{D}$ with serum IL-5 and IL-10 was reported. [165] A negative correlation with IL-6 and IL-23 has been reported in females with MCTD and healthy controls. $[163,175]$ A long distance holiday induced both higher serum levels of 25(OH)D, sCD14, and sIL-2R. [173] MCP-1 was positively correlated to 25(OH)D levels in obese females, but not in males. [165] Lastly, in MS patients, lower circulating concentrations of tissue inhibitor of metalloproteinase (TIMP)-1 and matrix metalloproteinase (MMP)-9 were reported after supplementation of vitamin D. [168] 


\section{Cytokine excretion by PBMC in supernatants}

Of course, the cellular sources of cytokines detected in serum are uncertain. To identify cells within the PBMC fraction producing these cytokines, several groups analysed the transcription profile of cytokines in PBMC directly ex vivo. Supplementation of vitamin D in healthy subjects increased expression of cathelicidin mRNA in monocytes [181], but did not differentially affect expression of TNF- $\alpha$, IFN- - , IL-2, or IL-13 mRNA in patients with MS [180].

Additionally, several studies measured cytokine excretion in supernatants of specific cell cultures. Most studies cultured PBMC stimulated either with TLR-ligands [182] or with polyclonal [183] or antigen specific T cell stimuli $[168,184,185]$. A higher vitamin D status correlated with a reduced IL- $1 \beta$ and TNF- $\alpha$ production upon TLR- 2 and TLR-4 triggering, while a loss of IL-6, IL-10 and IFN- $\gamma$ was only found with TLR-4 triggering. [182] Upon T-cell stimuli, supernatant levels of IL-1 $\beta$ [168], IL-2 [168, 184], IL-4 [168, 184], IL-5 [168, 184], IL-10 [168, 183, 184], IL-12 [168], IL-13 [168], IFN- $\nu$ [168, 183-186], and TNF- $\alpha$ [168, 183, 184], were not affected by supplementation of vitamin D, while TGF- $\beta$ [186] and IL-10 increased [186, 187] and IL-17 decreased [187]. The positive effect on IL-10 was mainly due to an increased production of IL-10 by the CD4 ${ }^{+}$depleted PBMCs, while this cell population did not produce IL17 [187]. A study on polyclonal stimulation of monocyte-depleted PBMC of patients with Crohn's disease reported a trend towards an increased concentration of IL-4 and IL- 6 in the supernatants after supplementation. [183]

\section{Proliferation of $\mathbf{T}$ cells in vitro}

Another way to measure $T$ cell responses to antigens is to measure induction of proliferation in vitro. A study in Crohn's disease patients reported an increase of polyclonal T cell proliferation after supplementation of vitamin D [183], while in MS patients proliferation was decreased [186] or unaffected [79]. Kimball et al. tested proliferative responses of $\mathrm{T}$ cells against a large panel of antigens in MS patients. [168] After supplementation of vitamin $D$, proliferative responses against BSA, BSAp193, PI, MBP and exon 2 of MBP (EX-2) were reduced compared with prior to supplementation.

\section{Phenotyping PBMC directly ex vivo}

Several studies analysed circulating myeloid and lymphocyte subsets and phenotypes with flow cytometry. Two studies reported a negative correlation between serum 25(OH)D levels and monocyte expression levels of both TLR-2 [171, 182] and TLR-4 [171, 182]. There was neither a correlation with the circulating proportions of myeloid and plasmacytoid DCs, nor with the expression of human leukocyte antigen (HLA)-DR, CD40, or CD86 on these cells. [188] There was also no correlation with the proportion of $\mathrm{CD} 14^{+} \mathrm{CD} 16^{+}$monocytes. [171] Vitamin $D$ supplementation did not affect the total proportion of circulating monocytes. [184]

Regarding lymphocytes, both $B$ and $T$ cell subsets have been characterized. A correlation was described of $25(\mathrm{OH}) \mathrm{D}$ levels with the percentages of total naïve $\left(\mathrm{CXCR} 3^{+}\right)$and total memory 
$\left(\mathrm{CXCR}^{+}\right) \mathrm{CD}^{+} \mathrm{T}$ cells in an MS cohort not treated with immune modulating therapies [189]. In patients with $\mathrm{BD}$, a positive correlation between $25(\mathrm{OH}) \mathrm{D}$ and total proportions of Tregs was reported. [172] Such a correlation was not confirmed in patients with MS [190], and was even contradicted by a negative correlation in another MS population. [189] When assessing subsets of Tregs, lower circulating proportions of $\mathrm{CD} 39^{+}$Tregs correlated with higher serum 25(OH)D levels in MS patients during disease remission. [122] Additionally, the capacity of Tregs to control polyclonal T cell proliferation was also improved in MS patients with higher serum 25(OH)D levels. [191] Supplementation of vitamin D has been reported to induce higher circulating proportions of Tregs in healthy individuals. [167, 192] Contrastingly, supplementation of vitamin $D$ did not result in higher proportions of naïve/memory Tregs in subjects with MS and Crohn's disease [79, 183], and Treg suppressive capacity was also not significantly affected [79]. Regarding $T$ helper cell subsets, a study in BD patients reported negative correlations between serum $25(\mathrm{OH}) \mathrm{D}$ and the circulating proportion of Th1 and Th17 cells, and with the Th1/Th2-ratio. [172] Although a study in MS patients could not detect correlations with the proportions of T helper cell subsets, the Th1/Th2 ratio correlated also negatively with serum 25(OH)D. [191] In a cross-sectional study among MS patients, serum 25(OH)D levels tended to be lower in patients with an expanded Th17 compartment. [122] Supplementation of vitamin D in healthy subjects resulted in a decline of Th17 cells, while the number of IL-10+ $\mathrm{T}$ cells did not change. [187] Supplementation of vitamin D in subjects with MS resulted in a decreased Th1/Th2-ratio, as well as an expanded proportion of IL-10 ${ }^{+}$T cells. [79]

With respect to $B$ cells, a study in SLE reported decreased $B$ cell activation in subjects with high 25(OH)D levels. [193] However, in subjects with MS, no correlation of serum 25(OH)D with circulating $B$ cell subsets, including regulatory B cells (Breg), could be found. [178] Accordingly, supplementation of vitamin D did not affect B cell subset distribution in both subjects with MS and healthy control donors. [178, 184] B cells are precursors to Igproducing plasma cells. A study in SLE and in healthy individuals on long distance holiday reported lower total IgG concentrations in serum with increasing 25(OH)D level $[173,174]$, which might be in line with the reduced $B$ cell activation observed in the cross-sectional study [193]. IgE production seems to be affected negatively by 25(OH)D [179], while IgA production was increased and IgM production was not correlated [173]. On the other hand, other studies found no effect of vitamin $D$ supplementation on total serum polyclonal IgG, IgM and IgA in healthy subjects and subjects with MS. [178, 184] However, antigen specific antibody-production may be more relevant than the polyclonal compartment. Indeed, supplementation of vitamin D improved tetanus toxoid (TT)-specific IgG-response in TT vaccination. [184] In several SLE cohorts contrasting results have been found regarding antinuclear antibody (ANA) and anti-double stranded (ds)DNA antibody production. [169, 174, 193, 194] A negative correlation for serum $25(\mathrm{OH}) \mathrm{D}$ with anti-thyroid antibodies in thyroid disease patients [195], with serum anti-human tissue transglutaminase (htTG) IgA in ASP and PsA patients [170] and a trend towards such a correlation with anti-SS-A antibodies in SLE [193] 
have been reported, while there was a positive correlation with serum anti-Smith antibodies [174]. In donors at risk for rheumatoid arthritis (RA), there was no correlation of 25(OH)D with either rheumatoid factor (RF) or anti-cyclic citrullinated peptide (CCP) antibodies. [196] In patients with MCTD, serum anti-U1-ribonucleoprotein (RNP) antibody correlated negatively and serum anti-cardiolipin $(\mathrm{CL})$ IgA positively with serum $25(\mathrm{OH}) \mathrm{D}$, while no correlation with CL IgG/IgM and anti-endothelial cell antibodies (AECA) was found. [175]

\section{Discussion and conclusion}

Our literature search revealed evidently that studies done so far are heterogeneous in many aspects. Study populations range from healthy controls to at risk persons and patients with distinct auto-immune diseases. Moreover, most studies seem to be underpowered and have a lack of appropriate controls. Beside this, 25(OH)D status is not always properly reported. This is regrettable, since an effect of vitamin $D$ on the immune system would be expected in the highest or higher than physiological ranges of 25(OH)D levels. [197] Without baseline $25(\mathrm{OH}) \mathrm{D}$ levels, it is difficult to discriminate between the effects of vitamin $\mathrm{D}$ in individuals with sufficient versus insufficient vitamin $D$ status. Besides this, $25(\mathrm{OH}) \mathrm{D}$ assays have a great interassay variability [198] and cut-off points used for defining 25(OH)D insufficiency and sufficiency are not standardized. Furthermore, correlation studies have the potential bias of reverse causality: vitamin $D$ status might not be responsible for the possible improvement in clinical and immunological outcomes in disease, but disability itself might influence the vitamin D status of individuals. Supplementation studies are not subjected to reverse causality, however, these also show great diversity in the supplemented compound (either vitamin $D_{2}$ or vitamin $D_{3}$ ) and in the dose and duration of supplementation. The supplemented doses range from doses which do not affect serum 25(OH)D levels significantly to doses which lead to supra-physiological serum $25(\mathrm{OH}) \mathrm{D}$ levels. These factors make the individual studies hardly comparable, and readily explain the many contradictive results. Finally, it is also important to keep a publication bias in consideration. In general, data suggest a trend towards a promotion of anti-inflammatory/regulatory cytokines and cells to be present, but this is certainly not substantiated by conclusive data.

On top of that, analyses of the studies thus far showed a diverse repertoire of immunological outcome measures. These outcome measures form a continuum, ranging from soluble markers of general inflammation which can be measured easily in large cohorts of study subjects, to labour-intensive, detailed assessment of specific cellular processes. We argue that the choice between either of these outcome measures should primarily be driven by the specific disease outcome that is to be studied. As described above, $1,25(\mathrm{OH})_{2} \mathrm{D}$ interferes in vitro with proliferation and cytokine secretion by activated PBMC. These in vitro models reflect most likely the sites in the body where antigen presentation and immune activation take place, which are the secondary lymphoid organs and the tissues targeted by autoimmune diseases. In these tissues, the interaction of vitamin D with the immune response is 
unlikely to depend on either a single cell type or cytokine, but rather on a complex interplay between multiple vitamin $D$ sensitive cells of the innate and adaptive immune system. [147] When analysing correlations between serum 25(OH)D levels and immunological parameters in the blood, one has to realize that there is a mismatch in space and time related to in vitro experiments. Immune cells in the circulation are on the move between target sites and lymphoid tissue, and soluble factors are derived from both specific immune responses of interest as well as from other cells. Most informative would be assessment of cells and cytokines in lymph nodes and target organs, but sampling of material is obviously not feasible in most diseases. Another issue to keep in mind is that effects of vitamin D on immune parameters, as described in this chapter, might be secondary to other effects of vitamin D. For instance, the positive correlation between 25(OH)D and C4 in SLE might be due to an immune independent effect on disease activity; since SLE is an immune complex mediated disease associated with complement activation via the classical pathway, any reduction in disease activity will result in normalisation of C4 levels. Similarly, the negative association of 25(OH)D levels with several auto-antibodies might not be due to a direct effect of vitamin D on B cells, but might be mediated via an effect on auto-antigen presentation by APCs. In addition, many auto-immune diseases are associated with infections [195] causing inflammation and subsequent induction of auto-immune disease manifestations. Since vitamin D also affects the innate immune system via the production of beta-defensins and cathelicidins, this will reduce the infectious load and thereby the inflammatory response and eventually will affect the adaptive immune system. To substantiate an immune modulating effect of vitamin $D$ in vivo, the best approach appears to be assessment of the end products of the immunological cascade in the circulation, which are most relevant to the disease or health outcome studied. For auto-immune diseases, analysis of auto-antibody titres or lymphocyte cell proliferative and cytokine responses against auto-antigens are more logical choices than assessment of polyclonal IgG levels, polyclonal T cell proliferation, or serum cytokine levels. In MS, where no single auto-antigen is present, studies in which immunological outcomes have been associated with disease in patient-control studies, or have been associated with exacerbation activity or remission of disease, seem most relevant. Ultimately, only well-powered, controlled studies in well-defined cohorts measuring disease-specific relevant outcome measures will be most informative on an immune modulating potential of vitamin $D$.

Currently, several clinical trials are investigating the efficacy of vitamin D supplementation in MS and other auto-immune diseases. These trials provide an excellent framework to design potentially conclusive studies on the immune modulating effects of vitamin $D$ in vivo. It would be a missed chance not to address this issue in these studies, especially since insight in the working mechanism of vitamin D may reveal subgroups of patients which may benefit most from vitamin D supplementation. Additionally, a known working mechanism can facilitate hypothesis driven combination with existing treatment regimens, and may reveal a biomarker which allows titration of serum $25(\mathrm{OH}) \mathrm{D}$ levels to an adequate dose. 


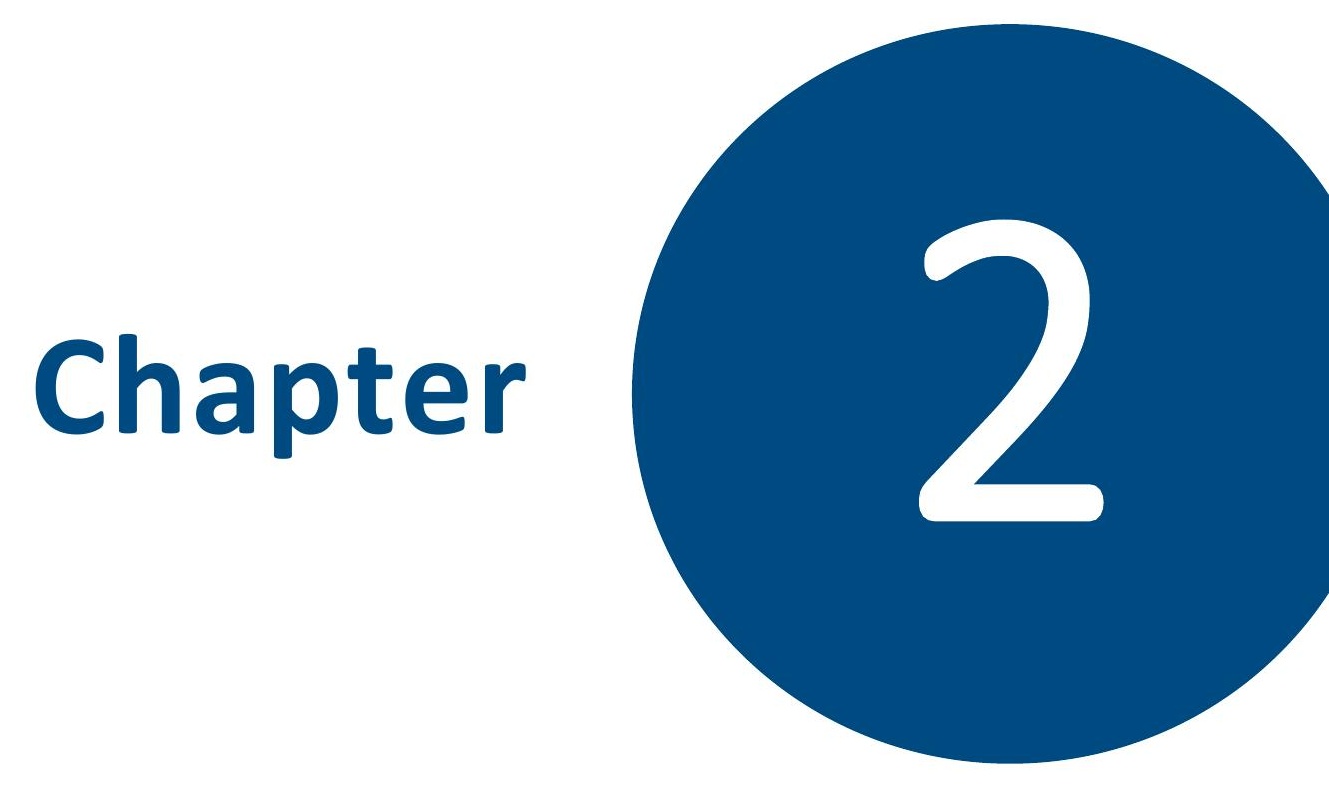

\section{Vitamin D and disability progression in patients with multiple sclerosis}

Published as: Vitamin D status does not affect disability progression of multiple sclerosis patients over three year follow-up

Muris, A.H., Smolders J., Rolf L., Klinkenberg L.J.J., van der Linden N., Meex S., Damoiseaux, J., Hupperts R. PLoS One. 2016 Jun 8;11(6):e0156122. 


\section{Abstract}

The risk of developing multiple sclerosis (MS) as well as MS disease activity is associated with vitamin $D(25(O H) D)$ status. The relationship between the main functional disability hallmark of MS, disability progression, and 25(OH)D status is less well established though, especially not in MS patients with progressive disease.

This retrospective follow-up study included 554 MS patients with a serum baseline 25(OH)D level and expanded disability status scale (EDSS) with a minimum follow-up of three years. Logistic regressions were performed to assess the effect of baseline 25(OH)D status on relapse rate. Repeated measures linear regression analyses were performed to assess the effect on disability and disability progression.

Baseline deseasonalised 25(OH)D status was associated with subsequent relapse risk (yes/no), but only in the younger MS patients ( $\leq 37.5$ years; OR=0.872, per $10 \mathrm{nmol} / \mathrm{L}$ 25(OH)D, $p=0.041)$. Baseline 25(OH)D status was not significantly associated with either disability or disability progression, irrespective of MS phenotype.

Within the physiological range, 25(OH)D status is just significantly associated with the occurrence of relapses in younger MS patients, but is not associated with disability or disability progression over three years follow-up. Whether high dose supplementation to supra physiological 25(OH)D levels prevents disability progression in MS should become clear from long term follow-up of supplementation studies. 


\section{Introduction}

Multiple sclerosis (MS), a demyelinating disease of the central nervous system (CNS), is considered to be an inflammatory disease of auto-immune origin. [19] A low vitamin D status, measured as $25(\mathrm{OH}) \mathrm{D}$, has been associated with an increased risk of developing MS $[70,131]$ and vitamin D related genes, such as CYP24A1 and CYP27B1 emerge from gene wide association studies. [14]

Vitamin D status has also been associated with disease activity i.e. relapse rate. Firstly, MS patients showed lower vitamin D status during relapse than during remission. [154, 199, 200] Secondly, in early relapsing remitting MS (RRMS), higher 25(OH)D levels were associated with an increased chance of being relapse free 24 months prior to serum sampling [103], and with a decrease in the relapse rate in subsequent months by $9-34 \%$ with each $10 \mathrm{nmol} / \mathrm{L}$ increase in $25(\mathrm{OH}) \mathrm{D}$ level. $[71,72,74]$ Some, but not all, small high dose supplementation studies showed an increase in the proportion of relapse-free patients [75], a lower-thanexpected relapse rate [79], and a decrease in the number of gadolinium enhancing lesions on MRI $[78,80]$. Ongoing randomised, placebo controlled trials on high-dose vitamin D supplementation will clarify its effect on relapse rate. [201]

At present, it is uncertain whether vitamin $D$ is associated with the main functional disability hallmark in MS, disability progression. Cross-sectional studies have shown a negative correlation between $25(\mathrm{OH}) \mathrm{D}$ level and disability, yet causality is uncertain. $[73,103,202]$ Higher sunlight exposure, being the main source of vitamin $D$, has been associated with a decreased likelihood of reaching milestones on the expanded disability status scale (EDSS) in RRMS and also with a decreased risk ratio of reaching milestones on the patient determined disability scale (PDSS) in progressive MS. [203, 204] Conversely, no correlation between 25(OH)D levels and the MS severity scale (MSSS) or EDSS has been found in African Americans [205] and the negative correlation between recent EDSS progression and 25(OH)D levels has not been retained after correcting for baseline EDSS in a prospective Tasmanian study [73]. Small, high dose vitamin D supplementation studies have revealed a reduced proportion of EDSS progressive RRMS patients [75] and a trend towards reduced disability [80]. In interferon- $\beta$ (IFN$\beta$ ) treated or naive clinically isolated syndrome (CIS) patients, higher $25(\mathrm{OH}) \mathrm{D}$ levels, measured in the first months after diagnosis, predicted reduced disease activity and a slower rate of progression. [206]

The reduction of relapse risk associated with higher levels of $25(\mathrm{OH}) \mathrm{D}$ in the early, inflammatory course of the disease may be accompanied by a reduction of disability progression. $[207,208]$ Whether disability progression later on, may be predicted by vitamin D status is at present less clear. The answer to this is of great importance, since people with long standing MS constitute the largest proportion of patients and that number also includes the patients with progressive disease, who have the lowest 25(OH)D levels. [103] A low 25(OH)D status could be a target for intervention in these patients who have at present limited (RRMS) to no (progressive MS) therapeutic possibilities. 
To elucidate further the effect of vitamin $D$ status on disability progression, we conducted a retrospective, three-year follow-up study in which we assessed the predictive value of baseline $25(\mathrm{OH}) \mathrm{D}$ levels on relapse risk, EDSS disability, and EDSS progression in $554 \mathrm{MS}$ patients. We show that $25(\mathrm{OH}) \mathrm{D}$ status, within the physiological range, is just significantly associated with the occurrence of relapses in younger MS patients, but is not associated with disability or disability progression over the three years follow-up.

\section{Methods}

\section{Patients and study outcome measurements}

All patients with MS according to the original or 2005 revised McDonald criteria $[22,24]$ were eligible for inclusion in the cohort. They all visited the Academic MS Centre Limburg, the Netherlands in the period 2005 to 2013. This centre was based at the Maastricht University Medical Centre, Maastricht and is currently at the Zuyderland Medical Centre, Sittard. Both cities are located in the south of the Netherlands at $51^{\circ}$ latitude north. All patients visited the outpatient clinic for normal clinical care and were included when they had had a three year follow-up in EDSS [209] by the end of 2013, after a baseline serum 25(OH)D level measurement with corresponding EDSS within 6 months. During the visits to the outpatient clinic, relevant events regarding MS activity and progression were routinely registered. Clinical characteristics were recorded in all subjects according to the Dutch law on Medical Treatment Act (WGBO), the Personal Data Protection Act (Wbp) and the Code of Conduct for Health Research (Federa). [210] Data included age, sex, MS debut (date), MS phenotype [211] (RRMS, SPMS, PPMS; RRMS and SPMS patients were classified as RRMS-onset), date of MS diagnosis, start of progressive disease (date) if applicable, relapses (number and date), baseline serum 25(OH)D levels ( $\mathrm{nmol} / \mathrm{L}$ ), baseline EDSS, at least 1 follow-up EDSS more than 3 years after inclusion, information on $25(\mathrm{OH}) \mathrm{D}$ supplementation and disease modifying treatments (DMT). EDSS and the occurrence of relapses (defined as the development of new symptom(s) or aggravation of existing symptom(s) for at least 24 hours in a patient with stationary or improving disease course in the previous month [20]), were assessed by an experienced neurologist. Only EDSS during periods of remission (defined as having relapse free disease at least in the three months prior and half a month after measurement) were included. Patients who were included in high dose vitamin D supplementation (>800 IU, equal to $>20 \mu \mathrm{g}$ ) studies were excluded from the current study. Patients who were classified as having progressive relapsing MS were assigned to the SPMS group. The use of DMTs was highly variable between and within patients, because of treatment choices based on clinical patient care. Therefore, we were not able to include these data as an independent variable in our analysis.

Based on the inclusion criteria, 793 patients were deemed to be potential study subjects and their patient files were studied in detail. Eventually, 554 MS patients were included in the study (Figure S1). 


\section{5(OH)D measurement}

During the study period, serum 25(OH)D levels were measured by routine clinical analysis. Before 2008 ( $n=196$ samples), both a chemiluminescence immunoassay (CLIA) (Nichols Institute Diagnostics, California, USA) and a radioimmunoassay (Immunodiagnostics Systems, Boldon, UK) were used, due to a change in clinical diagnostic operating procedures. Both methods had a good inter-assay correlation. [103] After 2008 ( $n=358$ samples), a CLIA (LIAISON® $25 \mathrm{OH}$ Vitamin D TOTAL Assay, Diasorin, Saluggia, Italy) was used.

In accordance with other studies, $25(\mathrm{OH}) \mathrm{D}$ data were deseasonalised to correct for seasonal variation of $25(\mathrm{OH}) \mathrm{D}$ levels. $[73,212,213]$ The total of all consecutive $25(\mathrm{OH}) \mathrm{D}$ levels from all patients were used to model seasonal variation in our cohort according to the sinusoidal model described by van der Mei et al. [73] yt $=\beta 0+\beta 1 \sin (2 \pi t / 365)+\beta 2 \cos (2 \pi t / 365)$, where yt denotes serum $25(\mathrm{OH}) \mathrm{D}$ concentration, $t$ denotes the day of the year when the sample was collected, and $\beta j(j=0,1,2)$ are estimated regression coefficients. The adjusted 25(OH)D value was calculated by applying the deviation of an individual from the population mean at a time-point measured, on the population mean at $T=0$. The seasonal corrected $25(\mathrm{OH}) \mathrm{D}$ levels are referred to as vitamin D status.

\section{Statistical analysis}

SPSS software (SPSS Inc., version 20.0, Chicago, USA) was used to analyse the effect of the deseasonalised $25(\mathrm{OH}) \mathrm{D}$ status at baseline as the primary predictor of relapses during the three year follow-up period in RRMS-onset patients. This association was assessed by using either a logistic (yes/no) or ordinal logistic regressions $(0,1,2,3$ or more relapses over followup). Covariates included in these models were age at baseline (years), sex (M/F), disease duration (years), MS-phenotype (RRMS, SPMS), number of relapses in the three years prior to baseline, and EDSS at baseline. To answer the question whether the effects were MSphenotype dependent the second order interaction baseline deseasonalised $25(\mathrm{OH}) \mathrm{D}^{*} \mathrm{MS}$ phenotype, was investigated. Furthermore, potential relevant interactions with the primary outcome measure relapse rate, that were investigated were sex*age, sex*disease duration, age at baseline*baseline deseasonalised $25(\mathrm{OH}) \mathrm{D}$, baseline deseasonalised $25(\mathrm{OH}) \mathrm{D}$ *baseline EDSS and the interactions with pre-study relapse rate. Interactions that were not significantly relevant for the model and did not investigate our primary research question were omitted from the final analyses using stepwise backward modelling.

Repeated measures linear regression analyses were performed to investigate the role of baseline deseasonalised 25(OH)D in EDSS progression and to account for the correlation between repeated measurements within the same patients. Based on the best fit of the model to our data (-2LL (REML) and BIC), a model with Toeplitz covariance structure was used to model these correlations. The robustness of the final model was verified by applying different covariance structures. The outcome measure EDSS was analysed as a continuous variable [214] and was measured over equal spaces in time (trimesters). All EDSS data between baseline and three year follow-up were included in the analyses. Besides baseline deseasonalised $25(\mathrm{OH}) \mathrm{D}$ status, other covariates of potential interest that were included in 
the model were follow-up time (trimesters), age at baseline (years), sex (M/F), disease duration (years), baseline EDSS, and MS-phenotype (RRMS, SPMS, PPMS). To look at the effects on EDSS change, all first order interactions with time were investigated. Other relevant interactions that may affect the role of deseasonalised 25(OH)D levels on disease progression, were included: disease duration*sex, baseline deseasonalised $25(\mathrm{OH}) \mathrm{D}^{*}$ disease duration, baseline deseasonalised $25(\mathrm{OH}) \mathrm{D} * \mathrm{MS}$-phenotype, baseline EDSS*relapse rate pre-baseline, age at baseline*relapse rate pre study, disease duration*relapse rate pre study, sex*relapse rate pre-baseline and baseline EDSS*sex. Finally we also included the three way interaction baseline deseasonalised $25(\mathrm{OH}) \mathrm{D}^{*}$ time*MS-phenotype, to investigate the MS-phenotype dependent effects of vitamin $D$ on EDSS change. Interactions that were not significantly relevant for the model and did not investigate the relation between $25(\mathrm{OH}) \mathrm{D}$ and EDSS or EDSS progression were omitted from the analyses.

All of our tests were hypothesis driven. Three main outcome variables were tested: the relation of $25(\mathrm{OH}) \mathrm{D}$ status with relapse rate in three years follow-up, the relation with EDSS and with EDSS progression. Therefore the number of tests was minimal and correction for multiple testing was not applicable. A p-value of $<0.05$ was considered statistically significant.

\section{Results}

\section{Patient characteristics and deseasonalisation of 25(OH)D levels}

The total study cohort comprised 554 patients. Patient characteristics are shown in Table 1. The characteristics of this selected study population did not differ from the total MS population at the Academic MS Centre Limburg with respect to age at diagnosis, female/male ratio and MS-phenotype distribution (age at diagnosis $39.3 \pm 10.9$ year; $71.6 \%$ female, $28.4 \%$ male; distribution of MS phenotype 51.8\% RRMS, 34.1\% SPMS and 14.0\% PPMS). The model of the seasonal fluctuation of the 25(OH)D levels of the whole population had a mean of 59.2 $\mathrm{nmol} / \mathrm{L}$ and an average fluctuation between $50.6 \mathrm{nmol} / \mathrm{L}$ and $67.9 \mathrm{nmol} / \mathrm{L}$. Based on this data, the following formula was used for the deseasonalisation of all individual $25(\mathrm{OH}) \mathrm{D}$ data: deseasonalised $25(\mathrm{OH}) \mathrm{D}=$ crude $\left.25(\mathrm{OH}) \mathrm{D}-\left(-4.168^{*} \sin (2 \pi \mathrm{t} / 365)\right)-7.546 * \cos (2 \pi \mathrm{t} / 365)\right)$ (Figure 1).

Table 1. Characteristics of the selected study population of MS patients of the Academic MS Centre Limburg.

\begin{tabular}{|lll|}
\hline Population & Number & Percentage \\
\hline Number of patients & 554 & \\
\hline Age (years) & $46.7(11.6)$ & \\
\hline Sex (F/M) & $395 / 158$ & $71 \% / 29 \%$ \\
\hline MS phenotype & & \\
\hline \multicolumn{1}{|c|}{ RRMS } & 340 & $61.4 \%$ \\
\hline \multicolumn{1}{|l}{ SPMS } & 152 & $27.4 \%$ \\
\hline DPMS & 62 & $11.2 \%$ \\
\hline
\end{tabular}




\begin{tabular}{|c|c|c|}
\hline \multirow[t]{2}{*}{ since onset } & \multicolumn{2}{|l|}{$12.5(10.1)$} \\
\hline & \multicolumn{2}{|l|}{ \#9.7 (4.2-18.8) } \\
\hline \multirow[t]{2}{*}{ since diagnosis } & \multicolumn{2}{|l|}{$7.2(7.7)$} \\
\hline & \multicolumn{2}{|l|}{ \#4.2 (0.9-11.3) } \\
\hline \multicolumn{3}{|l|}{ Age (years) } \\
\hline at onset & \multicolumn{2}{|l|}{$34.2(10.4)$} \\
\hline at diagnosis & \multicolumn{2}{|l|}{$39.6(10.8)$} \\
\hline \multicolumn{3}{|l|}{ Number of pre-baseline relapses (in 3 years) } \\
\hline 0 & 196 & $35.4 \%$ \\
\hline 1 & 147 & $26.5 \%$ \\
\hline 2 & 104 & $18.8 \%$ \\
\hline 3 or more & 107 & $19.3 \%$ \\
\hline Baseline EDSS & \multicolumn{2}{|l|}{$4.0(2.0-6.0)$} \\
\hline$\leq 3.5$ & 274 & $49.5 \%$ \\
\hline $4.0-5.5$ & 129 & $23.2 \%$ \\
\hline$\geq 6.0$ & 151 & $27.3 \%$ \\
\hline EDSS after 3 years follow-up & \multicolumn{2}{|l|}{$4.0(2.5-6.5)$} \\
\hline$\leq 3.5$ & 228 & $41.2 \%$ \\
\hline $4.0-5.5$ & 140 & $25.3 \%$ \\
\hline$\geq 6.0$ & 186 & $33.6 \%$ \\
\hline \multicolumn{3}{|l|}{ Number of patients with EDSS progression after 3 years follow-up } \\
\hline$<1$ & 357 & $64.4 \%$ \\
\hline$\geq 1$ & 197 & $35.6 \%$ \\
\hline \multicolumn{3}{|l|}{ Baseline $25(\mathrm{OH}) \mathrm{D}(\mathrm{nmol} / \mathrm{L})$} \\
\hline crude & \multicolumn{2}{|l|}{$57.5(28.4)$} \\
\hline deseasonalised & \multicolumn{2}{|l|}{$56.7(28.0)$} \\
\hline \multicolumn{3}{|l|}{ Disease modifying treatment used most frequently during follow-up } \\
\hline none & 225 & $40.6 \%$ \\
\hline first line treatment (interferon, glatiramer acetate) & 193 & $34.8 \%$ \\
\hline second line treatment (natalizumab, fingolimod) & 40 & $7.2 \%$ \\
\hline $\begin{array}{l}\text { third line treatment (methotrexate, immunoglobulins, } \\
\text { mitoxantrone) }\end{array}$ & 75 & $13.5 \%$ \\
\hline Other & 6 & $1.0 \%$ \\
\hline Unknown & 15 & $2.7 \%$ \\
\hline \multicolumn{3}{|l|}{ Number of relapses during three year follow-up } \\
\hline 0 & 288 & $52.0 \%$ \\
\hline 1 & 130 & $23.5 \%$ \\
\hline 2 & 67 & $12.1 \%$ \\
\hline 3 or more & 69 & $12.5 \%$ \\
\hline
\end{tabular}

Data are provided as mean (SD) and as "median (Q1-Q3) in case of skewed distributions.

25(OH)D: 25-hydroxyvitamin D; EDSS: expanded disability status scale; F: female; M: male; PPMS: primary progressive multiple sclerosis; RRMS: relapsing remitting multiple sclerosis; SPMS: secondary progressive multiple sclerosis 


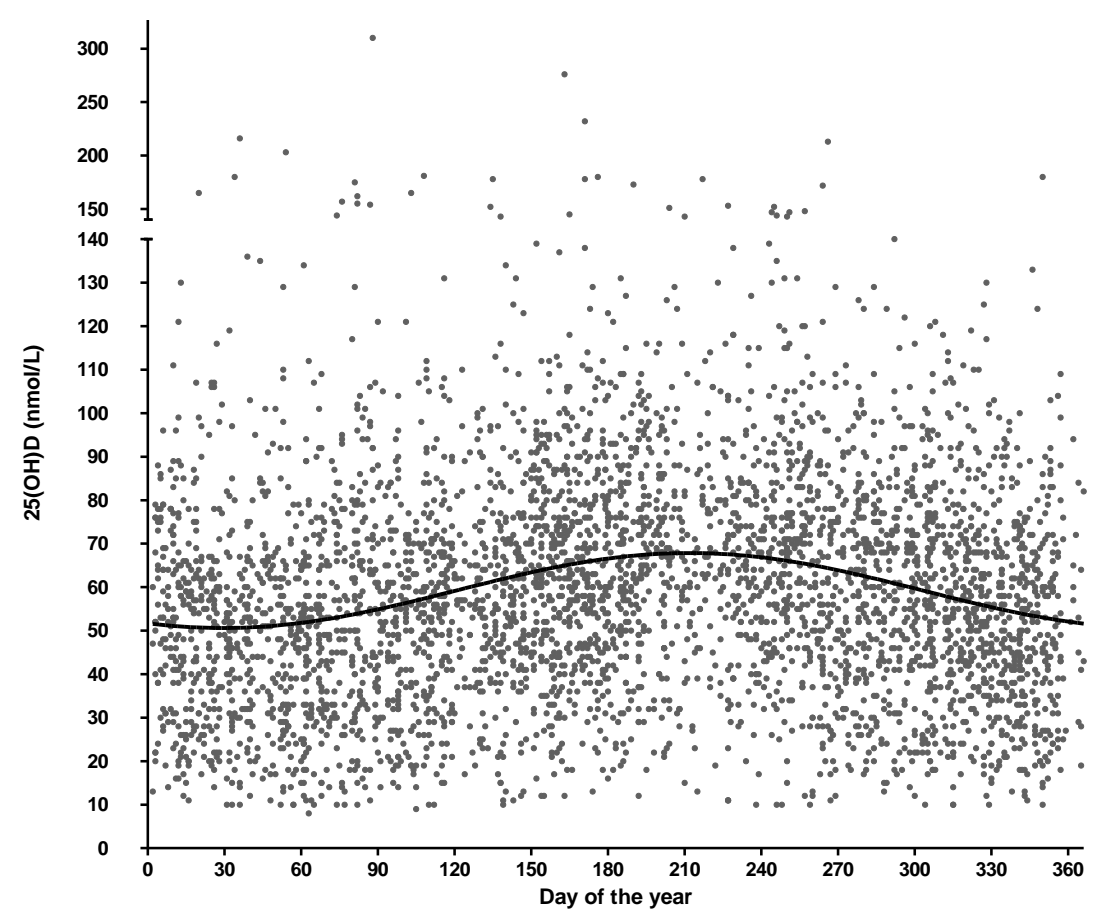

Figure 1. Seasonal fluctuation in $25(\mathrm{OH}) \mathrm{D}$ levels measured in the total Academic MS Centre Limburg MS population ( $n=429425(\mathrm{OH}) \mathrm{D}$ measurements).

Each dot represents a $25(\mathrm{OH}) \mathrm{D}$ measurement of an MS patient. Formula of curve: $25(\mathrm{OH}) \mathrm{D}=59.23$ $\left.\left.4.168^{*} \sin (2 \pi t / 365)\right)-7.546{ }^{*} \cos (2 \pi t / 365)\right)$.

25(OH)D: 25-hydroxyvitamin D

\section{Vitamin D status predicts the risk of relapses during three-year follow-up in younger RRMS-onset patients}

In the RRMS-onset cohort ( $n=490$, characteristics in Table S1), we studied the effect of baseline $25(\mathrm{OH}) \mathrm{D}$ level on relapse rate. Baseline deseasonalised $25(\mathrm{OH}) \mathrm{D}$ did not have an overall effect on the risk of getting relapses (yes/no) $(O R=0.96, C I O R=0.89-1.03, p=0.21$, Table 2A) or the number of relapses (Table S2) in the follow-up period of three years. However, the effect of $25(\mathrm{OH}) \mathrm{D}$ status on the risk of getting relapses was age dependent $(\mathrm{OR}=1.007, \mathrm{CI}$ $\mathrm{OR}=1.000-1.015, \mathrm{p}=0.047$, Table $2 \mathrm{~B}$ ), showing a low significant beneficial effect of deseasonalised baseline $25(\mathrm{OH}) \mathrm{D}$ status on relapses (yes/no) in RRMS-onset patients in the lowest age at baseline quartile $\leq 37.5$ year $(O R=0.872, C I O R=0.765-0.994, p=0.041$ in the most extensive model, Table 2C), no effect in MS patients aged between 37.5 and 54 year, and a disadvantageous effect in the MS patients aged $\geq 54$ year at baseline which was also just significant $(O R=1.430, C I O R=1.003-2.040 p=0.048$, Table 2C). Other predictors of relapses were age at baseline per se, EDSS at baseline and relapse rate pre-baseline, the last factor being disease duration dependent. 
Table 2. Association of vitamin D status and relapses (yes/no) during three year follow-up in RRMS-onset patients.

A Model 1: main variables

\section{Vitamin D and relapse risk in RRMS-onset population (logistic regression)}

\begin{tabular}{lllll} 
Parameter & B & $\begin{array}{l}\text { Odds } \\
\text { Ratio }\end{array}$ & 95\% CI OR & p-value \\
\hline Relapses (ref.= no) & & & & \\
\hline Baseline 25(OH)D (per $10 \mathrm{nmol} / \mathrm{L})$ deseasonalised & -0.045 & 0.956 & $0.892-1.025$ & 0.206 \\
\hline SPMS (ref. = RRMS) & -0.642 & 0.526 & $0.275-1.008$ & 0.053 \\
\hline Age at baseline (years) & -0.036 & 0.964 & $0.944-0.985$ & $0.001^{*}$ \\
\hline Duration of disease (years) & 0.024 & 1.024 & $0.994-1.055$ & 0.112 \\
\hline Sex (ref. = female) & -0.183 & 0.833 & $0.538-1.290$ & 0.413 \\
\hline EDSS baseline & 0.138 & 1.148 & $1.003-1.313$ & $0.045^{*}$ \\
\hline EDSS baseline ${ }^{2}$ (centred around mean of 3.8) & -0.093 & 0.912 & $0.869-0.956$ & $<0.001^{\star}$ \\
\hline Relapse rate 3 years pre-baseline & 0.243 & 1.275 & $1.107-1.467$ & $0.001^{*}$ \\
\hline
\end{tabular}

See for characteristics of the RRMS-onset study population Table S1

* significant independent variable at p<0.05; 25(OH)D: 25-hydroxyvitamin D; CI: confidence interval; EDSS: expanded disability status scale; OR: odds ratio; ref: reference category; RRMS: relapsing remitting multiple sclerosis; SPMS: secondary progressive multiple sclerosis

B Model 2: model 1 plus interaction terms

\begin{tabular}{|c|c|c|c|c|}
\hline Parameter & B & $\begin{array}{l}\text { Odds } \\
\text { Ratio }\end{array}$ & 95\% CI OR & p-value \\
\hline \multicolumn{5}{|l|}{ Relapses (ref. = no) } \\
\hline Baseline 25(OH)D (per $10 \mathrm{nmol} / \mathrm{L}$ ) deseasonalised & -0.333 & 0.717 & $0.530-0.970$ & $0.031^{*}$ \\
\hline $\begin{array}{l}\text { SPMS (ref. = RRMS)*Baseline } 25(\mathrm{OH}) \mathrm{D} \\
\text { (per } 10 \mathrm{nmol} / \mathrm{L} \text { ) deseasonalised }\end{array}$ & -0.090 & 0.914 & $0.764-1.094$ & 0.327 \\
\hline $\begin{array}{l}\text { Age at baseline (years)*Baseline } 25(\mathrm{OH}) \mathrm{D} \\
\text { (per } 10 \mathrm{nmol} / \mathrm{L} \text { ) deseasonalised }\end{array}$ & 0.007 & 1.007 & $1.000-1.0145$ & $0.047^{*}$ \\
\hline SPMS (ref. = RRMS) & -0.222 & 0.801 & $0.248-2.592$ & 0.712 \\
\hline Age at baseline (years) & -0.077 & 0.926 & $0.884-0.970$ & $0.001^{*}$ \\
\hline Duration of disease (years) & -0.010 & 0.990 & $0.951-1.030$ & 0.610 \\
\hline Sex (ref. = female) & -0.217 & 0.805 & $0.516-1.257$ & 0.340 \\
\hline EDSS baseline & 0.149 & 1.160 & $1.013-1.329$ & $0.032^{*}$ \\
\hline EDSS baseline $^{2}$ (centred around mean of 3.8) & -0.093 & 0.911 & $0.868-0.957$ & $<0.001^{*}$ \\
\hline Relapse rate 3 years pre-baseline & 0.032 & 1.033 & $0.843-1.265$ & 0.757 \\
\hline
\end{tabular}

Corrected for "duration of disease (years)*relapse rate 3 years pre-baseline" B 0.029, OR 1.030, $p=0.012$

See for characteristics of the RRMS-onset study population Table S1

* significant independent variable at p<0.05; 25(OH)D: 25-hydroxyvitamin D; CI: confidence interval; EDSS: expanded disability status scale; ref:: reference category; OR: odds ratio; RRMS: relapsing remitting multiple sclerosis; SPMS: secondary progressive multiple sclerosis 
C Effect of vitamin D on relapses (yes/no) in different age categories $(n=123$ per quartile) at baseline Vitamin D and relapse risk in RRMS-onset population (logistic regression) Effect of baseline 25(OH)D (per $10 \mathrm{nmol} / \mathrm{L}$ ) deseasonalised

\begin{tabular}{|c|c|c|c|c|}
\hline Age category & B & Odds Ratio & $95 \%$ CI OR & p-value \\
\hline \multicolumn{5}{|l|}{ Q1: <37.5 year } \\
\hline Simple model & -0.109 & 0.897 & $0.797-1.009$ & $0.069^{\star}$ \\
\hline Model 1 & -0.120 & 0.890 & $0.786-1.007$ & $0.065^{\star}$ \\
\hline Model 2 (adjusted) & -0.137 & 0.872 & $0.765-0.994$ & $0.041^{\star}$ \\
\hline \multicolumn{5}{|l|}{ Q2: 37.5-46.2 year } \\
\hline Simple model & -0.065 & 0.937 & $0.824-1.066$ & 0.323 \\
\hline Model 1 & -0.040 & 0.961 & $0.835-1.106$ & 0.581 \\
\hline Model 2 (adjusted) & -0.024 & 0.977 & $0.834-1.144$ & 0.770 \\
\hline \multicolumn{5}{|l|}{ Q3: 46.2-54.0 year } \\
\hline Simple model & -0.056 & 0.946 & $0.836-1.070$ & 0.376 \\
\hline Model 1 & -0.075 & 0.928 & $0.806-1.068$ & 0.296 \\
\hline Model 2 (adjusted) & 0.013 & 1.013 & $0.855-1.200$ & 0.883 \\
\hline \multicolumn{5}{|l|}{ Q4: >54.0 year } \\
\hline Simple model & 0.203 & 1.226 & $1.030-1.460$ & $0.022^{\star}$ \\
\hline Model 1 & 0.140 & 1.151 & $0.943-1.404$ & 0.166 \\
\hline Model 2 (adjusted) & 0.358 & 1.430 & $1.003-2.040$ & $0.048^{\star}$ \\
\hline
\end{tabular}

See for characteristics of the RRMS-onset study population Table S1

Simple model includes only baseline $25(\mathrm{OH}) \mathrm{D}$ deseasonalised as covariate.

Model 1 includes baseline 25(OH)D deseasonalised, MS phenotype, age at baseline, duration of disease, sex, EDSS baseline, relapse rate three years pre-baseline, and EDSS baseline ${ }^{2}$ (centred) as covariates.

Model 2 (adjusted) same as Model 1 but including MS phenotype*baseline 25(OH)D deseasonalised, and duration of disease*pre-baseline relapse rate as covariates and excluding age at baseline*baseline $25(\mathrm{OH}) \mathrm{D}$ deseasonalised

* significant effect of baseline 25(OH)D deseasonalised at p<0.05; 25(OH)D: 25-hydroxyvitamin D; CI: confidence interval; OR: odds ratio; RRMS: relapsing remitting multiple sclerosis; $Q$ : quartile

\section{Vitamin D status does not predict disability and disability progression in multiple scle- rosis patients over three year follow-up}

Baseline deseasonalised 25(OH)D status did not have any overall effect on EDSS $(\beta=-0.003$, $p=0.615$ (Table S3A)) or EDSS change over three year follow up $(\beta=-0.002, p=0.790$ (Table $3 \mathrm{~A}$ ) in the simple model and $\beta=-0.011, p=0.113$ in the more extensive model (table $3 \mathrm{~B}$ )). Also after correction for MS-phenotype, 25(OH)D did not predict EDSS change over time $(\beta=0.021, p=0.198$ for SPMS and $\beta=0.013, p=0.621$ for PPMS with RRMS as reference, table S3B) which was confirmed by an MS phenotype subgroup analyses (data not shown). MS phenotype at baseline, age at baseline, EDSS at baseline and relapse rate three years prebaseline were the most important predictors in the model for EDSS change over time (table $3 B$ ). In addition, whether patients had stable or progressive disease ( $\geq 1$ EDSS increase over three year follow-up) was not dependent on baseline $25(\mathrm{OH}) \mathrm{D}$ status in a logistic regression model $(\mathrm{OR}=1.021$, CI OR=0.952-1.095, $\mathrm{p}=0.560)$. 
Table 3. Effect of vitamin D status on EDSS over 3 year follow-up according to repeated measures linear regression analyses with Toeplitz covariance structure.

\section{A Model 1: main variables}

\begin{tabular}{|c|c|c|c|}
\hline \multicolumn{4}{|c|}{$\begin{array}{l}\text { Repeated measures linear regression analyses of longitudinal EDSS data with Toeplitz covari- } \\
\text { ance structure }\end{array}$} \\
\hline Parameter & $\boldsymbol{\beta}$ & $95 \%$ CI $\beta$ & p-value \\
\hline Baseline 25(OH)D (per $10 \mathrm{nmol} / \mathrm{L}$ ) deseasonalised & -0.002 & $-0.014-0.0100$ & 0.733 \\
\hline $\begin{array}{l}\text { Baseline } 25(\mathrm{OH}) \mathrm{D} \text { deseasonalised*time since baseline } \\
\text { (per } 10 \mathrm{nmol} / \mathrm{L} \text { per year) }\end{array}$ & -0.002 & $-0.016-0.012$ & 0.790 \\
\hline \multicolumn{4}{|l|}{ MS phenotype at baseline (ref. = RRMS) } \\
\hline SPMS & 0.049 & $-0.054-0.152$ & 0.353 \\
\hline PPMS & 0.070 & $-0.039-0.180$ & 0.208 \\
\hline Time since baseline (years) & 0.156 & $0.072-0.244$ & $<0.001^{*}$ \\
\hline Age at baseline (years) & 0.002 & $-0.001-0.006$ & 0.148 \\
\hline Duration of disease (years) & 0.001 & $-0.004-0.006$ & 0.809 \\
\hline Sex (ref. = female) & 0.015 & $-0.053-0.082$ & 0.670 \\
\hline EDSS baseline & 0.962 & $0.941-0.983$ & $<0.001^{*}$ \\
\hline EDSS baseline $^{2}$ (centred around mean of 3.8) & 0.010 & $0.002-0.017$ & $0.012^{*}$ \\
\hline Relapse rate 3 years pre-baseline & 0.005 & $-0.017-0.026$ & 0.679 \\
\hline
\end{tabular}

* significant independent variable at p<0.05; 25(OH)D: 25-hydroxyvitamin D; CI: confidence interval; EDSS: expanded disability status scale; PPMS: primary progressive multiple sclerosis; ref: reference category; RRMS: relapsing remitting multiple sclerosis; SPMS: secondary progressive multiple sclerosis

\section{B Model 2: Model 1 plus two way interaction terms}

Repeated measures linear regression analyses of longitudinal EDSS data with Toeplitz covariance structure

\begin{tabular}{|c|c|c|c|}
\hline Parameter & $\beta$ & $95 \% \mathrm{CI} \beta$ & p-value \\
\hline Baseline 25(OH)D (per $10 \mathrm{nmol} / \mathrm{L}$ ) deseasonalised & -0.0037 & $-0.017-0.010$ & 0.595 \\
\hline $\begin{array}{l}\text { Baseline } 25(\mathrm{OH}) \mathrm{D} \text { deseasonalised*time since baseline } \\
\text { (per } 10 \mathrm{nmol} / \mathrm{L}^{*} \text { year) }\end{array}$ & -0.011 & $-0.024-0.003$ & 0.113 \\
\hline \multicolumn{4}{|l|}{$\begin{array}{l}\text { MS phenotype at baseline (ref. = RRMS)*baseline } 25(\mathrm{OH}) \mathrm{D} \\
\text { (per } 10 \mathrm{nmol} / \mathrm{L} \text { ) deseasonalised }\end{array}$} \\
\hline SPMS & 0.017 & $-0.008-0.043$ & 0.175 \\
\hline PPMS & 0.014 & $-0.025-0.053$ & 0.479 \\
\hline \multicolumn{4}{|l|}{ MS phenotype at baseline (ref. = RRMS) } \\
\hline SPMS & -0.132 & $-0.307-0.043$ & 0.139 \\
\hline PPMS & -0.087 & $-0.330-0.155$ & 0.480 \\
\hline Time since baseline (years) & 0.415 & $0.208-0.623$ & $<0.001^{*}$ \\
\hline Age at baseline (years) & 0.0004 & $\begin{array}{l}-0.0031- \\
0.0039\end{array}$ & 0.831 \\
\hline Duration of disease (years) & 0.003 & $-0.002-0.008$ & 0.285 \\
\hline Sex (ref. = female) & 0.092 & $0.001-0.183$ & $0.048^{*}$ \\
\hline EDSS baseline & 1.012 & $0.989-1.035$ & $<0.001^{*}$ \\
\hline EDSS baseline ${ }^{2}$ (centred around mean of 3.8) & 0.011 & $0.003-0.018$ & $0.004^{*}$ \\
\hline Relapse rate 3 years pre-baseline & 0.016 & $-0.007-0.040$ & 0.173 \\
\hline
\end{tabular}




\begin{tabular}{|c|c|c|c|}
\hline \multicolumn{4}{|l|}{$\begin{array}{l}\text { MS phenotype at baseline (ref. = RRMS)*time since base- } \\
\text { line (years) }\end{array}$} \\
\hline SPMS & 0.237 & $0.119-0.355$ & $<0.001^{*}$ \\
\hline PPMS & 0.170 & $0.043-0.297$ & $0.009^{*}$ \\
\hline Age at baseline (years)*time since baseline (years) & 0.005 & $0.002-0.009$ & $0.006^{*}$ \\
\hline EDSS baseline ${ }^{\star}$ time since baseline (years) & -0.130 & $-0.154--0.106$ & $<0.001^{*}$ \\
\hline $\begin{array}{l}\text { Relapse rate } 3 \text { years pre-baseline*time since baseline } \\
\text { (trimesters) }\end{array}$ & -0.031 & $-0.058--0.004$ & $0.023^{*}$ \\
\hline
\end{tabular}

Corrected for duration of disease (years) ${ }^{*} \operatorname{sex~B~}-0.010, p=0.029$

* significant independent variable at p<0.05; 25(OH)D: 25-hydroxyvitamin D; CI: confidence interval; EDSS: expanded disability status scale; PPMS: primary progressive multiple sclerosis; ref.: reference category; RRMS: relapsing remitting multiple sclerosis; SPMS: secondary progressive multiple sclerosis

\section{Discussion}

In this study, we retrospectively assessed the relation between baseline 25(OH)D status and EDSS progression over three year in an unselected, real life, clinical MS population. Disease disability per se, as expressed by EDSS, was not affected by baseline 25(OH)D levels and also the accumulation of disability over time was not correlated with baseline 25(OH)D status. In our population there was a strong trend towards $25(\mathrm{OH}) \mathrm{D}$ status predicting the occurrence of relapses, but only in MS patients in the youngest and the oldest age groups.

There are several mechanisms which support the hypothesis that a higher vitamin D status may reduce disability progression both in early MS patients with inflammatory disease and later on in established or progressive MS. First of all by the proposed immunomodulatory effects of vitamin D [147], which could prevent relapses in RRMS-onset patients, thereby preventing accumulation of tissue damage. It has been shown that recovery, post relapse, will be incomplete in a substantial proportion of about $50 \%$ of the patients, leading to an increased EDSS. [207] In the present study, we indeed showed that vitamin D status most probably can predict the occurrence of relapses in younger MS patients. Furthermore, vitamin $D$ could have a direct beneficial effect on the brain in late MS, by either neuroprotective effects, maintaining the blood brain barrier integrity, decreasing axonal damage or improving remyelination. [82-84] Additionally, the vitamin D responsiveness of the CNS may be increased due to neuroinflammation. [82] Our data do not support these hypotheses, though. However, they do confirm the results found by van der Mei et al. [73] and LøkenAmsrud et al. [215] who were also unable to detect a significant association of vitamin D status with EDSS progression, whether corrected for baseline EDSS or not. Soilu-Hanninen et al. [80] found (a trend towards) a relation between 25(OH)D levels and EDSS in their supplementation study in which patients with supplementation had a mean $25(\mathrm{OH}) \mathrm{D}$ level of 110 (67-163) nmol/L after 12 months. However, our patient population did not receive high-dose supplementation, was larger, and the follow up was longer. Furthermore, while most vitamin $\mathrm{D}$ association studies in MS were performed in selected patient cohorts, we deliberately have chosen for an unselected, real life MS population, in order to obtain robust results that can 
be used in clinical practice. In contrast to what we found in our unselected, clinical MS patient population, Ascherio et al. [206] showed that in CIS patients a $50 \mathrm{nmol} / \mathrm{L}$ increase in $25(\mathrm{OH}) \mathrm{D}$ levels was associated with a reduction of 0.16 EDSS points per year. This change was lower in the highest vitamin $D$ ranges. Altogether this may indicate that vitamin $D$ indeed plays a role in the onset and disease activity in young, early MS patients with inflammatory disease, but not at a later stage in the progression of disability. It has been suggested that once a clinical threshold of 4 EDSS points is reached, relapses no longer have any effect on the progression of disability. $[208,216]$ This might explain why the $25(\mathrm{OH}) \mathrm{D}$ status in young MS patients is related to the occurrence of relapses but did not show any overall association with disability progression.

Although we could not demonstrate any effect of vitamin D status on EDSS progression, also established and often used treatments in MS do not have an immense effect on EDSS progression. First line medication, such as IFN- $\beta$, has been shown to decrease EDSS in RRMS by 0.7 points in high dose and by 0.1 points in low doses in 24 months. [217] The pooled effect size in a meta-analysis was non-significant over 24 months and IFN- $\beta$ was also not associated with a change in disability progression in a big retrospective cohort study with a minimum follow-up of 4 years. [218, 219] Also in SPMS, no significant differences on the EDSS change were found between low dose IFN- $\beta 1 a$ and high dose IFN- $\beta 1 a$. [220] Glatiramer acetate was associated with an EDSS change of $0.6( \pm 2.0)$ over 15 years while withdrawn patients had a mean change of $1.0( \pm 1.7)$. [221] With natalizumab the mean EDSS-change was comparable for those patients who had ongoing treatment and the patients who withdrew (mean change 0.05 vs. 0.07). Over nearly 5 years, mean EDSS remained stable in both groups, while the difference between placebo and natalizumab remained significant (3.15 vs. 2.79). [222]

There are certain limitations in this study. First of all, this was a retrospective study and baseline $25(\mathrm{OH}) \mathrm{D}$ levels and EDSS were measured in a clinical setting, unrelated in timing to disease onset or diagnosis and not fixed to study visits. Second, it investigated the effect of $25(\mathrm{OH}) \mathrm{D}$ status in a, although clinically relevant and unselected, heterogeneous population, which might have led to a biased estimate of the true effect size. Third, the follow-up in this study was limited to a three year follow-up. Fourth, a majority of patients took low dose vitamin D supplementation (400/800 IU/day, equal to $10-20 \mu \mathrm{g} /$ day), which might have underestimated the outcomes. Vitamin $D$ status showed a regression to the mean over three year follow up (data not shown), which has also been described earlier. [206, 223] Other than the effect of supplementation, this regression to the mean might be due to lifestyle changes of the MS patients. Other important co-factors such as BMI, smoking habits and the use of DMTs could not be included. The fact that $25(\mathrm{OH}) \mathrm{D}$ levels were measured by different assays before and after 2008, did not change the vitamin D effect on disease activity and disease progression as was tested by adding this factor as a covariate to the models. Finally, EDSS as an outcome measure is not the ultimate measure with significant intra- and interrater differences and a non-linear course. However, it is the only frequently used measure available to monitor disability. 
While it was not possible to detect any effect of vitamin D status on disability progression, it might still be possible that (high dose) vitamin D supplementation is beneficial in MS patients with long standing and progressive disease. We examined 25(OH)D levels in the physiological range in patients taking only low doses of vitamin $D$ supplementation. This might have underestimated the effect of $25(\mathrm{OH}) \mathrm{D}$ status on EDSS (progression), but it might also be that high dose supplementation is needed to exert its effects on the immune system and the brain. Secondly, supplementation might be beneficial particularly in patients with the lowest $25(\mathrm{OH}) \mathrm{D}$ status. Finally, we were not able to take into account all the treatments patients received and, therefore, could not investigate the possible add-on or synergistic effects that vitamin D might have, as has been suggested for IFN- $\beta$ and fingolimod. [223226] Noticeably, this presumed interaction between vitamin $D$ and IFN- $\beta$ could not be reproduced by others. $[215,227]$

To date, evidence that vitamin D status affects disability progression in MS patients could not be found. So far, the small effects of vitamin D on disease activity and disability in MS seem to be mainly visible in well selected homogeneous subgroups of MS patients, which makes translation to the MS population in the daily clinical practice difficult. Long term follow-up studies of high-dose vitamin $D$ supplementation have to confirm these results. Before these data are available, though, it might be worthwhile considering preventive vitamin D supplementation in young (CIS and early) MS patients. 


\section{Supporting information}

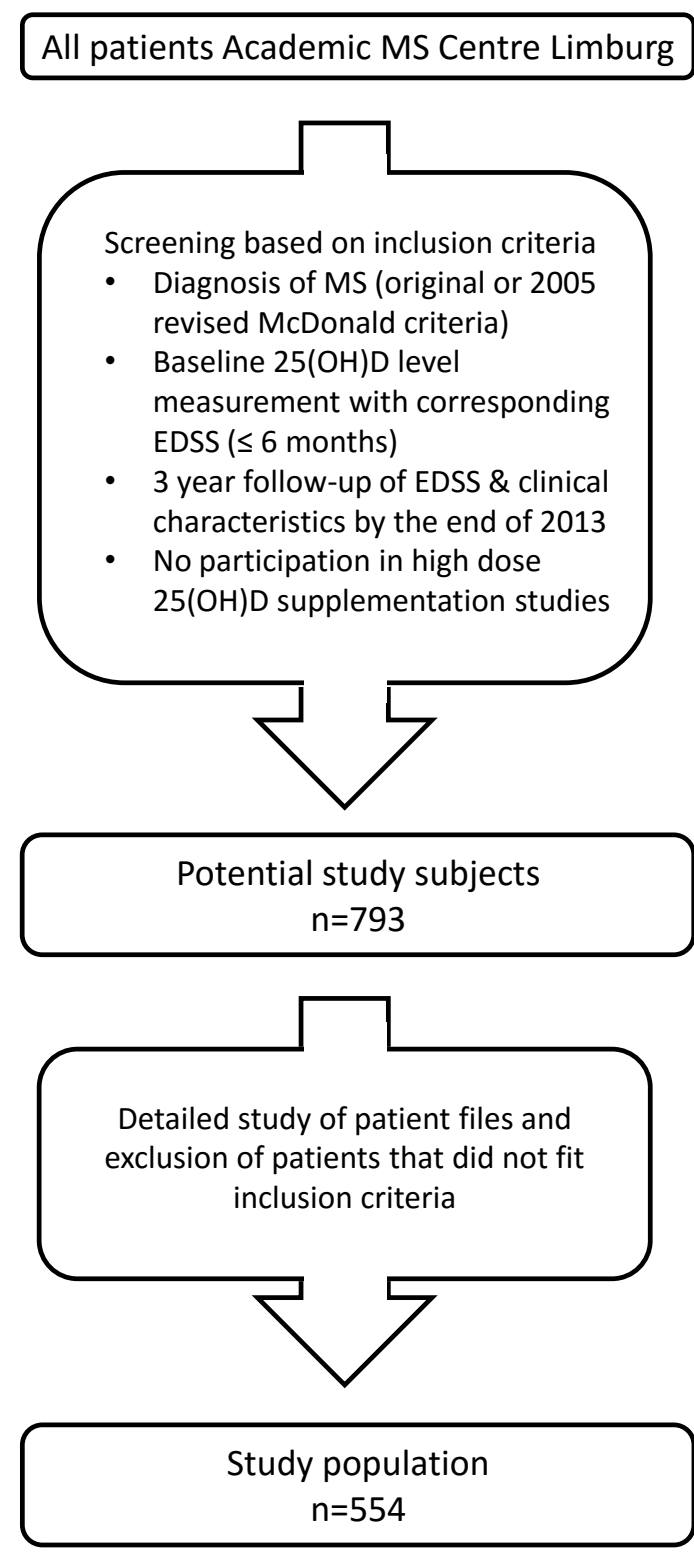

Figure S1. Flow chart inclusion of study patients.

25(OH)D: 25-hydroxyvitamin D; EDSS: expanded disability status scale; MS: multiple sclerosis; $n=n u m b e r$ 
Table S1. Characteristics of the RRMS-onset study population of MS patients of the Academic MS Centre Limburg.

\begin{tabular}{|c|c|c|}
\hline Study Population & Number & Percentage \\
\hline Number of patients & 492 & \\
\hline Age (years) & $45.9(11.6)$ & \\
\hline $\operatorname{Sex}(F / M)$ & $362 / 129$ & $73.6 \% / 26.2 \%$ \\
\hline \multicolumn{3}{|l|}{ MS phenotype } \\
\hline RRMS & 340 & $69.1 \%$ \\
\hline SPMS & 152 & $30.9 \%$ \\
\hline \multicolumn{3}{|l|}{ Disease duration (years) } \\
\hline \multirow[t]{2}{*}{ since onset } & $12.7(10.2)$ & \\
\hline & \#10.2 (4.2-19.2) & \\
\hline \multirow[t]{2}{*}{ since diagnosis } & $7.5(7.8)$ & \\
\hline & \#4.5 (1.1-11.8) & \\
\hline \multicolumn{3}{|l|}{ Age (years) } \\
\hline at onset & $33.2(10.0)$ & \\
\hline at diagnosis & $38.4(10.5)$ & \\
\hline \multicolumn{3}{|c|}{ Number of pre-baseline relapses during three year } \\
\hline 0 & 151 & $30.7 \%$ \\
\hline 1 & 138 & $28.0 \%$ \\
\hline 2 & 97 & $19.7 \%$ \\
\hline 3 or more & 106 & $21.5 \%$ \\
\hline \multicolumn{3}{|l|}{ Baseline EDSS } \\
\hline$\leq 3.5$ & 254 & $51.6 \%$ \\
\hline $4.0-5.5$ & 108 & $22.0 \%$ \\
\hline$\geq 6.0$ & 130 & $26.4 \%$ \\
\hline \multicolumn{3}{|l|}{ Baseline 25(OH)D (nmol/L) } \\
\hline crude & $58.0(28.9)$ & \\
\hline deseasonalised & $57.4(28.5)$ & \\
\hline \multicolumn{3}{|c|}{ Number of post baseline relapses during three year } \\
\hline 0 & 232 & $47.3 \%$ \\
\hline 1 & 124 & $25.3 \%$ \\
\hline 2 & 67 & $13.7 \%$ \\
\hline 3 or more & 67 & $13.7 \%$ \\
\hline
\end{tabular}

Data are provided as mean (SD) and as "median (Q1-Q3) in case of skewed distributions.

25(OH)D: 25-hydroxyvitamin D; EDSS: expanded disability status scale; F: female; M: male; PPMS: primary progressive multiple sclerosis; RRMS: relapsing remitting multiple sclerosis; SPMS: secondary progressive multiple sclerosis 
Table S2. Association of vitamin D status and relapses $(0,1,2,3$ or more) during three year follow-up in RRMSonset patients.

A Model 1: main variables

Vitamin D and relapse risk in RRMS-onset population (ordinal logistic regression)

\begin{tabular}{lllll} 
Parameter & $\boldsymbol{\beta}$ & $\begin{array}{l}\text { Odds } \\
\text { Ratio }\end{array}$ & 95\% CI OR & p-value \\
\hline Baseline 25(OH)D (per $10 \mathrm{nmol} / \mathrm{L}$ ) deseasonalised & -0.050 & 0.952 & $0.895-1.012$ & 0.117 \\
SPMS (ref. = RRMS) & -0.728 & 0.483 & $0.269-0.867$ & $0.015^{*}$ \\
\hline Age at baseline (years) & -0.035 & 0.966 & $0.948-0.984$ & $<0.001^{*}$ \\
Duration of disease (years) & 0.017 & 1.017 & $0.991-1.045$ & 0.201 \\
Sex (ref. = female) & -0.137 & 0.872 & $0.586-1.296$ & 0.499 \\
\hline EDSS baseline & 0.152 & 1.164 & $1.034-1.310$ & $0.012^{\star}$ \\
\hline EDSS baseline ${ }^{2}$ (centred around mean of 3.8) & -0.078 & 0.925 & $0.886-0.967$ & $0.001^{*}$ \\
\hline Relapse rate 3 years pre-baseline & 0.252 & 1.287 & $1.142-1.450$ & $<0.001^{*}$ \\
\hline
\end{tabular}

Test of parallel lines: $p=0.964$

See for characteristics of the RRMS-onset study population Table S1

* significant independent variable at $p<0.05 ; 25(\mathrm{OH}) D$ : 25-hydroxyvitamin $D ; C I$ : confidence interval; EDSS: expanded disability status scale; OR: odds ratio; ref: reference category; RRMS: relapsing remitting multiple sclerosis; SPMS: secondary progressive multiple sclerosis

B Model 2: Model 1 plus interaction terms

\begin{tabular}{|c|c|c|c|c|}
\hline Parameter & $\beta$ & $\begin{array}{l}\text { Odds } \\
\text { Ratio }\end{array}$ & 95\% CI OR & p-value \\
\hline Baseline 25(OH)D (per $10 \mathrm{nmol} / \mathrm{L}$ ) deseasonalised & -0.041 & 0.960 & $0.895-1.029$ & 0.249 \\
\hline $\begin{array}{l}\text { SPMS (ref. = RRMS) }{ }^{\star} \text { Baseline 25(OH)D (per } 10 \\
\mathrm{nmol} / \mathrm{L} \text { ) deseasonalised }\end{array}$ & -0.022 & 0.998 & $0.983-1.013$ & 0.770 \\
\hline SPMS (ref. = RRMS) & -0.725 & 0.484 & $0.184-1.273$ & 0.142 \\
\hline Age at baseline (years) & -0.034 & 0.966 & $0.948-0.985$ & $<0.001^{\star}$ \\
\hline Duration of disease (years) & -0.012 & 0.988 & $0.954-1.024$ & 0.519 \\
\hline Sex (ref. = female) & -0.136 & 0.872 & $0.586-1.300$ & 0.502 \\
\hline EDSS baseline & 0.164 & 1.178 & $1.046-1.327$ & $0.007^{*}$ \\
\hline EDSS baseline $^{2}$ (centred around mean of 3.8) & -0.076 & 0.927 & $0.887-0.969$ & $0.001^{*}$ \\
\hline Relapse rate 3 years pre-baseline & 0.093 & 1.097 & $0.924-1.304$ & 0.290 \\
\hline
\end{tabular}

Test of parallel lines: $p=0.925$

Corrected for duration of disease (years)* ${ }^{*}$ lapse rate 3 years pre-baseline B 0.020, OR 1.020, $p=0.017$

See for characteristics of the RRMS-onset study population Table S1

* significant independent variable at p<0.05; 25(OH)D: 25-hydroxyvitamin $D ; C I$ : confidence interval; EDSS: expanded disability status scale; OR: odds ratio; ref: reference category; RRMS: relapsing remitting multiple sclerosis; SPMS: secondary progressive multiple sclerosis 
Table S3. Additional analyses of the effect of vitamin D status on EDSS over 3 year follow-up according to repeated measures linear regression analyses with Toeplitz covariance structure.

\section{A Effect on EDSS independent of time}

\begin{tabular}{|c|c|c|c|}
\hline \multicolumn{4}{|c|}{$\begin{array}{l}\text { Repeated measures linear regression analyses of longitudinal EDSS data with Toeplitz covari } \\
\text { ance structure }\end{array}$} \\
\hline Parameter & $\beta$ & $95 \%$ CI $\beta$ & p-value \\
\hline Baseline 25(OH)D (per $10 \mathrm{nmol} / \mathrm{L}$ ) deseasonalised & -0.003 & $-0.014-0.008$ & 0.615 \\
\hline \multicolumn{4}{|l|}{ MS phenotype at baseline (ref. = RRMS) } \\
\hline SPMS & 0.049 & $-0.054-0.152$ & 0.353 \\
\hline PPMS & 0.070 & $-0.039-0.180$ & 0.208 \\
\hline Time since baseline (years) & 0.147 & $0.109-0.185$ & $<0.001^{*}$ \\
\hline Age at baseline (years) & 0.002 & $-0.001-0.006$ & 0.148 \\
\hline Duration of disease (years) & 0.001 & $-0.004-0.006$ & 0.810 \\
\hline Sex (ref. = female) & 0.015 & $-0.055-0.082$ & 0.671 \\
\hline EDSS baseline & 0.962 & $0.941-0.983$ & $<0.001^{*}$ \\
\hline EDSS baseline ${ }^{2}$ (centred around mean of 3.8) & 0.097 & $0.002-0.017$ & $0.012^{*}$ \\
\hline Relapse rate 3 years pre-baseline & 0.005 & $-0.017-0.026$ & 0.677 \\
\hline
\end{tabular}

* significant independent variable at p<0.05; 25(OH)D: 25-hydroxyvitamin D; CI: confidence interval; EDSS: expanded disability status scale; PPMS: primary progressive multiple sclerosis; ref.: reference category; RRMS: relapsing remitting multiple sclerosis; SPMS: secondary progressive multiple sclerosis

B Model 3: Model 2 (see Table 3B) plus three way interaction terms

\begin{tabular}{|c|c|c|c|}
\hline \multicolumn{4}{|c|}{$\begin{array}{l}\text { Repeated measures linear regression analyses of longitudinal EDSS data with Toeplitz covari } \\
\text { ance structure }\end{array}$} \\
\hline Parameter & $\beta$ & $95 \% \mathrm{CI} \beta$ & p-value \\
\hline Baseline 25(OH)D (per $10 \mathrm{nmol} / \mathrm{L}$ ) deseasonalised & -0.001 & $-0.016-0.013$ & 0.849 \\
\hline $\begin{array}{l}\text { Baseline } 25(\mathrm{OH}) \mathrm{D} \text { deseasonalisedtime since baseline } \\
\text { (per } 10 \mathrm{nmol} / \mathrm{L} \text { per year) }\end{array}$ & -0.004 & $-0.008--0.000$ & $0.041^{\star}$ \\
\hline $\begin{array}{l}\text { Baseline } 25(\mathrm{OH}) \mathrm{D} \text { deseasonalised*MS phenotype at baseline } \\
\text { (ref. = RRMS) (per } 10 \mathrm{nmol} / \mathrm{L})\end{array}$ & 0 & & \\
\hline SPMS & 0.009 & $-0.035-0.053$ & 0.532 \\
\hline PPMS & 0.009 & $-0.035-0.053$ & 0.689 \\
\hline \multicolumn{4}{|l|}{$\begin{array}{l}\text { Baseline } 25(\mathrm{OH}) \mathrm{D} \text { deseasonalised }{ }^{\star} \mathrm{MS} \text { phenotype at baseline } \\
\text { (ref. = RRMS)*time since baseline (per } 10 \mathrm{nmol} / \mathrm{L} \text { per year) }\end{array}$} \\
\hline SPMS & 0.021 & $-0.011-0.001$ & 0.198 \\
\hline PPMS & 0.013 & $-0.039-0.066$ & 0.621 \\
\hline \multicolumn{4}{|l|}{ MS phenotype at baseline (ref. = RRMS) } \\
\hline SPMS & -0.087 & $-0.274-0.101$ & 0.363 \\
\hline PPMS & -0.059 & $-0.323-0.206$ & 0.664 \\
\hline Time since baseline (years) & 0.448 & $0.235-0.661$ & $<0.001^{\star}$ \\
\hline Age at baseline (years) & 0.000 & $-0.0031-0.0038$ & 0.838 \\
\hline Duration of disease (years) & 0.003 & $-0.002-0.008$ & 0.288 \\
\hline Sex (ref. = female) & 0.092 & $0.001-0.183$ & $0.046^{\star}$ \\
\hline EDSS baseline & 1.012 & $0.989-1.035$ & $<0.001^{*}$ \\
\hline
\end{tabular}




\begin{tabular}{|c|c|c|c|}
\hline EDSS baseline ${ }^{2}$ (centred around mean of 3.8) & 0.011 & $0.003-0.018$ & $0.004^{*}$ \\
\hline Relapse rate 3 years pre-baseline & 0.016 & $-0.008-0.040$ & 0.186 \\
\hline \multicolumn{4}{|l|}{$\begin{array}{l}\text { MS phenotype at baseline }(\text { ref. }=\text { RRMS })^{\star} \text { time since baseline } \\
\text { (years) }\end{array}$} \\
\hline SPMS & 0.128 & $-0.076-0.331$ & 0.218 \\
\hline PPMS & 0.098 & $-0.210-0.405$ & 0.534 \\
\hline Age at baseline (years)*time since baseline (years) & 0.005 & $0.002-0.009$ & $0.006^{*}$ \\
\hline EDSS baseline ${ }^{\star}$ time since baseline (years) & -0.130 & $-0.154--0.105$ & $<0.001^{\star}$ \\
\hline Relapse rate 3 years pre-baseline $e^{\star}$ time since baseline (years) & -0.030 & $-0.057--0.003$ & $0.029^{*}$ \\
\hline
\end{tabular}

Corrected for duration of disease (years) ${ }^{*} \operatorname{sex~} B-0.010, p=0.027$

* significant independent variable at p<0.05; 25(OH)D: 25-hydroxyvitamin D; CI: confidence interval; EDSS: expanded disability status scale; PPMS: primary progressive multiple sclerosis; ref.: reference category; RRMS: relapsing remitting multiple sclerosis; SPMS: secondary progressive multiple sclerosis 


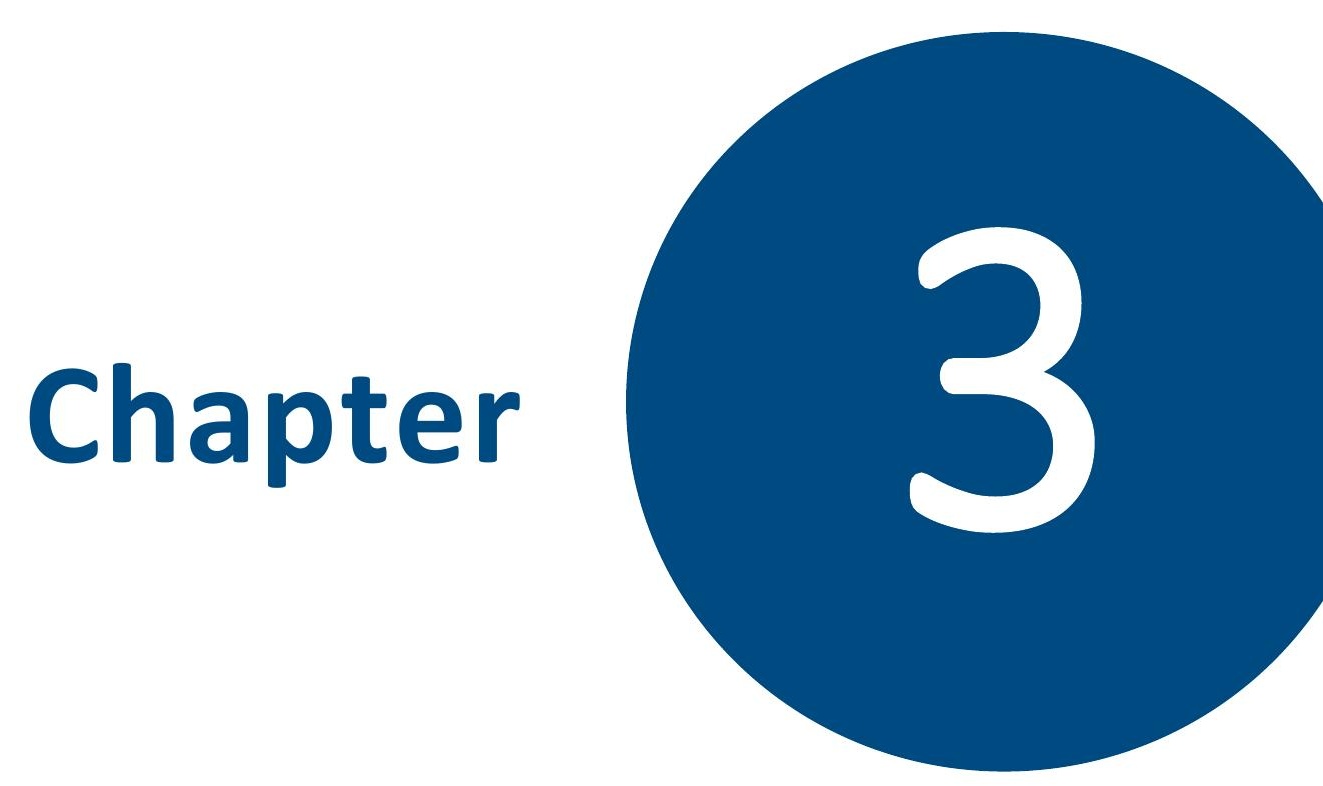

\section{Vitamin D and conversion to progressive disease in patients with multiple sclerosis}

Published as: A low vitamin D status at diagnosis is associated with an early conversion to secondary progressive multiple sclerosis

Muris, A.H., Rolf L., Broen K., Hupperts R., Damoiseaux, J., Smolders J. J Steroid Biochem Mol Biol. 2015 Nov 17. Pii: S0960-0760(15)30136-9. 


\begin{abstract}
Low circulating 25-hydroxyvitamin D (25(OH)D) levels have been associated with an increased risk of relapses in relapsing remitting multiple sclerosis (RRMS), but an association with disability progression is uncertain. Lower 25(OH)D levels are found in secondary progressive MS (SPMS) when compared to RRMS. We hypothesized that a poor vitamin D status in RRMS is associated with an increased risk of conversion to SPMS.
\end{abstract}

In a retrospective longitudinal study we measured 25(OH)D levels at the start of a 3year follow-up, and analysed whether these levels predict the risk of RRMS to SPMS conversion.

In 338 RRMS patients, vitamin D status did not predict the 3-year risk of conversion to SPMS ( $n=51 ;$ OR 0.970; $p=0.65$ ). However, in diagnostic blood samples of SPMS patients with a relatively short RRMS duration $(n=19)$ 25(OH)D levels were significantly lower (38 nmol/L; Q1-Q3: 24-50) than in diagnostic samples of matched RRMS patients with no progression to SPMS ( $\mathrm{n}=38 ; 55 \mathrm{nmol} / \mathrm{L} ; \mathrm{Q1}-\mathrm{Q} 3: 40-70 ; \mathrm{p}<0.01)$.

These data indicate an association between a low vitamin D status at the start of RRMS and the early conversion to SPMS. Therefore, time to SPMS conversion is of interest as clinical measure in (follow-up of) clinical vitamin D supplementation studies. 


\section{Introduction}

One of the major concerns amongst patients with multiple sclerosis (MS) and their clinicians is progressive neurological disability, which is inevitable in most cases. Neurological deficits in this inflammatory disorder are the result of demyelinating lesions in the central nervous system. In its most prevalent phenotypic entity MS starts with a relapsing remitting (RR) phase. [19] In this phase sub-acute episodes of neurological deterioration, called relapses, usually recover. However, in the majority of RRMS patients, recovery between relapses will become incomplete after several years, and there will be progressive worsening between relapses or without any new relapses. $[228,229]$ In either case, patients have entered the secondary progressive (SP)MS phase. $[211,228]$ The pace at which disability accumulates in the RRMS phase is variable, and some patients never convert to SPMS during their disease course. [229-231] The use of disease modifying treatments (DMT) prevents relapses and postpones the accumulation of disability in the RRMS phase. [232] However, currently registered DMTs still cannot protect against disability progression in the SPMS phase. [233]

Likewise, vitamin $D$ status is predominantly associated with the disease course in the RR phase of MS. A high vitamin D status has been associated with a lower risk of relapses in RRMS [74], which may be true particularly for patients at the start of their disease or for younger patients $[103,206]$. Although negative correlations between disability and vitamin D status have been described in MS cohorts [73, 103], an effect of vitamin D status on disability progression was not found in longitudinal $[234,235]$ and cross-sectional data [73]. Only at the start of disease, in clinically isolated syndrome (CIS) patients, a higher vitamin D status predicts less disability progression. [206] Interestingly, we observed lower 25(OH)D levels in SPMS when compared to RRMS patients [103], which could indicate an increased vulnerability to develop SPMS in RRMS patients with a poor vitamin D status.

A delay in the onset of the SP phase in RRMS patients is highly relevant, since this could delay the accumulation of disability and prolong the window of opportunity for treatment with DMTs. Therefore, we assessed whether the vitamin D status in RRMS patients is associated with the time to conversion to SPMS.

\section{Material and methods}

\section{Patients}

In this retrospective longitudinal study subjects with MS were selected from a longitudinal vitamin D study cohort of the Academic MS Centre Limburg (Zuyderland Medical Centre, Sittard, the Netherlands), which consists of all patients with MS according to the original or 2005 revised McDonald criteria [22, 24] who visited the centre in the period 2005 to 2013 and of whom vitamin D data were available. To assess whether the vitamin D status in established RRMS predicts a 3-year risk of conversion to SPMS, all RRMS patients with vitamin D assessment at the start of a 3-year follow-up were included. To assess whether vitamin D status at diagnosis is associated with conversion to SPMS, index subjects with SPMS were 
selected, when both 25 -hydroxyvitamin $\mathrm{D}(25(\mathrm{OH}) \mathrm{D})$ levels or blood samples at diagnosis $( \pm$ 1 year) and reported dates of conversion to SPMS were available. Those SPMS patients were matched in a 1:2 ratio with RRMS control patients, based on their sex, year of birth and year of MS diagnosis. Therefore, compared to the SPMS index patients, the RRMS control patients by definition had a longer RRMS duration, with also several years of progression-free followup. By matching for year of birth and year of diagnosis, SPMS index and RRMS control patients were diagnosed and treated with MS therapies within the same timeframe.

\section{Endpoint definition and 25(OH)D measurement}

No clear clinical criteria are available to define the transition point from RRMS to SPMS, and the diagnosis of SPMS is mostly made retrospectively. [228] Nevertheless, clinicians have to determine this point to make treatment decisions in daily practice. In this study, the transition point was determined by the treating physician $(\mathrm{RH})$, defined as the visit after which the RRMS patient starts to accumulate sustained disability. To describe disability in MS the most widely accepted clinimetrical scale is the expanded disability status scale (EDSS). [209] SPMS was defined as an increased EDSS-score independent of relapses, but not necessarily without relapses. This definition has also been used in other studies. [231]

Serum 25(OH)D levels were measured to assess vitamin D status. The samples of the 3-year follow-up sub-study were analysed as part of routine patient care. Before 2008, first a radioimmunoassay (RIA; Immunodiagnostics Systems, Boldon, UK), and later a chemiluminescence immunoassay (CLIA; Nichols Institute Diagnostics, California, USA) were used for 25(OH)D measurement, due to changed procedures in the clinical setting. For later samples, $25(\mathrm{OH}) \mathrm{D}$ levels were determined with a CLIA (LIAISON ${ }^{\circledR} 25 \mathrm{OH}$ Vitamin D TOTAL, Diasorin, Saluggia, Italy).

For the diagnostic samples, 25(OH)D levels at diagnosis ( \pm 1 year) were available from routine patient care for 19 index and control patients. Seven of them were measured before 2008 (RIA or CLIA (Nichols Institute Diagnostics)). For the remaining 38 patients, stored $\left(-80^{\circ} \mathrm{C}\right.$ ) blood samples ( \pm 1 year from diagnosis) were collected and $25(\mathrm{OH}) \mathrm{D}$ levels were measured using the CLIA (LIAISON ${ }^{\circledR} 25 \mathrm{OH}$ Vitamin D TOTAL, Diasorin).

To correct for seasonal variation the $25(\mathrm{OH}) \mathrm{D}$ levels were deseasonalised, according to the sinusoidal model described by van der Mei et al. [73]. Hereto the total of all consecutive $25(\mathrm{OH}) \mathrm{D}$ levels from all patients of the longitudinal vitamin $\mathrm{D}$ cohort were used to model seasonal variation. The adjusted $25(\mathrm{OH}) \mathrm{D}$ value was calculated by applying the deviation of an individual from the population mean at a time-point measured $(\mathrm{Tm})$, on the population mean at $\mathrm{T}=0$.

\section{Statistics}

All statistical analyses were performed using SPSS software (SPSS Inc., version 20.0, Chicago, USA). Differences in 25(OH)D levels between RRMS and SPMS patients were analysed with a Mann-Whitney $U$ test. A linear regression model was used to assess which patient characteristics were associated with the vitamin D status in SPMS. The factors entered in this model, 
which were linked to the vitamin D assessment date, were sex, age, EDSS score, relapse rate in the previous 3 years, disease duration, and RRMS duration.

To assess whether the vitamin D status contributes to the risk of conversion to SPMS during a 3-year follow-up (yes/no), logistic regression models were used. Several covariates, linked to the vitamin $D$ assessment date, were included in these models: sex, age, EDSS score, disease duration, relapse rate in the previous 3 years, and the deseasonalised 25(OH)D level. Also potential relevant interactions between these covariates were included in the analysis and omitted from the analysis when not statistically relevant using stepwise backward modelling.

To compare 25(OH)D levels at MS diagnosis between SPMS index and the matched RRMS control patients, a Mann-Whitney $U$ test was used. Group differences after matching were analysed by chi-square tests for binomial data, or student's T tests or Mann-Whitney $U$ tests for continuous data. Year of birth and year of MS diagnosis were compared by using the age and disease duration calculated at the day of statistical analysis, respectively. Odds ratios were calculated using simple logistic regression.

A p-value $<0.05$ was considered statistically significant.

\section{Results}

\section{5(OH)D levels are lower in SPMS than in RRMS patients}

First, we confirmed that SPMS patients have lower 25(OH)D levels than RRMS patients. In our longitudinal vitamin D study cohort, the median $25(\mathrm{OH}) \mathrm{D}$ level of SPMS patients $(n=118)$ was $43 \mathrm{nmol} / \mathrm{L}$ (Q1-Q3: 29-60), which was lower than the median 25(OH)D levels of RRMS patients ( $n=338 ; 58 \mathrm{nmol} / \mathrm{L} ; \mathrm{Q} 1-\mathrm{Q} 3$ : 39-77) $(\mathrm{p}=<0.01)$. Patient characteristics are shown in Table 1. Then, we explored which factors could be related to the lower vitamin D status in SPMS patients, and found that RRMS duration was positively associated with vitamin $D$ status. The fit of the model, with 25(OH)D level as dependent variable, improved significantly by adding the combined effect of RRMS duration and RRMS duration ${ }^{2}(p=0.01)$.

Table 1. Patient characteristics of the RRMS and SPMS patients of the longitudinal vitamin D cohort.

\begin{tabular}{lll} 
& RRMS (n=338) & SPMS (n=118) \\
\hline M/F ratio ( $)$ & $73 / 265$ & $47 / 71$ \\
Age (years) (mean ( \pm SD)) & $42.0( \pm 10.0)$ & $53.8( \pm 10.1)$ \\
Disease duration from diagnosis (years) (mean ( $\pm S D))$ & $5.0( \pm 6.3)$ & $13.9( \pm 7.8)$ \\
Relapses in previous 3 years (n) (median (Q1-Q3)) & $1(0-2)$ & $0(0-1)$ \\
EDSS-score (median (Q1-Q3)) & $2.0(1.5-4.0)$ & $6.5(5.9-7.0)$ \\
\hline
\end{tabular}

RRMS: relapsing remitting multiple sclerosis, SPMS: secondary progressive multiple sclerosis, M: male, F: female, EDSS: expanded disability status scale, Q1-Q3: $25^{\text {th }}-75^{\text {th }}$ quartile 
Table 2. Effect of vitamin D status on the SPMS conversion risk.

\begin{tabular}{|lllll} 
& B & OR & 95\% CI OR & p-value \\
\hline Simple model & -0.105 & 0.900 & $0.804-1.008$ & 0.07 \\
Model 1 & -0.048 & 0.953 & $0.842-1.078$ & 0.44 \\
Model 2 & -0.034 & 0.966 & $0.852-1.096$ & 0.59 \\
Model 3 & -0.030 & 0.970 & $0.853-1.104$ & 0.65 \\
\hline
\end{tabular}

Effect of deseasonalised 25(OH)D (per $10 \mathrm{nmol} / \mathrm{L}$ ) on conversion to SPMS (yes/no) during a 3-year follow-up in RRMS patients $(n=338)$ in a logistic regression model. 51 patients converted from RRMS to SPMS.

Simple model includes only deseasonalised 25(OH)D level as a covariate.

Model 1 includes deseasonalised 25(OH)D level, age, disease duration, sex, EDSS and relapse rate in the previous 3 years as covariates

Model 2 includes deseasonalised 25(OH)D level, age, disease duration, sex, EDSS, relapse rate in the previous 3 years, and relapse rate in the previous 3 years $^{2}$ (centred) as covariates.

Model 3 includes deseasonalised 25(OH)D level, age, disease duration, sex, EDSS, relapse rate in the previous 3 years, and relapse rate in the previous 3 years $^{2}$ (centred), disease duration ${ }^{*}$ sex and age*relapse rate in the previous 3 years as covariates.

* significant effect of deseasonalised baseline 25(OH)D at $p<0.05 ; \mathrm{CI}$ : confidence interval; OR: odds ratio

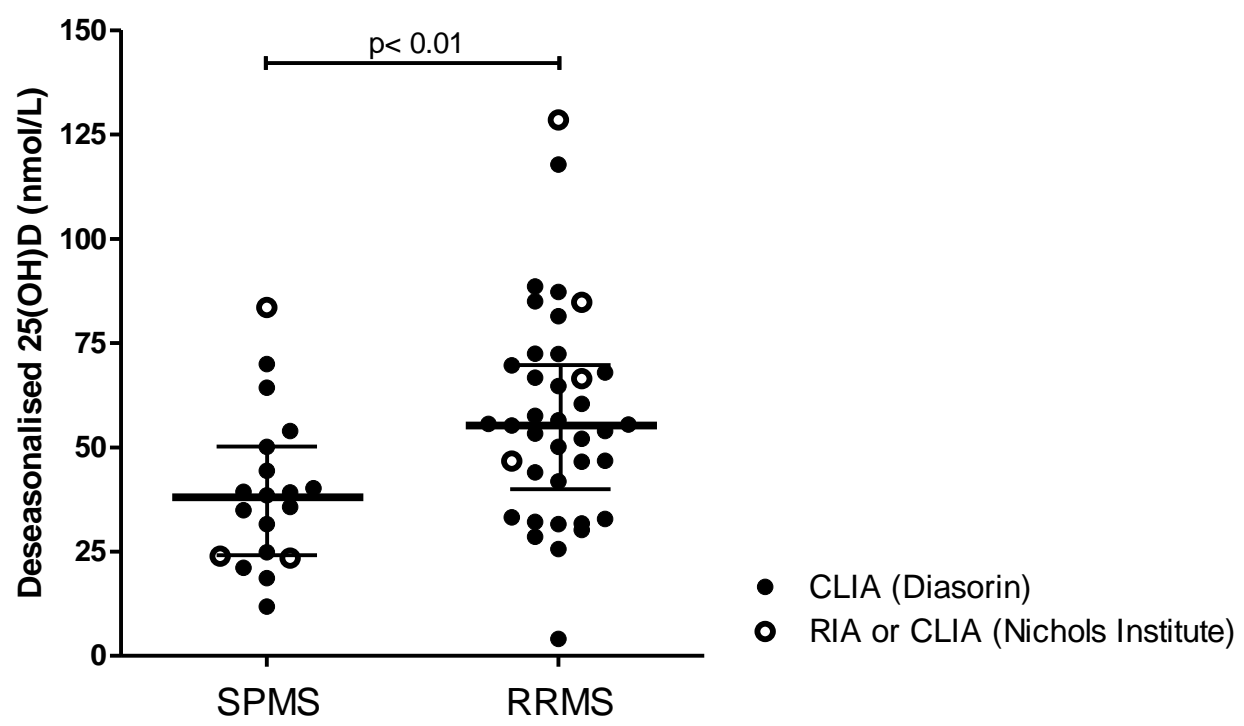

Figure 1. Deseasonalised 25(OH)D levels of SPMS and RRMS patients at MS diagnosis.

Bold lines indicate medians, and the fine lines indicate interquartile ranges.

25(OH)D: 25-hydroxyvitamin D; SPMS: secondary progressive multiple sclerosis; RRMS: relapsing remitting multiple sclerosis; CLIA: chemiluminescence immunoassay; RIA: radioimmunoassay 
Table 3. Patient characteristics of the SPMS index patients and the matched RRMS control patients.

\begin{tabular}{llll} 
& SPMS (n=19) & RRMS (n=38) & p-value \\
\hline M/F ratio (n) & $6 / 13$ & $12 / 26$ & 1.00 \\
Age (years) (mean ( $\pm S D)$ ) & $55.1( \pm 8.4)$ & $53.1( \pm 7.4)$ & 0.38 \\
$\begin{array}{l}\text { Disease duration from diagnosis (years) } \\
\text { (median (Q1-Q3)) }\end{array}$ & $9.7(6.6-12.0)$ & $7.7(6.3-10.0)$ & 0.08 \\
RRMS duration (years) (median (Q1-Q3)) & $3.5(1.0-5.7)$ & $7.7(6.3-10.0)$ & $<0.01^{*}$ \\
Age at diagnosis (years) (mean ( \pm SD)) & $45.4( \pm 8.7)$ & $45.3( \pm 8.2)$ & 0.96 \\
\hline EDSS-score (median (Q1-Q3)) & $3.0(2-4)$ & $2.0(1.5-2.8)$ & 0.08 \\
\hline
\end{tabular}

* significant independent variable at $p<0.05 ;$ SD: standard deviation; SPMS: secondary progressive multiple sclerosis, RRMS: relapsing remitting multiple sclerosis, M: male, F: female, Q1-Q3: $25^{\text {th }}-75^{\text {th }}$ quartile.

\section{5(OH)D levels in RRMS do not predict the 3-year risk of conversion to SPMS}

To assess whether 25(OH)D levels in the RRMS phase predict the risk of conversion to SPMS, we longitudinally analysed 338 RRMS patients. The cohort was characterized as indicated in Table 1. During the 3-year follow-up, 51 (15\%) patients converted to SPMS. Vitamin D status was not a significant predictor of risk of conversion to SPMS, neither in the simple nor in the more advanced regression models (Table 2). Factors that did predict the risk to SPMS conversion were a higher age (model 2: OR 1.054; $p=0.01$ ) and EDSS score (model 2: OR 1.828; $\mathrm{p}<0.01)$ at vitamin $\mathrm{D}$ assessment.

\section{Diagnostic 25(OH)D levels are lower in SPMS patients with a short RRMS duration than in matched RRMS patients}

We did not find an association between vitamin $\mathrm{D}$ status and the 3-year risk of conversion to SPMS, but 25(OH)D levels very early in the disease course of RRMS may be more relevant to predict conversion to SPMS. To investigate this hypothesis, 19 SPMS index patients with a relatively short RRMS duration were matched with 38 RRMS control patients who had not (yet) converted to SPMS. After matching, no statistically significant differences were found in the distribution of sex, age, disease duration, age and EDSS at MS diagnosis (Table 3). The median disease duration of RRMS patients (7.7; Q1-Q3: 6.3-10.0) was significantly longer than the RRMS duration of the SPMS patients (3.5; Q1-Q3: 1.0-5.7; $p<0.01$ ). The SPMS patients had significantly lower 25(OH)D levels (38 nmol/L; Q1-Q3: 24-50) than the RRMS patients (55 nmol/L; Q1-Q3: 40-70; $\mathrm{p}<0.01$ ) (Figure 1). Alternatively expressed, MS patients within the lowest tertile of diagnostic $25(\mathrm{OH}) \mathrm{D}$ levels $(4-36 \mathrm{nmol} / \mathrm{L})$ had a 5.9 -times $(95 \% \mathrm{CI}$ : 1.3-27.3) increased risk of being in the SPMS cohort, when compared to the highest tertile $(58-129 \mathrm{nmol} / \mathrm{L})(\mathrm{p}=0.02)$. 


\section{Discussion}

In our retrospective, longitudinal sub-study, we confirmed the presence of lower $25(\mathrm{OH}) \mathrm{D}$ levels in SPMS when compared to RRMS patients. We found no effect of deseasonalised 25(OH)D levels in established RRMS on the risk of conversion to SPMS within a 3-year follow-up. In our second sub-study, however, vitamin D status at the time of MS diagnosis was significantly lower in SPMS patients, with a relatively short RRMS duration, compared to RRMS patients who had not converted to SPMS (yet). These data suggest that a low vitamin D status at the start of the RRMS disease course is a risk factor for early conversion to SPMS. In our current longitudinal study a higher age and a higher EDSS-score were positive predictors of conversion to SPMS during a 3-year follow-up. These are factors known to be associated with progressive disease in natural history studies. [230, 231, 236] Vitamin D status, however, was not associated with short-term conversion to SPMS. This is in line with the pathophysiological concept that neurodegeneration in SPMS is a process which commences many years before the actual SPMS diagnosis. [19] Therefore, an effect of vitamin D status on SPMS conversion might not be expected in a later stage of RRMS, where this neurodegeneration process is already ongoing. Alternatively, we cannot exclude that the sample size is too small, or the follow-up duration too short to detect an effect.

Interestingly, diagnostic 25(OH)D levels were lower in the RRMS patients with an early conversion to SPMS, compared to those of RRMS patients remaining longer in an RRMS phenotype. This is consistent with accumulating data pointing to the very early RRMS/CIS phase in which a relationship between vitamin $\mathrm{D}$ status and clinical disease activity is most eminent. In a cross-sectional design, we found higher $25(\mathrm{OH}) \mathrm{D}$ levels in relapse-free patients with a disease duration shorter than 5 years. [103] In a longitudinal analysis, higher 25(OH)D levels predicted a reduced risk of relapses only in the younger RRMS patients in our cohort. [235] In the study by Simpson et al., higher 25(OH)D levels were associated with a lower hazard of relapses in an RRMS cohort with a mean disease duration of 6.8 years. [74] In the study by Ascherio et al., reporting a faster rate of EDSS progression in subjects with low 25(OH)D levels, only CIS patients were included. [206] These studies and the present data point to the early phase of MS, which may provide a window of opportunity for intervention studies with vitamin D. This is in accordance with established MS therapies. For example, in CIS patients, a long-term effect of interferon-beta therapy on disease progression was found when treatment was instantly initiated. [237]

Regarding the diagnostic $25(\mathrm{OH}) \mathrm{D}$ levels found in these SPMS index patients, it is noticeable that these were markedly lower than we reported earlier in our RRMS population. [103] This finding is in line with the generally higher $25(\mathrm{OH}) \mathrm{D}$ levels reported in other RRMS $[73,74]$ or CIS populations [206]. Furthermore, the SPMS index patients in our diagnostic sample cohort had a very short RRMS duration, with the 75th percentile at 5.7 years; it is estimated that only $10 \%$ of the RRMS patients convert to SPMS within the first 5 years of disease. [229] It seems that SPMS patients with a very short RRMS duration represent a sub-group of MS patients with lower $25(\mathrm{OH})$ levels at diagnosis. Strikingly, Ascherio et al. argue that CIS pa- 
tients with such low 25(OH)D levels have more cerebral lesion accumulation, more brain loss (atrophy) and a higher annual EDSS increase over time. [206] These observations fit the idea of a shorter progression-free RRMS duration in case of low 25(OH)D levels at the start of disease. This view is supported by experimental data. Zastepa et al. observed that SPMS patients with a short RRMS phase have an increased gene expression of the vitamin $D$ receptor (VDR) in naïve $\mathrm{CD}^{+} \mathrm{T}$ (helper) cells [238], which may be a correlate of insufficient exposure to vitamin D. For interpretation of our results, however, we must take into account the rather small sample size. Furthermore, we noticed a trend towards higher EDSS-scores at MS diagnosis in the SPMS index patients. Therefore, reversed causation cannot be ruled out. However, since EDSS-scores at diagnosis were probably influenced by relapses, it might be as well that the SPMS index patients experienced more severe and/or more multifocal relapses at time of diagnosis compared to the RRMS control patients.

Altogether, our data suggest that low 25(OH)D levels at the diagnosis of RRMS may be associated with an early conversion to SPMS. Whether the associations between vitamin D and MS activity at MS onset are driven by a disease modulating effect of vitamin D on MS should become eminent from clinical supplementation trials. Time to SPMS conversion can be an interesting clinical measure for long-term follow-up in these trials. 



\section{Chapter}

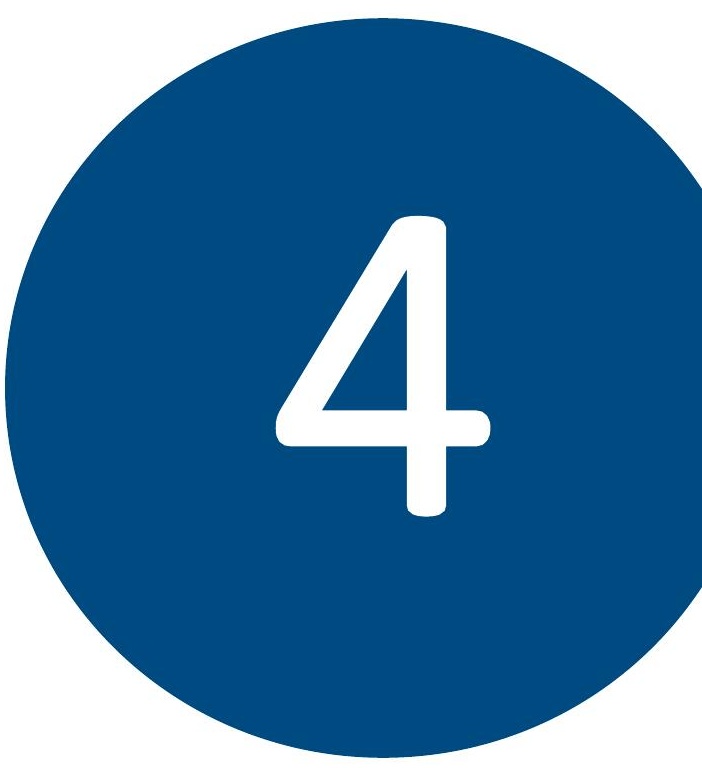

\section{Regulation of encephalitogenic GM-CSF producing $T$ cells by vitamin $D$ and regulatory $T$ cells in multiple sclerosis}

Published as: GM-CSF production by $\mathrm{CD}^{+} \mathrm{T}$ cells in MS patients: regulation by regulatory $\mathrm{T}$ cells and vitamin D;

Peelen E., Muris A.H., Damoiseaux J., Knippenberg S., Broen K., Smolders J., Cohen Tervaert J.W., Hupperts R., Thewissen M.; J Neuroimmunol. 2015 


\section{Abstract}

Data from animal models of multiple sclerosis suggest that $\mathrm{GM}-\mathrm{CSF}^{+} \mathrm{CD}^{+} \mathrm{T}$ cells are pathogenic cells. Therefore, GM-CSF production by $\mathrm{CD}^{+}{ }^{+} \mathrm{T}$ cells of MS patients and their susceptibility to regulatory mechanisms were investigated.

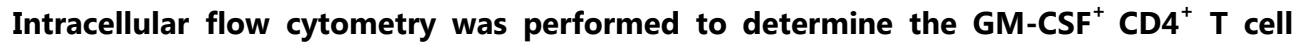
fraction in PBMC and CSF of MS patients and controls. The effect of regulatory $T$ cells (Treg) on GM-CSF production by $\mathrm{CD}^{+} \mathrm{T}$ cells was studied in MS patients using a pro-

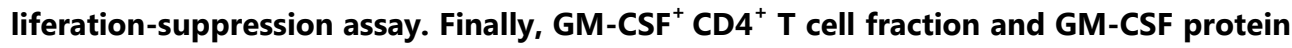
levels in supernatant were assessed in anti-CD3-stimulated $\mathrm{CD}^{+} \mathrm{T}$ cell cultures derived from healthy controls and MS patients, in the presence or absence of the active vitamin D metabolite calcitriol.

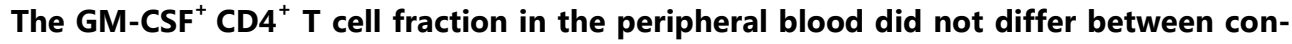
trols and MS patients. This $\mathrm{T}$ cell population could also be detected in the CSF of both subjects with MS as well as subjects with another diagnosis. In the CSF, it comprised a significant fraction of the $\mathrm{T}$ cell population. Upon in vitro stimulation of PBMC with

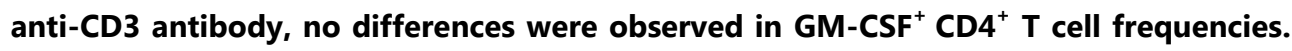
GM-CSF secretion was susceptible to regulation by Tregs and vitamin D. Suppression of GM-CSF secretion by vitamin D was reduced in MS patients.

Our study showed no elevation in $\mathrm{GM}^{-\mathrm{CSF}^{+} \mathrm{CD}^{+}} \mathrm{T}^{\mathrm{T}}$ cell fractions in MS patients compared to controls. Furthermore, GM-CSF secretion was prone to regulation by Tregs and vitamin $D$, the latter being less effective in MS patients. 


\section{Introduction}

Multiple sclerosis (MS) is an inflammatory disease of the central nervous system of autoimmune origin, in which auto-reactive T cells are critically involved. For many years, MS was regarded to be a T helper 1 (Th1) mediated disease. More recently, Th17 cells are believed to be the main pathogenic T cells in MS. In the animal model of MS, both Th1 and Th17 cells are able to adoptively transfer experimental auto-immune encephalomyelitis (EAE). However, knockout models of IFN- $\gamma$ and IL-17A, i.e. Th1 and Th17 cell signature cytokines, are still susceptible to EAE. [38, 239-241] Interestingly, mice with a knockout of either the IL-23 subunit (p19 or p40) have been shown to be protected from EAE development. [242, 243] This cytokine is not only important in the development of pathogenic Th17 cells [244, 245], but also in inducing production of granulocyte macrophage colony-stimulating factor (GM-CSF) by $T$ cells [244].

GM-CSF is a hematopoietic growth factor that is produced by many cell types, including $T$ cells. This cytokine has been described to promote the activation and differentiation of antigen presenting cells and increase the survival of monocytes, macrophages, neutrophils, eosinophils and basophils. [246] It exerts pro-inflammatory functions by increasing antigen presentation and release of inflammatory cytokines. Studies in EAE have demonstrated a critical role for GM-CSF in the disease process, since $\mathrm{GM}^{-\mathrm{CSF}^{-/-}}$mice were resistant to $\mathrm{EAE}$ development. [38, 247] Furthermore, infusion of anti-GM-CSF antibodies before disease onset could prevent EAE induction. Also, complete recovery from EAE was demonstrated when mice were treated with an anti-GM-CSF antibody after onset of the disease. [247] GMCSF marks a highly pathogenic Th cell population. In contrast to any other known helper $T$ cell cytokine, GM-CSF positive $\mathrm{CD}^{+}{ }^{+} \mathrm{T}$ cells exert a non-redundant function in neuro-autoimmune pathogenicity in vivo. $[38,248]$ From these studies it can be concluded that GM-CSF producing $T$ cells have strong encephalitogenic capacity in EAE.

Regulation of potentially pathogenic T cells is important and in healthy individuals this is effectively achieved. One of the most important regulators of effector $T$ cells is the regulatory T cell (Treg), which has been shown functionally defective in MS patients. [115-117] Another important immune regulator is vitamin $D$, which has also been found to inhibit effector $T$ cells directly and enhances numbers and/or function of Tregs (reviewed in [147]). Both Tregs and vitamin $\mathrm{D}$ might be critical for $\mathrm{GM}-\mathrm{CSF}^{+} \mathrm{CD}^{+} \mathrm{T}$ cell regulation.

Because of the potential pathogenic role of GM-CSF producing Th cells, we investigated the expression and secretion of GM-CSF by $\mathrm{CD}^{+}{ }^{+} \mathrm{T}$ cells from MS patients and controls and explored the susceptibility of GM-CSF producing $T$ cells to regulation by Tregs and vitamin D. 
Table 1. Population characteristics.

A. Cross-sectional study cohort; subjects used for the ex vivo experiments (Figure 1B)

\begin{tabular}{|c|c|c|c|c|c|}
\hline & $\mathrm{HC}$ & \multicolumn{4}{|c|}{ RRMS Remission } \\
\hline & \multicolumn{5}{|c|}{ Number (\%)/ median (min-max) } \\
\hline Treatment & - & No & IF $N-\beta$ & GA & Total \\
\hline Number of subjects & 13 & 10 & 5 & 7 & 22 \\
\hline \multicolumn{6}{|l|}{ Gender } \\
\hline Male & $3(23.1)$ & $3(30.0)$ & $2(40.0)$ & $2(28.6)$ & $7(31.8)$ \\
\hline Female & $10(76.9)$ & $7(70.0)$ & $3(60.0)$ & $5(71.4)$ & $15(68.2)$ \\
\hline Age (years) & $\begin{array}{l}29.2 \\
(22.8-56.8)\end{array}$ & $\begin{array}{l}29.9 \\
(23.2-53.3)\end{array}$ & $\begin{array}{l}45.1 \\
(43.7-51.0)\end{array}$ & $\begin{array}{l}40.9 \\
(34.4-49.0)\end{array}$ & $\begin{array}{l}40.7 \\
(23.2-53.3)\end{array}$ \\
\hline Disease duration (yrs) & - & $0.3(0.0-4.2)$ & $0.5(0.4-2.7)$ & $2.6(1.0-4.4)$ & $1.4(0.0-4.4)$ \\
\hline $\begin{array}{l}\text { Relapse rate } \\
\text { (relapse/year) }\end{array}$ & - & $1.0(0.0-2.0)$ & $1.0(1.0-2.0)$ & $0.0(0.0-0.0)$ & $1.0(0.0-2.00)$ \\
\hline EDSS & - & $1.0(0.0-2.0)$ & $2.0(2.0-2.0)$ & $2.0(1.0-6.0)$ & $1.5(0.0-6.0)$ \\
\hline
\end{tabular}

HC: healthy control; RRMS: relapsing remitting multiple sclerosis; EDSS: expanded disability status scale; IFN: interferon; GA: glatiramer acetate; min: minimum; max: maximum; No: no treatment

B. In vitro tests study cohort; subjects used for the in vitro experiments (Figure 284)

\begin{tabular}{|l|ll}
\hline & HC & RRMS Remission \\
\hline $\begin{array}{l}\text { Treatment } \\
\text { Number of subjects }\end{array}$ & Number (\%)/median (min-max) & No \\
Gender & 9 & 8 \\
$\quad$ Male & $3(33.3)$ & \\
$\quad$ Female & $6(66.7)$ & $3(37.5)$ \\
Age (years) & $29.8(22.8-56.8)$ & $5(62.5)$ \\
\hline $\begin{array}{l}\text { Disease duration (years) } \\
\text { Relapse rate (relapse/year) }\end{array}$ & - & $30.0(23.5-53.3)$ \\
EDSS & - & $0.0(0.0-4.2)$ \\
\hline
\end{tabular}

HC: healthy control; RRMS: relapsing remitting multiple sclerosis; EDSS: expanded disability status scale; min: minimum; max: maximum; No: no treatment 


\section{Methods}

\section{Patients and blood samples}

This study consists of 3 parts, each being based on distinct (partially overlapping) patient populations. RRMS patients were recruited from the Academic MS Centre Limburg, Orbis Medical Centre (Sittard, the Netherlands) or the MS Centre of Amphia Hospital (Breda, The Netherlands). Written informed consent was obtained from each study subject for blood sampling (heparinized blood). RRMS was defined by the revised McDonald criteria. [23] All RRMS patients had a disease duration $<6$ years and were in remission $>6$ weeks at the time of inclusion. The study was approved by the regional ethical committee 'Atrium-Orbis-Zuyd' and 'the medical ethical committee of Amphia' of Amphia Hospital.

The ex vivo assays (part 1) were performed on peripheral blood of 22 RRMS patients receiving either no, IFN- $\beta$ or GA treatment, and 13 healthy controls (HC). Population characteristics are given in Table 1A. In addition, for ex vivo assays performed on material derived from paired blood or cerebrospinal fluid (CSF) samples, 8 subjects, suspected of MS at the Academic MS Centre Limburg at Orbis Medical Centre, were included (not included in table 1). Four of these subjects were subsequently diagnosed with MS of which 2 had clinical isolated syndrome, 1 subject had primary progressive MS (PPMS) and 1 subject had clinical definite MS. Three of these MS patients were sampled during remission, and one during active disease. The other four subjects were diagnosed with either a cerebro vascular accident, migraine, systemic lupus erythematosus (SLE) or two-sided opticopathy and were classified as having 'other diagnosis'.

For in vitro assays (part 2) to assess GM-CSF expression by T helper cells after stimulation and the effect of calcitriol on this, 8 treatment naïve RRMS patients and $9 \mathrm{HC}$ were included. Population characteristics of HC and RRMS patients are given in Table 1B.

To assess the effect of Tregs on GM-CSF production by $C D 4^{+} \mathrm{T}$ cells in MS patients (part 3), baseline culture supernatants obtained from functional Treg assays performed in a previous study were available. [79] Culture supernatants of 13 RRMS patients in remission and on IFN$\beta$ therapy were included in the current study.

\section{Cell isolation}

Peripheral blood mononuclear cells (PBMC) were isolated using Ficoll (Histopaque; SigmaAldrich, Zwijndrecht, the Netherlands) density gradient centrifugation and cells within the CSF were collected by centrifugation of the CSF at $4^{\circ} \mathrm{C}$. In a series of in vitro experiments, purified $\mathrm{CD}^{+} \mathrm{T}$ cells were used. These $\mathrm{CD} 4^{+} \mathrm{T}$ cells were isolated from heparinized peripheral blood via negative selection using RosetteSep (STEMCELL technologies, Grenoble, France) followed by a Ficoll density gradient centrifugation step. Purity of the isolated $\mathrm{CD}^{+}$cells within the T cell population usually is between $97.8 \%-99.4 \%$. 


\section{Intracellular cytokine detection}

The $\mathrm{CD}^{+}{ }^{+} \mathrm{T}$ cell cytokine profile in peripheral blood and CSF was determined by flow cytometry by assessing the expression of cytokines within the $\mathrm{CD} 3^{+} \mathrm{CD} 8^{-}$lymphocytes, i.e. $\mathrm{CD} 4^{+}$ T cells, as described before. [79] Shortly, cells were stimulated for 4 or $5 \mathrm{~h}$ with $1 \mu \mathrm{g} / \mathrm{mL}$ ionomycin and $50 \mathrm{ng} / \mathrm{mL}$ PMA (both from Sigma-Aldrich) in the presence of monensin (BD Biosciences, Breda, the Netherlands) to block cytokine secretion. Surface staining was performed with anti-CD3-Horizon V450 and anti-CD8-APC-H7 (both BD Biosciences), and antiGM-CSF-PE (Biolegend, Uithoorn, the Netherlands) was used for the intracellular staining.

\section{Regulation by Tregs and vitamin D}

All cells were cultured in RPMI medium (Gibco Invitrogen, Breda, the Netherlands) supplemented with $10 \%$ foetal bovine serum (Greiner Bio-One, Alphen aan de Rijn, the Netherlands), $1 \%$ non-essential amino acids (Gibco Invitrogen), $1 \mathrm{mM}$ sodium pyruvate (Gibco Invitrogen) and $2 \%$ penicillin-streptomycin (Gibco Invitrogen).

To evaluate the ability of FACS-sorted Tregs to suppress GM-CSF secretion by $\mathrm{CD}^{+}{ }^{+} \mathrm{T}$ cells, culture supernatants from a previously performed Treg suppression assay [79] were analysed for GM-CSF protein content. Shortly, Treg (CD25 high CD127 CD4 ${ }^{+}$T cells) and Tresp (CD25 $\mathrm{CD}^{+} \mathrm{T}$ cells) were activated with anti-CD3 antibody and either cultured alone or in a $1: 1$ coculture. After 4-5 days, culture supernatants were harvested and stored at $-20^{\circ} \mathrm{C}$ until analysis.

To test the effect of calcitriol $\left(1,25(\mathrm{OH})_{2} \mathrm{D}\right.$; the active form of vitamin $\left.\mathrm{D}\right)$ on the expression of GM-CSF by CD4 ${ }^{+} \mathrm{T}$ cells, $1^{*} 10^{5} \mathrm{CD}^{+} \mathrm{T}$ cells were plated in a flat bottom 96 -well plate (Corning Incorporation, Amsterdam, the Netherlands) precoated with anti-CD3 antibody (clone 2G3, kind gift from Biomedical Research Institute (Biomed), Diepenbeek, Belgium). The cells were cultured in the absence or presence of $10^{-8} \mathrm{M}$ calcitriol (Sigma-Aldrich). After 5 days, culture supernatants were collected and stored at $-20^{\circ} \mathrm{C}$ until further analysis. In addition, the $\mathrm{GM}^{-\mathrm{CSF}^{+}}$cell fraction was assessed by intracellular flow cytometry as described above. Brefeldin A ( $5 \mu \mathrm{g} / \mathrm{mL}$; Sigma-Aldrich) was used to block cytokine secretion.

\section{Quantification of GM-CSF protein levels}

GM-CSF protein levels in the culture supernatants were detected by means of cytometric bead array (CBA) flex set technology (BD Biosciences). The beads were prepared as described by the manufacturer. Culture supernatant $(50 \mu \mathrm{L})$ was incubated with GM-CSF capture beads. After $1 \mathrm{~h}$ the GM-CSF PE detection reagent was added and incubated for $2 \mathrm{~h}$. After washing, data acquisition was performed on a FACS Canto II flow cytometer and data were analysed with FACS Diva software.

\section{Statistics}

Statistical analysis was performed with Statistical Package for Social Sciences version 17.0 software (SPSS inc., Chicago IL, USA). Results are given as median with corresponding range (minimum-maximum) for continues variables. The Mann-Whitney $U$ test was performed to assess differences between groups and the Wilcoxon signed-rank test was performed to test 
the difference between paired samples in the absence or presence of calcitriol or Tregs. The percentage inhibition in $\mathrm{T}$ cell frequencies or protein levels by calcitriol was calculated using the formula: (1-[condition with calcitriol]/[condition without calcitriol])x100\%. Spearman correlation was used to test correlations. A p-value $<0.05$ was considered statistically significant.

\section{Results}

\section{GM-CSF positive CD4 ${ }^{+} \mathrm{T}$ cell frequencies in blood and CSF are not different in MS pa- tients}

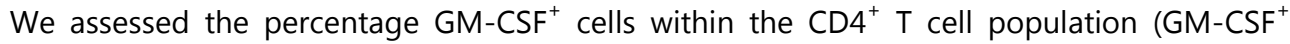
$\mathrm{CD}^{+} \mathrm{T}$ cells $)$ in the peripheral blood of $\mathrm{HC}(\mathrm{n}=13)$ and RRMS patients in disease remission $(n=22)$. For this, PBMC were shortly stimulated ex vivo with PMA and ionomycin in the presence of monensin. Subject characteristics are given in Table 1A. A representative density plot of GM-CSF expression in $\mathrm{CD}^{+} \mathrm{T}$ cells for both $\mathrm{HC}$ and MS patients is shown in Figure 1A. The percentage of this $\mathrm{CD} 4^{+} \mathrm{T}$ cell subset in the peripheral blood was not increased in RRMS patients in remission compared to $\mathrm{HC}$ (7.9\% (2.1-20.1) and 11.2\% (5.7-23.6), p=0.162; Figure 1B). Group sizes are, however, too small to statistically test differences between treated and untreated RRMS patients.

In order to assess whether $\mathrm{GM}-\mathrm{CSF}^{+} \mathrm{CD}^{+} \mathrm{T}$ cells are also present in closer proximity to the CNS, as proof of principle, we examined the relative presence of these cells in paired CSF and PBMC samples of MS patients $(n=4)$ and patients with other diagnoses $(n=4)$ (Figure 1C). Interestingly, these $T$ cell frequencies were 6.8 times (1.5-11.7) higher in CSF versus blood (39.2\% (14.1-59.3) and 6.4\% (3.0-17.8), respectively, $p=0.008)$ for the total cohort. Moreover, the percentage $\mathrm{GM}-\mathrm{CSF}^{+} \mathrm{CD}^{+} \mathrm{T}$ cells in CSF of MS patients did not differ from patients with other diagnosis within this limited sample size $(p=0.386)$.

\section{In vitro GM-CSF expression and secretion by $\mathrm{CD4}^{+} \mathrm{T}$ cells is not elevated in RRMS pa- tients}

To investigate in vitro GM-CSF expression and cytokine secretion, $\mathrm{CD}^{+}{ }^{+} \mathrm{T}$ cells of treatment naïve RRMS patients $(n=8)$ and $H C(n=9)$ were stimulated for 5 days with anti-CD3 antibody. Subject characteristics are given in Table 1B. After 5 days of culture, the percentage GM-CSF ${ }^{+}$ $\mathrm{CD}^{+} \mathrm{T}$ cells was similar for HC and RRMS patients (35.8\% (19.9-49.9) and 32.1\% (16.3-42.7), respectively, $p=0.386$, Figure $2 A$ ). GM-CSF protein levels in the supernatant of stimulated $\mathrm{CD}^{+} \mathrm{T}$ cells were not significantly different between $\mathrm{HC}$ and RRMS patients $(5887 \mathrm{pg} / \mathrm{mL}$ (4816-8861) and $5067 \mathrm{pg} / \mathrm{mL}$ (3618-6937), respectively, $\mathrm{p}=0.083$, Figure 2B). 
A

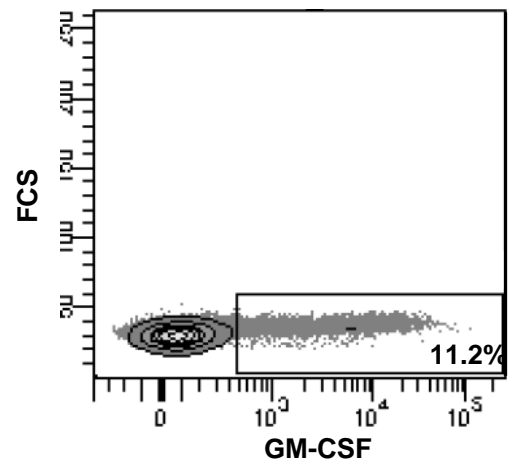

B

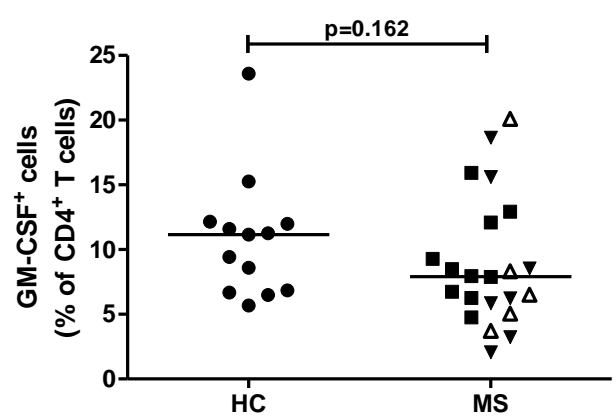

MS

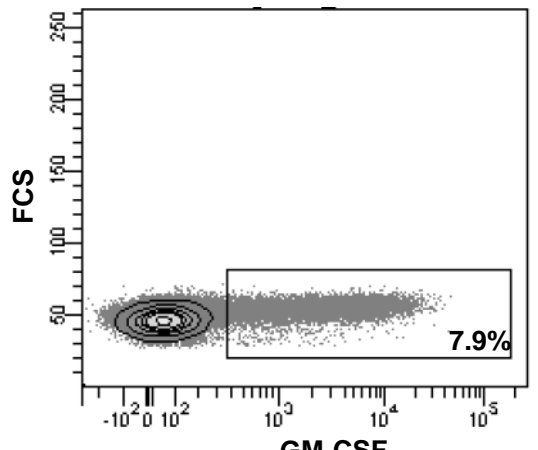

GM-CSF

C

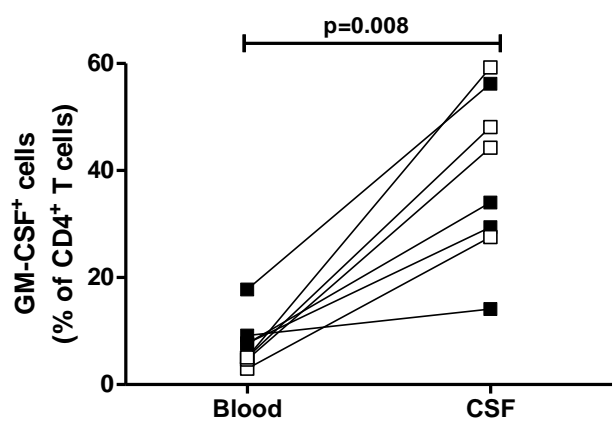

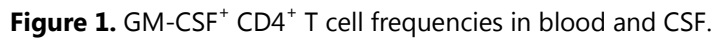

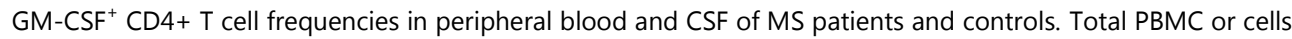
from the CSF were stimulated with PMA and ionomycin for 5 hours in the presence of monensin. A) Density plots of GM-CSF positive cells in $\mathrm{CD}^{+} \mathrm{T}$ cells of a representative RRMS patient (right) and control subject (left). B) GM-CSF $\mathrm{CD}^{+}$T cell frequencies in peripheral blood of $\mathrm{HC}(n=13)$ and RRMS patients $\left.(n=22), C\right) \mathrm{GM}-\mathrm{CSF}^{+}$ $\mathrm{CD}^{+} \mathrm{T}$ cell frequencies in paired CSF and blood samples of subjects with other diagnosis $(n=4)$ and MS patients $(n=4)$. Differences between groups were assessed by the Mann-Whitney $U$ test $(B)$, and differences between paired samples were assessed with the Wilcoxon sign-rank test (C). A p-value $<0.05$ was considered statistically significant. In B) closed squares represent RRMS patients not on immune modulatory therapy; open triangles represent RRMS patients on interferon-beta and inversed closed triangles represent RRMS patients on glatiramer acetate. In C) closed squares represent MS patients, while open squares represent subjects with other diagnosis.

CD: cluster of differentiation; CSF: cerebrospinal fluid; FSC: forward scatter; GM-CSF: granulocyte-macrophagecolony-stimulating factor; HC: healthy control; MS: multiple sclerosis 
A

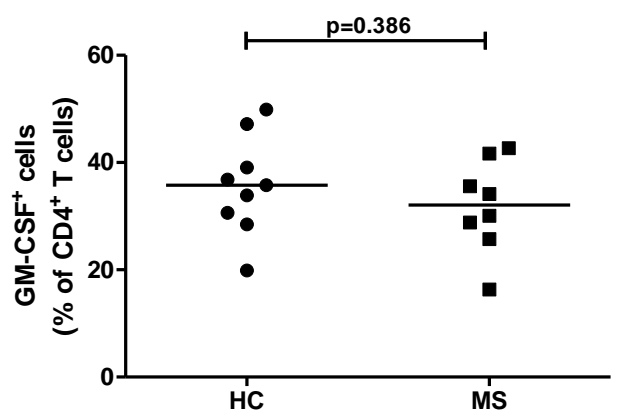

B

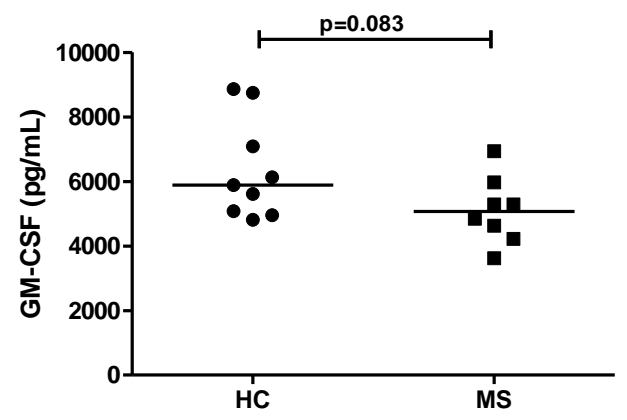

Figure 2. GM-CSF expression and secretion by in vitro cultured $C D 4^{+} \mathrm{T}$ cells.

$\mathrm{CD}^{+} \mathrm{T}$ cells from healthy controls $(\mathrm{HC})$ and treatment-naïve RRMS patients (MS) were stimulated in vitro with plate bound anti-CD3 antibody for 5 days. A) $\mathrm{GM}-\mathrm{CSF}^{+} \mathrm{CD}^{+} \mathrm{T}$ cell frequencies and B) $\mathrm{GM}-\mathrm{CSF}$ protein levels in the culture supernatant of $C D 4^{+} T$ cells in $H C(n=9)$ and MS patients $(n=8)$. Differences between groups were assessed with Mann-Whitney $\mathrm{U}$ test and a $\mathrm{p}$-value $<0.05$ was considered statistically significant.

CD: cluster of differentiation; GM-CSF: granulocyte macrophage colony-stimulating factor; HC: healthy control, MS: multiple sclerosis

\section{Autologous Tregs are able to regulate GM-CSF secretion by $\mathrm{CD}^{+} \mathrm{T}$ cells of RRMS pa- tients}

Tregs have been shown to be able to regulate pathogenic $T$ cell responses and therefore might also be able to suppress GM-CSF secretion by $\mathrm{CD}^{+} \mathrm{T}$ cells. GM-CSF protein levels were determined in the stored culture supernatants of a historically performed functional Treg assay from 13 RRMS patients. [79] Tresp and Treg were either cultured separately or together for 4 to 5 days. Upon stimulation with anti-CD3 antibody, Tresp were found to secrete considerable amounts of GM-CSF (118.2 pg/mL (64.9-224.6)) while Tregs secreted only minimal amounts of GM-CSF (4.7 pg/mL (0.0-25.3), $\mathrm{p}=0.001$, Figure 3 ). In the co- cultures of Tresp and Treg, we found that GM-CSF protein levels were significantly lower (7.4 $\mathrm{pg} / \mathrm{mL}(0.0-20.4))$ as compared to Tresp mono-cultures $(\mathrm{p}=0.001$, Figure 3$)$.

Since Tregs appear to inhibit GM-CSF secretion by $\mathrm{CD}^{+} \mathrm{T}$ cells, there might be an association between Tregs and $\mathrm{GM}-\mathrm{CSF}^{+} \mathrm{CD}^{+} \mathrm{T}$ cell frequencies in vivo. However, our data revealed no association between these two cell subsets in the different cohorts (data not shown). 


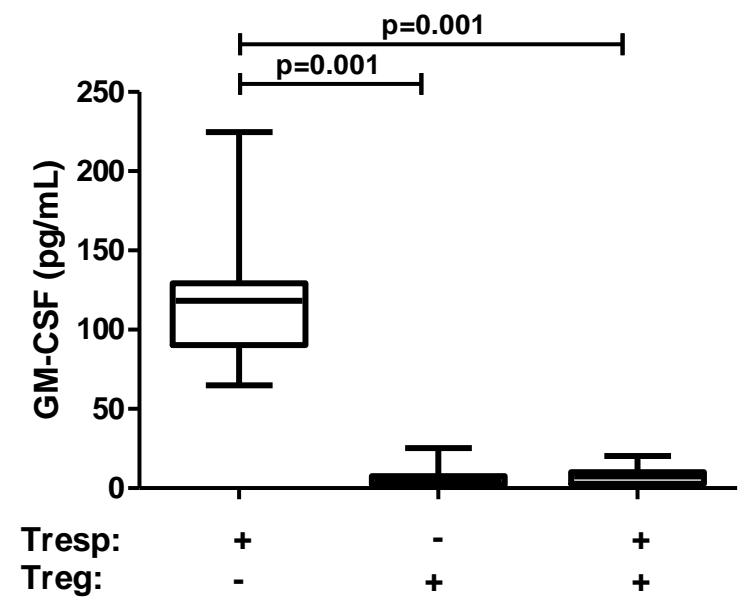

Figure 3. Regulation of GM-CSF producing $\mathrm{CD}^{+} \mathrm{T}$ cells by regulatory $\mathrm{T}$ cells.

GM-CSF protein levels in the culture supernatant of anti-CD3 antibody activated T responder cells (Tresp, CD25 $\mathrm{CD}^{+} \mathrm{T}$ cells), Tregs (CD25 high CD127 CD4 ${ }^{+} \mathrm{T}$ cells) or a 1:1 coculture of Tresp cells and Tregs from 13 RRMS patients on interferon-beta therapy. The boxes represent the $25^{\text {th }}$ and the $75^{\text {th }}$ percentile with the dark horizontal lines inside the boxes representing the median. The whiskers represent the minimum and maximum values. Statistical differences between conditions were tested with the Wilcoxon signed-rank test. A p-value $<0.05$ was considered statistically significant.

CD: cluster of differentiation; GM-CSF: granulocyte macrophage colony-stimulating factor; Treg; regulatory $T$ cell, Tresp: responder T cell

\section{Calcitriol-mediated suppression of GM-CSF secretion by $\mathrm{CD}^{+} \mathrm{T}$ cells is impaired in RRMS patients}

Calcitriol has been described to be a potent immune modulator that can reduce pathogenic $T$ cell responses. Therefore, we assessed the effect of calcitriol on GM-CSF producing $\mathrm{CD}^{+} \mathrm{T}$ cells in vitro. GM-CSF ${ }^{+} \mathrm{CD}^{+} \mathrm{T}$ cell frequencies were equally reduced by calcitriol in $\mathrm{HC}(\mathrm{n}=9)$ and RRMS ( $\mathrm{n}=8$ ) patients (inhibition: $82.7 \%(49.0-91.1)$ and 76.8\% (31.6-87.8), respectively, $\mathrm{p}=0.773$, Figure $4 A-B$ ). Interestingly, the reduction in GM-CSF protein levels in the culture supernatant in the presence of calcitriol was more pronounced in the samples from $\mathrm{HC}$ compared to RRMS patients (inhibition: $80.9 \%$ (63.5-89.8) and $64.9 \%(-15.5-76.8)$, respectively, $p=0.021$, Figure 4C-D). 

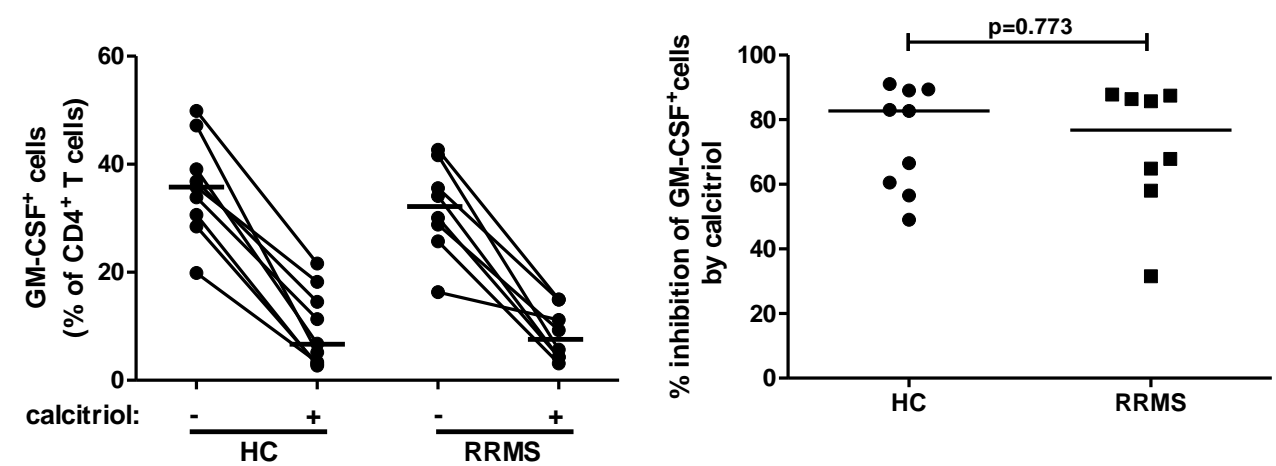

\section{C}

D
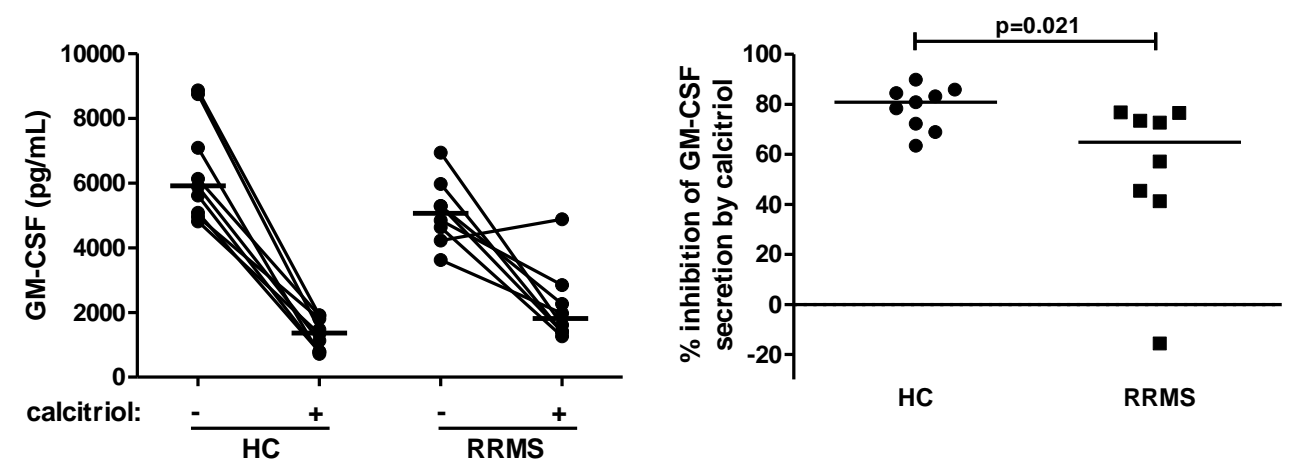

Figure 4. Regulation of GM-CSF expression and secretion of $\mathrm{CD}^{+} \mathrm{T}$ cell by calcitriol.

$\mathrm{CD}^{+} \mathrm{T}$ cells from peripheral blood of healthy controls $(\mathrm{HC}, \mathrm{n}=9)$ and treatment-naïve RRMS patients (RRMS, $\mathrm{n}=8$ ) were cultured in vitro and activated with plate bound anti-CD3 antibody for 5 days in the absence (-) or presence (+) of calcitriol. A) Frequencies of $\mathrm{GM}_{-}-\mathrm{CSF}^{+} \mathrm{CD}^{+} \mathrm{T}$ cells after culture in the absence or presence of calcitriol, B) the percentage of inhibition of $\mathrm{GM}_{-} \mathrm{CSF}^{+} \mathrm{CD}^{+} \mathrm{T}$ cell frequencies by calcitriol relative to the condition without calcitriol, C) GM-CSF protein levels in the culture supernatant of $\mathrm{CD4}^{+} \mathrm{T}$ cells in the absence or presence of calcitriol and D) the percentage inhibition of GM-CSF protein levels by calcitriol relative to the condition without calcitriol. Horizontal bars represent the median. Differences between groups were assessed with Mann-Whitney $\mathrm{U}$ test and a $\mathrm{p}$-value $<0.05$ was considered statistically significant.

CD: cluster of differentiation; GM-CSF: granulocyte macrophage colony-stimulating factor; HC: healthy control; RRMS: relapsing remitting multiple sclerosis 


\section{Discussion}

In the present study, we showed that the frequency of $\mathrm{GM}-\mathrm{CSF}^{+} \mathrm{CD}^{+} \mathrm{T}$ cells in the circulation of RRMS patients in remission is not elevated compared to healthy controls. Interestingly, GM-CSF${ }^{+} \mathrm{CD}^{+} \mathrm{T}$ cells frequencies were enriched in CSF as compared to blood. Furthermore, we showed that Tregs from RRMS patients are capable to strongly reduce GM-CSF secretion by $\mathrm{CD}^{+} \mathrm{T}$ cells. Finally, calcitriol appeared to be able to reduce the fraction of $\mathrm{GM}$ $\mathrm{CSF}^{+} \mathrm{CD4}^{+} \mathrm{T}$ cells in RRMS patients and $\mathrm{HC}$, but suppression of GM-CSF secretion by calcitriol was significantly reduced in RRMS patients compared to $\mathrm{HC}$.

A pathogenic role of GM-CSF has been described in experimental models of arthritis, autoimmune myocarditis and multiple sclerosis. [247, 249, 250] More recently, GM-CSF expressing $\mathrm{CD}^{+}{ }^{+} \mathrm{T}$ cells have been identified as the dominant pathogenic cells in EAE. [38] Expression of GM-CSF mRNA has been shown in CNS tissue of mice 7-8 days after EAE induction. $[248,251]$ Dependent on the study, GM-CSF mRNA was already decreasing at day 15 or 21 after induction. The results from Ponomarev et al. indicate that this GM-CSF was derived from the T cells, since there was only a low expression of GM-CSF in the spinal cord of mice that were induced with EAE by MBP-TCR GM-CSF ${ }^{-}$T cells compared to WT MBP-TCR T cells. [248] Moreover, they showed that mice receiving WT MBP-TCR T cells had 7.8\% GMCSF expressing cells within T cells derived from CNS tissue at the peak of disease (day 14). The exact mechanism of action for $\mathrm{CD}^{+}{ }^{+} \mathrm{T}$ cell derived GM-CSF in EAE is unknown. It is postulated, however, that its effects on the myeloid cell compartment, i.e., microglial activation and/or myeloid cell recruitment to CNS, is of utmost importance. [38, 248] Moreover, GMCSF has been shown to stimulate IL- 6 production by dendritic cells, which is required for Th17 cell differentiation and also appears to be important for survival of activated T cells. [250] In contrast, studies performed in experimental mice models of auto-immune thyroiditis, myasthenia gravis and type I diabetes showed a beneficial effect of GM-CSF infusion. [252] Furthermore, they showed that GM-CSF enhances the proliferation of functional Tregs. [253] However, these studies did not focus on $\mathrm{GM}-\mathrm{CSF}^{+} \mathrm{CD}^{+} \mathrm{T}$ cells. Moreover, it can be expected that GM-CSF expressing $\mathrm{CD} 4^{+} \mathrm{T}$ cells can play variable roles in different target organs.

Since $\mathrm{GM}-\mathrm{CSF}^{+} \mathrm{CD}^{+}$T cells are critical in EAE, we expected an increased frequency of this cell subset in the peripheral blood of RRMS patients compared to HC. Yet, our data did not show an augmented $\mathrm{GM}^{-\mathrm{CSF}^{+}} \mathrm{CD}^{+} \mathrm{T}$ cell population in RRMS patients in remission. Moreover, Noster et al., recently did not find any increase in these cells in MS patients with active disease. [39] In contrast, Hartmann et al. showed that RRMS patients have an increased percentage of $\mathrm{GM}-\mathrm{CSF}^{+} \mathrm{CD}^{+} \mathrm{T}$ cells compared to patients with other non-inflammatory neurological diseases (OND). [254] However, in their validation cohort this difference is only borderline significant. Moreover, they show that medication reduces $\mathrm{GM}-\mathrm{CSF}^{+} \mathrm{CD} 4^{+} \mathrm{T}$ cell frequencies in RRMS patients. Inclusion in our study of patients on first-line MS medication

therefore may have obscured a possible difference. Distinct outcomes may also be related to 
differences in the patient cohorts, the control cohorts, and the work-up of the blood samples.

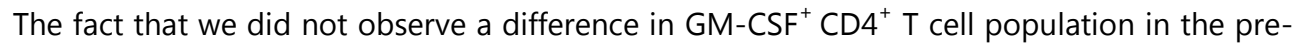
sent study might also be due to the possibility that the $\mathrm{GM}-\mathrm{CSF}^{+} \mathrm{CD}^{+} \mathrm{T}$ cell population represents a heterogeneous pool of pathogenic and non-pathogenic cells similar to the Th17 cell subset. $[244,245]$ This might be in line with the observation that GM-CSF production by $\mathrm{CD}^{+} \mathrm{T}$ cells can only be inhibited by IL-27 in cells that do not co-express IL-17. [255] However, in our current study, also $\mathrm{GM}-\mathrm{CSF}^{+} \mathrm{IL}_{17}{ }^{+} \mathrm{CD}^{+} \mathrm{T}$ cell frequencies did not differ between $\mathrm{HC}$ and RRMS patients (data not shown).

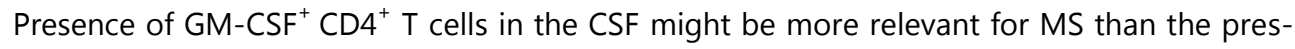
ence of these cells in the peripheral blood. Our results show that $\mathrm{GM}-\mathrm{CSF}^{+} \mathrm{CD}^{+} \mathrm{T}$ cells can indeed be detected in the CSF and there they make up a significant fraction of the $T$ cell population. This observation might indicate that these cells are importantly involved in brain immune pathology. This is in line with the results observed in the study of Noster et al.. Although, earlier studies showed no differences in GM-CSF protein levels in CSF of MS patients in remission compared to controls $[256,257]$, Noster et al. showed that the GM-CSF ${ }^{+}$ $\mathrm{CD}^{+} \mathrm{T}$ cell population is increased in the CSF of MS patients with active disease versus nonMS patients [39]. To make a definitive statement on an enriched fraction of GM-CSF expressing Th cells in the CSF of MS patients, a larger population of MS patients (both during remission and relapse) and controls should be investigated.

Cell frequencies as such do not reflect quantitative cytokine production. Therefore, GM-CSF protein levels were measured in the culture supernatants of activated $\mathrm{CD} 4^{+} \mathrm{T}$ cells. Also, GMCSF protein levels were not elevated in culture supernatant of cells derived from RRMS patients in remission compared to HC. Similar results have been found by Vrethem et al. in

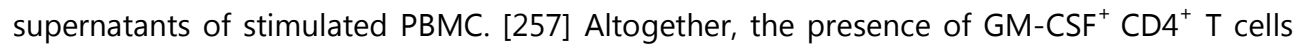
and/or GM-CSF production was not increased in MS patients compared to controls. Future studies employing stimulation with possible auto-antigens, like myelin oligodendrocyte glycoprotein (MOG) and/or myelin basic protein (MBP), might be able to reveal an elevated antigen specific response in MS patients and provide more insight in the relevance of GM$\mathrm{CSF}^{+} \mathrm{CD}^{+} \mathrm{T}$ cells in MS pathogenesis. However, the auto-antigens in MS are still unknown. In addition, $T$ cells reactive to the classical antigens, MOG, MBP and/or proteolipoprotein (PLP), believed to be involved in MS are also observed in healthy individuals. [258] The more recently identified antigens, as detected by auto-antibodies such as SPAG16 and KIR4.1, are only found in a fraction of the MS patients. [31,32] The relatively high prevalence of antiKIR4.1 positive patients, however, could not be reproduced in an independent study. [259] Our results show that $\mathrm{T}$ cell derived GM-CSF is mainly attributable to the non-Treg population. This is in concordance with a study using mice Tregs. [260] Moreover, our data show that Tregs can efficiently inhibit GM-CSF secretion by non-Treg CD4 ${ }^{+} \mathrm{T}$ cells from RRMS patients. Although, all patients were on IFN- $\beta$ therapy and therefore, a possible effect of this therapy on Treg function cannot be excluded, our data are in line with the results observed by Hartmann et al. revealing that Tregs can reduce $\mathrm{GM}-\mathrm{CSF}^{+} \mathrm{CD}^{+} \mathrm{T}$ cell frequencies. [254] 
Since culture supernatants were obtained from a previous study and $\mathrm{HC}$ were not included in the respective study design [116], comparison with HC was not possible. However, the Tresp/Treg cocultures of MS patients used in our study already strongly suppressed the GMCSF protein levels and therefore might have prevented to find a potential defect in suppression. Possibly, different Tresp/Treg ratios may unravel such an effect in future studies. Romio et al. showed that a possible mechanism of action of Tregs on GM-CSF secretion by Tresp might be exerted through the adenosine axis. [260] Adenosine can be converted from ATP by CD39 together with CD73, which are expressed on a Treg subset. [120, 121, 261, 262] This Treg subset seems to be functionally defective in MS patients $[121,122]$ and might, therefore, also be impaired in regulating $\mathrm{GM}-\mathrm{CSF}^{+} \mathrm{CD}^{+} \mathrm{T}$ cells.

An important immune modulator that seems to be associated with MS disease susceptibility and severity is vitamin D. $[70,147]$ Vitamin D might be critically involved in local regulation of $\mathrm{T}$ cell responses within the CNS, since calcitriol, the biological active vitamin D metabolite, can be generated locally by activated immune cells. [84] Additionally, infiltrating and perivascular T cells in MS lesions stained positive for the vitamin D receptor. [82] Our results show that calcitriol is able to inhibit GM-CSF expression and secretion by $\mathrm{CD}^{+} \mathrm{T}$ cells. This is in line with another study showing calcitriol dependent reduction of GM-CSF mRNA levels in a $\mathrm{T}$ cell line. [263] Although calcitriol equally reduces the fraction of GM-CSF positive $\mathrm{CD}^{+} \mathrm{T}$ cells in RRMS patients and HC, the reduction in GM-CSF protein levels is less effective in the RRMS patients as compared to HC. Since calcitriol has been known to inhibit T cell proliferation, the effects of calcitriol on GM-CSF might be linked to suppression of the activation or proliferation of the T helper cells (reviewed in [147]). However, a non-classical vitamin D receptor (VDR) response element has been observed in the promoter region of GM-CSF in a T cell line [263], suggesting a direct effect of calcitriol on GM-CSF transcription. It might be of interest to investigate possible (epi)genetic changes in this region in MS patients.

In conclusion, MS patients included in our study do not have an augmented GM-CSF ${ }^{+} \mathrm{CD}^{+}$ $T$ cell fraction in the circulation, but these cells can be detected in the CSF. Both Tregs and vitamin $\mathrm{D}$ seem to be able to down-regulate GM-CSF producing $\mathrm{CD}^{+} \mathrm{T}$ cells. The regulation of GM-CSF protein expression by calcitriol seems to be less efficient in RRMS patients as compared to controls. 


\section{Chapter}

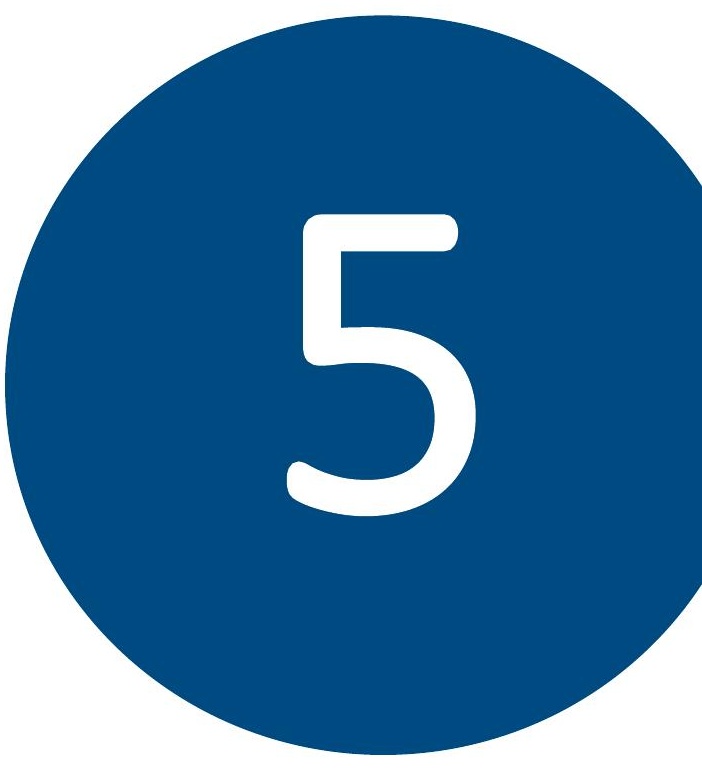

\section{Optimisation of the detection of IL-10 producing $\mathrm{T}$ cells by flow cytometry}

Published as: Intracellular IL-10 detection in T cells by flow cytometry: the use of protein transport inhibitors revisited;

Muris, A.H., Damoiseaux, J., Smolders, J., Cohen Tervaert, J.W., Hupperts, R., Thewissen, M.; Journal of Immunological Methods, J Immunol Methods. 2012 Jul 31;381(1-2):59-65. 


\section{Abstract}

In the past two decades, interleukin-10 (IL-10) has gained much attention as an important regulatory cytokine involved in self-tolerance. Functional assessment of IL-10 producing immune cells is traditionally done by stimulation and measurement of cytokine production by flow cytometry. Thereby a protein transport inhibitor like monensin is used to accumulate the cytokine of interest intracellularly. In this study we elaborated on the monensin effect on cytokine detection and focused on IL-10 detection in human $T$ cells.

Peripheral blood mononuclear cells (PBMC) of 32 study subjects were isolated and stimulated with PMA/ionomycin, in the absence and presence of monensin, and stained intracellularly for IFN-Y, IL-4, IL-10 and IL-17A.

Our results re-established that detection of IFN- $\mathrm{Y}^{+}$and $\mathrm{IL}-4^{+} \mathrm{T}$ cells benefited from the presence of monensin during stimulation. However, stimulation in the presence of monensin yielded lower proportions of $\mathrm{IL}^{-10^{+}} \mathrm{T}$ cells $(0.45 \%(0.28-0.80)$ versus $0.80 \%$ $(0.50-1.50)$ of $\mathrm{CD}^{+} \mathrm{T}$ cells, $\left.\mathrm{p}<0.01\right)$, although monensin addition did result in an increased MFI (2431 (1273-4959) vs. 1928 (1147-3760), $p<0.01)$. Detectable fractions of

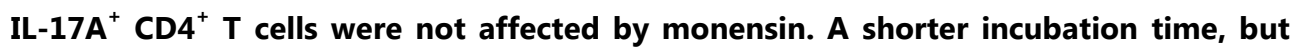
not lower monensin concentrations, was effective in improving the detection of IL-10 ${ }^{+}$ $T$ cells. We found a strong correlation between the fraction of $\mathrm{IL}^{-10^{+}} \mathrm{CD} 4^{+} \mathrm{T}$ cells in the presence and absence of monensin $(R=0.80, p<0.01)$. Next to this, also the detection of IL-10 ${ }^{+}$NK-T cells and IL- $10^{+}$monocytes, but not $\mathrm{IL}-10^{+} \mathrm{B}$ cells, is impaired in the presence of monensin.

This study shows that the effect of monensin on cytokine accumulation is time and cytokine dependent. Due to the use of monensin, previous research may have underestimated the number of IL-10 ${ }^{+}$leukocytes or may even have not been able to detect them at all. It is important to consider this for future research or when interpreting historical IL-10 data. 


\section{Introduction}

Interleukin-10 (IL-10) was first discovered in 1989 as a cytokine synthesis inhibitory factor (CSIF) produced by T helper 2 (Th2) cells. [264, 265] Nowadays, a variety of IL-10 producing leukocytes are identified, including subsets of $T$ cells, B cells, mast cells and phagocytic cells like eosinophils, monocytes/macrophages, and dendritic cells. IL-10 regulates the immune response by inhibiting the pro-inflammatory function of various innate and adaptive immune cells and of endothelial cells. [266, 267] In particular, the discovery of regulatory T cells (Treg) has further boosted the scientific interest in IL-10. Tregs are IL-10 producers, and there is some evidence that Tregs fail to develop or function in the absence of IL-10. [268] Furthermore, IL-10 can induce differentiation and trigger the suppressive function of the regulatory Tr1 subset. [266, 269-271] More recently, also IL-10 producing regulatory B cells and IL-10 producing regulatory natural killer (NK) cells have been discovered. [272-274] On the other hand, IL-10 has been described as a growth factor for activated B cells and therefore an increased production of this cytokine can play an important role in the onset of clinical manifestations of antibody-mediated auto-immune diseases such as systemic lupus erythematosus and myasthenia gravis. [275, 276] All together, this brought IL-10 to the attention as an important regulator in the process of inflammation and as a crucial player in the control of self-tolerance and auto-immunity. [266, 270]

Cytokine production can be assessed by multiple approaches. [277, 278] Since IL-10 can be produced by many different cell types it is desirable to appropriately identify the cellular source. Detection of IL-10 production at the single cell level by flow cytometry is a relatively rapid, highly sensitive and specific method to evaluate a large population of cells. [279, 280] Traditionally a protein transport inhibitor, such as monensin or brefeldin A (BFA), is used to prevent excretion, resulting in the accumulation of the cytokine of interest intracellularly. Monensin leads to anatomical disorganisation and non-specific disturbance of Golgi function and mainly affects the distal part of the Golgi complex, while BFA affects the pre-Golgi apparatus secretory pathway. [281-284] The use of a protein transport inhibitor in combination with intracellular staining makes it possible to identify subtypes of cytokine producing cells. Importantly, by using this method it is also possible to detect weakly expressed cytokines. [279] However, efficacy of cytokine detection can be time, activator and cytokine dependent, and is also dependent on the chosen protein transport inhibitor. In general monensin is more toxic than BFA. [285] However, with respect to the detection of IL-10, monensin has been advocated to be more effective than BFA in inducing IL-10 accumulation in T cells.

The use of protein transport inhibitors and especially monensin was traditionally tested and optimized for cytokines like IL-2, IL-4 and interferon-gamma (IFN- - ). In these experiments monensin enhanced fluorescence intensity, increased the number of detectable cells and delayed the peak of cytokine production. $[279,285,286]$ We noticed that monensin may actually hamper IL-10 detection. Therefore, we studied in detail the effects of monensin on 
cytokine detection, in particular detection of IL-10 in T-cells, and investigated whether we could improve detection of human IL-10 producing immune cells by lowering monensin concentrations or shortening monensin incubation time.

\section{Material and methods}

\section{Subjects}

We included 32 study subjects. Nineteen of them were female (59.4\%), 13 were male (40.6\%) and the median age of the whole population was 34.5 years (range from 22-73 years). Informed consent was acquired from all subjects.

\section{Isolation of PBMCs}

Peripheral blood samples from study subjects were collected in a $10 \mathrm{~mL}$ sodium heparin blood sampling tube (BD Bioscience, Breda, the Netherlands). Peripheral blood mononuclear cells (PBMC) were isolated using Ficoll-density gradient (Histopaque; Sigma Aldrich, Zwijndrecht, the Netherlands) and centrifugation, performed as described before. [191] The isolated PBMCs were kept in RPMI glutamax medium (Gibco Invitrogen, Breda, the Netherlands) supplemented with $10 \%$ foetal calf serum (Greiner Bio-One, Alphen a/d Rijn, the Netherlands), $1 \%$ non-essential amino acids (Gibco Invitrogen), 1\% sodium pyruvate (Gibco Invitrogen) and $2 \%$ penicillin-streptomycin (Gibco Invitrogen).

\section{Stimulation of PBMC with PMA/ionomycin in the absence or presence of monensin}

PBMCs were stimulated for 5 hours with PMA (50 ng/mL, Sigma Aldrich) and ionomycin (1 $\mu \mathrm{g} / \mathrm{mL}$, Sigma Aldrich) in the absence or presence of monensin (BD Biosciences) or BFA (Sigma Aldrich) and placed in a $37^{\circ} \mathrm{C}$ incubator with a humidified atmosphere containing $95 \%$ air and $5 \% \mathrm{CO}_{2}$. Monensin was used in the recommended concentration of $1.25 \mu \mathrm{g} / \mathrm{mL}$ or was further diluted to concentrations ranging from $0 \mu \mathrm{g} / \mathrm{mL}$ to $1.25 \mu \mathrm{g} / \mathrm{mL}$ as indicated in the figures. BFA was used in a concentration of $5 \mu \mathrm{g} / \mathrm{mL}$.

\section{Intracellular staining}

PBMCs were stained with anti-CD3-horizon450 (BD Biosciences), anti-CD8-APC-H7 (BD Biosciences) and anti-CD19-FITC (BD Biosciences) to distinguish $\mathrm{CD}^{+} \mathrm{CD} 8^{-}$lymphocytes (CD4 ${ }^{+} \mathrm{T}$ cells), $\mathrm{CD}^{+} \mathrm{CD}^{+}$lymphocytes (CD8 ${ }^{+} \mathrm{T}$ cells) and $\mathrm{CD} 19^{+} \mathrm{CD} 3^{-}$lymphocytes (B-cells). Monocytes were identified based on the FSC/SSC scatter and absence of CD3 staining. $\mathrm{CD}^{+}{ }^{+} \mathrm{CD} 56^{+}$lymphocytes (natural killer-like T cells (NK-T cells)) were stained using anti-CD3horizon 450 and anti-CD56-PE (BD Biosciences). PBMCs were fixed and permeabilised (Cytofix/Cytoperm, BD Biosciences) and stained intracellularly with anti-IL-4-PE (Biolegend, Uithoorn, the Netherlands), anti-IFN- $\nu$-FITC (BD Biosciences), anti-IFN- $\nu$-PE (BD Biosciences), anti-IL-17A-PerCP-Cy5.5 (Biolegend) and anti-IL-10-APC (Biolegend). The activation status of cells was assessed by anti-CD69-PE-Cy7 (Biolegend). Samples were analysed on a FACS Canto II flow cytometer (BD Biosciences). 


\section{Statistical analysis}

GraphPad Prism 5 (GraphPad Software Inc., La Jolla CA, USA) was used to construct figures and perform statistics. Of continuous variables, the median value and corresponding range (min-max) are provided. Differences between two related samples were assessed using the Wilcoxon signed-rank test. Correlations were assessed with the Spearman correlation coefficient. A p-value $<0.05$ was considered statistically significant.

\section{Results}

\section{Monensin impairs the detectable amount of $\mathrm{IL}_{-10}{ }^{+} \mathrm{CD}^{+}$and $\mathrm{IL}_{-10^{+}} \mathrm{CD}^{+} \mathrm{T}$ cells}

As is evident from Figure $1 \mathrm{~A}$, monensin positively affects both the relative amount as well as the median fluorescence intensity (MFI) of IFN $-\nu$ producing cells, whereas the relative amount of IL-10 producing cells is reduced. Indeed, BFA is comparable to monensin in the detection of IFN $-\nu^{+} \mathrm{CD} 4^{+} \mathrm{T}$ cells, but negatively influences the detectable relative amount of $\mathrm{IL}-10^{+} \mathrm{CD} 4^{+} \mathrm{T}$ cells. Following these observations we studied the effect of monensin addition on the detection of IFN- $\gamma$, IL-4 (the prototype cytokines for Th1 and Th2 cells, respectively), IL-10 and IL-17A producing cells by intracellular flow cytometry. The fraction of cytokine producing cells and MFI was highly variable between donors (Table 1). To evaluate the effect of monensin, we divided the fraction (or MFI) of cytokine positive cells measured in the absence of monensin by the fraction (or MFI) in the presence of monensin, for each cytokine and each donor. Calculated monensin ${ }^{-} /$monen $\sin ^{+}$ratios were below 1 for both IFN $-\gamma$ and IL4: 0.53 and 0.79 (both $p<0.01$ ), respectively (Figure $1 B$ ), implying that both IFN- $\gamma$ and IL-4 producing $\mathrm{T}$ cells are better detectable in the presence of monensin. Also the ratio of Th1/Th2 cells, defined as the IFN $-\gamma^{+} / \mathrm{IL}-4^{+} T$ cell ratio, was significantly higher $(p<0.01)$ when measured in the presence of monensin (median=3.75), as compared to the ratio in the absence of monensin (median=2.78). This implies that IFN- $\gamma$ detection benefits more from monensin addition than IL-4 detection. Interestingly, the median monensin $/$ monensin $^{+}$ratio of the fraction of IL10 ${ }^{+} \mathrm{CD} 4^{+}$T-cells was $1.75(p<0.01)$ (Figure 1B). Detection of IL-17A ${ }^{+} \mathrm{CD} 4^{+}$ $T$ cells was not affected by the addition of monensin, the median monensin ${ }^{-} /$monensin $^{+}$ratio was 1.00 . Furthermore, the monensin ${ }^{-} /$monen $\sin ^{+}$ratio's for IFN- - , IL-4, IL-10 and IL-17A were significantly different $(p<0.01)$ from each other, indicating that the consequences of monensin addition on the detectable fraction of cytokine producing $\mathrm{CD} 4^{+} \mathrm{T}$ cells are highly cytokine dependent. The difference in vulnerability to monensin of different T-cell subsets could not be attributed to differential activation status, since all T-cell subsets expressed similar levels of CD69, after activation (data not shown). Furthermore, these results were independent of the monensin batch used, or the general status of the sample (data not shown). Similar findings were obtained in the $\mathrm{CD} 8^{+} \mathrm{T}$ cell population (data not shown). 

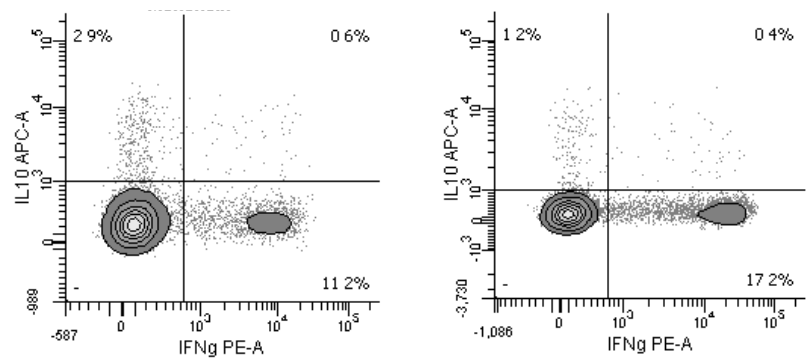

B

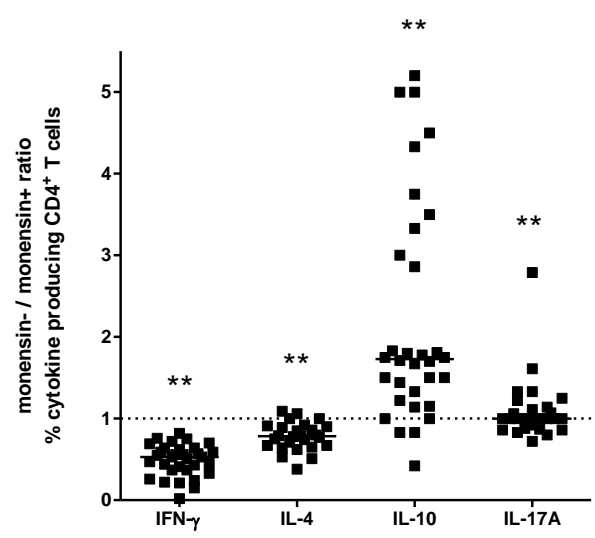

C
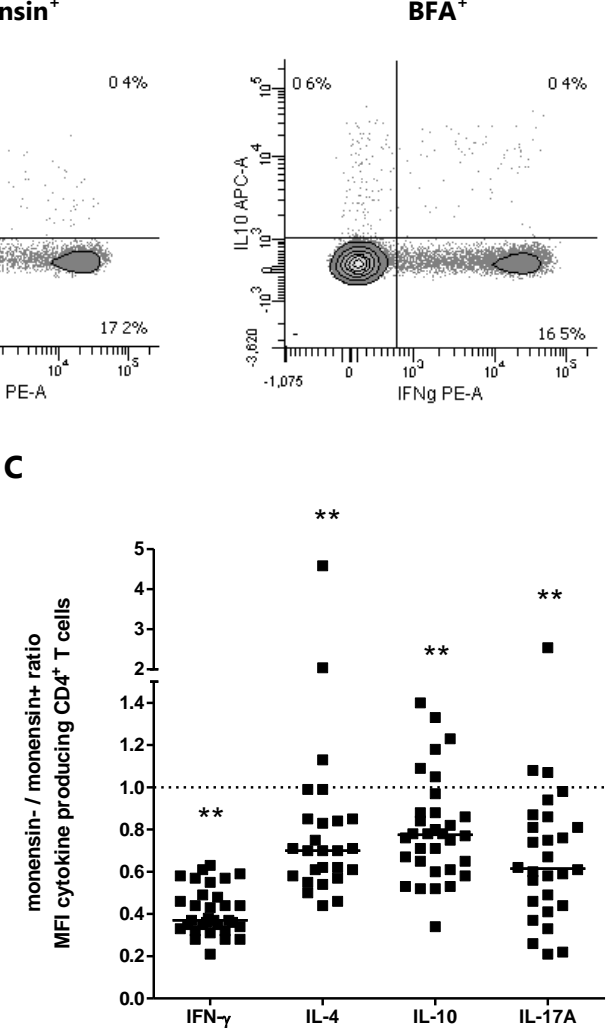

Figure 1. Effects of monensin or BFA on cytokine production and MFI in human $\mathrm{CD} 4^{+} \mathrm{T}$ cells.

A) Representative dot plots of IL-10 and IFN-y production by human $\mathrm{CD}^{+} \mathrm{T}$ cells in absence and presence of monensin or BFA B) monensin $/$ monensin $^{+}$ratio of fraction of cytokine producing $C D 4^{+} T$ cells $\left.(n=32) C\right)$ monensin $/$ monensin ${ }^{+}$ratio of MFI of cytokine producing $C D 4^{+} T$ cells $(n=32)$

${ }^{*} p<0.05,{ }^{* *} p<0.01 ;$ monensin ${ }^{*}$ compared to monensin ${ }^{+}$condition; CD; cluster of differentiation; IFN; interferon; IL; interleukin; MFI: median fluorescence intensity; monensin/monensin ${ }^{+}$ratio: fraction (or MFI) of cytokine positive cells measured in the absence of monensin divided by the fraction (or MFI) in the presence of monensin

In addition, we could confirm that monensin increases the MFI of IFN- $\gamma$ and IL-4 producing $\mathrm{CD}^{+} \mathrm{T}$ cells: this is expressed in a monensin ${ }^{-} /$monensin $^{+}$ratio of 0.37 and 0.70 (both $\mathrm{p}<0.01$ ) for IFN $-\gamma^{+} \mathrm{CD} 4^{+} \mathrm{T}$ cells and IL- $4^{+} \mathrm{CD} 4^{+} \mathrm{T}$ cells, respectively (Figure $1 \mathrm{C}$ ). Also the MFI of IL-10 ${ }^{+}$

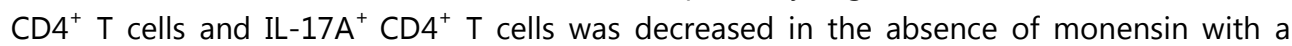
monensin ${ }^{-} /$monensin ${ }^{+}$ratio of 0.77 and 0.62 (both $p<0.01$ ), respectively (Figure $1 C$ ). 
Table 1. Fraction and MFI of cytokine producing $\mathrm{CD}^{+} \mathrm{T}$ cells in the presence (monensin ${ }^{+}$) and absence of monensin (monensin $)(n=32)$.

\begin{tabular}{lllllll} 
& Fraction & & & MFI & & \\
Cytokine & Monensin & & & & \\
Monensin & ${ }^{+}$ & & Monensin & & Monensin $^{+}$ & \\
IFN-y & $5.05 \%$ & $8.90 \%$ & $* *$ & 1441 & 4054 & $*$ \\
& $(1.00-40.10)$ & $(4.10-78.18)$ & & $(1008-4395)$ & $(1867-10102)$ & \\
IL-4 & $2.32 \%$ & $2.57 \%$ & $* *$ & 1766 & 2587 & $*$ \\
& $(0.73-7.80)$ & $(1.10-9.90)$ & & $(1010-5108)$ & $(1058-5376)$ & \\
IL-10 & $0.80 \%$ & $0.45 \%$ & $* *$ & 1928 & 2431 & $*$ \\
& $(0.08-3.80)$ & $(0.05-2.10)$ & & $(1147-3760)$ & $(1273-4959)$ & \\
IL-17A & $0.50 \%$ & $0.50 \%$ & & 6537 & 10359 & $*$ \\
& $(0.13-2.90)$ & $(0.15-2.70)$ & & $(417-21197)$ & $(1777-36010)$ & \\
\hline
\end{tabular}

${ }^{* *} p<0.01$; monensin ${ }^{-}$compared to monensin ${ }^{+}$condition; IFN: interferon; IL: interleukin; MFI: median fluorescence intensity

\section{Monensin inhibits detection of IL-10 producing $\mathbf{T}$ cells at low concentrations}

Next, we investigated whether we could enhance the sensitivity of detection of IL-10 ${ }^{+} \mathrm{CD} 4^{+} \mathrm{T}$ cells, without losing sensitivity for IFN $-\gamma^{+} \mathrm{CD}^{+} \mathrm{T}$ cell detection, by lowering the monensin concentration. For evaluation we normalised all fractions to the detected fraction of cytokine producing cells in the recommended monensin concentration of $1.25 \mu \mathrm{g} / \mathrm{mL}$. This was done for each cytokine and for each donor separately.

For IFN- $\gamma$, we observed a monensin concentration dependent loss of sensitivity (Figure $2 \mathrm{~A}$ ): the higher the monensin concentration, the higher the fraction of detectable IFN $-\gamma^{+} \mathrm{CD} 4^{+} \mathrm{T}$ cells, although these differences were not statistically different. The detectable fraction of IL10 producing $\mathrm{CD}^{+} \mathrm{T}$ cells was comparable in the different monensin concentrations tested (Figure 2B). These data show that lowering the monensin concentration did not enhance the sensitivity of the detection of IL-10 producing $C D 4^{+}$T cells without affecting the detection of IFN- $p$ producing $\mathrm{CD} 4^{+} \mathrm{T}$ cells.

\section{Monensin is most effective in detecting IL-10 producing CD4 ${ }^{+} \mathrm{T}$ cells after 1 or 2 hours of incubation}

Another option to circumvent the negative effects of monensin could be to use a shorter monensin incubation time. PBMCs were stimulated for 5 hours with PMA/ionomycin, and monensin $(1.25 \mu \mathrm{g} / \mathrm{mL})$ was present during stimulation for 0 to 5 hours. For evaluation we normalised all obtained fractions (or MFI) to the fraction (or MFI) of cytokine producing $\mathrm{CD}^{+} \mathrm{T}$ cells in the 5 hours monensin incubation protocol. This was done for each cytokine and for each donor separately (Figure 3).

Detection of IFN $-\gamma^{+} \mathrm{CD}^{+} \mathrm{T}$ cells was optimal when monensin was added for 3 to 5 hours. With shorter incubation times, the fraction of IFN $-\nu^{+} \mathrm{CD} 4^{+} \mathrm{T}$ cells decreased to a median ratio of 0.75 at 1 hour of incubation $(p<0.05)$ (Figure $3 \mathrm{~A}$ ). The detection of IL- $4^{+} \mathrm{CD} 4^{+} \mathrm{T}$ cells 
A

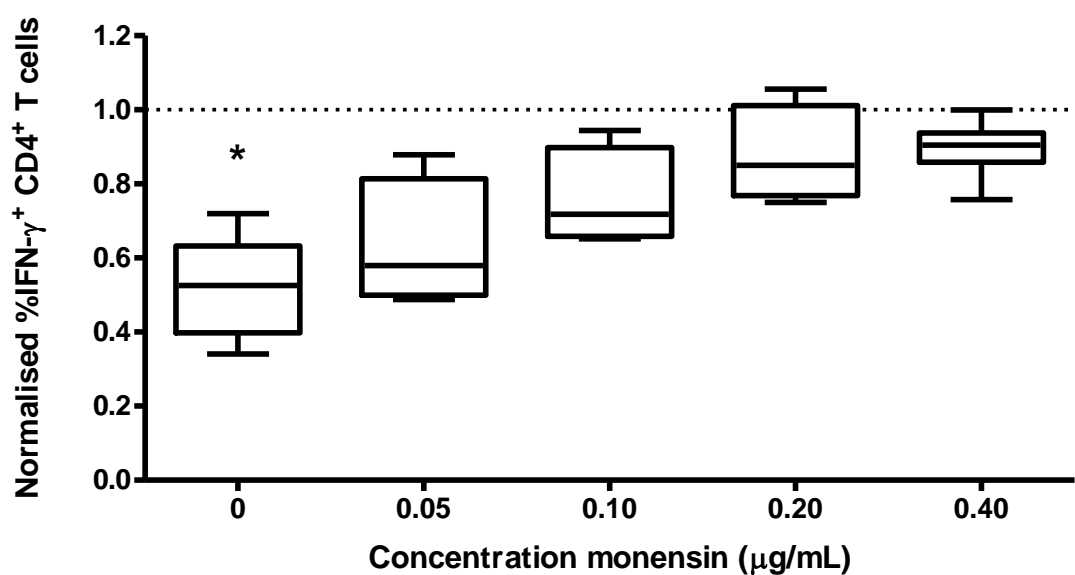

B

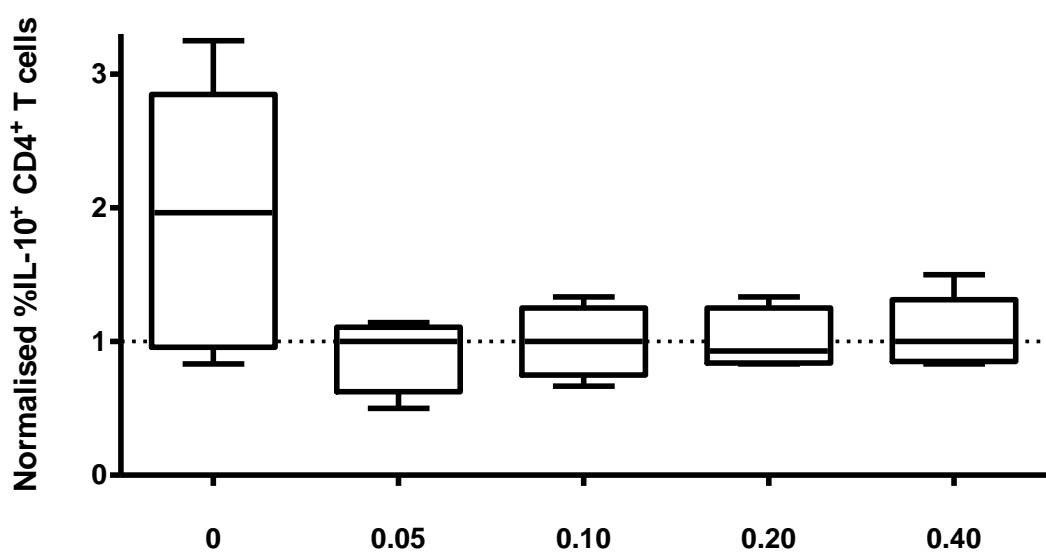

Concentration monensin $(\mu \mathrm{g} / \mathrm{mL})$

Figure 2. Effect of monensin concentration on detection of cytokine producing $\mathrm{CD} 4^{+} \mathrm{T}$ cells.

Normalised fractions of A) IFN-y producing $\mathrm{CD}^{+}{ }^{+} \mathrm{T}$ cells B) IL-10 producing $\mathrm{CD}^{+}{ }^{+} \mathrm{T}$ cells; in the various monensin concentrations tested $(n=6)$. The recommended monensin concentration $(1.25 \mathrm{mg} / \mathrm{mL})$ was used to normalise detected fractions measured in the various monensin concentrations tested.

${ }^{*} p<0.05$; detected fraction in tested monensin incubation time compared to detected fraction with 5 hours of monensin incubation; IFN: interferon; IL: interleukin 
showed a trend towards an optimum at a monensin incubation period of 2 to 5 hours (Figure $3 \mathrm{~B}$ ). In general, the relative amount of $\mathrm{IL}-10$ producing $\mathrm{CD}^{+} \mathrm{T}$ cells increased with a

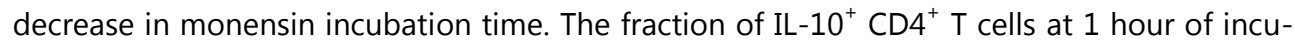
bation versus 5 hours of incubation was $2.91(p<0.05)$ (Figure 3C). The percentage of IL-17A producing cells was not influenced by the monensin incubation time with a normalised fraction of IL-17A ${ }^{+} \mathrm{CD} 4^{+} \mathrm{T}$ cells of about 1 for all conditions (Figure 3D). We have shown before (Figure $1 \mathrm{C}$ ) that although the fraction of $\mathrm{IL}-10^{+} \mathrm{CD} 4^{+} \mathrm{T}$ cells increased in the absence of monensin, this was at the expense of the MFI. Therefore, we assessed whether this reduction in MFI was influenced by the monensin incubation time. The MFI of IFN $-\gamma^{+} \mathrm{CD}^{+} \mathrm{T}$ cells was comparable when monensin was added for 3 to 5 hours. A monensin incubation time of 2 hours or shorter affected the MFI of IFN $-\gamma^{+} \mathrm{CD}^{+} \mathrm{T}$ cells significantly (Figure 3A). This was also the case for the MFI of IL- $4^{+} \mathrm{CD} 4^{+} \mathrm{T}$ cells which decreased time dependently when monensin was present for 3 hours or less $(p<0.05)$ (Figure $3 B$ ). For IL-10 producing CD4 ${ }^{+} T$ cells the MFI also decreased with shorter incubation times $(p<0.05$ for 1 and 2 hours incubation time) (Figure $3 \mathrm{C}$ ). The MFI of IL-17A producing $\mathrm{CD}^{+} \mathrm{T}$ cells was not affected by the addition of monensin (Figure 3D).

The fraction of $\mathrm{IL}-1 \mathrm{O}^{+} \mathrm{CD} 4^{+} \mathrm{T}$ cells detected in the absence of monensin is strongly correlated with the fraction of $\mathrm{IL}-10^{+} \mathrm{CD} 4^{+} \mathrm{T}$ cells in the presence of monensin

Since most studies on intracellular IL-10 detection in $\mathrm{CD}^{+} \mathrm{T}$ cells were done in the presence of monensin, we investigated the relationship between the IL-10 CD4 ${ }^{+} \mathrm{T}$ cell fraction measured in the absence and presence of monensin. As is shown in Figure 4, the fraction of IL-10 $\mathrm{CD}^{+} \mathrm{T}$ cells detected in the absence of monensin was strongly correlated with the fraction of IL- $10^{+} \mathrm{CD}^{+} \mathrm{T}$ cells detected in the presence of monensin (Spearman R 0.80, $\mathrm{p}<0.01$ ). Also the fractions of other cytokine producing $\mathrm{CD}^{+}{ }^{+} \mathrm{T}$ cells detected in the absence of monensin were correlated with the fraction detected in the presence of monensin: Spearman $\mathrm{R}$ of 0.78 $(p<0.01), 0.91(p<0.01)$ and $0.94(p<0.01)$ for IFN- $p, I L-4$ and IL-17A respectively. Also the Th1/Th2 ratio's in the absence and presence of monensin were significantly correlated $(R$ $0.80, p<0.01)$.

\section{Detection of IL-10 ${ }^{+}$monocytes and IL-10 ${ }^{+}$NK-T cells, but not IL-10 ${ }^{+}$B cells, seem to be affected by monensin}

To investigate whether the monensin effect on IL-10 production was T cell specific, we additionally studied other IL-10 producing leukocytes directly ex vivo. To evaluate the effect of monensin addition, we divided the fraction (or MFI) of IL-10 positive cells measured in the absence of monensin by the fraction (or MFI) in the presence of monensin, for each donor. Besides the negative effects on IL-10 detection in T cells $(p<0.01)$, monensin has detrimental effects on the detection of IL- $10^{+} \mathrm{NK}-\mathrm{T}$ cells, and IL- $10^{+}$monocytes (median monensin /monensin ${ }^{+}$ratios of $1.69(p<0.01)$ and $1.38(p<0.01)$, respectively). B cells, however, seemed to benefit from the addition of a protein transport inhibitor: the median monensin /monensin ${ }^{+}$ratio was $0.60(p<0.01)$. (Figure 5) 

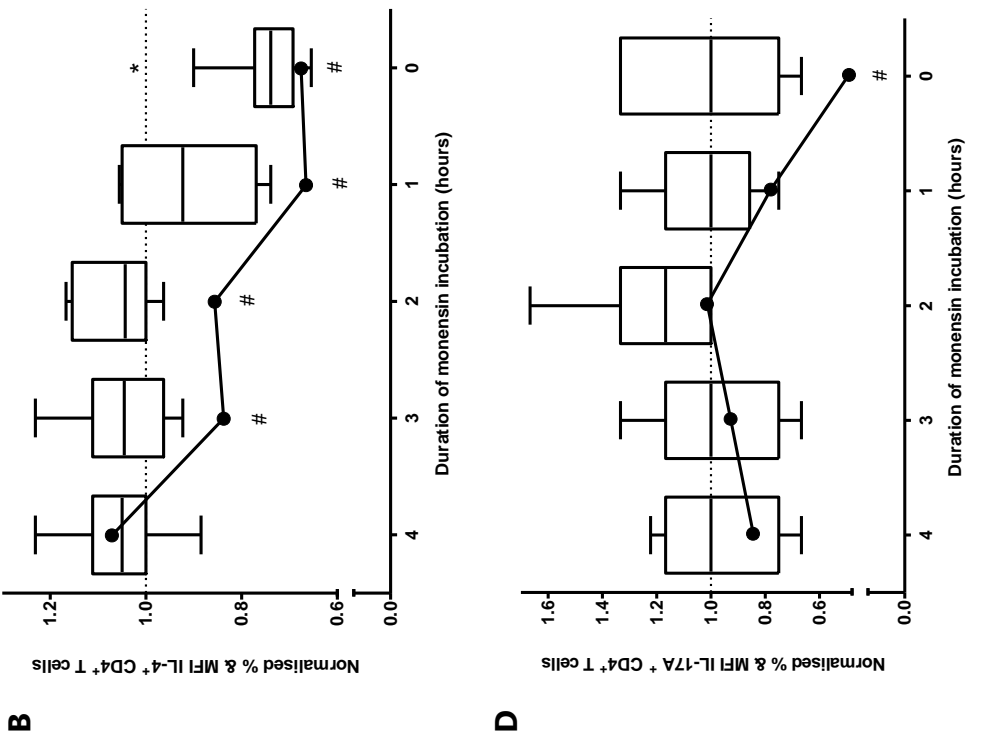

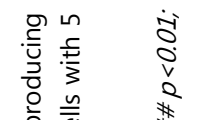

흔

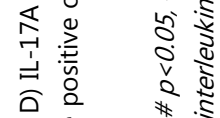

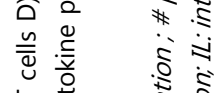

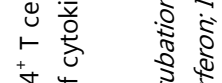

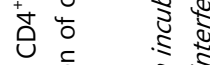

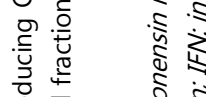

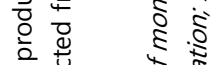

가

ㄴㅇ के

ป

券

$\circ$
$\square$

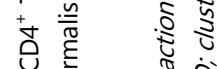

वे के

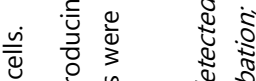

$\infty$

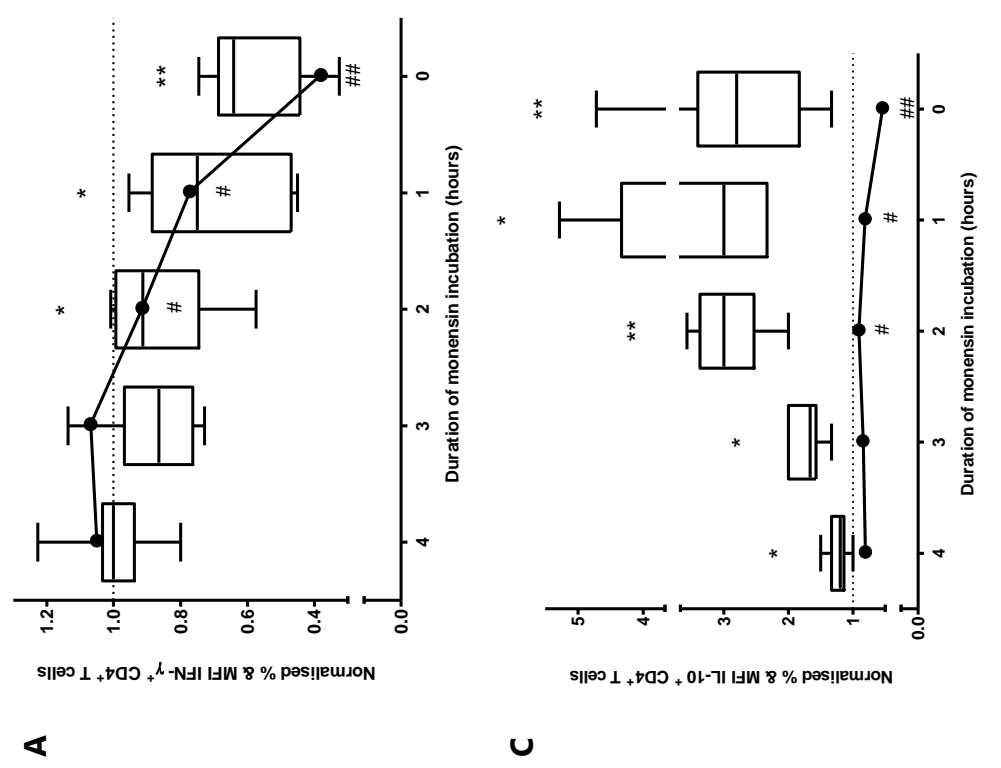

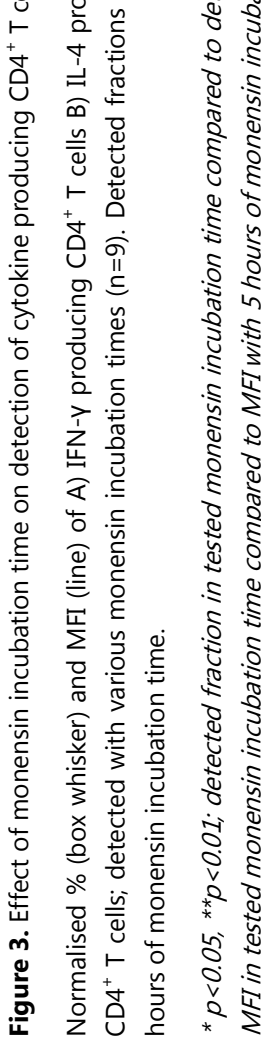




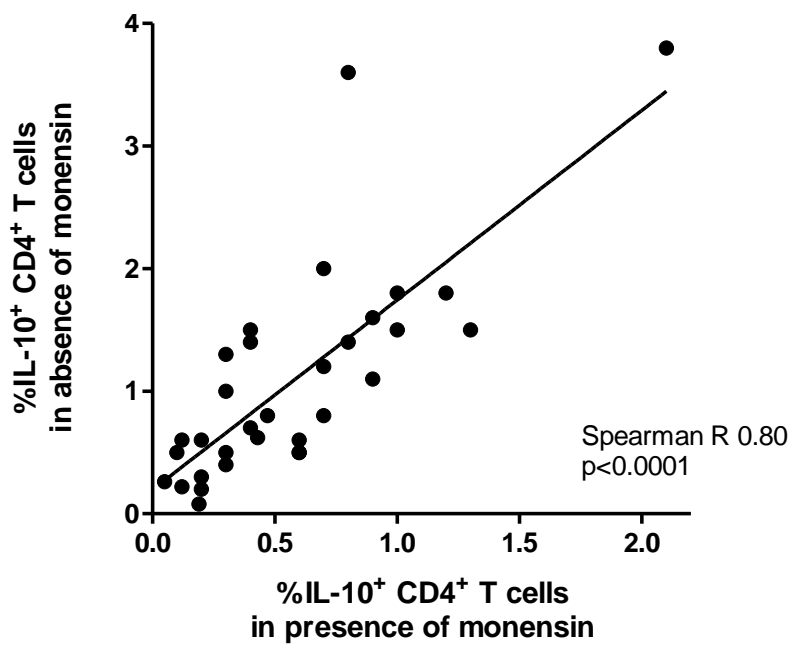

Figure 4. Correlation between fraction of $\mathrm{IL}-10^{+} \mathrm{CD} 4^{+} \mathrm{T}$ cells in the presence and absence of monensin.

Fractions of $\mathrm{IL}-10^{+} \mathrm{CD} 4^{+} \mathrm{T}$ cells in the absence of monensin are plotted against the fraction of IL-10 ${ }^{+} \mathrm{CD} 4^{+} \mathrm{T}$ cells in the presence of monensin $(n=32)$. Monensin was used in the recommended concentration $(1.25 \mu \mathrm{g} / \mathrm{mL}) \mathrm{and}$ the standard incubation time of 5 hours.

CD: cluster of differentiation; IL: interleukin

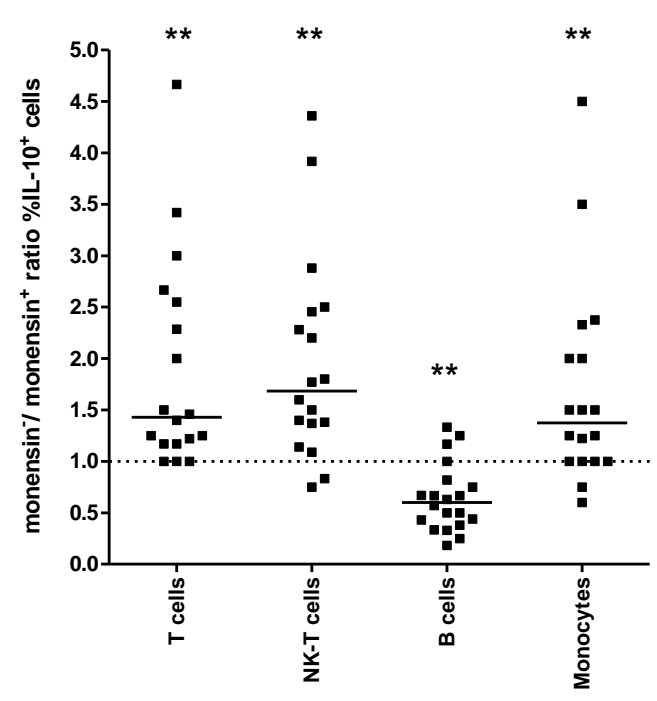

Figure 5. Effect of monensin on IL-10 producing leukocytes.

Monensin ${ }^{-} /$monensin ${ }^{+}$ratios of fraction of IL-10 producing cells in presence or absence of monensin $(n=18)$. Directly ex vivo, T cells are defined as $\mathrm{CD}^{+}$lymphocytes, NK-T cells as $\mathrm{CD} 3^{+} \mathrm{CD} 56^{+}$lymphocytes, B cells as CD19 ${ }^{+}$lymphocytes and monocytes were identified based on the FSC/SSC scatter and absence of CD3 staining

${ }^{*} p<0.05{ }^{* *} p<0.01 ;$ monensin ${ }^{-}$compared to monensin ${ }^{+}$condition; IL: interleukin; NK: natural killer 


\section{Discussion and Conclusion}

In this study, we show that the effect of monensin on cytokine accumulation is time and cytokine dependent. In contrast to the known improvement of cytokine detection for IFN- $\gamma$ and IL-4 $[279,285,286]$, monensin impairs the detectable fraction of IL- $10^{+} \mathrm{T}$ cells, whereas detection of IL-17A producing T cells is not affected. Monensin, though, increases the IL-10 per cell (expressed by an increased MFI), as is the case for other cytokines. Apparently, monensin has a highly cytokine dependent effect on the different subsets of cytokine producing cells, and this is confirmed by significant differences between the monensin /monensin ${ }^{+}$ratios of the detectable fraction of IFN- $\gamma$, IL-4, IL-10 and IL-17A producing CD4 ${ }^{+}$ T cells.

Monensin impaired detection of IL- $10^{+} \mathrm{CD}^{+} \mathrm{T}$ cells in 27 of the 32 studied subjects. However, we observed a high donor-to-donor variation in the effect monensin had on the detection of IL-10. The extent of the monensin effect on cytokine detection did not correlate between the different cytokines within the same sample. Moreover, the inter-individual variation was not caused by differences in the general status of the sample or the size of the $\mathrm{IL}-10^{+} \mathrm{CD} 4^{+} \mathrm{T}$ cell population.

To circumvent the drop in IL-10 detection without affecting the detection of other intracellular cytokines we tested either the use of lower monensin concentrations or a shorter monensin incubation time. A 25 -fold lower monensin concentration had detrimental effects on IFN- $\gamma$ detection but did not improve IL-10 detection in $\mathrm{CD}^{+} \mathrm{T}$ cells. Incubation with monensin for a shorter time period, e.g. 2 hours, resulted in improved detection of IL-10 producing $T$ cells $(p<0.05)$. This might be a good alternative for the simultaneous detection of IFN- $\gamma$, IL-4, IL-10 and IL-17A producing cells in one tube. A possible disadvantage of this approach is that distinguishability of cytokine positive cells, expressed in MFI, might be worse. Otherwise, when only the IL- $10^{+} \mathrm{T}$ cell is of interest, absence of monensin in the intracellular FACS protocol should be considered. An extra advantage then is that quantification of excreted cytokines into the supernatant by for example ELISA will be possible. Besides, there are also other ways to detect IL-10 producing cells like the recently described single cell secretion analysis, which is based on droplet based micro fluidic platforms. [287] Finally, although these lymphocyte subsets are usually stimulated by more cell specific stimuli and excretion of cytokines is prevented by using BFA instead of monensin, we showed that the effect of monensin is immune cell specific: monensin seems to impair detection of IL- $10^{+} \mathrm{T}$ cells, IL- $10^{+}$NK-T cells, and IL- $10^{+}$monocytes, but not of IL- $10^{+}$B cells.

The mechanism behind this impaired detection of IL-10 producing $T$ cells is at present unclear. Monensin is known to inhibit the secretion of cytokines, which are then accumulated intracellularly. This is in agreement with our data which show an increased MFI upon monensin usage for all cytokines tested. Monensin is known to be toxic, probably due to the block in Golgi function. [285, 286, 288, 289] Recently, monensin was shown to induce a Golgi stress response, which is manifested by remodelling of the expression of genes involved in lipid synthesis, extension of Golgi capacity and apoptosis. [288, 290] In addition, there is 
conflicting evidence for the (direct or indirect) effects of monensin on the endoplasmic reticulum (ER). [290, 291] The ER stress response and related unfolded protein response have been shown to induce an increase in pro-inflammatory cytokine production. [292] We propose that monensin triggers, directly via the Golgi stress response or indirectly via the ER stress response, a pro-inflammatory cytokine shift, which could explain the increase in the detection of pro-inflammatory cytokines and the decrease in the detection of $\mathrm{IL}-10^{+} \mathrm{T}$ cells in the presence of monensin. The incubation-time experiments may underline this hypothesis, since a 2 hours monensin incubation time may be too short to affect gene expression and translation. On the cellular level, different cell types may be variably vulnerable to the induction of stress responses by monensin, for example by expression of microRNAs, and the same stress responses may result in a different outcome. In IL-10 positive T cells, these stress responses may preferentially result in apoptosis. However, in PBMCs, the fraction of IL10 producing $T$ cells is too low to specifically test toxic effects of monensin in these cells. Alternatively, monensin was shown to delay cytokine production in the cell. [279] It can be speculated that IL-10 production is more delayed than IFN- $\gamma$ production. However, our data do not support this explanation for the impaired IL-10 detection in T cells.

Future research into IL-10 production by leukocytes should take into account that monensin reduces the proportion of $\mathrm{IL}-10^{+}$cells detected with intracellular flow cytometry, or even makes them undetectable. Due to the use of monensin, previous research may have underestimated the number of IL-10 $10^{+}$leukocytes. It is important to consider this when interpreting historical IL-10 data. 



\section{Chapter}

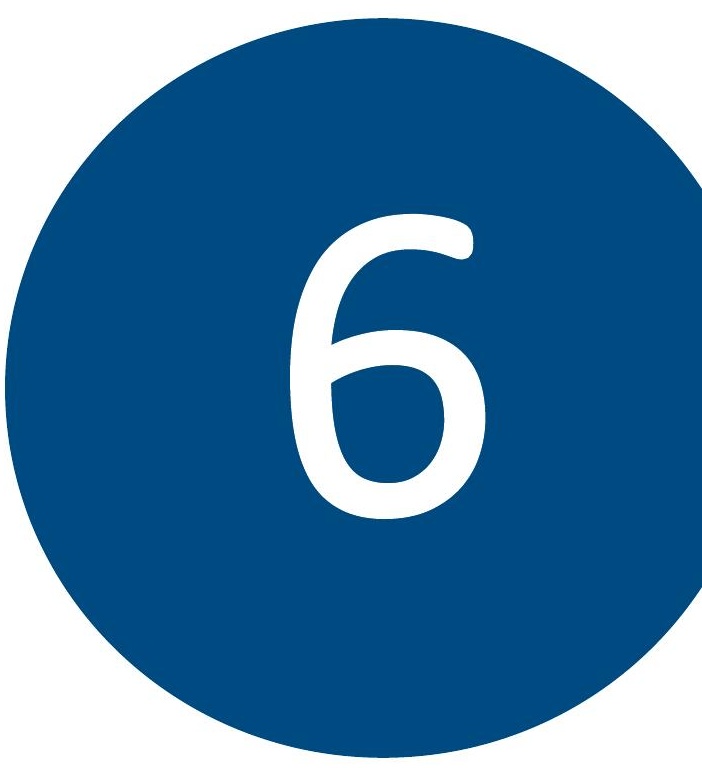

\section{Immune regulatory effects of high dose vitamin D supplementation in patients with relapsing remitting multiple sclerosis}

Submitted as: Immune regulatory effects of high dose vitamin D3 supplementation in a randomised controlled trial in relapsing remitting multiple sclerosis patients receiving IFN- $\beta$; the SOLARIUM study

Muris, A.H., Smolders J.; Rolf L., Thewissen M., Hupperts R., Damoiseaux, J. on behalf of the SOLARIUM study group. 


\section{Abstract}

Multiple sclerosis (MS) is characterized by a disturbed homeostasis of the circulating immune cell compartment and low serum levels of vitamin D are associated with an increased disease activity. Vitamin $D$ has been hypothesized to promote the maintenance of immune homeostasis. High dose vitamin $D_{3}$ supplementation could therefore be of benefit to patients with MS. The SOLAR study investigated the effects of high dose vitamin $D_{3}$ supplementation on clinical outcomes in a randomised controlled trial. Here we present the immune regulatory effects, investigated in the SOLARIUM sub-study.

Peripheral blood mononuclear cells (PBMC) were isolated from 53 Dutch relapsing remitting (RR)MS patients treated with IFN- $\beta-1 a$, who participated in the SOLAR study. Thirty of them received high dose vitamin $D_{3}$ supplementation and 23 received placebo during a follow-up period of 48 weeks. Lymphocytes were phenotypically characterized by flow cytometry and in vitro pro- and anti-inflammatory cytokine secretion in culture supernatant was assessed in the presence or absence of $1,25(\mathrm{OH})_{2} \mathrm{D}_{3}$ using Luminex technology. Changes in immune regulatory parameters were determined within subjects as well as between treatment groups.

First, the number of cells in the regulatory immune cell compartment (including $\mathbf{n T r e g}$, iTreg and Breg) was not altered upon high dose vitamin $D_{3}$ supplementation. Second, proportions of $T$ helper subsets were not affected by vitamin $D_{3}$, except for the proportion of IL- $4^{+}$Th cells, which decreased in the placebo but not in the vitamin $D_{3}$ group. $T$ cell cytokine secretion increased, most pronounced for IL-5 and latency activated protein of TGF- $\beta$, in the placebo group but not in the vitamin $D_{3}$ group. Lymphocytes remained equally reactive to in vitro $1,25(\mathrm{OH})_{2} \mathrm{D}_{3}$ addition after 48 weeks of follow-up.

In conclusion, high dose vitamin $D_{3}$ supplementation did not result in a relative increase in lymphocytes with a regulatory phenotype. However, this study supports the hypothesis that vitamin $D$ contributes to the maintenance of immune homeostasis by preventing a further disturbance of the $T$ cell compartment taking place during the early disease course in MS. Clinical studies will eventually indicate if the subtle differences between the treatment groups in this study, also result in improved clinical outcome. 


\section{Introduction}

Main challenges in multiple sclerosis (MS) research are to further unravel the underlying pathogenic mechanisms of this still poorly-understood disease and to make treatments more effective while minimising the associated serious side effects. Genetic [14], epidemiological $[71,74]$, clinical $[72,75,199,200]$ and immunological $[131,154]$ data have pointed towards a beneficial role of vitamin D in MS risk, and disease activity. Since most MS patients have low vitamin $D$ levels, vitamin $D$ supplementation may be an effective and safe (add-on) treatment option in MS. The SOLAR study assessed the efficacy of high dose vitamin $D_{3}$ as add-on therapy to interferon-beta-1a (IFN- $\beta$ - 1 a) on clinical outcome, i.e. disease activity free status, in 232 patients with early relapsing remitting MS (RRMS) in 11 countries in Europe (NCT01285401). [136] In this paper we present the results on the immune modulating effects of high dose vitamin $D_{3}$ supplementation investigated in the Dutch sub-study SOLARIUM (SOLAR ImmUne Modulating effects).

MS is a devastating inflammatory disease of the central nervous system (CNS). In its most prevalent phenotypic entity, called RRMS, the disease course is characterized by periods of neurological deterioration (relapses), followed by periods of recovery (remissions). In particular, this subtype is considered to be of auto-immune origin, but neither the target nor the exact aetiology has been unravelled to date. The immunopathological hypothesis currently most widely accepted is that auto-reactive lymphocytes become activated in the secondary lymphoid tissues and, after entering the CNS, become reactivated. Via a positive feedback loop, a pro-inflammatory environment arises, resulting in an attack by the immune system that damages myelin and neurons, eventually leading to loss of neurological function. [105, 109 , 293] For many years, MS was regarded to be a T helper 1 (Th1; mainly IFN- $\gamma$ producing Th cells) mediated disease. [36] More recently, Th17 cells, producing interleukin 17 (IL-17) $[36,37]$, and GM-CSF producing Th cells [38, 39] are held responsible for the encephalitogenic reaction. Outside the CNS, in the circulating immune system, MS is characterized by a disturbed lymphocyte homeostasis, with a diminished function of regulatory $T$ cells (Treg) $[117,294]$, a decrease in the number of regulatory B cells (Breg) $[117,295]$ and a disruption in the equilibrium between pro- and anti-inflammatory immune cells resulting in disturbed cytokine networks [34, 35].

The function of vitamin $D$ as an immune regulator most probably explains its proposed disease modulating effects. [296] It is well established that immune cells are able to react to and even to metabolize vitamin D. $[105,147,197]$ In vivo association studies with vitamin D, measured as serum 25 -hydroxyvitamin $D(25(\mathrm{OH}) \mathrm{D})$, suggest that vitamin $\mathrm{D}$ can help to maintain or restore the immune homeostasis in auto-immune diseases by promoting the number and/or suppressive function of Tregs. [296] Directly or indirectly via the modulation of the regulatory lymphocytes, vitamin $D$ may inhibit $T$ cell proliferation, affect cytokine production by T cells, and change the balance between T cell subsets. [296] In MS patients, lower pro-inflammatory cytokine levels, including IFN- $\gamma$ levels, produced by peripheral blood mononuclear cells (PBMC) and T cells, were associated with higher serum 25(OH)D levels, 
while anti-inflammatory cytokine production, such as IL-4 and IL-10, was positively associated with serum 25(OH)D levels. [191] Patients with lower serum 25(OH)D levels tended to have a higher proportion of $\mathrm{IL}-17^{+}$Th cells, but $25(\mathrm{OH}) \mathrm{D}$ levels did not correlate with the proportion of Bregs. [122, 191, 295] GM-CSF production by Th cells was differentially inhibited by vitamin $D$ in an in vitro study in MS patients and healthy controls (HC). [297] In HC, in vivo high dose vitamin $\mathrm{D}_{3}$ supplementation studies (most of them uncontrolled and with a maximum follow-up of 12 weeks) have shown a significant increase in the numbers of Tregs without any effect on the function of these Tregs or on other immune cells. $[296,298]$ Others showed that vitamin $\mathrm{D}_{3}$ supplementation in $\mathrm{HC}$ resulted in a reduced frequency of $\mathrm{IL}-17^{+} \mathrm{Th}$ cells and an increase in the production of the regulatory cytokine IL-10 by non-T cells after 3 days of stimulation with PHA. [187] In MS patients, vitamin $D_{3}$ supplementation resulted in a shift in the balance between the proportions of IFN $-\gamma^{+}$and IL $-4^{+}$Th cells and an increase in the amount of inducible Tregs (iTreg) as proportion of total Th cells, whereas the number and function of natural Tregs (nTreg) was not affected. [79] In general, data on vitamin D and the immune system are not consistent and studies were often under-powered, but they do suggest that vitamin $D$ promotes anti-inflammatory/regulatory immune parameters and reduces pro-inflammatory immune parameters.

In this randomised placebo-controlled trial (RCT) in patients with RRMS, we assess the effects of vitamin $D_{3}$ supplementation as add-on therapy to IFN- $\beta$ treatment on the circulating lymphocyte compartment. In line with our hypothesis, i.e. vitamin $D$ being an important player in the immune regulation and Th cells being the most relevant cells of the immunological cascade in the immune cell compartment of patients with MS [14], we first report on the vitamin $D_{3}$ effects on regulatory lymphocytes. Thereafter, the effects on inflammatory cytokine production by PBMC and Th cells are described.

\section{Methods}

\section{Patients}

In- and exclusion criteria and the study design of the SOLAR study are described elsewhere. [136] In short, study participants, aged between 18 and 55 years, had a diagnosis of RRMS (according to McDonald criteria 2005) which was confirmed by typical MS findings on brain or spinal MRI. They had a first clinical event within 5 years prior to screening and active disease in the prior 18 months, but no relapse 30 days before inclusion. Patients were excluded if they took more than $1000 \mathrm{IU}(25 \mu \mathrm{g})$ of vitamin D supplements. All patients received IFN- $\beta$-1a (Rebif $®$, Merck Serono S.A., Darmstadt, Germany), $44 \mu \mathrm{g}$ three times weekly s.c. for a minimum of 90 days and not longer than 18 months. After being randomised, patients received either IFN- $\beta-1 a$ and placebo or IFN- $\beta$ - $1 a$ and vitamin $D_{3}$ (cholecalciferol, Vigantol ${ }^{\circledR}$ Oil, Merck KGaA, Darmstadt, Germany) 7000 IU daily for 4 weeks, followed by 14000 IU daily up to week 48.

Patients for the SOLARIUM sub-study were recruited in four of the five participating centres in the Netherlands (Zuyderland Medical Centre (Sittard), Maasstad Hospital (Rotterdam), 
Groene Hart Hospital (Gouda) and St. Antonius Hospital (Nieuwegein)), without additional inclusion or exclusion criteria. They were eligible when they agreed with participation in this sub-study. The study protocol was approved by the Medical Ethical Committee Zuyderland Zuyd (Heerlen, the Netherlands). Researchers performing the immunological analyses were blinded for treatment and patient details.

\section{PBMC isolation}

Peripheral blood samples from study subjects were collected at baseline (wk0) and after 48 weeks (wk48) of treatment (placebo or vitamin $D_{3}$ ). Blood was collected in a $10 \mathrm{~mL}$ sodium heparin blood sampling tube (BD Bioscience, Breda, the Netherlands) and kept at room temperature during transportation to Maastricht University Medical Centre, the Netherlands. PBMC were isolated within 24 hours using a Ficoll-density gradient (Histopaque; Sigma Aldrich, Zwijndrecht, the Netherlands) and centrifugation, performed as described before. The freshly isolated PBMC were kept in culture medium. [191]

\section{Staining for detection of $T$ and $B$ cell subtypes}

Directly after isolation, PBMC were stained with a cocktail of monoclonal antibodies to identify nTreg according to the following definitions $\mathrm{CD} 25^{+} \mathrm{CD} 127^{-} \mathrm{CD} 4^{+} \mathrm{T}$ cells $\left(\mathrm{CD} 25^{+} \mathrm{CD} 127^{-}\right.$ nTreg), $\mathrm{CD} 25^{+} \mathrm{FoxP}^{+} \mathrm{CD}^{+} \mathrm{T}^{\mathrm{T}}$ cells $\left(\mathrm{CD} 25^{+} \mathrm{FoxP3}^{+} \mathrm{nTreg}\right)$ and $\mathrm{CD} 25^{+} \mathrm{CD} 127^{-} \mathrm{FoxP}^{+} \mathrm{CD}^{+} \mathrm{T}^{+}$ cells $\left(\mathrm{CD} 25^{+} \mathrm{CD} 127^{-} \mathrm{FoxP}^{+} \mathrm{nTreg}\right)$, as described before $[79,122]$ (Figure $\left.1 \mathrm{~A}\right)$. Within the $\mathrm{CD} 25^{+} \mathrm{CD} 127^{-} \mathrm{nTregs}$, subsets were defined based on expression of CD45RA (naïve/memory nTreg) and CD39 $\left(C^{2} 39^{+}\right.$nTreg, which are the Tregs associated with the suppression of the encephalitogenic IL-17 ${ }^{+}$Th cells [121]).

Cytokine expression of IL-4, IFN- $\gamma$, IL-17, IL-22, GM-CSF and TNF- $\alpha$ by CD3 ${ }^{+}$CD8 ${ }^{-}$T lymphocytes, which are further referred to as Th cells [79], was assessed after a 5 hours in vitro activation with phorbol 12-myristate 13 -acetate (PMA) $(50 \mathrm{ng} / \mathrm{mL}$, Sigma Aldrich) and ionomycin (1 $\mu \mathrm{g} / \mathrm{mL}$, Sigma Aldrich) in the presence of monensin $(1.25 \mu \mathrm{g} / \mathrm{mL}, \mathrm{BD}$ Biosciences). Expression of IL-10, which was used to define IL-10 $0^{+}$Th cells/ iTreg, was assessed after similar activation, but without the addition of monensin. [299] Cells were intracellularly stained after fixation and permeabilisation (Cytofix/Cytoperm, BD Biosciences). Antibodies used, are described before [79, 297], except for IL-22 (anti-IL-22-efluor-660 (eBioscience, Vienna, Austria)) and TNF- $\alpha$ (anti-TNF- $\alpha-P E-C y 7$ (eBioscience)). Gating strategies are shown in Figure $1 B$.

To identify Breg, PBMC were stimulated with CpG oligodeoxynucleotide 2006 (0.1 $\mu \mathrm{M}$, Invivogen, Toulouse, France) for 66 hours in a 24 -wells plate. Subsequently, PBMC were restimulated for six hours with PMA $(10 \mathrm{ng} / \mathrm{mL})$, ionomycin $(1 \mu \mathrm{g} / \mathrm{mL})$ and brefeldin A (BFA) (Sigma Aldrich, $1 \mu \mathrm{g} / \mathrm{mL}$ ) and then stained to distinguish IL-10 ${ }^{+} 7 \mathrm{AAD}^{-} \mathrm{CD} 19^{+} \mathrm{CD} 3^{-}$cells (IL- $10^{+} \mathrm{B}$ cells/Breg), as described before [295] (Figure 1B). Furthermore, to identify CD24 ${ }^{\text {high }} \mathrm{CD} 38^{\text {high }}$ $\mathrm{CD} 19^{+} \mathrm{B}$ cells directly ex vivo, anti-CD19-PerCP-Cy5.5 (BD Bioscience), anti-CD24-PE (Biolegend, Uithoorn, the Netherlands) and anti-CD38-APC (BD Bioscience) were used. 

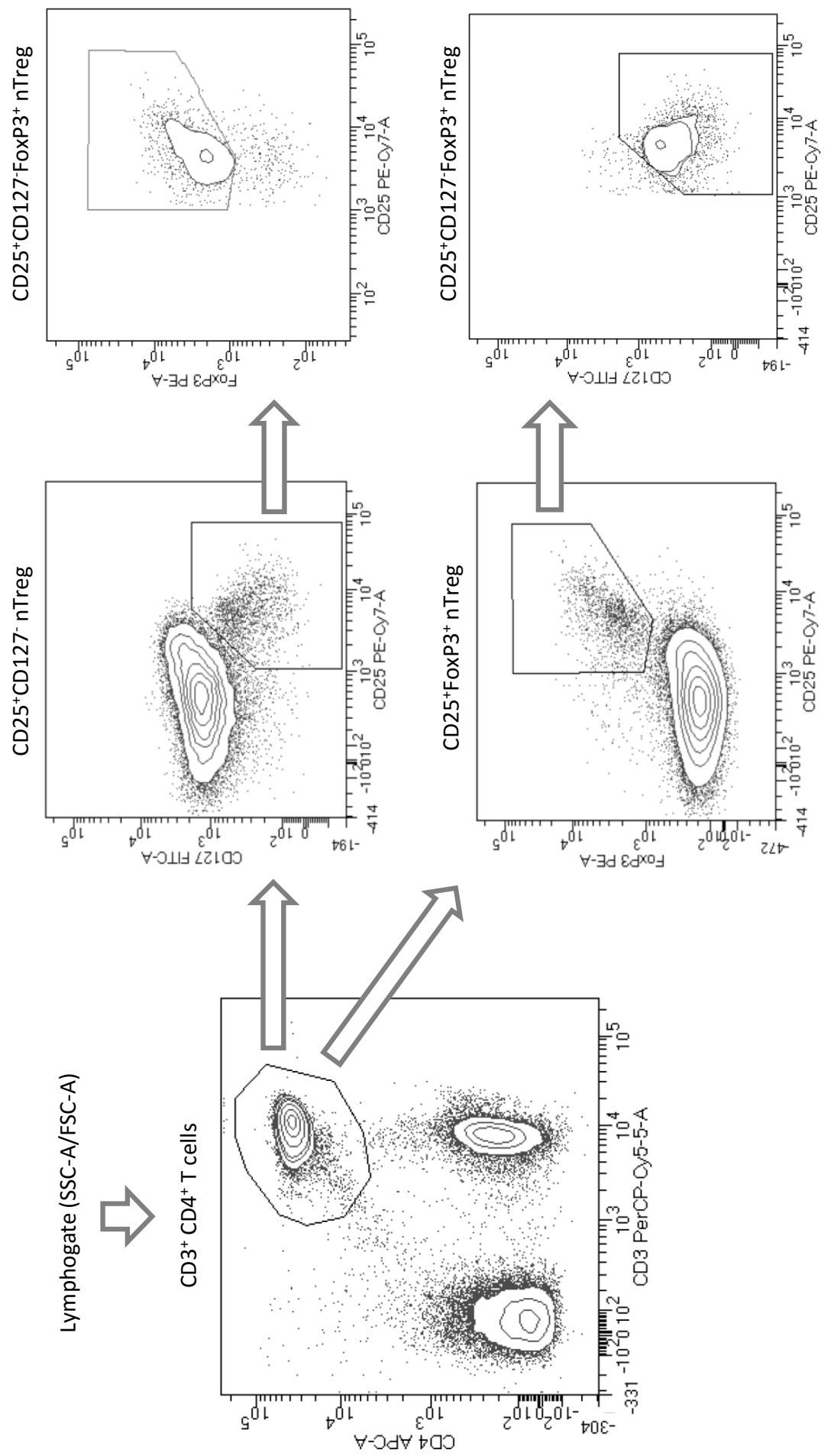

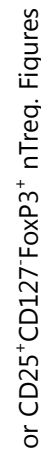

倠

苞

บิ ิㅗ

ㅇำ

ㄱํำ

㐫党

仓ั

$-m$ ह

这

ฮ

華

응 줄

है $\frac{\mathrm{\sigma}}{\mathrm{\varepsilon}}$

Ð

प

는

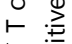

+

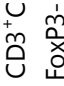

ธำ

离

돈

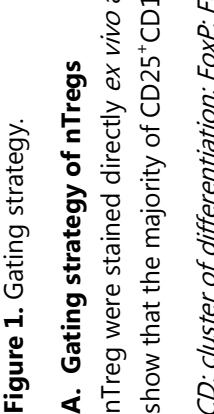



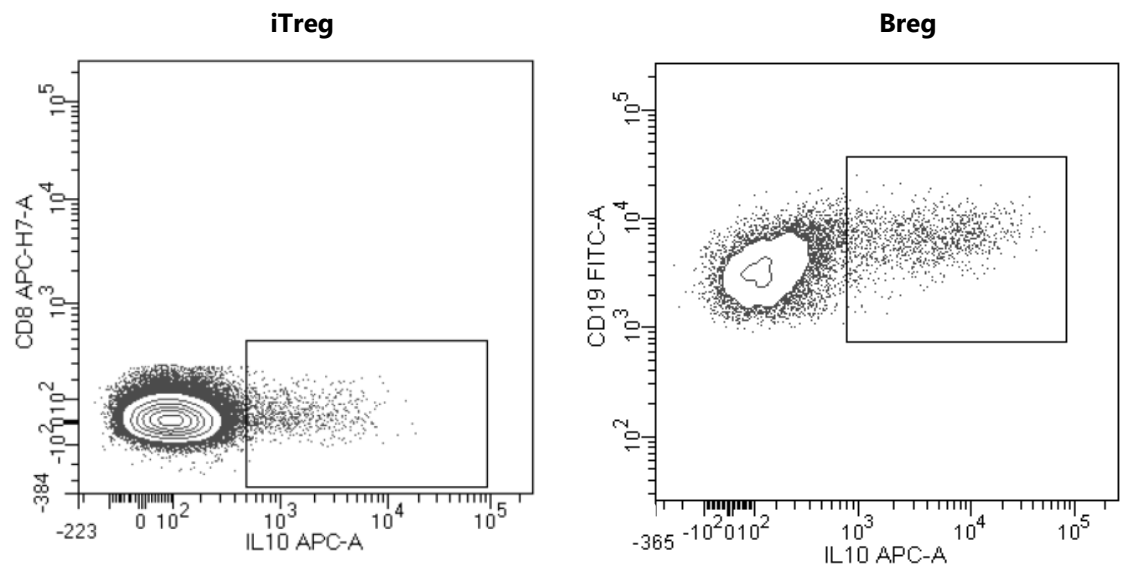

Figure 1. continued.

B. Gating strategy of cytokine production by lymphocyte subsets

$\mathrm{IL}-10^{+}$Th cells/ iTreg were measured as IL- $10^{+} \mathrm{CD} 8^{-} \mathrm{T}$ cells after 5 hours of stimulation with PMA/ionomycin in the absence of monensin. IL- $4^{+}, \mathrm{IFN}-\mathrm{\gamma}^{+}, \mathrm{IL}-17^{+}, \mathrm{GM}-\mathrm{CSF}^{+}, \mathrm{IL}-22^{+}$and TNF- $\alpha^{+}$Th cells were measured after 5 hours of stimulation with PMA/ionomycin in the presence of monensin. Cytokine producing $\mathrm{T}$ cells were gated from the $\mathrm{CD}^{+}$cells within the lymphogate

$\mathrm{IL}-10^{+} \mathrm{B}$ cells/ Breg were measured as IL- $10^{+} \mathrm{CD} 19^{+} \mathrm{CD} 3^{-} \mathrm{B}$ cells within the lymphogate after stimulation for 72 hours with $C P G$ and restimulation for the last 6 hours with PMA/ionomycin/BFA.

BFA: brefeldin A; Breg: regulatory B cell; CD: cluster of differentiation; GM-CSF: granulocyte macrophage colony-stimulating factor; IFN: interferon; IL: interleukin; iTreg: inducible regulatory $T$ cell; PMA: phorbol 12 myristate 13-acetate

After (re)stimulation for intracellular staining, the activation status of cells was assessed by staining cells with anti-CD69-PE-Cy7 (Biolegend). Samples were measured on a FACS Canto II flow cytometer and analysed with FACS Diva software 8.01 (both BD Biosciences).

\section{Cytokine secretion upon T cell activation using Luminex technology}

To investigate the effects of in vivo high dose vitamin $\mathrm{D}_{3}$ supplementation on cytokine production, PBMC were stimulated in vitro for 72 hours with soluble anti-CD3 (WT32, $4 \mathrm{ng} / \mathrm{mL}$, kindly provided by Dr. W. Tax, Nijmegen, the Netherlands) in a 96-wells plate. In addition, we assessed the reactivity of immune cells to vitamin $\mathrm{D}$ at $w \mathrm{k} 0$ and $w k 48$ in vitro. Hereto the active vitamin D metabolite $1,25(\mathrm{OH})_{2} \mathrm{D}_{3}\left(10^{-8} \mathrm{M}\right.$, Sigma Aldrich) was added to the anti-CD3stimulated PBMC. All conditions were cultured in duplo. After 72 hours, culture plates were centrifuged and culture supernatant was collected and stored until further analyses. At the end of the study, an in-house developed and validated multiplex immunoassay (Laboratory of Translational Immunology, University Medical Centre Utrecht, the Netherlands) based on Luminex technology (xMAP, Luminex, Austin TX, USA) [300] was used to assess the amount of produced cytokines (IL-4, IL-5, IL-10, IL-17, IL-22, TNF- $\alpha$, IFN- - , LAP, GM-CSF) in each culture supernatant. Data were analysed using Bio-Plex Manager software, version 6.1.1 (Biorad laboratories, Hercules, CA, USA). 


$$
\mathrm{n}=64
$$

Dutch IFN- $\beta$ treated patients with RRMS included in the SOLAR trial

$$
\mathrm{n}=\mathbf{5 8}
$$

informed consent for participation in SOLARIUM substudy

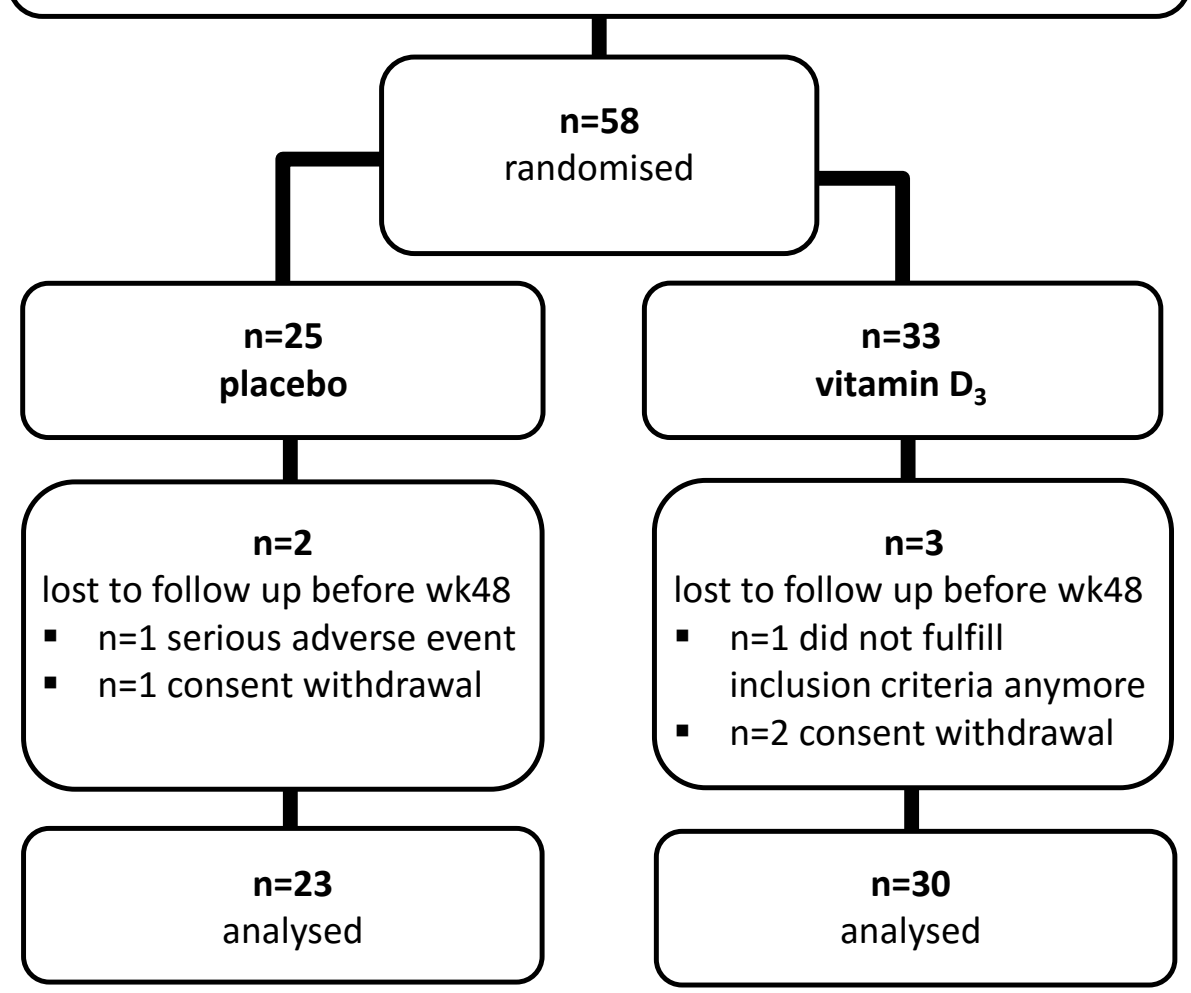

Figure 2. Schematic overview of SOLARIUM study participants' inclusion and analysis. IFN: interferon; $n=$ number 


\section{Additional laboratory parameters}

Analyses of additional laboratory parameters were done by Covance (Princeton, NJ, USA) as part of the SOLAR trial. Serum 25(OH)D (with an upper limit of $250 \mathrm{nmol} / \mathrm{L}$ ) and $1,25(\mathrm{OH})_{2} \mathrm{D}$ levels (upper limit $442 \mathrm{nmol} / \mathrm{L}$ ) were measured (radioimmunoassay, Packard Cobra II Gamma Counter (GMI, Ramsey, MN, USA)). The white blood cell count (WBC) and proportion of lymphocytes were used to calculate the absolute number of $T$ and $B$ cells.

\section{Statistical analyses}

SPSS software (SPSS Inc., version 20.0, Chicago, IL, USA) was used to assess the effect of vitamin $D_{3}$ supplementation on immunological outcome measures. Normality of data was assessed by visual inspection of histograms with normal curves, skewness and kurtosis and normality tests (Kolmogorov-Smirnov and Shapiro-Wilk). For normal distributed populations, means and standard deviations are shown. For non-normal distributed populations, median and range (Q1-Q3) are shown. To test for statistical significant differences between follow-up measurements within each group, a paired T test or Wilcoxon rank test was used as appropriate. ${ }^{10} \mathrm{log}$ ratios ( $\left.w k 48 / w k 0\right)$ were calculated to investigate the treatment effect between groups corrected for baseline measurements and T tests or Mann-Whitney $U$ tests were performed accordingly.

A p-value of $<0.05$ was considered statistically significant

\section{Results}

\section{Patient characteristics}

Between March 2011 and February 2014, 64 patients were included in the SOLAR trial in the Netherlands. A flow diagram of the patient inclusion is shown in Figure 2. Fifty-eight of those patients gave informed consent for the SOLARIUM sub-study. Twenty-five of them were allocated to the placebo group, and 33 to the vitamin $D_{3}$ group. Two patients in the placebo group and 3 in the vitamin $D_{3}$ group were lost-to-follow-up (see Table 1 for patient characteristics). Details on supplementation regimen, treatment effects and side effects will be presented in the definitive SOLAR study report. Paired (before and after) samples of 23 and 30 patients were analysed in the placebo group and in the vitamin $D_{3}$ group, respectively. 

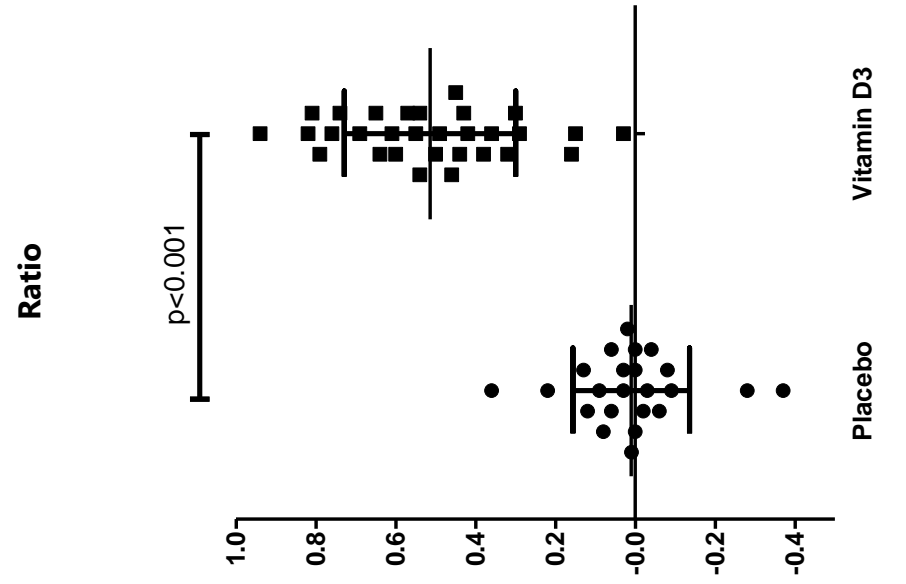

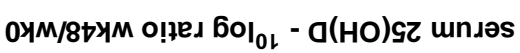

$\frac{a}{\frac{0}{5}}$
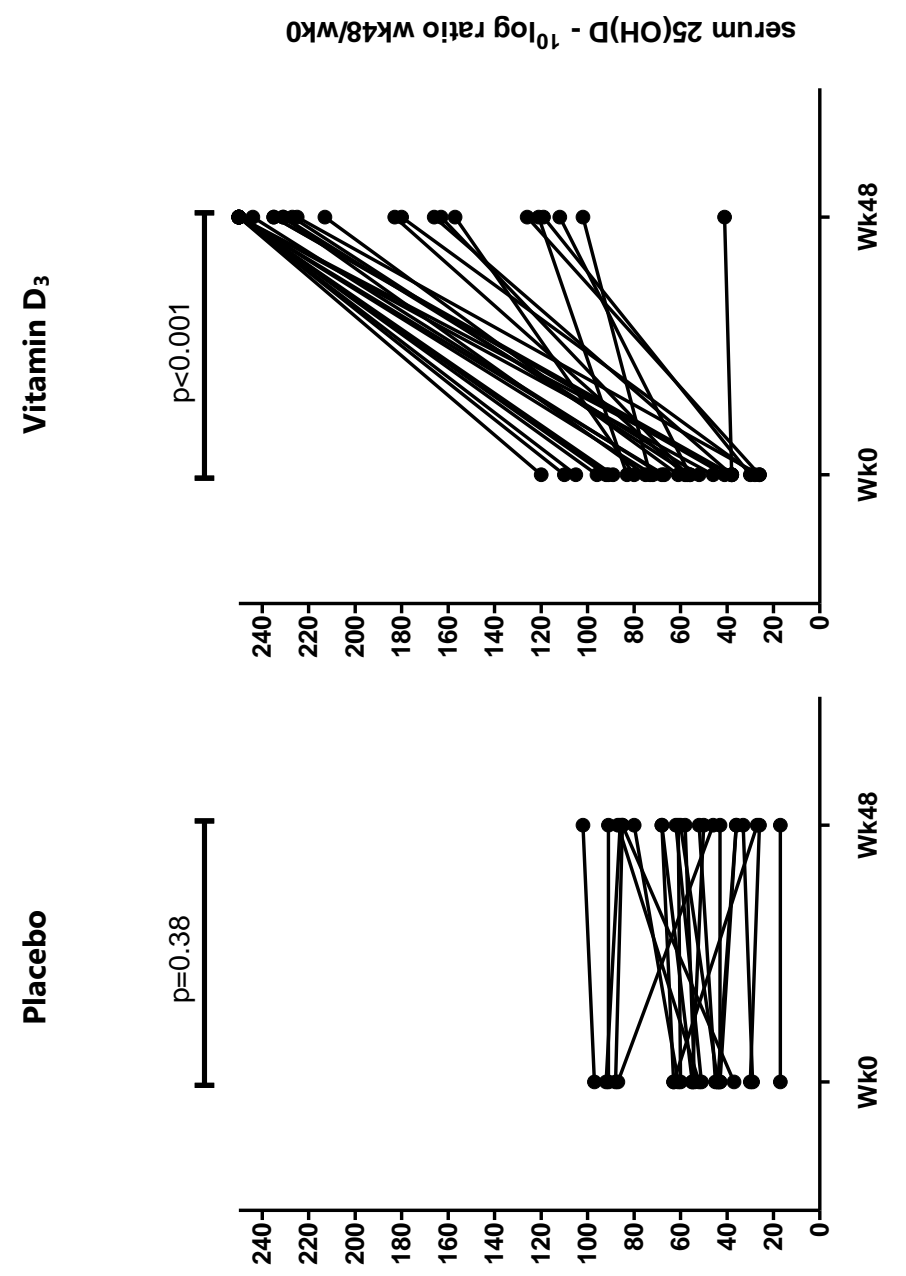

(7/IOUu) a(HO)gz un.ə⿱一土 
Table 1. Baseline characteristics of analysed study participants.

\begin{tabular}{|lll}
\hline & Placebo & Vitamin $\mathbf{D}_{\mathbf{3}}$ \\
\hline Number of patients with RRMS & 23 & 30 \\
\hline Sex (F/M) & $14 / 9(60.9 \% / 39.1 \%)$ & $21 / 9(70 \% / 30 \%)$ \\
\hline Age (years) & $37.2( \pm 9.6)$ & $37.7( \pm 7.2)$ \\
\hline Disease duration (months) & $7.3(4.1-12.3)$ & $7.3(5.0-11.9)$ \\
BMI $\geq 25 \mathrm{~kg} / \mathrm{m}^{2}$ & $13 / 23(56.5 \%)$ & $16 / 30(53.3 \%)$ \\
EDSS at wk0 & & $28(93.3 \%)$ \\
$\quad \leq 3.5$ & $22(95.7 \%)$ & $2(6.6 \%)$ \\
$\quad 4.0-5.5$ & $1(4.3 \%)$ & $1.67( \pm 0.88)$ \\
Number of attacks during past 2 years at wk0 & $1.26( \pm 0.62)$ & $15(50.0 \%)$ \\
$\quad 1$ & $19(82.6 \%)$ & $12(40.0 \%)$ \\
2 & $2(8.7 \%)$ & $3(10.0 \%)$ \\
$\quad \geq 3$ & $2(8.7 \%)$ & $7.4( \pm 3.9)$ \\
\hline Duration since last attack at wk0 (months) & $8.0( \pm 3.1)$ & $6.0( \pm 3.0)$ \\
\hline IFN- $\beta$ duration (in months) & $4.9( \pm 2.5)$ & 28 \\
\hline Patients of Caucasian origin & 23 & \\
\hline
\end{tabular}

BMI: body mass index; EDSS: expanded disability status scale; F: female, IFN: interferon; M: male; RRMS: relapsing remitting multiple sclerosis

\section{Serum 25(OH)D and 1,25(OH) $)_{2} D$ levels are increased in the vitamin $D_{3}$ supplemented group}

Serum $25(\mathrm{OH}) \mathrm{D}$ levels in the placebo group were rather stable $(54$ (43-63) $\mathrm{nmol} / \mathrm{L}$ at wk0 and $60(36-85) \mathrm{nmol} / \mathrm{L}$ at $\mathrm{wk} 48, \mathrm{p}=0.38)$, while they increased significantly from 60 (38-85) $\mathrm{nmol} / \mathrm{L}$ at wk0 to $231(162-250) \mathrm{nmol} / \mathrm{L}$ at wk48 in the vitamin $\mathrm{D}_{3}$ group $(\mathrm{p}<0.001)$. These differences were significant between the groups $(p<0.001)$ (Figure 3). Also $1,25(\mathrm{OH})_{2} \mathrm{D}$ levels increased significantly in the vitamin $D_{3}$ group while they were stable over the follow-up time in the placebo treated patients (data not shown).

Vitamin $D_{3}$ supplementation does not affect the total amount of lymphocytes (subsets) We first assessed the total amount of lymphocytes, which were similar between wk0 and wk48 for both groups (total white blood cells count from $5.9^{*} 10^{9}\left( \pm 2.1^{*} 10^{9}\right.$ ) cells/L to $5.8^{*} 10^{9}$ $\left( \pm 2.4^{\star} 10^{9}\right)$ cells $/ \mathrm{L}$ in the placebo group and from $5.1^{*} 10^{9}\left( \pm 1.8^{\star} 10^{9}\right)$ cells $/ \mathrm{L}$ to $5.1^{\star} 10^{9}$ $\left( \pm 1.7^{*} 10^{9}\right)$ cells $/ \mathrm{L}$ in the vitamin $\mathrm{D}_{3}$ group; $\mathrm{T}$ cells from $1.1^{\star} 10^{9}\left( \pm 0.6^{\star} 10^{9}\right)$ cells/L to $0.9 * 10^{9}$ $\left( \pm 0.6 * 10^{9}\right)$ cells $/ \mathrm{L}$ in the placebo group and from $0.9^{*} 10^{9}\left( \pm 0.4^{*} 10^{9}\right)$ cells $/ \mathrm{L}$ to $1.0^{*} 10^{9}$ $\left( \pm 0.6^{*} 10^{9}\right)$ cells $/ \mathrm{L}$ in the vitamin $D_{3}$ group and $B$ cells from $0.2^{*} 10^{9}\left( \pm 0.1^{*} 10^{9}\right)$ cells $/ \mathrm{L}$ to $0.2 * 10^{9}\left( \pm 0.1 * 10^{9}\right)$ cells $/ \mathrm{L}$ in the placebo group and from $0.2 * 10^{9}\left( \pm 0.1 * 10^{9}\right)$ cells $/ \mathrm{L}$ to $0.2 * 10^{9}$ $\left( \pm 0.2 * 10^{9}\right)$ cells/L in the vitamin $D_{3}$ group). 

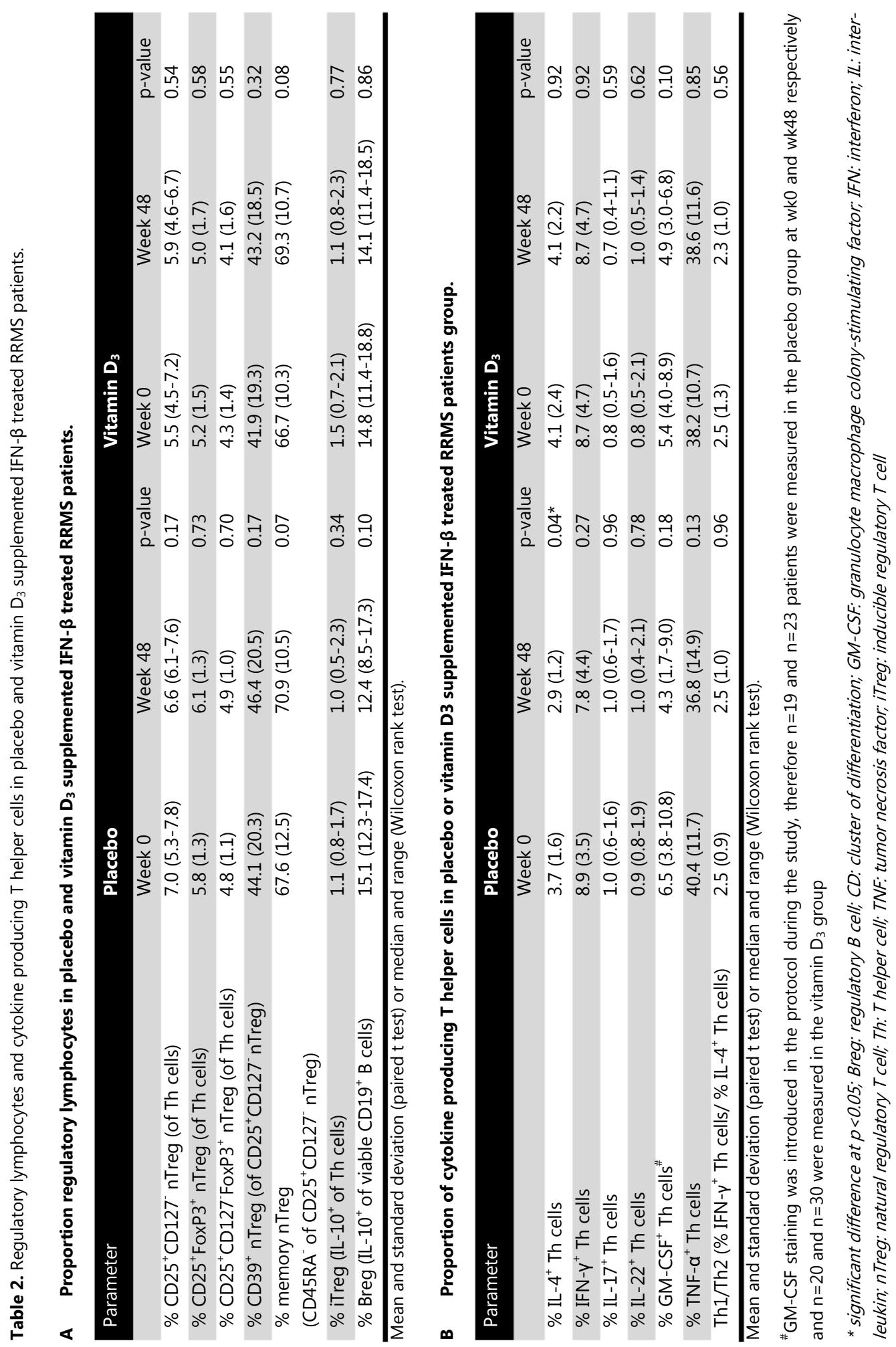


\section{Vitamin $D_{3}$ supplementation does not affect the fraction of Tregs and Bregs}

Next we measured the fraction of Tregs as proportion of $\mathrm{CD}^{+} \mathrm{T}$ cells within the lymphocyte compartment before and after supplementation with placebo or vitamin $D_{3}$. Data are provided in Table 2A and Figure 4. The proportion of nTregs either defined as CD $25^{+} \mathrm{CD} 27^{-} \mathrm{nTreg}$,

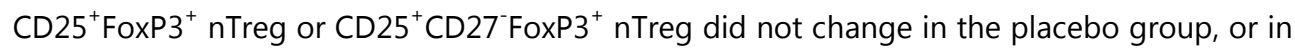
the vitamin $D_{3}$ group. As described previously [79], the different methods to phenotypically characterize nTreg were highly correlated (data not shown). Also the proportion of memory nTregs and $C D 39^{+}$nTregs within the $C D 25^{+} \mathrm{CD} 127^{-}$nTregs, did not change upon vitamin $\mathrm{D}_{3}$ treatment. The proportion of iTregs, defined as IL- $10^{+}$Th cells, was not affected by high dose vitamin $D_{3}$ supplementation and there was no significant difference in the effect between both groups $(p=0.46)$.

Besides Tregs, Bregs may be important for the maintenance of immunological homeostasis and self-tolerance. Also the change in Breg proportions over the follow-up time was not statistically different between the supplementation groups ( $p=0.08$, Table $2 \mathrm{~A}$ and Figure 4). In addition to IL-10 expression in B cells upon in vitro stimulation with $\mathrm{CpG}$, we also used surface markers to identify Breg subsets directly ex vivo. Blair et al. showed that IL-10 ${ }^{+}$Bregs are enriched in the $\mathrm{CD} 24^{\mathrm{hi}} \mathrm{CD} 38^{\mathrm{hi}} \mathrm{CD} 19^{+} \mathrm{B}$ cell subset and that these cells have immune regulatory capacities. [301] We measured these surface markers directly ex vivo in the samples collected at wk48 follow-up. The mean proportion of $\mathrm{CD} 24^{\text {high }} \mathrm{CD} 38^{\text {high }} \mathrm{CD} 19^{+} \mathrm{B}$ cells was $5.2 \%( \pm 4.1)$ in the placebo group and $5.7 \%( \pm 2.9)$ in the vitamin $D_{3}$ group and these proportions did not differ significantly from each other $(p=0.62)$.

\section{Vitamin $D_{3}$ supplementation may balance the $T$ cell compartment in patients with RRMS and prevent a decrease in the fraction of anti-inflammatory cytokine producing Th cells}

Although we did not find any effect of high dose vitamin $D_{3}$ supplementation on the regulatory compartment of the adaptive immune system in RRMS patients, the balance in Th cell subsets may be affected by alternative pathways. Cytokine producing $\mathrm{CD}^{+} \mathrm{T}$ cells can be distinguished in pro-inflammatory (IFN- $\gamma$, IL-17, IL-22, GM-CSF, TNF- $\alpha$ ) and antiinflammatory (IL-4) Th cells. There was no difference in the proportions of pro-inflammatory and potential MS-pathogenic Th cells over the follow-up period of 48 weeks neither in the placebo group, nor in the vitamin $\mathrm{D}_{3}$ group (Table $2 \mathrm{~B}$ and Figure 4 ). The only difference that was observed was a decreased proportion of anti-inflammatory IL- $4^{+}$Th cells over the follow-up period in the placebo group $(3.8 \pm 1.6 \%$ to $2.9 \pm 1.7 \%, p=0.04)$, while the proportion of IL- $4^{+}$Th cells was stable over the follow-up time in the vitamin $D_{3}$ group $(4.1 \pm 2.4 \%$ to $4.1 \pm 2.2 \%, p=0.92)$. Comparing the effect between both groups revealed a significant difference $(p=0.05)$ (Table 2B and Figure 4). However, the ratio IFN $-\gamma^{+}$Th cells/IL-4 ${ }^{+}$Th cells was not influenced by vitamin $D_{3}$ supplementation $(2.5 \pm 0.9$ to $2.5 \pm 1.0(p=0.96)$ in the placebo group and $2.5 \pm 1.3$ to $2.3 \pm 1.0(p=0.56)$ in the vitamin $D_{3}$ group, without a significant difference in effect size between the groups $(p=0.95)$ ). 


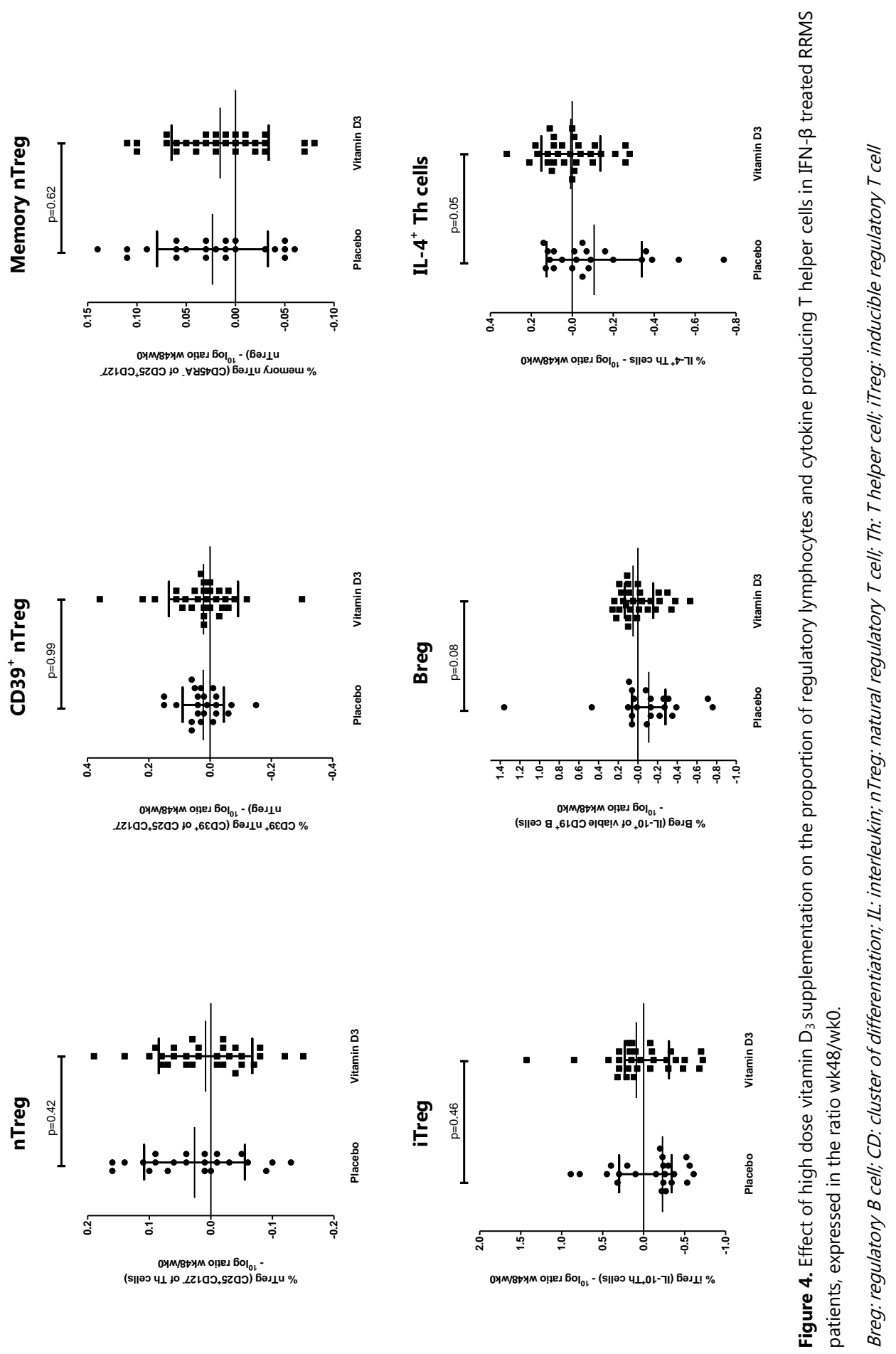



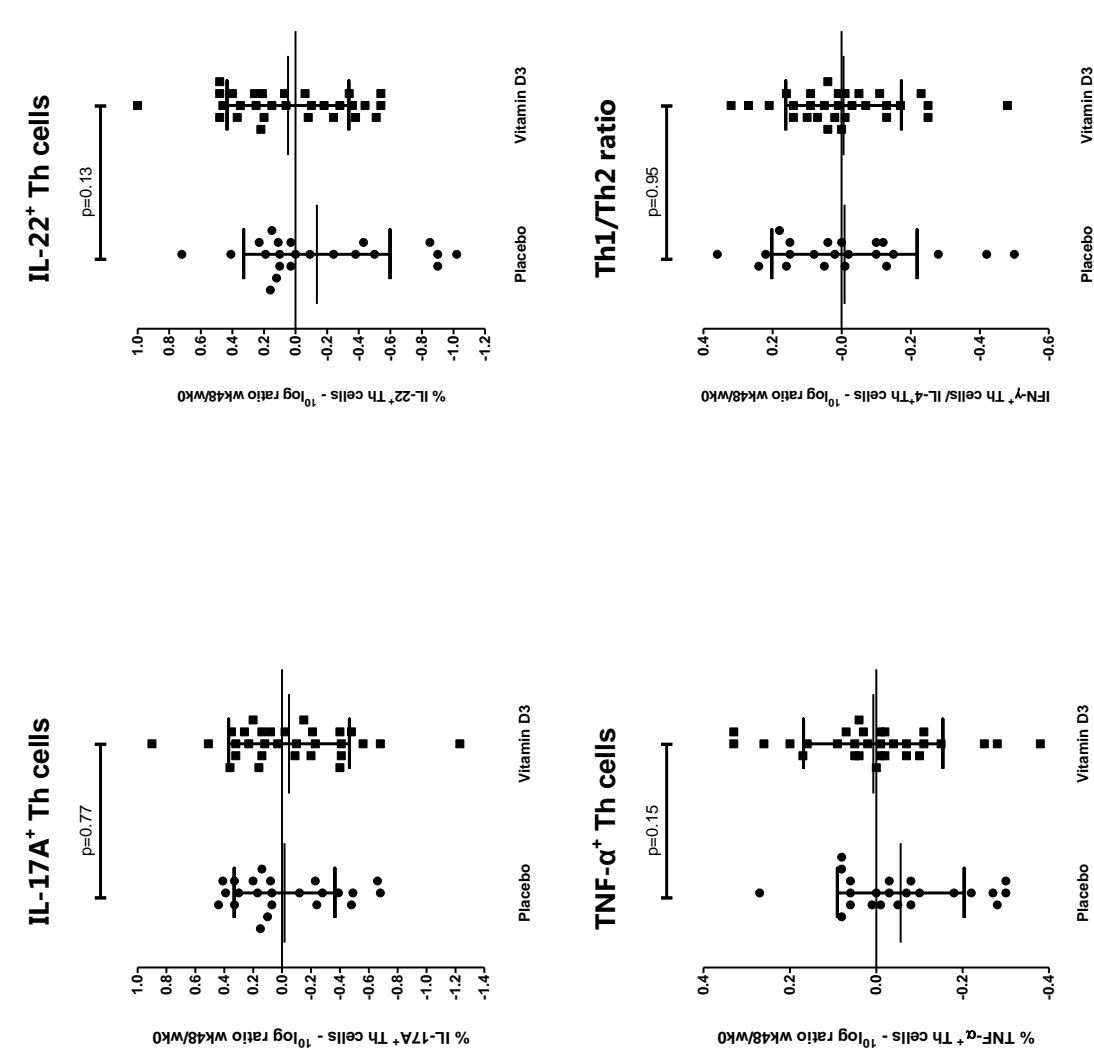

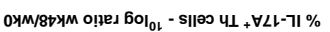
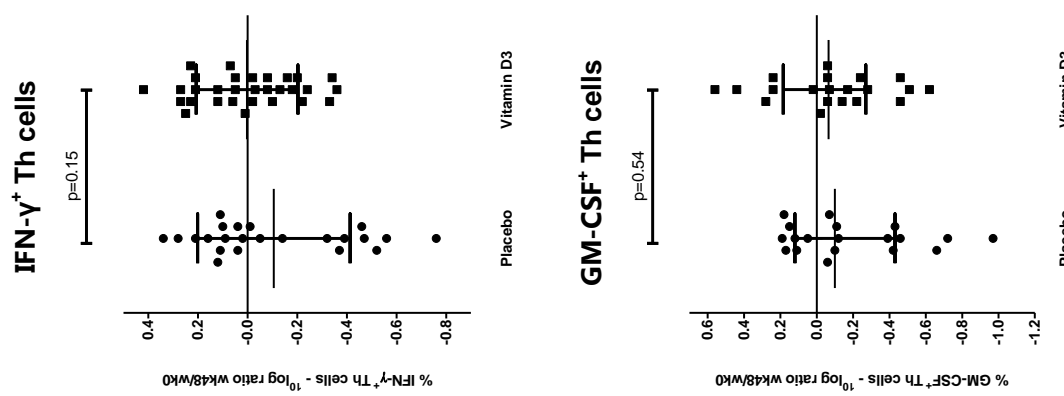


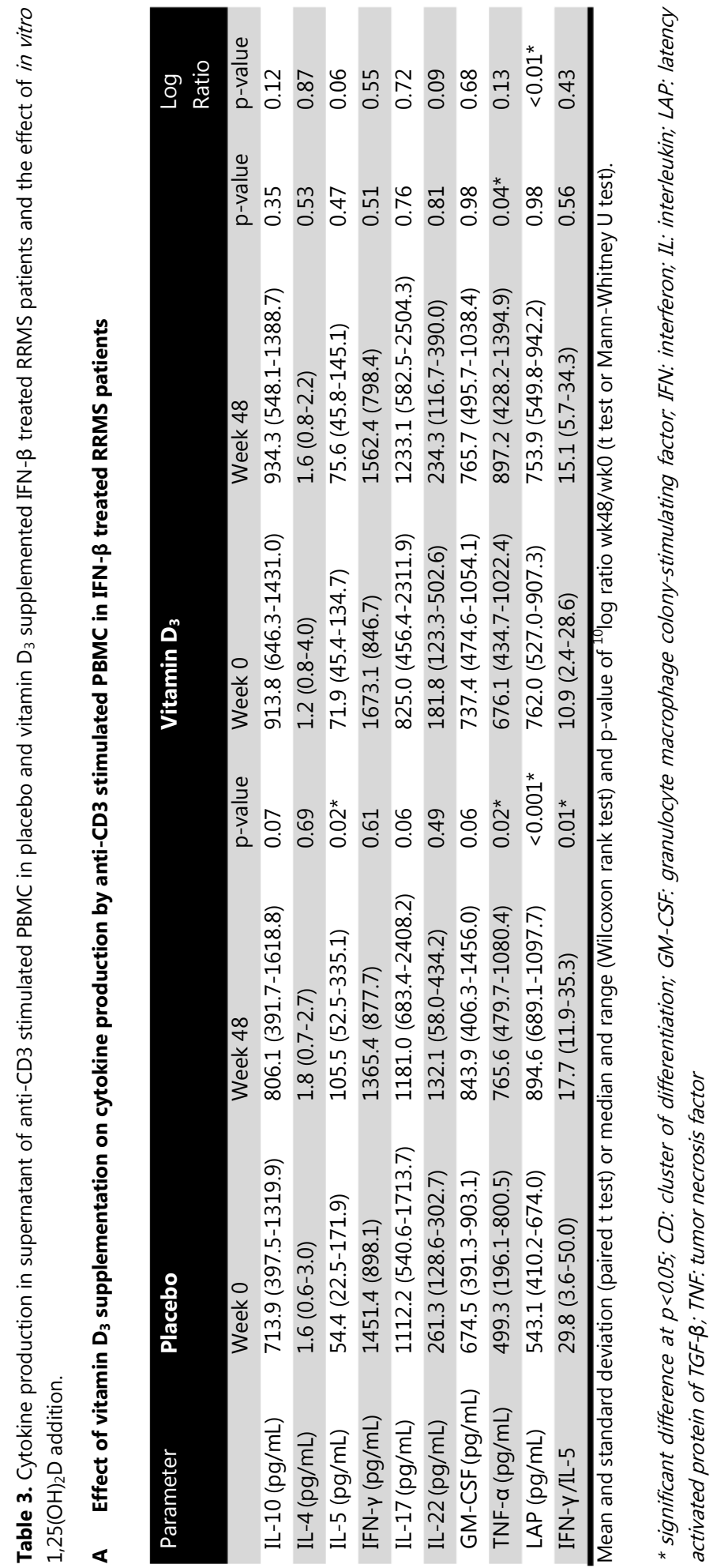


Vitamin $D_{3}$ supplementation prevents an imbalance in cytokine production upon $T$ cell activation but does not change the reactivity to in vitro $1,25(\mathrm{OH})_{2} \mathrm{D}_{3}$

Since we only found minor effects of vitamin $D_{3}$ supplementation on the composition of the T helper cell compartment directly ex vivo, we assessed whether a functional effect on the T cell compartment could be observed. To this end, T cells within PBMC were activated in vitro with anti-CD3 and levels of various cytokines were measured in the culture supernatants. Overall, $T$ cell cytokine secretion increased in the placebo group but not in the vitamin $D_{3}$ group. A significant increase was shown for the anti-inflammatory cytokines IL-5 and latency activated protein of TGF- $\beta$ (LAP) in the placebo group (Table 3A). This was also reflected in a decreased IFN- $\gamma /$ IL- 5 ratio in the placebo group. Likewise, in the placebo group, there was a trend towards increased production of IL-10, IL-17 and GM-CSF over time, which was not seen in the vitamin $D_{3}$ group. TNF- $\alpha$ levels increased in both groups over time. LAP was the only cytokine that significantly increased more in the placebo group then in the vitamin $D_{3}$ group $(p<0.01)$.

To assess whether vitamin $D_{3}$ supplementation also has implications for the responsiveness of lymphocytes to vitamin $D$, we assessed the response of anti-CD3 stimulated PBMC cultures to addition of $1,25(\mathrm{OH})_{2} \mathrm{D}_{3} \cdot 1,25(\mathrm{OH})_{2} \mathrm{D}_{3}$ decreased the amount of the proinflammatory cytokines IFN- $\gamma$, IL-17, IL-22, GM-CSF and TNF- $\alpha$ and increased the anti-CD3 induced amount of the anti-inflammatory cytokines IL-4, IL-5 and LAP in both treatment groups at both time points to the same extent (Table 3B and data not shown). Again, this was also reflected in a decreased IFN- - /L -5 ratio in both groups over the follow-up time. For IL-10 the results were less clear-cut, but there was no difference in effect size between the groups $(p=0.16)$. This indicates that in vitro responsiveness to $1,25(\mathrm{OH})_{2} \mathrm{D}_{3}$ is preserved in lymphocytes after in vivo high dose vitamin $\mathrm{D}_{3}$ supplementation. 


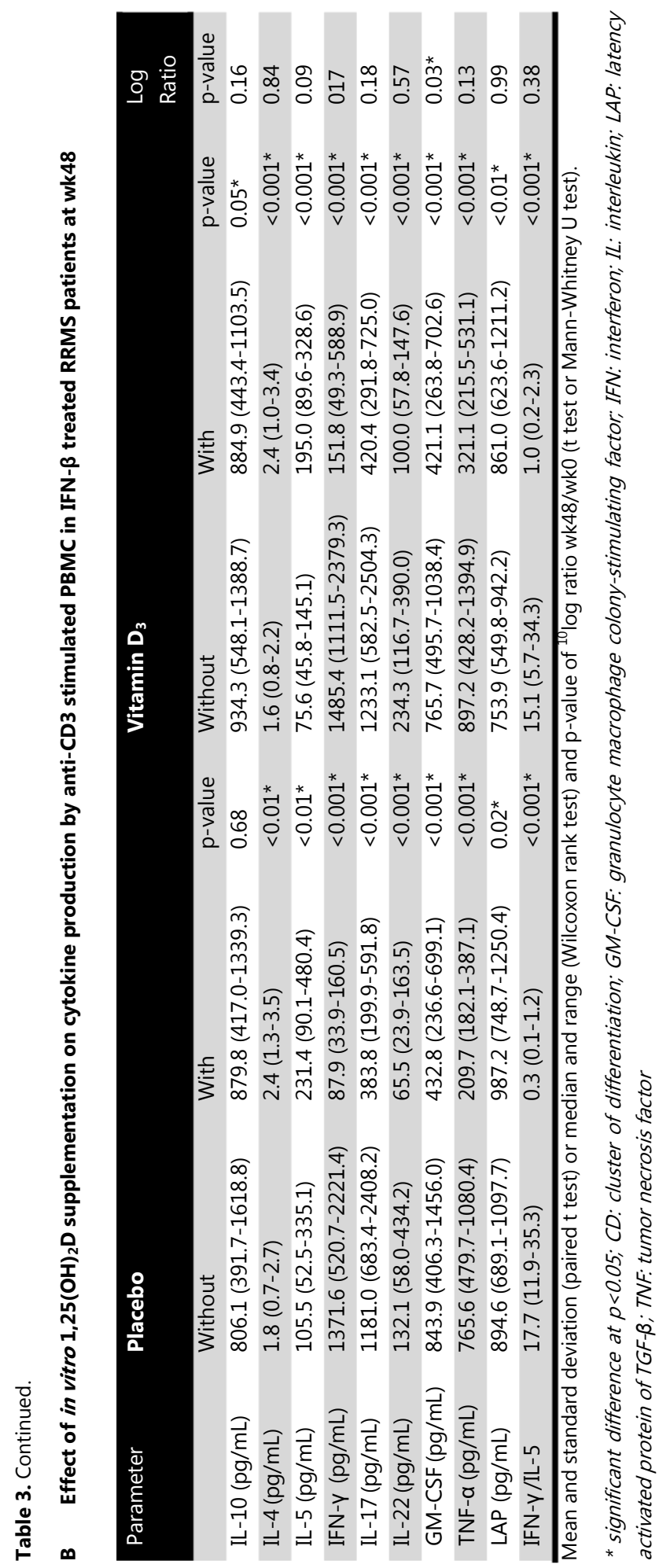




\section{Discussion}

In the last decade, the interest in vitamin $D$ research with respect to several (auto-immune) diseases has increased markedly. In MS, in vitro and in vivo studies have suggested that high dose vitamin $D$ supplementation may be beneficial on clinical and immunological outcome parameters. We investigated the effects of high dose vitamin $D_{3}$ supplementation in a placebo controlled randomised study on relevant immunological parameters in a homogenous patient group with early RRMS that was treated with IFN- $\beta$. The proportion of lymphocytes with a regulatory phenotype did not change over the follow-up after vitamin $D_{3}$ supplementation and there was no difference between the placebo and vitamin $D_{3}$ group. There was also no change in the proportion of Th cells producing pro-inflammatory and pathogenic cytokines at the end of the supplementation period, nor between randomization groups. Although the proportion of Th cells expressing the anti-inflammatory cytokine IL-4 decreased in the placebo group and was stable in the vitamin $D_{3}$ group, this was not reflected in a shift in the ratio of IFN- $\gamma^{+}$Th cells/IL- $4^{+}$Th cells within and between groups.

Despite this minor phenotypic change, functional studies with anti-CD3-stimulated PBMC cultures showed a general trend towards increased levels of cytokines in supernatant over time in the placebo group, most marked of the anti-inflammatory cytokines IL-5 and LAP, while these levels were unaffected in the vitamin $D_{3}$ group. Furthermore, we have shown that the PBMC were still reactive to $1,25(\mathrm{OH})_{2} \mathrm{D}_{3}$ in vitro, independent of in vivo vitamin $\mathrm{D}_{3}$ supplementation. Overall, this study showed that high dose vitamin $D_{3}$ supplementation does not have the proposed effect on the peripheral T cell- and B cell-mediated immune regulation, as has been suggested by the results of previous studies. As a matter of fact, changes are typically observed only in the placebo group. Therefore, it is tempting to speculate that the disturbances in the immune system in the placebo group may reflect disturbances in the adaptive ( $T$ cell) immune response as a possible consequence of ongoing disease activity early in the disease course of RRMS. The enhanced T cell cytokine release is most prominent in the anti-inflammatory immune compartment and this may imply subsequent compensatory mechanisms in early MS. The absence of such disturbances in the vitamin $D_{3}$ group might indicate that vitamin $D_{3}$ can suppress these inflammatory fluctuations by maintaining immune homeostasis and possibly may also be able to suppress subsequent clinical disease activity. Confirmation of this explanation should come from the clinical outcome analyses of high dose vitamin $D_{3}$ supplementation studies, such as the SOLAR trial.

As said, vitamin D research in MS is a hot topic and several RCTs have been performed or are ongoing at this moment, all with different supplementation regimes. Some of the published RCTs analysed immunological parameters. These did not show any effect on serum cytokines in MS, including IL-10 [302-305] and IFN- $\gamma$ [303-305]. The only parameter that increased significantly in the vitamin $D_{3}$ group was circulating LAP/TGF- $\beta$ protein, but whether the effect size was significantly different between the supplemented and the placebo group was not reported. [180, 303] Conflicting and incomplete results have been reported on IL-17. $[303,304,306]$ The change in circulating PBMC mRNA of TNF- $\alpha$, IFN- $\gamma_{\text {, IL-13 }}$ and IL-2 did not 
differ between the placebo and vitamin $D_{3}$ group. [180] In supernatant of PHA stimulated PBMC, IFN- $\gamma$ levels were unaffected whereas TGF- $\beta$ and IL-10 increased and the proliferative response in PBMC of vitamin $D_{3}$ supplemented patients decreased. [186] The effects on $T$ cell homeostasis in $\mathrm{HC}$ were limited to an increase in the proportion of Treg numbers, not function, while none of the proportions of other investigated immune cells (naïve and memory $\mathrm{CD}^{+} \mathrm{T}$ cells, naïve and memory $\mathrm{CD}^{+} \mathrm{T}$ cells, NK cells, NK-T cells, plasmacytoid dendritic cells, myeloid DC, monocytes and neutrophils) were affected. [192, 298] The most recent study in MS, reported a reduced proportion of $\mathrm{IL}^{-17^{+}}$Th cells after high dose vitamin $\mathrm{D}_{3}$ supplementation compared to the group with low-dose vitamin $D_{3}$ supplementation, while effects on Tregs were not reported. [305] Altogether, when reviewing those results, studies so far have mainly reported the positive outcomes, but neglected the negative results, which were actually more apparent.

A decrease in the amount of anti-inflammatory Th cells in RRMS has been described previously. [119] Although we expect anti-inflammatory cytokine producing Th cells to be favourable in MS [307, 308], also anti-inflammatory Th cell cytokines with pathogenic functions have been described $[309,310]$. Our study showed a decreased proportion of IL- $4^{+} \mathrm{Th}$ cells over time in the placebo group, which could suggest that these cells either leave the circulating immune cell compartment and enter the CNS, that they switch to another cytokine producing phenotype [311], that there is a relative increase of other T cell subsets or that they are induced less. Contradictory, in supernatants of PBMC cultures we saw an increase in antiinflammatory cytokines such as IL-4, IL-5 and LAP upon T cell activation. It is possible that these cytokines were produced by other cells, like the $\mathrm{CD} 8^{+}$cytotoxic T cells. [312] In general, we saw a (trend towards an) increase in all pro- and anti-inflammatory cytokines we investigated in the placebo group. Therefore, we speculate that this reflects the dysregulated immune response in the early disease course of RRMS with physiological $25(\mathrm{OH}) \mathrm{D}$ levels, while vitamin $D_{3}$ supplementation towards supra-physiological levels maintains the immunological homeostasis. Although we were not fully able to identify the underlying mechanism from our study, our data point towards a most pronounced difference in anti-inflammatory Th cell proportions and function between the placebo and vitamin $D_{3}$ group. This difference is independent of any effect on proportions of circulating regulatory lymphocytes, although their function was not assessed.

The current study has some limitations. First, we only included IFN- $\beta$ treated patients and therefore cannot extrapolate our findings to other patients with RRMS, who are treatment naïve or are treated with other disease modifying drugs. However, add-on or synergistic effects between vitamin D and IFN- $\beta$ have been described. [223-226] Second, as mentioned above, Treg functionality was not assessed. Previously, we reported a positive correlation between Treg suppressive capacity and vitamin D status in a cross-sectional study. [191] Because we could not detect any improvement of Treg suppressive function after vitamin $D_{3}$ supplementation in our pilot study [79], our current protocol did not include any functional tests on Tregs. Treiber et al. reported an improved Treg suppressive capacity in subjects with diabetes mellitus type 1 after supplementation of vitamin $D_{3}$. [313] We assessed the FoxP3 
MFI in nTregs, which could be an indirect marker of nTreg function. [314] FoxP3 MFI decreased in the placebo group but was stable in the vitamin $D_{3}$ group (data not shown). Although speculative, this may suggest less effective immune regulation in the placebo group, which is in line with the suggested disturbance in immune homeostasis due to disease activity. Lastly, we did not correct for multiple testing. Strengths of this study are that it is a blinded, placebo-controlled study and that we assessed the Th cell compartment as a whole and did not focus on a few selective parameters. Furthermore, we measured paired samples in the same season of one consecutive year, thereby excluding the influence of seasonal effects.

Since the results of our current study reveal only minor effects of vitamin $D_{3}$ supplementation on immune regulation, alternative explanations for the observed associations between vitamin D and MS have to be considered. Firstly, we looked at the immune cell compartment in the circulation which is the only immune compartment easily accessible for analysis in MS patients, whereas the question remains what happens in the secondary lymphoid tissues and targeted brain tissue. [82] Secondly, it might be possible that our supplementation dose was too high or only effective in patients with deficient $25(\mathrm{OH}) \mathrm{D}$ at the start of the supplementation. However, when only the patients with low 25(OH)D levels (below the group median of $56 \mathrm{nmol} / \mathrm{L}$ ) at baseline were assessed ( $\mathrm{n}=27$ (randomization 14/13), 25(OH)D at baseline 43 (30-52) nmol/L), we observed no trends supporting this hypothesis, although numbers were small (data not shown). Thirdly, it has been shown that MS patients have an impaired reaction to vitamin $D[297,315]$ and a lower increase in serum $25(\mathrm{OH}) \mathrm{D}$ after supplementation as compared to HC [316]. This might indicate that the results of vitamin D supplementation in $\mathrm{HC}$ cannot be easily translated to patients with MS. Fourthly, it is possible that vitamin D is 'consumed' by the ongoing inflammatory processes in MS and that low 25(OH)D levels are more a consequence of disease rather than a contributing factor. [317] Supplementation will then not influence the disease activity in ongoing disease. Lastly, it is still possible that it is not vitamin $D$ itself that is the (only) etiologic environmental factor attributing to MS risk and disease severity, but that vitamin $D$ status is a surrogate marker for sun and UV light exposure (also affecting vitamin A [318], melatonin [318-320] and nitric-oxide release [321]) or reflecting patients' general physical and health constitution.

In summary, 48 -weeks of high dose vitamin $D_{3}$ supplementation in IFN- $\beta$ treated RRMS patients does not have substantial beneficial effects on the circulating regulatory immune cell compartment. However, our data point towards a role of in vivo high dose vitamin $D_{3}$ supplementation in the prevention of gradual worsening or (immunological) imbalance, as was shown in the placebo group with a decrease in anti-inflammatory cytokine producing Th cells, and a general (trend towards an) increase in the production of pro- and antiinflammatory cytokines, while these changes were absent in the vitamin $D_{3}$ group. The role of vitamin D in the treatment of MS is therefore much more complex than in vitro, crosssectional, and small high dose supplementation studies have suggested. We showed in this study that vitamin D may prevent the immune system from getting out of control. Since this imbalance has been argued to be most pronounced at the start of disease, vitamin D sup- 
plementation should than be most effective at the start and early phases of the disease course. Whether this also becomes evident in the clinical outcomes, will become clear in the near future. 


\section{Summary and general discussion}




\section{General aim of this thesis}

The increase in vitamin D research during the past decades has raised hopes among many, including patients, clinicians and researchers. The main question now is whether these high expectations can be substantiated by conclusive data or that positive findings from in vitro studies, association studies and small supplementation studies, have hyped those hopes up. In this thesis we focussed on the clinical and immunological effects of vitamin $D$ in multiple sclerosis (MS). We investigated the role of vitamin $D$ in disease progression to enhance our insights in the role of vitamin $D$ in the disease course and studied the immune regulatory effects of high dose vitamin D supplementation as a possible add on treatment in patients with MS.

\section{Summary of results}

In chapter 1, we first reviewed the insights in the in vivo effects on immunological outcomes of vitamin $\mathrm{D}$ in healthy controls $(\mathrm{HC})$, and in patients with an auto-immune disease, at the start of the research described in this thesis. The hypothesized mechanism underlying the association between vitamin $D$ and MS disease activity is the immune regulating capability of vitamin D. In vitro studies and studies in the experimental auto-immune encephalitis (EAE) model had previously shown that immune cells are functional targets of vitamin $\mathrm{D}$, but in vivo studies in humans were heterogeneous and involved multiple immunological outcomes. Overall, there seemed to be a trend towards an anti-inflammatory role of vitamin $D$, but this was not supported by conclusive data. Therefore well powered studies, like RCTs, were needed before definite conclusions could be drawn.

While such trials were executed, in the first part of this thesis, we investigated the role of vitamin $\mathrm{D}$ levels within the physiological range in disease progression, a disability hallmark that was not investigated in great detail so far. This was a relevant question, especially for the treatment of progressive patients, since no treatments have been available to stop the progression of disease. Vitamin D could possibly fill this gap by having a direct effect in the CNS itself. In chapter 2 we used the expanded disability status scale (EDSS) score to investigate the relationship between vitamin $D$ levels and disability progression over a 3 year follow up. Although we could confirm the known relation of a poor vitamin D status with an increased relapse risk in younger MS patients, no statistically significant association was found with disability or disability progression in patients with either relapsing remitting or progressive MS. An alternative way to investigate disease progression is described in chapter $\mathbf{3}$. Here, we investigated the role of vitamin $D$ in the (time to) transition to secondary progressive (SP)MS in patients with relapsing remitting (RR)MS. We could confirm that the patients with SPMS in our study cohort had lower serum 25(OH)D levels when compared to the patients with RRMS. Cross sectional deseasonalised serum 25(OH)D levels in patients with RRMS did not predict the 3 year-risk of conversion to SPMS, but vitamin D levels at diagnosis were significantly lower in patients with RRMS and a very rapid progression to SPMS 
(median RRMS duration of 3.5 (1.0-5.7) years) when compared to matched RRMS patients with a longer RRMS duration (minimum median RRMS duration of 7.7 (6.3-10.0) years, $\mathrm{p}<0.01$ ). Long term follow up of high dose vitamin $\mathrm{D}$ supplementation studies is warranted, to confirm the findings in both these studies on the relation between vitamin $D$ and disease progression.

In the second part of this thesis, the vitamin D effects on immunological outcomes are described. The most recent Th cell, that has been discovered to have strong encephalitogenic capacities in the EAE animal model, is the GM-CSF producing Th cell. Although a clinical trial in patients with MS has been initiated (NCT01517282), data on GM-CSF producing T cells in humans are limited. Therefore, we assessed the role of GM-CSF producing $T$ cells in patients with MS and the mechanisms that might regulate them in chapter $\mathbf{4}$. We showed that the proportion of GM-CSF producing T cells in the circulating immune cell compartment was similar in patients with MS and HC. Furthermore, GM-CSF producing Th cells formed a significant part (39\%) of the total T cell fraction in CSF of both patients with MS and non-MS controls. These results did therefore not support a specific pathogenic role of these cells in MS. In vitro, GM-CSF production by peripheral blood Th cells could be controlled by regulatory $T$ cells and by vitamin $D$. The regulation by vitamin $D$ was less effective in patients with MS.

The most important part of this thesis focuses on the immune regulatory effects of high dose vitamin D supplementation in a randomised placebo controlled trial in patients with RRMS. We expected vitamin $D$ to have a function in the immune regulation, in which the cytokine IL-10 plays an important role. The detection of IL-10 producing Th cells has, however, been reported to be difficult. Appropriate detection of IL-10 is highly relevant for a correct interpretation of the vitamin $D$ effects on immune regulation. In chapter $\mathbf{5}$ we describe an improved method to detect IL-10 producing Th cells using flow cytometry. We showed that omitting monensin, a protein transport inhibitor, from the stimulation protocol, increased the detection of IL-10 producing Th cells, NK-T cells and monocytes, but not B cells.

Chapter 6 reveals the results of the SOLARIUM study. We showed that high dose vitamin D supplementation did not have any substantial effects on the number of circulating pathogenic and regulatory lymphocytes relevant for MS. The anti-inflammatory, IL-4 producing, Th cells decreased over time in the placebo group and were stable in the vitamin D supplemented group, but this was not reflected by a change in the IFN- $-/ \mathrm{IL}-4$ ratio. Functional analyses of supernatant of PBMC upon T cell stimulation, showed an increase of cytokine production in the placebo group, most outstanding in the anti-inflammatory cytokine compartment, while the cytokine expression was stable over time in the vitamin D supplemented group. Altogether, this could point towards a role of in vivo high dose vitamin $D_{3}$ supplementation in the maintenance of homeostasis of the adaptive immune cell compartment during the early RRMS disease course. Importantly, lymphocytes were still reactive to in vitro $1,25(\mathrm{OH})_{2} \mathrm{D}$, irrespective of randomisation group. Whether this balancing role also becomes evident in the clinical outcomes will become clear in the near future. 


\section{Vitamin D research in auto-immunity and MS, is the star on the horizon still shining?}

Vitamin D was discovered as the active anti-rachitic substance in liver oil in 1922 [322] but is mainly obtained via UVB exposure of the skin [323]. Later, in 1960, MS was related to sun exposure [324] and in 1974, the hypothesis of vitamin D being necessary for optimal formation of myelin was put forward. [101] Since then, many researchers, i.e. immunologists, chemists, epidemiologists, geneticists and neurologists, have tried to get a better insight in the true role of vitamin D in auto-immunity and MS. In 2008, our group at the Academic MS Centre Limburg/Maastricht University Medical Centre, jumped into this research line and started with an overview of the then available evidence of the potential immune modulating effect of vitamin D in MS. [131] Some years later, we reviewed the evidence for in vivo effects of vitamin $D$ in the healthy situation and in auto-immunity and concluded that this evidence was inconclusive (chapter $\mathbf{1}$ ). We were not the only ones discussing the role of vitamin $D$ and the potential usefulness of high dose vitamin $D$ supplementation therapies. Vitamin $D$ workshops, originally organized for basic aspects of vitamin D biochemistry and physiology, started to broaden their view and discussions about the pros and cons of vitamin $D$ therapies were hold. [325-328] Commercial companies started advertising campaigns to enhance the purchase of vitamin D supplementation products thereby making also layman aware of the potential beneficial role of this sunshine vitamin, beyond the scope of bone strength. However, this explosion of quantitative interest in vitamin D made the differentiation between firm conclusions drawn from solid scientific experiments and to-be-proven hypotheses drawn from exploratory scientific studies, unclear.

\section{The clinical point of view}

The association between low vitamin $D$ levels and relapse risk in patients with MS, has been substantiated by several studies from different research groups. $[71,72,74,75,103]$ However, more and more recent evidence suggest the strongest relation of vitamin $D$ in the MS disease process in the early (inflammatory) phase of the disease, with the strongest association between low vitamin $D$ levels and relapse risk in MS patients with a short disease duration [103] and in CIS patients [206, 329]. Overall, the studies on clinical outcomes in this thesis confirm this line of thoughts and increase the knowledge on vitamin D and its role in progression of disease. Disease progression is a highly relevant outcome measure as MS is a chronic disease with a major impact on an individual's quality of life and with subsequent consequences for the society. We, indeed, confirmed the relation of vitamin $D$ with relapse rate especially in young patients with MS with a possible role of a low vitamin $D$ at the start of RRMS disease as risk factor for early conversion to SPMS. However, later during the disease course, we did not find a relation between serum vitamin D levels and conversion to SPMS or progression of disease, neither in RRMS nor in progressive MS (chapter 2 and 3). Also other follow-up studies have shown that physiological vitamin D levels in (very) early RRMS and CIS patients were related to MRI outcomes [206, 234] and EDSS [206]. This was, 
however, not the case in established MS where there was no relation between EDSS progression and vitamin D levels. $[73,215,234]$ The dilemma in studying disease progression is that it is not a parameter easy to measure. Disability related outcome measures are most commonly evaluated by using the EDSS score (as mentioned in the general introduction of this thesis). This measure is not ideal, but arguably the best measure available. Besides, it is important that the follow-up period in studies on progression is long enough to measure an effect, keeping in mind the role of other more substantial influencing factors than vitamin $D$, like the immune modulating treatments.

Overall, our and other studies on the role of vitamin D in MS disease progression, suggest that the intake of (high dose) vitamin D supplementation in patients with MS later in the disease course will not affect the progression of the disease. Adequate vitamin D levels might be most relevant for disease activity and disease progression in the very beginning or even before the start of the disease when the inflammatory component is most obvious and maintenance of a (immunological) balance is still possible. This implies a narrow window of opportunity for vitamin D supplementation studies. Leray et al. have postulated that disability progression in MS may follow a two stage pattern: early progression till an irreversible EDSS of 3 and later progression till an irreversible EDSS of 6 . They suggest the progression in the late stage to be independent of the duration and progression in the early phase, both in patients with relapsing remitting and progressive MS. Only in the early phase the number of relapses was an independent predictor of disability progression. [230] If vitamin D would be able to increase the duration of this early phase, this would mean that the onset of the second phase progression, which is probably not caused by focal inflammation, but by diffuse inflammation and neurodegeneration, could be postponed. Besides, this would have important implications for decisions on the continuation of DMTs in the SPMS phase. Currently, most treatments are especially effective in the inflammatory phase of $M S$, meaning that guidelines advice to stop treating patients who enter a secondary progressive phase, especially if they do not experience relapses anymore. [51, 330] However, the decision to discontinue treatment is often difficult to accept for patients and many neurologists continue to prescribe DMTs to patients with SPMS. [331] A postponement of the conversion to SPMS by adequate vitamin $D$ levels early in the disease course could therefore supposedly also increase the window of opportunity for DMTs.

Although one supplementation study showed a trend towards a relation between vitamin $D$ levels and EDSS in RRMS after 1 year [80], long term follow-up of high dose supplementation trials should be able to give definitive answers to the question of the role of vitamin $D$ in MS disease progression. 


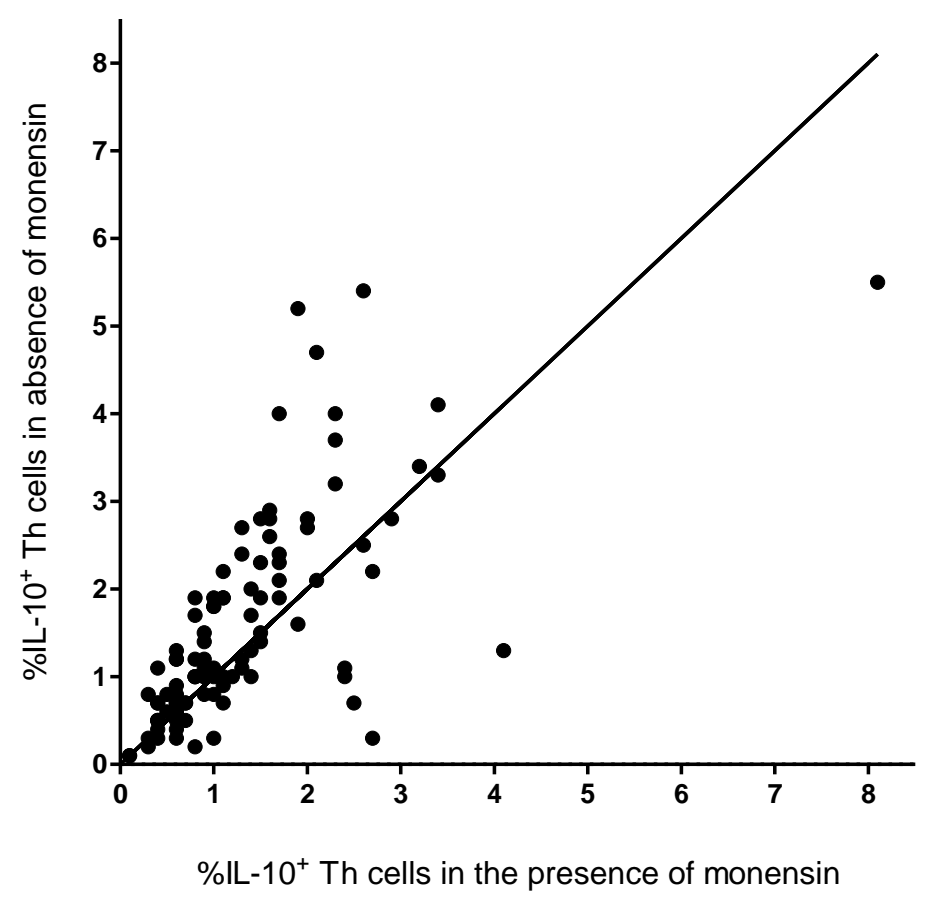

Figure 1. Proportion of IL- $10^{+}$Th cells detected in patients included in the SOLARIUM study at wk0 and wk48, in the presence and in the absence of monensin. The line represents the $y=x$.

\section{IL: interleukin; Th: T-helper}

\section{The immunological point of view}

In the second part of this thesis, we assessed the mechanism underlying the associations between vitamin $D$ and MS disease activity and investigated the effect on relevant immunological outcomes. A recent addition to the panel of T-cell cytokines, suggested to be detrimental in $\mathrm{EAE}$, is GM-CSF. Its role and relevance in MS has not yet been fully consolidated, but it might be a relevant marker to evaluate an immune modulating effect of vitamin $D$ in MS. We therefore first looked at the GM-CSF producing $\left(\mathrm{GM}-\mathrm{CSF}^{+}\right)$Th cells in patients with MS in a cross sectional design, and ascertained that GM-CSF ${ }^{+}$Th cells are not increased in the peripheral blood of patients with RRMS in remission nor in the CSF (chapter 4). In succession of this finding, Noster et al. found that the number of $\mathrm{GM}^{-\mathrm{CSF}^{+}}$Th cells was also not increased in patients with active MS disease, contrary to the numbers in CSF, which they found to be increased compared to non-MS controls. [39] On the opposite, Hartmann et al. reported a borderline significant increased fraction of these cells in the circulating immune cell compartment of patients with MS compared to patients with other neurological diseas-

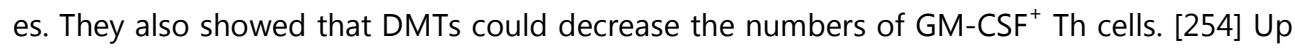
to now, it is still the question whether the pathogenicity of the GM-CSF${ }^{+}$Th cell holds for the whole group of GM-CSF${ }^{+}$Th cells or only for a subset. [39] We showed that GM-CSF ${ }^{+}$Th cells 
were sensitive to regulation by regulatory cells and vitamin $D$, but the in vitro inhibition by vitamin D was less pronounced in patients with MS than in HC (chapter 4). Possible mechanism for this may lay in the genetic differences in vitamin $D$ metabolism or vitamin $D$ response elements in patients with MS, or in a different composition of the gut microbiome. More details on this topic will be discussed in the next paragraph.

Next we assessed the immunological effects in our high dose vitamin D supplementation study in patients with early RRMS. Therefore, we first improved the detection of $\mathrm{IL}-10^{+} \mathrm{Th}$ cells, the so called iTregs, which are important cells for immune regulation and a potential target of vitamin D therapy. The new protocol is described in chapter $\mathbf{5}$. We used this optimized protocol to assess iTregs in the SOLARIUM study were we could confirm our previous findings, i.e. we detected increased proportions of IL- $10^{+}$Th cells after omitting monensin from the stimulation protocol (median proportion of $\mathrm{IL}_{-10^{+}}$Th cell in the presence of monensin $1.0 \%(0.6-1.7)$, in the absence of monensin $1.2 \%(0.7-2.2) ; p<0.001)$ (Figure 1 , unpublished data).

Previously, we [79] and others [180, 186, 192, 302, 303, 306, 320, 332] have reported positive outcomes of vitamin D supplementation on a diverse array of immune parameters. In the SOLARIUM study, we could only detect minor changes on measures of regulatory lymphocyte subset frequencies and lymphocyte cytokine production in the circulating immune cell compartment of the RRMS patient who received placebo, while the measures in the vitamin D group were rather stable over time. The added value of the SOLARIUM study compared to previous studies was that this was a placebo controlled study with relevant power, and a high dose (14.000 IU/day) and long term (48 weeks) vitamin $D_{3}$ supplementation intervention. Results indicate that vitamin $D$ balances the disturbed adaptive immune system during the disease course as was observed in the placebo group. In this group, the proportion of anti-inflammatory IL- $4^{+}$Th cells decreased while a general increase in the amounts of cytokines produced upon T cell activation was shown, most pronounced in the anti-inflammatory compartment. This might point towards a disturbed immune response with accompanying compensatory mechanisms early in the MS course. Vitamin D might hereby be able to better maintain immune homeostasis and consequently control the disease course of MS. These findings are subtle and not entirely in line with earlier cross-sectional findings on immunological outcomes, and their relevance for clinical MS outcomes still needs to be shown. Notably, an effect of vitamin D on the immune pathology of MS is not necessarily the only driver of the numerous associations between vitamin D status and evolution of disease course in MS. Possible alternative hypotheses are plentiful, but cluster around two main themes in which vitamin D either does or does not affect the pathophysiology of MS. 


\section{Food for thought - alternative hypotheses}

\section{Our studies in the context of vitamin D as an effector in the MS pathophysiology}

When assuming that vitamin D does interfere with MS, particularly in the early or pre-clinical phase, our clinical studies confirm this hypothesis and the SOLARIUM study hints towards a more complex mechanistic role of vitamin D within the human body then in vivo animal and in vitro human studies have suggested. These studies showed clear inhibiting effects of vitamin D supplementation on the pro-inflammatory cells of the adaptive immune system, while regulatory lymphocytes and anti-inflammatory lymphocytes were promoted. [131] In the human body, compensatory mechanisms of the neuro-endocrine-immune system, including other vitamins and (sex) hormones, may take place. These interactions may be too complex to observe interference with a single component of this system, like vitamin $D$, in the immune system as a whole or on broad clinical measures. Complex multidimensional measurements, with multiple parameters at the single cell level like those obtained in the SOLARIUM study, make analyses of the right parameters a challenging one. Attempts to translate such datasets into one relevant integrated immune parameter have already been made [333], but might need further investigation.

Although we aimed to look at relevant effects of vitamin D, by taking the regulatory lymphocytes and cytokine producing Th cells as a read-out, measuring vitamin D effects will be dependent on the right location, the right timing and the investigation of the right target cells. The cells we isolated from blood represent a heterogeneous pool of lymphocytes. In contrast, in vitro experiments and in vivo models of neuroinflammation show a more homogeneous pool of (antigen-specific) activated cells, in which vitamin D may interfere. Therefore, an effect of vitamin D on pathogenic T cells in MS may be diluted and not observed in our studies. Another option is that our hypothesis, being vitamin $D$ an immune regulator, was incorrect. Natalizumab, an effective MS drug, has for example been shown to lead to similar or even higher levels of pathogenic IL $-17^{+}$Th cells in the CNS, while one would expect pathogenic Th cells to decrease in number after treatment with an effective drug. [334, 335] This also illustrates that it is possibly not (only) the number of cells that counts but also the functionality of these cells. We did not include the measurement of functional regulating capacity of regulatory lymphocytes in vitro as an outcome measure in our RCT. Our data do not suggest a dramatic shift in markers of immune regulatory cells, but a recent trial showed an improvement of Treg function after supplementation with vitamin $D$ in diabetes mellitus type 1 patients. [313] Other, newly discovered cells that could be relevant as detrimental cells in MS and as targets for vitamin D therapy are specific subsets of GM-CSF producing Th cells $\left(\mathrm{GM}-\mathrm{CSF}^{+} \mathrm{IFN}-\gamma^{+}\right.$double producers or GM-CSF-only producers) [39], IL-2 receptor (CD25) expressing Th cells [336], CD52 Tregs [337] and GM-CSF producing B cells [338]. Furthermore, we did not investigate the direct effect of vitamin $D$ supplementation on the immune cells in the CNS, on the CNS-cells themselves or on the blood brain barrier, which are all difficult to assess in humans. Likewise, migration of lymphocytes into the CNS would be an interesting outcome measure. A chemokine that might be relevant in this perspective 
is CXCR3, involved in trafficking of potential pathogenic immune cells. This trafficking was impaired upon vitamin D supplementation in the EAE model. [339]

Based on previous studies and our own clinical studies, described in this thesis, we included a group of patients with early RRMS who have the highest chance to benefit from high dose vitamin $D$ supplementation. However, the IFN- $\beta$ treatment they received might have obscured the vitamin $D$ effects. Obliging patients to be treatment naive during the vitamin $D$ supplementation period was, obviously, unethical. Moreover, IFN- $\beta$ was one of the most common MS therapies used at the start of the study. Also, associations between vitamin $D$ status and MS outcomes have been predominantly reported in IFN- $\beta$ treated MS patients $[74,206,234,340]$, suggesting this treatment not to interfere with an underlying mechanism. Furthermore, vitamin $D$ supplementation might be effective only in those patients with very low 25(OH)D levels. Yet, the immune system of the subgroup of patients with a very low $25(\mathrm{OH}) \mathrm{D}$ status at baseline did not show more clear-cut effects of high dose vitamin $\mathrm{D}$ supplementation in our study (chapter 6). This makes it also likely that it is not the change in $25(\mathrm{OH}) \mathrm{D}$ level that counts. In line with this notion, SOLAR was certainly not a dose finding study: too high or too low doses of vitamin $D$ may have been supplemented. In addition, we still do not know whether a certain vitamin $D$ level is equally important for each individual. Multiple genes do affect an individual's vitamin D status. [341, 342] Black people have lower serum vitamin $D$ levels when compared to white people, but their bone metabolism is equal. $[343,344]$ Furthermore, also the main risk allele for MS, HLA-DRB1*1501, is regulated by vitamin D [345] just like the risk alleles TAGAP and IL-2R in Th cells [346]. CYP27B1 and CYP24A1, involved in the metabolism of vitamin D, popped up in GWAS. [14] Therefore supplementation might be most beneficial in a certain subgroup of patients with MS, based on their genetic profile. Unfortunately, our group of included patients did not have sufficient power to perform a stratification for this. Combined results of SOLAR and other vitamin $D_{3}$ RCTs may provide more clearness on this issue.

\section{Our studies in the context of vitamin D not affecting the MS pathophysiology}

The main second theme of alternative hypotheses regarding the association between vitamin $D$ status and MS is that findings on the effects of vitamin $D$ in in vitro studies and in vivo animal studies do not reflect the situation in human MS. In the EAE animal models, the active component of vitamin $\mathrm{D}, 1,25(\mathrm{OH})_{2} \mathrm{D}$ has been shown to be beneficial, mainly in the prevention of disease onset, but also in reducing severity of symptoms and increasing survival. [131] Substantial fewer studies have been performed on vitamin D supplementation in mice. Spach and Hayes showed that vitamin D supplementation before immunization inhibited EAE in female mice. [159] Farias et al. replicated this finding with a reduced EAE severity, when vitamin $D$ supplementation was started at the moment of immunization [347] and mice from vitamin D deprived parents developed a precocious and more severe EAE [348]. Also in vitro studies have contributed to the hypothesis of vitamin $D$ having an immune regulatory role by increasing regulatory cells and anti-inflammatory cells and decreasing pro-inflammatory cells. However, here as well, usually the $1,25(\mathrm{OH})_{2} \mathrm{D}$ metabolite is used. In 
humans, though, 25(OH)D levels are associated with clinical MS outcomes, whereas that is less clear for $1,25(\mathrm{OH})_{2} \mathrm{D}$. Besides, supplementation of $1,25(\mathrm{OH})_{2} \mathrm{D}$ may lead to (unacceptable) hypercalcemia risks, while increasing the $25(\mathrm{OH}) \mathrm{D}$ levels might give the body the opportunity to regulate $1,25(\mathrm{OH})_{2} \mathrm{D}$ levels, thereby diminishing the change to develop side effects. Therefore, vitamin $D$ supplementation in humans is based on supplementation with vitamin $\mathrm{D}_{2}$ or $\mathrm{D}_{3}$.

Discrepancies between in vitro and in vivo human studies may also be explained by quantitative issues in the immune responses studied: the in vitro effects of $1,25(\mathrm{OH})_{2} \mathrm{D}$ are usually assessed under quite extreme stimulating conditions, boosting the immune system to a very strong inflammatory reaction, one that will usually not arise in vivo in the human body. A strong aspect of the SOLARIUM is that functional characteristics of isolated PBMC (as well as their responsiveness to vitamin D) were assessed both directly ex vivo as in vitro. Furthermore, in the isolated environment of in vitro experiments, the dynamics of interactions in the human body are lost and other important parts of the immune system, like germinal centres cannot be easily investigated directly ex vivo. [349]

Another interesting point is that our GM-CSF study and another recent study [315] have suggested immune cells of patients with MS to be less sensitive to regulation by vitamin $D$ when compared to cells of HC (chapter 4). This could be due to differences in the vitamin D metabolism. Previously we have shown that the gene expression of the VDR, CYP27B1 and CYP24A1, was not impaired in PBMC and Th cells of patients with MS compared to HC, suggesting a normal vitamin D response and metabolism in both groups. [350, 351] However, Bhargava et al. showed that MS patients have lower $25(\mathrm{OH}) \mathrm{D}$ levels after vitamin D supplementation with $5000 \mathrm{IU} /$ day for 90 days than HC. [316] If it is really true that the vitamin D metabolism in patients with MS is different from that of controls, these differences may be caused by variances in gut absorption, alternations of gut microbiota or in the polymorphisms in genes related to vitamin D metabolism, which are reported in some, but not all, MS genetic studies. It might therefore be that vitamin $D$ supplementation is most relevant for a subpopulation of patients with MS who have a more 'HC-like' microbiome or genetic profile.

In contrast to our current supplementation study, previous supplementation studies, most of them reporting much clearer effects of vitamin $D$ on immunological and clinical outcome measures, were small and uncontrolled. Thereby introducing higher odds for chance and regression to the mean to interfere with the results. Furthermore, the impression that there is an unintended, but clear, bias of positive data selected and published, cannot be banished. Positive results of vitamin $D$ supplementation have been selectively emphasized reporting changes of single cytokines while the more apparent negative results were just briefly mentioned. [187, 302, 306, 352] Blind analyses of all cytokines or cytokine producing $T$ cells might have given a more general view on the effects of vitamin $D$ on the adaptive immune system. This might have taken away the focus on changes in the pathogenic and regulatory cells in MS, and might have led to different conclusions, as has recently been suggested in Nature. [353, 354] 
Ultimately, vitamin D might be a correlate of the causative factor interfering with MS. It may not be the (only) driving force in the relation between MS and sun exposure. Vitamin $D$ is synthesized in the skin upon UVB exposure, in particular UV light with a wavelength between 280 and $310 \mathrm{~nm}$. Although positive associations with vitamin D and disease severity have been described in the EAE animal model, suppression of EAE appeared to be most eminent at wavelengths of 300-315 $\mathrm{nm}$ and was then independent of vitamin D. [355, 356] Vitamin D levels might therefore be a surrogate marker of sun exposure. Other potential candidates of the relationship between sun exposure and MS onset and disease activity are melatonin [319], vitamin A [318] or nitric oxide [321]. A 'consumopathy' of vitamin D by the activated immune cells could also explain the low vitamin D levels in patients with MS. [317] Low levels of vitamin $D$ are then the consequence of the inflammation rather than a cause and higher serum levels of $25(\mathrm{OH}) \mathrm{D}$ will not dampen the inflammatory component. Lastly, most MS outcomes correlate negatively with sun exposure. [73] Therefore, being less exposed to sunlight and vitamin D may be a consequence rather than a cause of having active MS.

\section{Summary}

Summing up, the studies described in this thesis provide additional support for a role of vitamin $D$ and possibly also vitamin $D$ supplementation in the early (and maybe even preclinical) state of MS. Ultimately, to draw conclusions and chose directions for further research on vitamin D in MS, the results of clinical trials are extremely important. These studies will show whether modulation of vitamin D status will affect disease outcomes in MS. One of these studies is the SOLAR study. Data are not yet available, but will be announced in 2016. If modulation of the vitamin D status indeed affects disease outcomes, the question is whether these effects arise from the limited effects on the immune system or that there is a more important target. If not, other factors interfering with both vitamin D status and MS outcome may be more relevant. Altogether, as will be discussed in the next paragraphs, the star on the horizon is sufficiently shining to warrant further research on the role of vitamin D in MS.

\section{Future prospects}

This thesis describes the research done to discover some missing pieces in the story of vitamin D in MS. Although some questions have been clarified, the final answer is not there yet. As already mentioned, supporting evidence suggest the strongest role of vitamin $D$ very early in the disease course. To enhance our knowledge on the long term effects of vitamin $D$, also on disease progression, it is important to follow up the patients in vitamin D supplementation studies, such as the SOLAR study. Future research will possibly also tell us whether other immune modulating drugs, currently in development, might have even more relevant synergistic effects with vitamin D than IFN- $\beta$ has. Vitamin D will probably not be of help in immune cell depleting therapies or therapies blocking the entrance of the immune cells into the CNS, but a possible role in combination with immune modulation, stem cell transplantation $(\mathrm{SCT})$ or remyelination therapies is not unrealistic. Preventive treatment was beyond the 
scope of this thesis, but other studies on vitamin D have shown also associations and effects of vitamin D in the pre-clinical phase of MS: i.e. CIS $[206,329]$ and optic neuritis patients $[357,358]$. These findings confirm our line of thoughts on a restricted window of opportunity for vitamin D supplementation (very) early in (the pre-clinical phase of) MS. One step further, vitamin $D$ treatment could then also be useful in any pre-clinical phase, in which the disease process already started, but no actual symptoms have appeared. It might even be necessary to move our view more towards the prevention of the development of auto-immune disease in children and young adults. To obtain a healthy bone and calcium metabolism, vitamin D supplementation of 400 IU per day is already common in children and adolescents in the USA. [359] In Europe, this is advised for all children till the age of 4 or 5 and till the age of 19 for every child who is at risk of a low vitamin $D$ level due to a dark skin or a minimal time spent outside. [360-362] The question for the future is then whether vitamin D supplementation should be advised for adolescents all over the (Western) world to prevent the development of MS and possibly other diseases. Furthermore, we will have to investigate what the desired dose of supplementation is for this target group.

To obtain more insights into the mechanism of vitamin D and its role in the MS pathogenesis, our longitudinal samples which were collected in the SOLAR and SOLARIUM study are of great value. There are still a couple of interesting questions that need an answer. To gain more insights in what happens in the vitamin D uptake, transport and metabolism in patients with MS, it might be interesting to measure the vitamin D metabolizing enzymes and associated genes in plasma or lymphocytes. Besides these mechanisms playing a role in the effectiveness of vitamin D treatment in patients with MS, also the existence of neutralizing vitamin $D$ antibodies, which were found in serum of patients with systemic lupus erythematosus, may hamper the potency of vitamin D treatment. [363, 364] Furthermore, one could think of investigating the vitamin D binding protein (DBP). The effects of vitamin D supplementation were cancelled out in EAE mice with increased DBP expression. [365, 366] DBP levels have been reported to be increased in patients with MS [365], although our group could not confirm this [367]. Furthermore the investigation of vitamin D metabolites and the free versus bound fraction of $25(\mathrm{OH}) \mathrm{D}$ could learn us more about the actual availability of the 25(OH)D metabolite in the microenvironment of immune- and CNS cells. [368]

As mentioned before, it is possible to look at other target cells of the immune system that were not investigated so far. Furthermore, it would be compelling to know what the actual effects of vitamin D supplementation are on the target tissue, the CNS. However, human brain tissue usually originates from deceased MS patients with more advanced MS. Those samples will probably not be representative of MS manifestations at the start or during the disease course. Therefore, answers to this question will probably need an animal model, which is genetically linked to human MS, like EAE models in primates such as marmosets. Another open question is what happens in the lymph nodes of patients with MS, in reaction to vitamin D supplementation. It would be very interesting to investigate this in the (superficial) cervical lymph nodes were most probably antigen presentation takes place. Also (changes in) the gut microbiome is a hot topic in auto-immunity research. Furthermore it is 
interesting to look at the relations and interactions of different environmental risk factors in MS such as the combination of vitamin D and EBV [349] and to keep our minds open for other underlying mechanism that could explain the relation between sun exposure and MS like melatonin, vitamin $\mathrm{A}$ and nitric oxide.

Intriguing is the difference between in vitro and in vivo vitamin D supplementation studies. Instead of thinking about explanations, we could also turn this phenomenon to our advantage. By in vitro treatment of autologous immune cells, for example T cells, and replacement of those cells in the human body, we could circumvent the in vivo (compensatory) effects. [347] Research on this topic is already ongoing in the group of B. Roep at the University Medical Centre in Leiden (the Netherlands). They are investigating the possibilities of in vitro vitamin $\mathrm{D}$ treatment of dendritic cells (DC) of diabetes mellitus patients. Once placed back in the body these DC could induce the development of Tregs. [369, 370]

More in general, to enhance the progress in MS and environmental factor research, especially vitamin $D$, it is important that researchers in all fields of auto-immunity are open to each other and work together to disentangle the common denominators in the pathogenesis of auto-immune diseases. It is important that people involved continue to look beyond the own niche, traditionally based on the target organ that is affected. Initiatives to facilitate this have already been undertaken by the Dutch Society of Immunology (Balie debat, 20 Nov 2014). $[371,372]$ Another hot topic in the scientific community is the question how to obtain objective analysis and reproducibility of data, which are translatable to the clinic. $[353,354,373$, 374] To this end, it is important that everybody involved in scientific research, i.e. tutors, researchers, journal editors and reviewers is aware of this challenge and continues to do their best to prevent the realisation and publication of low quality research. Recently, it has been shown that the use of positive and negative words in research papers has increased substantially in the last decades, while the use of neutral words decreased. It might be that scientific findings are sometimes overstated or exaggerated in order to be published. [375] Also the high rate of discontinuation and non-publication of RCTs [376] might lead to waste of research sources, ethical concerns, and affect research and patient care. Therefore, it is important to make the publication of negative results not inferior to the publication of positive results. This will overcome false hopes and hypes and will make it possible to focus research sources on the really relevant topics.

\section{Vitamin D in MS: hype or hope?}

The role of vitamin D in MS has been proposed to be twofold. First of all it is presented as one of the identified environmental risk factors in the development of MS. Second, vitamin D has been related to MS disease activity. The research in this thesis focused on the latter topic. Vitamin D research and papers published on this topic have increased significantly in the last decades. In the previous 5 years the increase in papers on vitamin $D$ appears to be stronger than the increase in the total amount of papers published, confirming the gained interest in vitamin $D$ in the scientific community (Figure 2). 


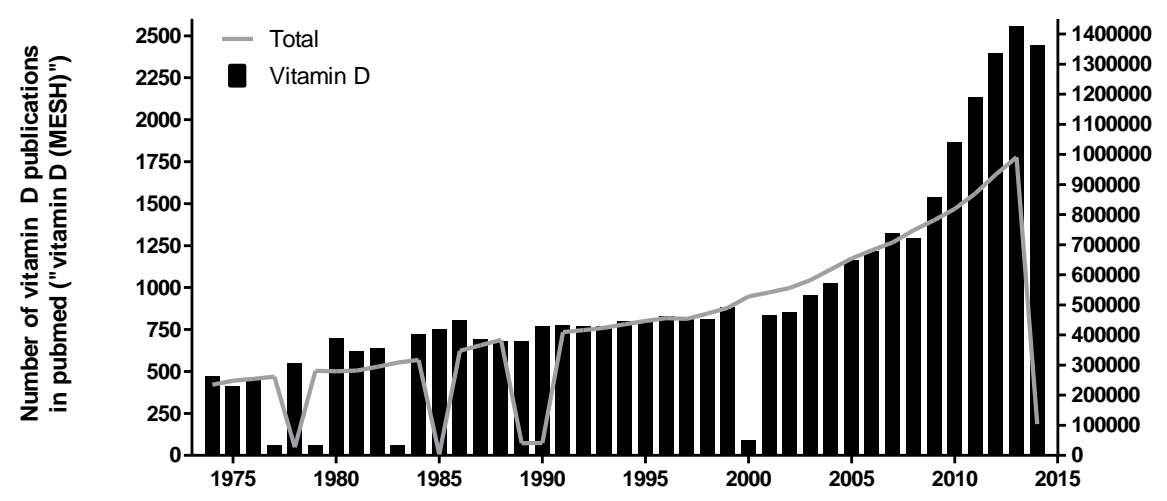

Figure 2. Quantification of publications cited in Pubmed on vitamin D (MESH) in black bars and total publications (all (sb)) in grey, chronologically ordered.

The proposed role of vitamin D in relation to MS and multiple other auto-immune diseases, but also non auto-immune diseases, like cardiovascular diseases, infectious diseases and cancer, has provided the ideal background for vitamin D to become a hype. Some clinicians already prescribe high doses of vitamin D to their patients and some countries included the advice to supplement patients with MS with 2000-4000 IU per day in their protocols. [377] However, hypes can induce false hopes and make it a challenge to disentangle the truth. We presume that the work in this thesis contributes to a better understanding and an increased nuance in the role of vitamin D in MS. Of course we will have to wait for the clinical outcomes of high dose vitamin D supplementation studies first, but findings so far suggest a window of opportunity for vitamin D treatment in pre-clinical and early MS. By extrapolating these results, we can speculate about the relevance of vitamin $D$ supplementation even before the onset of disease. The role of vitamin D later in the disease seems negligible, but long term follow-up of randomised controlled trials have to confirm our findings. One of the mechanisms involved in this might be the maintenance of immunological homeostasis. Immune cells of patients with MS do react differently to vitamin D than those of HC. Our immunological analyses of the blood of patients with MS in a high dose vitamin $D_{3}$ supplementation study showed that vitamin $D$ is possibly able to maintain the immunological balance in the circulating immune cell compartment during the disease course. What the effect of vitamin D is on the immune cells in lymph nodes and in the CNS or on CNS cells themselves, and whether other (environmental) factors in addition to vitamin D play an essential causative role in reported associations, remain open questions. 
Nederlandse samenvatting (Dutch summary) 


\section{Introductie}

In dit proefschrift hebben we een bijdrage willen leveren aan de kennis met betrekking tot de rol van vitamine $D$ in multiple sclerosis (MS). We hebben ons hierbij gericht op de rol van vitamine $D$ spiegels op de ziekteprogressie en beschrijven de effecten van hoge dosis vitamine $D$ suppletie op het immuunsysteem van patiënten met MS.

MS is een inflammatoire ziekte van het centrale zenuwstelstel (CZS), bestaande uit het ruggenmerg en de hersenen. In Nederland hebben ongeveer 16.000 mensen MS, op de wereld ongeveer 1,3 miljoen. Het is een ziekte die zich voornamelijk bij jonge vrouwen manifesteert, tussen de 20 en 40 jaar. De precieze oorzaak is niet bekend; het is hoogstwaarschijnlijk een combinatie van genetische factoren en omgevingsfactoren die een rol speelt. De belangrijkste omgevingsfactoren zijn een lage vitamine $D$ status in het bloed, roken en infecties met bijvoorbeeld het Epstein Barr virus (EBV). Het meest voorkomende type MS is de zogenoemde relapsing remitting MS (RRMS), waarbij periodes van neurologische symptomen (relapsen), afgewisseld worden door periodes van herstel (remissie). De ziekte kan zich met verscheidenheid aan symptomen uiten en deze kunnen bestaan uit gevoelsverlies, tintelingen, vermoeidheid, spierspasmes, zwakte, problemen met lopen, pijn, problemen met het zien, een gestoorde verstandelijke functie, depressie en het niet goed functioneren van blaas en darmen. Als de symptomen niet volledig herstellen en er ook tussen de relapsen verergering van klachten optreedt, spreken we van progressieve MS (secundair progressieve MS (SPMS) of primair progressieve MS (PPMS)).

Er wordt over het algemeen gedacht dat MS een auto-immuunziekte is. Dit geldt in het bijzonder voor het RRMS subtype. Auto-immuunziekten ontstaan door een fout in het immuunsysteem waardoor dit systeem niet goed meer in staat is eigen weefsel van niet-eigen weefsel te onderscheiden. In MS betekent dit dat lichaamseigen weefsel van het CNS door het immuunsysteem wordt aangevallen. Hierdoor raken zenuwcellen (neuronen) en myeline, het vetachtige zenuwmerg dat normaal voor een snelle en vloeiende overdracht van prikkels zorgt, beschadigd. Op de lange termijn zijn deze beschadigingen niet meer te herstellen en treedt verergering van de klachten op.

Vitamine $D$ is een belangrijk vitamine dat wij voornamelijk binnenkrijgen onder invloed van UVB straling in zonlicht. Een goede vitamine D spiegel in het bloed, gemeten als $25(\mathrm{OH}) \mathrm{D}$, is van belang voor een goede calciumregulatie en bothuishouding. Maar lage vitamine D spiegels worden ook in verband gebracht met het risico op het ontwikkelen van autoimmuunziekten als MS. Daarnaast lijkt het erop dat lage vitamine D spiegels in patiënten met MS de kans op het ontwikkelen van relapsen kan vergroten. Een belangrijke open vraag was of vitamine $D$ spiegels ook in verband kunnen worden gebracht met de mate van MS gerelateerde beperkingen en de achteruitgang daarin, later in het ziekteproces. Dit is een belangrijke uitkomstmaat omdat deze achteruitgang van ziekte voornamelijk speelt bij patienten met progressieve MS, voor wie op dit moment alleen behandeling bestaat die symptomen kan verminderen, maar niet de ziekte kan remmen of genezen. 
Voor patiënten met RRMS zijn er wel al verschillende ziekte remmende medicijnen op de markt en vele meer in ontwikkeling. Gebaseerd op de eerdere verbanden die gelegd zijn tussen lage vitamine $D$ spiegels en de kans op het ontwikkelen van een relapse, ontstond de gedachte dat vitamine D suppletie een goede aanvulling zou kunnen zijn op de al bestaande therapieën voor patiënten met RRMS. Eerdere kleine studies hebben uitgewezen dat dit waarschijnlijk veilig is en resultaten waren hoopgevend. Het onderliggende mechanisme van vitamine $D$ is waarschijnlijk een immuunregulatoir effect, waardoor het immuunsysteem minder snel ontspoort.

In dit proefschrift hebben we allereerst de rol van vitamine $D$ in de achteruitgang van de ziekte onderzocht. Daarnaast hebben we in een grootschalige gerandomiseerde en gecontroleerde studie gekeken naar de effecten van vitamine $D$ suppletie op immunologische uitkomstmaten (de SOLARIUM studie).

\section{Samenvatting van het beschreven onderzoek}

Om te beginnen, hebben we in hoofdstuk 1 de in vivo effecten van vitamine D op het immuunsysteem van gezonde controles en van patiënten met een auto-immuunziekte beschreven. Hierin wordt een overzicht gemaakt van de kennis op het moment van de start van het onderzoek dat in dit proefschrift is beschreven. Het mechanisme dat het verband tussen vitamine $D$ en de ziekteactiviteit van patiënten met MS zou kunnen verklaren, is gebaseerd op de gedachte dat vitamine D immuun regulerende eigenschappen heeft. In vitro studies (studies buiten het lichaam van een levend organisme) en studies in het diermodel van MS, experimentele auto-immuun encefalitis ( $E A E$, ontsteking van het encephalon/ brein), hadden eerder aangetoond dat immuun cellen functionele doelwitten zijn van vitamine $D$. In dit overzichtsartikel bleek echter dat de resultaten die tot dan toe bij mensen beschreven waren, heterogeen waren. Bovendien was er sprake van een grote verscheidenheid aan immuun parameters die bestudeerd werd. Over het algemeen leek het erop dat er een trend zichtbaar was richting een anti-inflammatoire rol van vitamine $D$, maar dit kon niet bevestigd worden door overtuigend bewijs. Daarom waren er krachtige studies, zoals gerandomiseerde gecontroleerde onderzoeken nodig, voordat er definitieve conclusies met betrekking tot dit onderwerp getrokken konden worden.

Terwijl dit soort gerandomiseerd en gecontroleerd, grootschalig onderzoek van start ging, hebben wij de rol van fysiologische niveaus vitamine $D$ in het bloed, op progressie van de ziekte onderzocht. Progressie van ziekte is een belangrijke uitkomstmaat, in het bijzonder voor progressieve MS patiënten. Op dit moment zijn er geen medicijnen beschikbaar die ook daadwerkelijk de ziekteprogressie stoppen. Vitamine D zou hierin mogelijk wel een rol kunnen spelen, door een direct positief effect op de cellen van het brein. In hoofdstuk 2 hebben we gebruik gemaakt van de EDSS score om in een retrospectieve follow-up studie van 3 jaar, de relatie tussen vitamine $D$ status in het bloed en progressie van de invaliditeit te onderzoeken. Hoewel we de relatie tussen een lage vitamine D status en een toegenomen 
kans op het ontwikkelen van relapsen in jonge patiënten konden bevestigen, konden we geen statistisch significant verband aantonen tussen de mate van invaliditeit of de progressie van invaliditeit in patiënten met RRMS of patiënten met progressieve MS. Een andere manier om ziekte progressie te onderzoeken is beschreven in hoofdstuk 3. Hier hebben we de rol van vitamine $D$ bekeken in de overgang naar een secundair progressieve MS in patienten met RRMS. We konden bevestigen dat patiënten met SPMS lagere 25(OH)D spiegels hadden dan patiënten met RRMS. Cross-sectioneel gemeten en voor het seizoen gecorrigeerde 25(OH)D spiegels van deze RRMS patiënten voorspelden niet of zij in de daaropvolgende 3 jaar overgingen naar een SPMS. Vitamine D spiegels op het moment van diagnose waren echter wel significant lager in RRMS patiënten met een snelle progressie naar SPMS (mediane RRMS duur van 3.5 (1.0-5.7) jaar) vergeleken met vergelijkbare RRMS patiënten met een langere RRMS duur (minimum mediane RRMS duur 7.7 (6.3-10.0) jaar). Langdurige follow-up van hoge dosis vitamine $D$ suppletie studies zullen de bevindingen in deze beide klinische studies m.b.t. de relatie tussen vitamine $D$ en ziekteprogressie moeten bevestigen. In het tweede gedeelte van dit proefschrift, zijn de effecten van vitamine $D$ op immunologische uitkomstmaten beschreven. De meest recent ontdekte T-helper cel, die in het EAE diermodel, sterk encephalitogeen (dat wil zeggen die een ontsteking in de hersenen kan veroorzaken) is, was de GM-CSF producerende T helper cel. Hoewel er een klinische trial in MS patiënten is geïnitieerd (NCT01517282), zijn data m.b.t. deze cellen in mensen schaars. Daarom, hebben wij deze cellen en de mechanismen die deze cellen reguleren bekeken in hoofdstuk 4. Wij hebben laten zien dat het aantal GM-CSF producerende T cellen in het circulerende immuun cel compartiment niet verschilt tussen patiënten met MS en gezonde controles. Daarnaast zagen we dat GM-CSF producerende cellen 39\% van de T cellen in de hersenvloeistof van zowel patiënten met MS als van controle patiënten uitmaakten. Deze resultaten konden zodoende niet een specifieke pathogene rol van deze cellen in MS bevestigen. In vitro konden regulatoire T cellen uit het bloed en vitamine D de hoeveelheid GMCSF, geproduceerd door T cellen, beïnvloeden. Deze regulatie door vitamine $D$ was minder effectief in MS patiënten.

Het belangrijkste onderdeel van dit proefschrift richt zich op de immunologische effecten van hoge dosis vitamine $D$ suppletie in een gerandomiseerde studie bij patiënten met RRMS. Wij verwachtten dat vitamine $D$ een rol heeft in de immuun regulatie en hierin speelt het cytokine IL-10 een belangrijke rol. Het detecteren van T helper cellen die IL-10 produceren is echter lastig, maar wel belangrijk om de effecten van vitamine $D$ op het immuunsysteem juist in te schatten. Daarom beschrijven we in hoofdstuk 5 een verbeterde methode om deze cellen te detecteren m.b.v. flow cytometrie. Door het weglaten van de eiwit transport remmer monensine uit het protocol, neemt de detectie van het aantal IL-10 producerende $\mathrm{T}$ helper, NK- en NK-T cellen toe, maar niet het aantal B-cellen. In hoofdstuk 6, worden de resultaten van de SOLARIUM studie beschreven. We laten zien dat hoge dosis vitamine $D_{3}$ suppletie geen duidelijke effecten heeft op het aantal, voor MS relevante, pathogene en regulatoire lymfocyten. De anti-inflammatoire IL-4 producerende T helper cellen namen wel af over de follow-up tijd in de placebo groep en dit aantal was stabiel in de vitamine $D_{3}$ 
gesuppleerde groep, terwijl dit niet terug te zien was in een verschil van de IFN- $\gamma / \mathrm{IL}-4$ ratio. Functionele analyse van het supernatant van PBMC na $\mathrm{T}$ cel stimulatie liet een toename van cytokines zien in de placebo groep. Deze was het meest uitgesproken voor de antiinflammatoire cytokines. De cytokine expressie in de vitamine $D_{3}$ gesuppleerde groep veranderde niet. Dit zou kunnen wijzen op een rol van in vivo vitamine $D_{3}$ suppletie in het behoud van homeostase van het adaptieve immuunsysteem vroeg in het beloop van MS. Belangrijk om te vermelden is daarnaast, dat lymfocyten van beide groepen nog steeds reageerden op in vitro $1,25(\mathrm{OH}) \mathrm{D}$ toevoeging. Of het behoud van deze balans ook tot uiting komt in klinische uitkomstmaten zal in de nabije toekomst duidelijk worden.

\section{Vitamine D in MS, hype of hoop?}

De rol van vitamine D in MS zou tweeledig kunnen zijn. Enerzijds is het een van de omgevingsfactoren die geassocieerd is met het risico op het ontwikkelen van MS. Anderzijds, is een lage vitamine $D$ status in het bloed gerelateerd aan de ziekteactiviteit van MS patiënten. Het onderzoek in dit proefschrift heeft met name dit laatste onderwerp verder uitgediept. Het onderzoek naar vitamine $D$ en het aantal artikelen dat hierover gepubliceerd is, is de laatste tientallen jaren enorm toegenomen. Dit feit bevestigt de toegenomen aandacht voor vitamine $D$ in de medisch wetenschappelijke wereld. Daarnaast heeft de mogelijke rol van vitamine $D$ in relatie tot MS en verscheidene andere auto-immuunziekten, maar ook in bijvoorbeeld cardiovasculaire ziekten, infectieuze ziekten en kanker, de ideale voedingsbodem gegeven om uit te groeien tot een hype. Sommige artsen schrijven zelfs al hoge dosis vitamine $D$ voor aan hun patiënten. Ook wordt er in sommige landen al geadviseerd aan patiënten met MS om 2000-4000 internationale eenheden vitamine D per dag te gebruiken. Hypes kunnen er echter voor zorgen dat er valse hoop gecreëerd word en het wordt dan een uitdaging om de waarheid te achterhalen. Wij zijn van mening dat het werk in dit proefschrift bijdraagt aan een beter begrip en een verfijndere blik op de rol van vitamine $D$ in MS. Dit onderzoek zal bijdragen tot het beter onderscheiden wanneer en voor welke patiënt met MS, vitamine D suppletie van nut kan zijn. Natuurlijk moeten we wachten op de klinische uitkomsten van hoge dosis vitamine $D$ suppletie studies, maar het onderzoek tot nu toe wijst op de beste kansen voor vitamine D behandeling in vroege, en misschien wel preklinische, MS. Als we deze resultaten extrapoleren naar preventie, is het de vraag of vitamine D suppletie niet zelfs het meest relevant zou kunnen zijn vóór de start van de ziekte. De rol van vitamine $D$ in de latere fase van de ziekte lijkt verwaarloosbaar, maar langdurige follow-up van gerandomiseerde en gecontroleerde suppletie studies zal dit moeten bevestigen. Eén van de onderliggende mechanismen kan de invloed van vitamine $D$ op het behoud van de immunologische balans zijn. Immuun cellen van patiënten met MS reageren anders op vitamine D dan cellen van gezonde controles. Onze immunologisch analyses van het immuunsysteem van patiënten met MS in een hoge dosis vitamine $D_{3}$ suppletie studie lieten zien dat vitamine D mogelijk in staat is de immunologische balans van het immuunsysteem, vroeg in de ziekte, te bewaren. Wat er echter gebeurt met immuun cellen in lymfe klieren en in het brein, of met 
de cellen van het brein zelf, en of ook andere (omgevings) factoren naast vitamine $D$ een essentiële rol spelen in de beschreven verbanden, blijven open vragen. 
Valorisation 


\section{Introduction}

In the final chapter of this thesis, we will elaborate on how to make the knowledge, obtained in our scientific research and reported in this thesis, work. What can our results add to society, to whom, with whom and how? From a scientific point of view, there are still a lot of open questions in the field of multiple sclerosis and auto-immunity. The MS society in the UK tried to identify a top 10 of important research questions that are most crucial to be solved according to people having MS and their health professionals. [378]

- Which treatments are effective to slow, stop or reverse the accumulation of disability associated with MS?

- How can MS be prevented?

- Which treatments are effective for fatigue in people with MS?

- How can people with MS be best supported to self-manage their condition?

- Does early treatment with aggressive disease modifying drugs improve the prognosis for people with MS?

- Is vitamin D supplementation an effective disease modifying treatment for MS?

- Which treatments are effective to improve mobility for people with MS?

- Which treatments are effective to improve cognition in people with MS?

- Which treatments are effective for pain in people with MS?

- Is physiotherapy effective in reducing disability in people with MS?

Besides the effectiveness of vitamin D supplementation, at least three others questions among these 10 questions were touched upon in this thesis. Behind these questions lay a couple of even more basic questions about the disease mechanism in MS. How would it be possible to prevent MS, or to treat disability, fatigue and cognitive problems, if we still don't know what exactly happens in the pathophysiology, if we do not know what we measure and if we do not know how to measure it? In the question about the role of vitamin $D$ we have made some important steps forward in the last years, investigating the disease mechanism and the clinical outcomes. Now, it is the question how we can continue to valorise these results in a process of value creation and making scientific knowledge available by translation, application, interaction and inspiration. [379, 380] 


\section{Translating from bench-to bedside: vitamin D in multiple sclerosis}

\section{Purpose of studying the role of vitamin D in MS}

As has been described in the general introduction of this thesis, vitamin D deficiency has been associated with different diseases. [1] One of the diseases associated with a vitamin D deficiency, especially during childhood and adolescence, is MS. [70] Two main important scientific challenges in MS are to unravel the pathological mechanism and to develop new effective therapies with a minimum of (serious) side effects. While vitamin D deficiency has been identified as a risk factor for developing MS and has been proposed as a(n add-on) therapy decreasing disease activity, studying vitamin D in MS is very interesting from a scientific point of view.

Furthermore, there are other important reasons to study the role of vitamin $D$ in multiple sclerosis. MS is a disabling disease mainly affecting young women, and to a lesser extent men, in the prime of their lives, many of them raising kids. Therefore, this disease will not only affect the physical and economic independence of those people but also of the people surrounding them, and of society. In the Netherlands, the total health costs for MS were 244 million euro in 2011 ( $0.3 \%$ of total costs spent in the healthcare). [381] Patients with MS experience physical disability with the need for a walking stick or wheelchair on average 20 years and 30 years after diagnosis, respectively. [382] Already at the time of a first clinical attack, $50 \%$ of the patients have cognitive impairment in at least two cognitive areas. [383] Together this involves a significant emotional and social burden: more than $50 \%$ of patients with MS have to decrease the amount of hours that they work or have to change to other functions [384] and they have higher probabilities to separate or get divorced compared to the general population [385]. Until now, there is no cure available and treatment focuses on the prevention of relapses and disability progression. Vitamin D could possibly be an interesting and relative cheap target for prevention and treatment of MS. However, last years, vitamin D has become a hype, and has sometimes been presented as the new panacea. With that it has become increasingly difficult to get to know the truth about the efficiency of vitamin D supplementation. Patients and their treating physicians want to know whether or not they should prescribe or take (high dose) vitamin D supplementation and when and in which dose. Policy makers need to know what choices they should make in preventive medicine and also funding bodies, who have to divide research budgets among a number of projects, need to be able to identify the most important research. All these decisions should be based on facts, not on hypes. We aimed to increase our knowledge about the role of vitamin $\mathrm{D}$ in multiple sclerosis, by collecting scientific data of well-considered research. 


\section{Main results described in this thesis which need further valorisation}

In this thesis, the effects of vitamin $D$ in multiple sclerosis have been investigated from both a clinical and immunological perspective. With that, the research in this thesis is translational research (from bench to bedside) [386] and in part already entered the process of valorisation.

- Taking immunological outcomes as a read-out, we saw that data on the in vivo effects of vitamin $D$ in health and disease were heterogeneous in many aspects. A trend towards an anti-inflammatory role of vitamin $\mathrm{D}$ seemed to be present, but it was difficult to draw firm conclusions (reviewed in chapter $\mathbf{1}$ )

- From a clinical point of view an important gap in our knowledge was the role of vitamin $D$ in disease progression. Disease progression is one of the most important hallmarks of disability and has major impacts on the quality of life. We could demonstrate that vitamin $D$ levels are predictors of disease activity and probably also the time to conversion to progressive disease, but only at the start of the disease and not later on, during the disease course (chapter $\mathbf{2}$ and $\mathbf{3}$ ).

- From the bench side view, we improved the technique to discover IL-10, one of the important cytokines in our body, in terms of immune regulation (chapter 4).

- $\quad$ From the bench to bedside view, we looked at the role of GM-CSF in human MS: GM$\mathrm{CSF}^{+} \mathrm{T}$ cells were equally present in patients and controls, and were increased in CSF compared to blood. We discovered that the number of $\mathrm{GM}^{-\mathrm{CSF}^{+}} \mathrm{T}$ cells are probably not affected by vitamin $D$, but functionally they were regulated by regulatory $T$ cells and vitamin $D$, the latter one being less effective in patients with MS than in healthy controls (chapter 5).

- In a RCT, already on our way to valorisation of vitamin D supplementation, we showed that high dose vitamin $\mathrm{D}$ supplementation is not able to promote immune cell balance, but that it is able to prevent further disturbance of the immune balance during the disease course in early MS (chapter 6)

\section{The innovative character of our studies}

With the results from our studies we can make some steps further in finding an answer to the "hype or hope" question. From our clinical studies we can make more clear what the target group of patients is, which will benefit most from (high dose) vitamin D supplementation. These are the patients at the start of their disease or patients or even healthy individuals in the pre-clinical phase of the disease. With our immunological studies on GM-CSF and vitamin $D$, we have aimed to translate findings from animal and in vitro research to in vivo effects in humans. The added value of the SOLARIUM study compared to previous studies was that this was a placebo controlled study with relevant power, and a high dose $(14.000$ IU/day) and long term (48 weeks) vitamin $D_{3}$ supplementation intervention. Furthermore, another point of interest is that we looked at the regulatory immune cell compartment and $T$ cell compartment as a whole in this study, not focusing on a single cell or cytokine. [387] 


\section{Application, interaction and inspiration}

\section{Applicable science}

The studies described in this thesis are able to contribute to the use of scientific knowledge in different ways.

First of all, they increase our understanding in the pathophysiology and the role of environmental factors in the disease multiple sclerosis and in auto-immunity. Furthermore, with our research on GM-CSF producing T cells, we added new information to the field, information that should be kept in mind when developing GM-CSF therapies in MS. [388]

Second, with regard to the vitamin D supplementation therapy, we showed that it can overcome a further imbalance in the immune system during the course of the disease. Of course, clinical outcomes have to confirm our findings, but if so, we have hints towards a narrow window of opportunity for vitamin $D$ supplementation at the start of the disease and should further investigate the optimal dose. These recommendations should then be incorporated in guidelines for general practitioners and neurologists treating patients with auto-immune diseases like MS. With regard to high dose vitamin D supplementation two other options might be worthwhile to deliberate on. First, it might be worthwhile to investigate the effectiveness of high dose vitamin D supplementation in the pre-clinical phase, during childhood and adolescence in people sensitive to developing auto-immune diseases. This probability to develop an auto-immune disease should possibly take into account an individuals' genetic make-up, month of birth, place of birth, family history and ethnicity. Second, as already deliberated on in the general discussion of this thesis, it might be lucrative to think about in vitro treatment of autologous immune cells, for example T cells, with vitamin D and replacement of those cells in the human body. Hereby, the in vivo (compensatory) effects, as shown in our vitamin D supplementation study, might be circumvented and efficacy increased. [347]

\section{Implementation by interaction and inspiration}

Implementation of scientific knowledge is not only about "knowledge, skills and cash". It also includes more "soft skills" for example communication, cooperation and collaboration. [379] Our research is of course first of all most interesting for (future) patients with MS and their treating physicians. They should be informed about our research activities. Efforts have already been made via the website of the Academic MS Centre, via patient associations and via presentations at patient days. Our studies were published in (bio)medical journals and presented at national and international congresses to reach treating physicians and colleagues from different biomedical disciplines, like the neurology, immunology and vitamin $D$ field. Future colleagues had the opportunity to learn about our research during lectures for students in the medical and biomedical field and for nurses, or by performing an internship in our group. The "7 UMC tour", a day organised to celebrate the 50 years birthday of the Dutch Society of Immunology in 2014, was an example of an opportunity we took to also inform the general public about our research. 
If our immunological results in the SOLARIUM study will be confirmed by positive results of clinical outcomes in the SOLAR trial, it will become important to reach consensus if, when and how to prescribe high dose vitamin D supplementation to patients with MS and to incorporate these advices in national and international guidelines. To fasten the process of a possible implementation of GM-CSF therapy and vitamin D supplementation therapy, also the expertise of pharmaceutical companies and policy makers should be used for optimal valorisation. Pharmaceutical companies will have added value by their knowledge on implementation of drugs to the market and, especially if there are more clues pointing to preventive medicine, policy makers should get involved to make people aware of the sense and nonsense about vitamin D. Lastly, if in vitro treatment of T cells with vitamin D is promising enough to enter a clinical phase study, clinical immunologists should stay involved.

Apart from these hopes for the future there are also still some challenges to tackle: MS is a very heterogeneous disease, and our results are not that easy translatable to all MS patients. Furthermore, big challenges are there for (bio)medical research in general: how to deal with big datasets, how to increase reproducibility, how to objectively analyse data, how to make translation from animal to human more efficient [353, 354, 373, 374], how to build bridges between the different research areas [371, 372] and different professional groups in healthcare, how to make it possible that negative data get the same attention as positive data [375] and how to reduce waste and maximise efficiency of research sources? Some initiatives have already started (for example the REWARD (REduce research Waste And Reward Diligence) Campaign of the Lancet and the Dutch "Science in Transition" initiative that aims to introduce new checks and balances in the scientific system and inform layman about the decisions made [396, 397]), but we all have to think and work together on that. Only then valorisation of research as that described in this thesis will become easier and more efficient in the future.

\section{Conclusion}

Multiple sclerosis is a disabling disease, with much impact on individuals, but also on society. This thesis touches upon several of the most important research questions in this disease with a special attention to the role of vitamin D. Approached from a clinical and immunological perspective, this thesis already describes translational research. Further valorisation needs to be done by staying critical towards research methods and results of ourselves and of others and continuing the communication and collaboration with different disciplines, with some important challenges for the biomedical field ahead for the future. 


\section{References}


1. Grant W.B., Diseases affected by vitamin D: sun exposure, in Handbook of vitamin D in human health, R. Watson, Editor. 2013, Wageningen Academic Publishers. p. 206-224.

2. Smolders J., Vitamin $d$ and multiple sclerosis: correlation, causality, and controversy. Autoimmune Dis, 2011. 2011: p. 629538.

3. Jongen L. Lidwina of Schiedam. Online Dictionary of Dutch Women 2014; Available from:

http://resources.huygens.knaw.nl/vrouwenle xicon/lemmata/en.

4. Medaer R., Does the history of multiple sclerosis go back as far as the 14th century? Acta Neurol Scand, 1979. 60(3): p. 189-192.

5. Compston A., The story of multiple sclerosis, in Mc Alpine's Multiple Sclerosis. 1998, Churchill Livingstone: London. p. 3-42

6. Ebringer A., History of the Attempts to Find the Origin of Multiple Sclerosis, in Multiple Sclerosis, Mad Cow Disease and Acinetobacter. 2015, Springer International Publishing: Switserland. p. 9-14.

7. Olitsky P.K. and Yager R.H., Experimental disseminated encephalomyelitis in white mice. J Exp Med, 1949. 90(3): p. 213-224.

8. Rivers T.M. and Schwentker F.F., Encephalomyelitis Accompanied by Myelin Destruction Experimentally Produced in Monkeys. J Exp Med, 1935. 61(5): p. 689-702.

9. Rivers T.M., Sprunt D.H., and Berry G.P., Observations on Attempts to Produce Acute Disseminated Encephalomyelitis in Monkeys. J Exp Med, 1933. 58(1): p. 39-53.

10. World Health Organization, Atlas multiple sclerosis resources in the world 2008. WHO Press: Geneva, Switserland.

11. Gommer A.M. and Poos M.J.J.C. Achtergrondcijfers bij rangordetabellen VTV-2010. Volksgezondheid Toekomst Verkenning, Nationaal Kompas Volksgezondheid. 2010 Available from: http://www.nationaalkompas.nl> Nationaal Kompas Volksgezondheid \Gezondheidstoestand \Ziekten en aandoeningen.

12. Hoffjan S. and Akkad D.A., The genetics of multiple sclerosis: an update 2010. Mol Cell Probes, 2010. 24(5): p. 237-243.
13. Olerup O. and Hillert J., HLA class IIassociated genetic susceptibility in multiple sclerosis: a critical evaluation. Tissue Antigens, 1991. 38(1): p. 1-15.

14. International Multiple Sclerosis Genetics Consortium, Wellcome Trust Case Control Consortium, Sawcer S., Hellenthal G., Pirinen M., Spencer C.C., et al., Genetic risk and a primary role for cell-mediated immune mechanisms in multiple sclerosis. Nature, 2011. 476(7359): p. 214-219.

15. Fugger L., Friese M.A., and Bell J.I., From genes to function: the next challenge to understanding multiple sclerosis. Nat Rev Immunol, 2009. 9(6): p. 408-417.

16. Sawcer S., Franklin R.J., and Ban M., Multiple sclerosis genetics. Lancet Neurol, 2014. 13(7): p. 700-709.

17. Lossius A., Johansen J.N., Torkildsen O., Vartdal F., and Holmoy T., Epstein-Barr virus in systemic lupus erythematosus, rheumatoid arthritis and multiple sclerosisassociation and causation. Viruses, 2012. 4(12): p. 3701-3730.

18. Owens G.P. and Bennett J.L., Trigger, pathogen, or bystander: the complex nexus linking Epstein- Barr virus and multiple sclerosis. Mult Scler, 2012. 18(9): p. 1204-1208.

19. Compston A. and Coles A., Multiple sclerosis. Lancet, 2008. 372(9648): p. 1502-1517.

20. Schumacher G.A., Beebe G., Kibler R.F., Kurland L.T., Kurtzke J.F., McDowell F., et al., Problems of Experimental Trials of Therapy in Multiple Sclerosis: Report by the Panel on the Evaluation of Experimental Trials of Therapy in Multiple Sclerosis. Ann N Y Acad Sci, 1965. 122: p. 552-568.

21. Poser C.M., Paty D.W., Scheinberg L., McDonald W.I., Davis F.A., Ebers G.C., et al., New diagnostic criteria for multiple sclerosis: guidelines for research protocols. Ann Neurol, 1983. 13(3): p. 227-231.

22. McDonald W.I., Compston A., Edan G., Goodkin D., Hartung H.P., Lublin F.D., et al., Recommended diagnostic criteria for multiple sclerosis: guidelines from the International Panel on the diagnosis of multiple sclerosis. Ann Neurol, 2001. 50(1): p. 121127. 
23. Polman C.H., Reingold S.C., Banwell B., Clanet M., Cohen J.A., Filippi M., et al., Diagnostic criteria for multiple sclerosis: 2010 revisions to the McDonald criteria. Ann Neurol, 2011. 69(2): p. 292-302.

24. Polman C.H., Reingold S.C., Edan G., Filippi M., Hartung H.P., Kappos L., et al., Diagnostic criteria for multiple sclerosis: 2005 revisions to the "McDonald Criteria". Ann Neurol, 2005. 58(6): p. 840-846.

25. Miller D., Barkhof F., Montalban X., Thompson A., and Filippi M., Clinically isolated syndromes suggestive of multiple sclerosis, part I: natural history, pathogenesis, diagnosis, and prognosis. Lancet Neurol, 2005. 4(5): p. 281-288.

26. Okuda D.T., Mowry E.M., Beheshtian A., Waubant E., Baranzini S.E., Goodin D.S., et al., Incidental MRI anomalies suggestive of multiple sclerosis: the radiologically isolated syndrome. Neurology, 2009. 72(9): p. 800805.

27. Stinissen $P$. and Hellings N., Activation of myelin reactive $T$ cells in multiple sclerosis: $a$ possible role for T cell degeneracy? Eur J Immunol, 2008. 38(5): p. 1190-1193.

28. Lunemann J.D., Jelcic I., Roberts S., Lutterotti A., Tackenberg B., Martin R., et al., EBNA1specific $T$ cells from patients with multiple sclerosis cross react with myelin antigens and co-produce IFN-gamma and IL-2. J Exp Med, 2008. 205(8): p. 1763-1773.

29. Lang H.L., Jacobsen H., Ikemizu S., Andersson C., Harlos K., Madsen L., et al., A functional and structural basis for TCR cross-reactivity in multiple sclerosis. Nat Immunol, 2002. 3(10): p. 940-943.

30. van Noort J.M., van Sechel A.C., Bajramovic J.J., el Ouagmiri M., Polman C.H., Lassmann $H_{\text {., }}$ et al., The small heat-shock protein alpha B-crystallin as candidate autoantigen in multiple sclerosis. Nature, 1995. 375(6534): p. 798-801.

31. Srivastava R., Aslam M., Kalluri S.R., Schirmer L., Buck D., Tackenberg B., et al., Potassium channel KIR4.1 as an immune target in multiple sclerosis. N Engl J Med, 2012. 367(2): p. 115-123.

32. de Bock L., Somers K., Fraussen J., Hendriks J.J., van Horssen J., Rouwette M., et al.,
Sperm-associated antigen 16 is a novel target of the humoral autoimmune response in multiple sclerosis. J Immunol, 2014. 193(5): p. 2147-2156.

33. Reindl M., Khalil M., and Berger T., Antibodies as biological markers for pathophysiological processes in MS. J Neuroimmunol, 2006. 180(1-2): p. 50-62.

34. Amedei A., Prisco D., and D'Elios M.M., Multiple sclerosis: the role of cytokines in pathogenesis and in therapies. Int J Mol Sci, 2012. 13(10): p. 13438-13460.

35. Opdenakker G. and Van Damme J., Cytokineregulated proteases in autoimmune diseases. Immunol Today, 1994. 15(3): p. 103-107.

36. Gutcher I. and Becher B., APC-derived cytokines and $\mathrm{T}$ cell polarization in autoimmune inflammation. J Clin Invest, 2007. 117(5): p. 1119-1127.

37. Park H., Li Z., Yang X.O., Chang S.H., Nurieva R., Wang Y.H., et al., A distinct lineage of CD4 $T$ cells regulates tissue inflammation by producing interleukin 17. Nat Immunol, 2005. 6(11): p. 1133-1141.

38. Codarri L., Gyulveszi G., Tosevski V., Hesske L., Fontana A., Magnenat L., et al., RORgammat drives production of the cytokine GMCSF in helper T cells, which is essential for the effector phase of autoimmune neuroinflammation. Nat Immunol, 2011. 12(6): p. 560-567.

39. Noster R., Riedel R., Mashreghi M.F., Radbruch H., Harms L., Haftmann C., et al., IL-17 and GM-CSF expression are antagonistically regulated by human $T$ helper cells. Sci Transl Med, 2014. 6(241): p. 241ra280.

40. Rose N.R. and Bona C., Defining criteria for autoimmune diseases (Witebsky's postulates revisited). Immunol Today, 1993. 14(9): p. 426-430.

41. Damoiseaux J.G. and Cohen Tervaert J.W., The definition of autoimmune disease: are Koch's postulates applicable? Neth J Med, 2002. 60(7): p. 266-268.

42. Simmons S.B., Pierson E.R., Lee S.Y., and Goverman J.M., Modeling the heterogeneity of multiple sclerosis in animals. Trends Immunol, 2013. 34(8): p. 410-422.

43. Stys P.K., Zamponi G.W., van Minnen J., and Geurts J.J., Will the real multiple sclerosis 
please stand up? Nat Rev Neurosci, 2012. 13(7): p. 507-514.

44. D'Haeseleer M., Cambron M., Vanopdenbosch L., and De Keyser J., Vascular aspects of multiple sclerosis. Lancet Neurol, 2011. 10(7): p. 657-666.

45. D'Haeseleer M., Hostenbach S., Peeters I., Sankari S.E., Nagels G., De Keyser J., et al., Cerebral hypoperfusion: a new pathophysiologic concept in multiple sclerosis? J Cereb Blood Flow Metab, 2015. 35(9): p. 14061410.

46. Filippi M. and Rocca M.A., The multiple sclerosis mystery: is there a vascular component? Lancet Neurol, 2011. 10(7): p. 597-598.

47. Galea I., Bechmann I., and Perry V.H., What is immune privilege (not)? Trends Immunol, 2007. 28(1): p. 12-18.

48. Ransohoff R.M. and Engelhardt B., The anatomical and cellular basis of immune surveillance in the central nervous system. Nat Rev Immunol, 2012. 12(9): p. 623-635.

49. Aspelund A., Antila S., Proulx S.T., Karlsen T.V., Karaman S., Detmar M., et al., A dural lymphatic vascular system that drains brain interstitial fluid and macromolecules. J Exp Med, 2015. 212(7): p. 991-999.

50. Louveau A., Smirnov I., Keyes T.J., Eccles J.D., Rouhani S.J., Peske J.D., et al., Structural and functional features of central nervous system lymphatic vessels. Nature, 2015. 523(7560): p. 337-341.

51. Goodin D.S., Frohman E.M., Garmany G.P. Jr., Halper J., Likosky W.H., Lublin F.D., et al., Disease modifying therapies in multiple sclerosis: report of the Therapeutics and Technology Assessment Subcommittee of the American Academy of Neurology and the MS Council for Clinical Practice Guidelines. Neurology, 2002. 58(2): p. 169-178.

52. Palmer A.M., Pharmacotherapeuetic options for the treatment of multiple sclerosis. Clin Med Insights, 2012. 4: p. 1-24.

53. Miller D.H., Weber T., Grove R., Wardell C., Horrigan J., Graff O., et al., Firategrast for relapsing remitting multiple sclerosis: a phase 2, randomised, double-blind, placebocontrolled trial. Lancet Neurol, 2012. 11(2): p. 131-139.
54. Liu J., Wang L.N., Zhan S., and Xia Y., Daclizumab for relapsing remitting multiple sclerosis. Cochrane Database Syst Rev, 2013. 12: p. CD008127.

55. Varrin-Doyer M., Zamvil S.S., and SchulzeTopphoff U., Laquinimod, an up-and-coming immunomodulatory agent for treatment of multiple sclerosis. Exp Neurol, 2014. $262 \mathrm{Pt}$ A: p. 66-71.

56. Barun B. and Bar-Or A., Treatment of multiple sclerosis with anti-CD20 antibodies. Clin Immunol, 2012. 142(1): p. 31-37.

57. Radaelli M., Merlini A., Greco R., Sangalli F., Comi G., Ciceri F., et al., Autologous bone marrow transplantation for the treatment of multiple sclerosis. Curr Neurol Neurosci Rep, 2014. 14(9): p. 478.

58. Tran J.Q., Rana J., Barkhof F., Melamed I., Gevorkyan H., Wattjes M.P., et al., Randomized phase I trials of the safety/tolerability of anti-LINGO-1 monoclonal antibody BIIB033. Neurol Neuroimmunol Neuroinflamm, 2014. 1(2): p. e18.

59. Pirko I., Ciric B., Gamez J., Bieber A.J., Warrington A.E., Johnson A.J., et al., A human antibody that promotes remyelination enters the CNS and decreases lesion load as detected by T2-weighted spinal cord MRI in a virus-induced murine model of MS. FASEB J, 2004. 18(13): p. 1577-1579.

60. Bomprezzi R., Dimethyl fumarate in the treatment of relapsing-remitting multiple sclerosis: an overview. Ther Adv Neurol Disord, 2015. 8(1): p. 20-30.

61. Bar-Or A., Pachner A., Menguy-Vacheron F., Kaplan J., and Wiendl H., Teriflunomide and its mechanism of action in multiple sclerosis. Drugs, 2014. 74(6): p. 659-674.

62. Sanford M., Fingolimod: a review of its use in relapsing-remitting multiple sclerosis. Drugs, 2014. 74(12): p. 1411-1433.

63. Ruck T., Bittner S., Wiendl H., and Meuth S.G., Alemtuzumab in Multiple Sclerosis: Mechanism of Action and Beyond. Int J Mol Sci, 2015. 16(7): p. 16414-16439.

64. Xu Z., Zhang F., Sun F., Gu K., Dong S., and He D., Dimethyl fumarate for multiple sclerosis. Cochrane Database Syst Rev, 2015. 4: p. CD011076. 
65. Arnon R. and Aharoni R., Mechanism of action of glatiramer acetate in multiple sclerosis and its potential for the development of new applications. Proc Natl Acad Sci U S A, 2004. 101 Suppl 2: p. 14593-14598.

66. Yong V.W., Chabot S., Stuve O., and Williams G., Interferon beta in the treatment of multiple sclerosis: mechanisms of action. Neurology, 1998. 51(3): p. 682-689.

67. Rudick R.A. and Sandrock A., Natalizumab: alpha 4-integrin antagonist selective adhesion molecule inhibitors for MS. Expert Rev Neurother, 2004. 4(4): p. 571-580.

68. Simpson S., Jr., Blizzard L., Otahal P., Van der Mei I., and Taylor B., Latitude is significantly associated with the prevalence of multiple sclerosis: a meta-analysis. J Neurol Neurosurg Psychiatry, 2011. 82(10): p. 1132-1141.

69. Verheul F., Smolders J., Trojano M., Lepore V., Zwanikken C., Amato M.P., et al., Fluctuations of MS births and UV-light exposure. Acta Neurol Scand, 2013. 127(5): p. 301-308.

70. Ascherio A., Munger K.L., and Simon K.C., Vitamin $D$ and multiple sclerosis. Lancet Neurol, 2010. 9(6): p. 599-612.

71. Mowry E.M., Krupp L.B., Milazzo M., Chabas D., Strober J.B., Belman A.L., et al., Vitamin D status is associated with relapse rate in pediatric-onset multiple sclerosis. Ann Neurol, 2010. 67(5): p. 618-624.

72. Runia T.F., Hop W.C., de Rijke Y.B., Buljevac D., and Hintzen R.Q., Lower serum vitamin D levels are associated with a higher relapse risk in multiple sclerosis. Neurology, 2012. 79(3): p. 261-266.

73. van der Mei I.A., Ponsonby A.L., Dwyer T., Blizzard L., Taylor B.V., Kilpatrick T., et al., Vitamin D levels in people with multiple sclerosis and community controls in Tasmania, Australia. J Neurol, 2007. 254(5): p. 581-590.

74. Simpson S. Jr., Taylor B., Blizzard L., Ponsonby A.L., Pittas F., Tremlett $H_{\text {., }}$ et al., Higher 25-hydroxyvitamin $D$ is associated with lower relapse risk in multiple sclerosis. Ann Neurol, 2010. 68(2): p. 193-203.

75. Burton J.M., Kimball S., Vieth R., Bar-Or A., Dosch H.M., Cheung R., et al., A phase I/II dose-escalation trial of vitamin D3 and calcium in multiple sclerosis. Neurology, 2010. 74(23): p. 1852-1859.
76. James E., Dobson R., Kuhle J., Baker D., Giovannoni G., and Ramagopalan S.V., The effect of vitamin D-related interventions on multiple sclerosis relapses: a meta-analysis. Mult Scler, 2013. 19(12): p. 1571-1579.

77. Kampman M.T., Steffensen L.H., Mellgren S.I., and Jorgensen L., Effect of vitamin D3 supplementation on relapses, disease progression, and measures of function in persons with multiple sclerosis: exploratory outcomes from a double-blind randomised controlled trial. Multiple sclerosis, 2012. 18(8): p. 11441151.

78. Kimball S.M., Ursell M.R., O'Connor P., and Vieth R., Safety of vitamin D3 in adults with multiple sclerosis. Am J Clin Nutr, 2007. 86(3): p. 645-651.

79. Smolders J., Peelen E., Thewissen M., Cohen Tervaert J.W., Menheere P., Hupperts R., et al., Safety and $T$ cell modulating effects of high dose vitamin D3 supplementation in multiple sclerosis. PLoS One, 2010. 5(12): p. e15235.

80. Soilu-Hanninen M., Aivo J., Lindstrom B.M., Elovaara I., Sumelahti M.L., Farkkila M., et al., A randomised, double blind, placebo controlled trial with vitamin D3 as an add on treatment to interferon beta-1b in patients with multiple sclerosis. J Neurol Neurosurg Psychiatry, 2012. 83(5): p. 565-571.

81. Stein M.S., Liu Y., Gray O.M., Baker J.E., Kolbe S.C., Ditchfield M.R., et al., A randomized trial of high-dose vitamin D2 in relapsingremitting multiple sclerosis. Neurology, 2011. 77(17): p. 1611-1618.

82. Smolders J., Schuurman K.G., van Strien M.E., Melief J., Hendrickx D., Hol E.M., et al., Expression of vitamin $\mathrm{D}$ receptor and metabolizing enzymes in multiple sclerosis-affected brain tissue. J Neuropathol Exp Neurol, 2013. 72(2): p. 91-105.

83. Garcion E., Wion-Barbot N., Montero-Menei C.N., Berger F., and Wion D., New clues about vitamin $D$ functions in the nervous system. Trends Endocrinol Metab, 2002. 13(3): p. 100-105.

84. Smolders J., Moen S.M., Damoiseaux J., Huitinga I., and Holmoy T., Vitamin D in the healthy and inflamed central nervous sys- 
tem: access and function. J Neurol Sci, 2011. 311(1-2): p. 37-43.

85. Smolders J., Hupperts R., and Damoiseaux J., Shining light on Vitamin D and multiple sclerosis, in Translational Neuroimmunology of Multiple Sclerosis: From Disease Mechanisms to Clinical Applications, A. R, Editor. 2016, Elsevier: London (UK). p. 369-387.

86. Roxburgh R.H., Seaman S.R., Masterman T., Hensiek A.E., Sawcer S.J., Vukusic S., et al., Multiple Sclerosis Severity Score: using disability and disease duration to rate disease severity. Neurology, 2005. 64(7): p. 11441151.

87. Liu C., Li Wan Po A., and Blumhardt L.D., "Summary measure" statistic for assessing the outcome of treatment trials in relapsingremitting multiple sclerosis. J Neurol Neurosurg Psychiatry, 1998. 64(6): p. 726-729.

88. Simonian N.A., McAllister A., and Lull J., "Summary measure" statistic for assessing the outcome of treatment trials in relapsingremitting multiple sclerosis. J Neurol Neurosurg Psychiatry, 1999. 66(4): p. 553-554.

89. Sipe J.C., Knobler R.L., Braheny S.L., Rice G.P., Panitch H.S., and Oldstone M.B., A neurologic rating scale (NRS) for use in multiple sclerosis. Neurology, 1984. 34(10): p. 13681372.

90. Hauser S.L., Dawson D.M., Lehrich J.R., Beal M.F., Kevy S.V., Propper R.D., et al., Intensive immunosuppression in progressive multiple sclerosis. A randomized, three-arm study of high-dose intravenous cyclophosphamide, plasma exchange, and ACTH. N Engl J Med, 1983. 308(4): p. 173-180.

91. Cutter G.R., Baier M.L., Rudick R.A., Cookfair D.L., Fischer J.S., Petkau J., et al., Development of a multiple sclerosis functional composite as a clinical trial outcome measure. Brain, 1999. 122 ( Pt 5): p. 871-882.

92. Fisher E., Rudick R.A., Cutter G., Baier M., Miller D., Weinstock-Guttman B., et al., Relationship between brain atrophy and disability: an 8-year follow-up study of multiple sclerosis patients. Mult Scler, 2000. 6(6): p. 373-377.

93. Fisniku L.K., Chard D.T., Jackson J.S., Anderson V.M., Altmann D.R., Miszkiel K.A., et al., Gray matter atrophy is related to long-term disability in multiple sclerosis. Ann Neurol, 2008. 64(3): p. 247-254.

94. Jacobsen C., Hagemeier J., Myhr K.M., Nyland H., Lode K., Bergsland N., et al., Brain atrophy and disability progression in multiple sclerosis patients: a 10-year follow-up study. J Neurol Neurosurg Psychiatry, 2014. 85(10): p. 1109-1115.

95. Miller D.H., Barkhof F., Frank J.A., Parker G.J., and Thompson A.J., Measurement of atrophy in multiple sclerosis: pathological basis, methodological aspects and clinical relevance. Brain, 2002. 125(Pt 8): p. 1676-1695.

96. Fuhr P. and Kappos L., Evoked potentials for evaluation of multiple sclerosis. Clin Neurophysiol, 2001. 112(12): p. 2185-2189.

97. Walsh J.C., Garrick R., Cameron J., and McLeod J.G., Evoked potential changes in clinically definite multiple sclerosis: a two year follow up study. J Neurol Neurosurg Psychiatry, 1982. 45(6): p. 494-500.

98. Comabella M. and Montalban X., Body fluid biomarkers in multiple sclerosis. Lancet $\mathrm{Neu}$ rol, 2014. 13(1): p. 113-126.

99. Teunissen C.E., Malekzadeh A., Leurs C., Bridel C., and Killestein J., Body fluid biomarkers for multiple sclerosis-the long road to clinical application. Nat Rev Neurol, 2015. 11(10): p. 585-596.

100. Abbas A.K., Benoist C., Bluestone J.A., Campbell D.J., Ghosh S., Hori S., et al., Regulatory T cells: recommendations to simplify the nomenclature. Nat Immunol, 2013. 14(4): p. 307-308.

101. Goldberg P., Fleming M.C., and Picard E.H., Multiple sclerosis: decreased relapse rate through dietary supplementation with calcium, magnesium and vitamin D. Med Hypotheses, 1986. 21(2): p. 193-200.

102. Kurtzke J.F., On the fine structure of the distribution of multiple sclerosis. Acta Neurol Scand, 1967. 43(3): p. 257-282.

103. Smolders J., Menheere P., Kessels A., Damoiseaux J., and Hupperts R., Association of vitamin $D$ metabolite levels with relapse rate and disability in multiple sclerosis. Mult Scler, 2008. 14(9): p. 1220-1224.

104. Hayes C.E., Cantorna M.T., and DeLuca H.F., Vitamin $D$ and multiple sclerosis. Proc Soc Exp Biol Med, 1997. 216(1): p. 21-27. 
105. Smolders J. and Damoiseaux J., Vitamin D as a T-cell modulator in multiple sclerosis. Vitam Horm, 2011. 86: p. 401-428.

106. Milo R. and Kahana E., Multiple sclerosis: geoepidemiology, genetics and the environment. Autoimmun Rev, 2010. 9(5): p. A387-394.

107. Rosati G., The prevalence of multiple sclerosis in the world: an update. Neurol Sci, 2001. 22(2): p. 117-139.

108. Compston A. and Coles A., Multiple sclerosis. Lancet, 2002. 359(9313): p. 1221-1231.

109. Bar-Or A., The immunology of multiple sclerosis. Semin Neurol, 2008. 28(1): p. 2945.

110. Sawcer S., Hellenthal G., Pirinen M., Spencer C.C., Patsopoulos N.A., Moutsianas L., et al., Genetic risk and a primary role for cellmediated immune mechanisms in multiple sclerosis. Nature, 2011. 476(7359): p. 214219.

111. Durelli L., Conti L., Clerico M., Boselli D., Contessa G., Ripellino P., et al., T-helper 17 cells expand in multiple sclerosis and are inhibited by interferon-beta. Ann Neurol, 2009. 65(5): p. 499-509.

112. Edwards L.J., Robins R.A., and Constantinescu C.S., Th17/Th1 phenotype in demyelinating disease. Cytokine, 2010. 50(1): p. 19-23.

113. Frisullo G., Nociti V., Iorio R., Patanella A.K., Marti A., Caggiula M., et al., IL17 and IFNgamma production by peripheral blood mononuclear cells from clinically isolated syndrome to secondary progressive multiple sclerosis. Cytokine, 2008. 44(1): p. 22-25.

114. Matusevicius D., Kivisakk P., He B., Kostulas N., Ozenci V., Fredrikson S., et al., Interleukin-17 mRNA expression in blood and CSF mononuclear cells is augmented in multiple sclerosis. Mult Scler, 1999. 5(2): p. 101-104.

115. Haas J., Hug A., Viehover A., Fritzsching B., Falk C.S., Filser A., et al., Reduced suppressive effect of $C D 4+C D 25$ high regulatory $T$ cells on the $T$ cell immune response against myelin oligodendrocyte glycoprotein in patients with multiple sclerosis. Eur J Immunol, 2005. 35(11): p. 3343-3352.

116. Venken K., Hellings N., Hensen K., Rummens J.L., Medaer R., D'Hooghe M.B., et al., Secondary progressive in contrast to relapsing- remitting multiple sclerosis patients show a normal $\mathrm{CD} 4+\mathrm{CD} 25+$ regulatory $\mathrm{T}$-cell function and FOXP3 expression. J Neurosci Res, 2006. 83(8): p. 1432-1446.

117. Viglietta V., Baecher-Allan C., Weiner H.L., and Hafler D.A., Loss of functional suppression by $C D 4+C D 25+$ regulatory $T$ cells in patients with multiple sclerosis. J Exp Med, 2004. 199(7): p. 971-979.

118. Dalla Libera D., Di Mitri D., Bergami A., Centonze D., Gasperini C., Grasso M.G., et al., $T$ regulatory cells are markers of disease activity in multiple sclerosis patients. PLoS One, 2011. 6(6): p. e21386.

119. Edstrom M., Mellergard J., Mjosberg J., Jenmalm M., Vrethem M., Press R., et al., Transcriptional characteristics of $\mathrm{CD} 4+\mathrm{T}$ cells in multiple sclerosis: relative lack of suppressive populations in blood. Mult Scler, 2011. 17(1): p. 57-66.

120. Borsellino G., Kleinewietfeld M., Di Mitri D., Sternjak A., Diamantini A., Giometto R., et al., Expression of ectonucleotidase CD39 by Foxp3+ Treg cells: hydrolysis of extracellular ATP and immune suppression. Blood, 2007. 110(4): p. 1225-1232.

121. Fletcher J.M., Lonergan R., Costelloe L., Kinsella K., Moran B., O'Farrelly C., et al., CD39+Foxp3+ regulatory $T$ Cells suppress pathogenic Th17 cells and are impaired in multiple sclerosis. J Immunol, 2009. 183(11): p. 7602-7610.

122. Peelen E., Damoiseaux J., Smolders J., Knippenberg S., Menheere P., Cohen Tervaert J.W., et al., Th17 expansion in MS patients is counterbalanced by an expanded CD39+ regulatory $T$ cell population during remission but not during relapse. J Neuroimmunol, 2011. 240-241: p. 97-103.

123. Frischer J.M., Bramow S., Dal-Bianco A., Lucchinetti C.F., Rauschka H., Schmidbauer M., et al., The relation between inflammation and neurodegeneration in multiple sclerosis brains. Brain, 2009. 132(Pt 5): p. 1175-1189.

124. Junker A., Ivanidze J., Malotka J., Eiglmeier I., Lassmann H., Wekerle $H_{\text {., }}$ et al., Multiple sclerosis: T-cell receptor expression in distinct brain regions. Brain, 2007. 130(Pt 11): p. 2789-2799. 
125. Quintana F.J., Farez M.F., Izquierdo G., Lucas M., Cohen I.R., and Weiner H.L., Antigen microarrays identify CNS-produced autoantibodies in RRMS. Neurology, 2012. 78(8): p. 532-539.

126. Cohen J.A., Barkhof F., Comi G., Hartung H.P., Khatri B.O., Montalban X., et al., Oral fingolimod or intramuscular interferon for relapsing multiple sclerosis. N Engl J Med, 2010. 362(5): p. 402-415.

127. Noronha A., Toscas A., and Jensen M.A., Interferon beta decreases $T$ cell activation and interferon gamma production in multiple sclerosis. J Neuroimmunol, 1993. 46(1-2): p. 145-153.

128. Polman C., Schellekens H., and Killestein J., Neutralizing antibodies to interferon-beta may persist after cessation of therapy: what impact could they have? Mult Scler, 2006. 12(3): p. 245-246.

129. Coles A.J., Compston D.A., Selmaj K.W., Lake S.L., Moran S., Margolin D.H., et al., Alemtuzumab vs. interferon beta- $1 \mathrm{a}$ in early multiple sclerosis. N Engl J Med, 2008. 359(17): p. 1786-1801.

130. Hauser S.L., Waubant E., Arnold D.L., Vollmer T., Antel J., Fox R.J., et al., B-cell depletion with rituximab in relapsing-remitting multiple sclerosis. N Engl J Med, 2008. 358(7): p. 676-688.

131. Smolders J., Damoiseaux J., Menheere P., and Hupperts R., Vitamin D as an immune modulator in multiple sclerosis, a review. J Neuroimmunol, 2008. 194(1-2): p. 7-17.

132. Smolders J., Peelen E., Thewissen M., Menheere P., Cohen Tervaert J.W., Hupperts R., et al., The relevance of vitamin $D$ receptor gene polymorphisms for vitamin $D$ research in multiple sclerosis. Autoimmun Rev, 2009. 8(7): p. 621-626.

133. Munger K.L., Levin L.I., Hollis B.W., Howard N.S., and Ascherio A., Serum 25hydroxyvitamin $D$ levels and risk of multiple sclerosis. JAMA, 2006. 296(23): p. 2832-2838.

134. Wingerchuk D.M., Lesaux J., Rice G.P., Kremenchutzky M., and Ebers G.C., A pilot study of oral calcitriol (1,25-dihydroxyvitamin D3) for relapsing-remitting multiple sclerosis. J Neurol Neurosurg Psychiatry, 2005. 76(9): p. 1294-1296.
135. Dorr J., Ohlraun S., Skarabis H., and Paul F., Efficacy of Vitamin D Supplementation in Multiple Sclerosis (EVIDIMS Trial): study protocol for a randomized controlled trial. Trials, 2012. 13: p. 15.

136. Smolders J., Hupperts R., Barkhof F., Grimaldi L.M., Holmoy T., Killestein J., et al., Efficacy of vitamin D3 as add-on therapy in patients with relapsing-remitting multiple sclerosis receiving subcutaneous interferon beta-1a: a Phase II, multicenter, double-blind, randomized, placebo-controlled trial. J Neurol Sci, 2011. 311(1-2): p. 44-49.

137. Lips P., Vitamin D physiology. Prog Biophys Mol Biol, 2006. 92(1): p. 4-8.

138. Dusso A.S., Brown A.J., and Slatopolsky E., Vitamin D. Am J Physiol Renal Physiol, 2005. 289(1): p. F8-28.

139. Jongen M.J., van der Vijgh W.J., Lips P., and Netelenbos J.C., Measurement of vitamin D metabolites in anephric subjects. Nephron, 1984. 36(4): p. 230-234.

140. Zehnder D., Bland R., Williams M.C., McNinch R.W., Howie A.J., Stewart P.M., et al., Extrarenal expression of 25-hydroxyvitamin d(3)-1 alpha-hydroxylase. J Clin Endocrinol Metab, 2001. 86(2): p. 888-894.

141. Holick M.F., Vitamin D deficiency. N Engl J Med, 2007. 357(3): p. 266-281.

142. Holick M.F., Binkley N.C., Bischoff-Ferrari H.A., Gordon C.M., Hanley D.A., Heaney R.P., et al., Evaluation, treatment, and prevention of vitamin D deficiency: an Endocrine Society clinical practice guideline. J Clin Endocrinol Metab, 2011. 96(7): p. 1911-1930.

143. Institute of Medicine, Food and Nutrition Board., Dietary Reference Intakes for Calcium and Vitamin D. 2010, National Academy Press: Washington, DC.

144. Ross A.C., The 2011 report on dietary reference intakes for calcium and vitamin D. Public Health Nutr, 2011. 14(5): p. 938-939.

145. Vieth R., Vitamin D supplementation, 25hydroxyvitamin D concentrations, and safety. Am J Clin Nutr, 1999. 69(5): p. 842-856.

146. Baeke F., Korf H., Overbergh L., van Etten E., Verstuyf A., Gysemans C., et al., Human T lymphocytes are direct targets of 1,25dihydroxyvitamin D3 in the immune system. 
J Steroid Biochem Mol Biol, 2010. 121(1-2): p. 221-227.

147. Peelen E., Knippenberg S., Muris A.H., Thewissen M., Smolders J., Cohen Tervaert J.W., et al., Effects of vitamin $D$ on the peripheral adaptive immune system: a review. Autoimmun Rev, 2011. 10(12): p. 733-743.

148. Chen S., Sims G.P., Chen X.X., Gu Y.Y., and Lipsky P.E., Modulatory effects of 1,25dihydroxyvitamin D3 on human B cell differentiation. J Immunol, 2007. 179(3): p. 16341647.

149. Penna G., Amuchastegui S., Giarratana N., Daniel K.C., Vulcano M., Sozzani S., et al., 1,25-Dihydroxyvitamin D3 selectively modulates tolerogenic properties in myeloid but not plasmacytoid dendritic cells. J Immunol, 2007. 178(1): p. 145-153.

150. Provvedini D.M., Tsoukas C.D., Deftos L.J., and Manolagas S.C., 1,25-dihydroxyvitamin D3 receptors in human leukocytes. Science, 1983. 221(4616): p. 1181-1183.

151. Veldman C.M., Cantorna M.T., and DeLuca H.F., Expression of 1,25-dihydroxyvitamin $D(3)$ receptor in the immune system. Arch Biochem Biophys, 2000. 374(2): p. 334-338.

152. Penna G. and Adorini L., 1 Alpha,25dihydroxyvitamin D3 inhibits differentiation, maturation, activation, and survival of dendritic cells leading to impaired alloreactive $T$ cell activation. J Immunol, 2000. 164(5): p. 2405-2411.

153. van Halteren A.G., van Etten E., de Jong E.C., Bouillon R., Roep B.O., and Mathieu C., Redirection of human autoreactive T-cells Upon interaction with dendritic cells modulated by TX527, an analog of 1,25 dihydroxyvitamin D(3). Diabetes, 2002. 51(7): p. 2119-2125.

154. Correale J., Ysrraelit M.C., and Gaitan M.I., Immunomodulatory effects of Vitamin $D$ in multiple sclerosis. Brain, 2009. 132(Pt 5): p. 1146-1160.

155. Fritsche J., Mondal K., Ehrnsperger A., Andreesen R., and Kreutz M., Regulation of 25hydroxyvitamin D3-1 alpha-hydroxylase and production of 1 alpha,25-dihydroxyvitamin D3 by human dendritic cells. Blood, 2003. 102(9): p. 3314-3316.

156. Gottfried E., Rehli M., Hahn J., Holler E., Andreesen R., and Kreutz M., Monocyte- derived cells express CYP27A1 and convert vitamin D3 into its active metabolite. Biochem Biophys Res Commun, 2006. 349(1): p. 209-213.

157. Heine G., Niesner U., Chang H.D., Steinmeyer A., Zugel U., Zuberbier T., et al., 1,25dihydroxyvitamin $\mathrm{D}(3)$ promotes IL-10 production in human B cells. Eur J Immunol, 2008. 38(8): p. 2210-2218.

158. Sigmundsdottir H., Pan J., Debes G.F., Alt C., Habtezion A., Soler D., et al., DCs metabolize sunlight-induced vitamin D3 to 'program' T cell attraction to the epidermal chemokine CCL27. Nat Immunol, 2007. 8(3): p. 285-293.

159. Spach K.M. and Hayes C.E., Vitamin D3 confers protection from autoimmune encephalomyelitis only in female mice. J Immunol, 2005. 175(6): p. 4119-4126.

160. Cantorna M.T., Hayes C.E., and DeLuca H.F., 1,25-Dihydroxyvitamin D3 reversibly blocks the progression of relapsing encephalomyelitis, a model of multiple sclerosis. Proc Natl Acad Sci U S A, 1996. 93(15): p. 7861-7864.

161. Lemire J.M. and Archer D.C., 1,25dihydroxyvitamin D3 prevents the in vivo induction of murine experimental autoimmune encephalomyelitis. J Clin Invest, 1991. 87(3): p. 1103-1107.

162. Peterson C.A. and Heffernan M.E., Serum tumor necrosis factor-alpha concentrations are negatively correlated with serum $25(\mathrm{OH}) \mathrm{D}$ concentrations in healthy women. J Inflamm (Lond), 2008. 5: p. 10.

163. Shea M.K., Booth S.L., Massaro J.M., Jacques P.F., D'Agostino R.B. Sr., Dawson-Hughes B., et al., Vitamin $\mathrm{K}$ and vitamin $\mathrm{D}$ status: associations with inflammatory markers in the Framingham Offspring Study. Am J Epidemiol, 2008. 167(3): p. 313-320.

164. Vilarrasa N., Vendrell J., Maravall J., Elio I., Solano E., San Jose P., et al., Is plasma $25(\mathrm{OH}) \mathrm{D}$ related to adipokines, inflammatory cytokines and insulin resistance in both a healthy and morbidly obese population? Endocrine, 2010. 38(2): p. 235-242.

165. Jorde R., Sneve M., Torjesen P.A., Figenschau Y., Goransson L.G., and Omdal R., No effect of supplementation with cholecalciferol on cytokines and markers of inflammation in 
overweight and obese subjects. Cytokine, 2010. 50(2): p. 175-180.

166. Pittas A.G., Harris S.S., Stark P.C., and Dawson-Hughes B., The effects of calcium and vitamin D supplementation on blood glucose and markers of inflammation in nondiabetic adults. Diabetes Care, 2007. 30(4): p. 980-986.

167. Prietl B., Pilz S., Wolf M., Tomaschitz A., Obermayer-Pietsch B., Graninger W., et al., Vitamin D supplementation and regulatory $T$ cells in apparently healthy subjects: vitamin D treatment for autoimmune diseases? Isr Med Assoc J, 2010. 12(3): p. 136-139.

168. Kimball S., Vieth R., Dosch H.M., Bar-Or A., Cheung R., Gagne D., et al., Cholecalciferol plus calcium suppresses abnormal PBMC reactivity in patients with multiple sclerosis. J Clin Endocrinol Metab, 2011. 96(9): p. 28262834.

169. Thudi A., Yin S., Wandstrat A.E., Li Q.Z., and Olsen N.J., Vitamin D levels and disease status in Texas patients with systemic lupus erythematosus. Am J Med Sci, 2008. 335(2): p. 99-104.

170. Teichmann J., Voglau M.J., and Lange U., Antibodies to human tissue transglutaminase and alterations of vitamin D metabolism in ankylosing spondylitis and psoriatic arthritis. Rheumatol Int, 2010. 30(12): p. 1559-1563.

171. Do J.E., Kwon S.Y., Park S., and Lee E.S., Effects of vitamin $D$ on expression of Tolllike receptors of monocytes from patients with Behcet's disease. Rheumatology (Oxford), 2008. 47(6): p. 840-848.

172. Hamzaoui K., Ben Dhifallah I., Karray E., Sassi F.H., and Hamzaoui A., Vitamin D modulates peripheral immunity in patients with Behcet's disease. Clin Exp Rheumatol, 2010. 28(4 Suppl 60): p. S50-57.

173. Falkenbach A. and Sedlmeyer A., Travel to sunny countries is associated with changes in immunological parameters. Photodermatol Photoimmunol Photomed, 1997. 13(4): p. 139-142.

174. Szodoray P., Tarr T., Bazso A., Poor G., Szegedi G., and Kiss E., The immunopathological role of vitamin $D$ in patients with SLE: data from a single centre registry in Hungary. Scand J Rheumatol, 2011. 40(2): p. 122-126.

175. Hajas A., Sandor J., Csathy L., Csipo I., Barath S., Paragh G., et al., Vitamin D insufficiency in a large MCTD population. Autoimmun Rev, 2011. 10(6): p. 317-324.

176. Barnes M.S., Horigan G., Cashman K.D., Hill T.R., Forsythe L.K., Lucey A.J., et al., Maintenance of wintertime vitamin $D$ status with cholecalciferol supplementation is not associated with alterations in serum cytokine concentrations among apparently healthy younger or older adults. J Nutr, 2011. 141(3): p. 476-481.

177. Smolders J., Vogt M., and Hupperts R. Vitamin D3 supplementation does not affect circulating osteopontin levels in MS. JCEM. 2011.

178. Knippenberg S., Smolders J., Thewissen M., Peelen E., Cohen Tervaert J.W., Hupperts R., et al., Effect of vitamin $D(3)$ supplementation on peripheral $\mathrm{B}$ cell differentiation and isotype switching in patients with multiple sclerosis. Mult Scler, 2011. 17(12): p. 1418-1423.

179. Zittermann A., Dembinski J., and Stehle P., Low vitamin $D$ status is associated with low cord blood levels of the immunosuppressive cytokine interleukin-10. Pediatr Allergy Immunol, 2004. 15(3): p. 242-246.

180. Mahon B.D., Gordon S.A., Cruz J., Cosman F., and Cantorna M.T., Cytokine profile in patients with multiple sclerosis following vitamin D supplementation. J Neuroimmunol, 2003. 134(1-2): p. 128-132.

181. Adams J.S., Ren S., Liu P.T., Chun R.F., Lagishetty V., Gombart A.F., et al., Vitamin ddirected rheostatic regulation of monocyte antibacterial responses. J Immunol, 2009. 182(7): p. 4289-4295.

182. Khoo A.L., Chai L.Y., Koenen H.J., Sweep F.C., Joosten I., Netea M.G., et al., Regulation of cytokine responses by seasonality of vitamin D status in healthy individuals. Clin Exp Immunol, 2011. 164(1): p. 72-79.

183. Bendix-Struve M., Bartels L.E., Agnholt J., Dige A., Jorgensen S.P., and Dahlerup J.F., Vitamin D3 treatment of Crohn's disease patients increases stimulated T cell IL- 6 production and proliferation. Aliment Pharmacol Ther, 2010. 32(11-12): p. 1364-1372. 
184. Heine G., Drozdenko G., Lahl A., Unterwalder N., Mei H., Volk H.D., et al., Efficient tetanus toxoid immunization on vitamin $\mathrm{D}$ supplementation. Eur J Clin Nutr, 2011. 65(3): p. 329-334.

185. Martineau A.R., Wilkinson R.J., Wilkinson K.A., Newton S.M., Kampmann B., Hall B.M., et al., A single dose of vitamin $D$ enhances immunity to mycobacteria. Am J Respir Crit Care Med, 2007. 176(2): p. 208-213.

186. Mosayebi G., Ghazavi A., Ghasami K., Jand Y., and Kokhaei P., Therapeutic effect of vitamin D3 in multiple sclerosis patients. Immunol Invest, 2011. 40(6): p. 627-639.

187. Allen A.C., Kelly S., Basdeo S.A., Kinsella K., Mulready K.J., Mills K.H., et al., A pilot study of the immunological effects of high-dose vitamin $D$ in healthy volunteers. Mult Scler, 2012. 18(12): p. 1797-1800.

188. Ben-Zvi I., Aranow C., Mackay M., Stanevsky A., Kamen D.L., Marinescu L.M., et al., The impact of vitamin D on dendritic cell function in patients with systemic lupus erythematosus. PLoS One, 2010. 5(2): p. e9193.

189. Royal W. 3rd, Mia Y., Li H., and Naunton K., Peripheral blood regulatory $\mathrm{T}$ cell measurements correlate with serum vitamin $\mathrm{D}$ levels in patients with multiple sclerosis. J Neuroimmunol, 2009. 213(1-2): p. 135-141.

190. Smolders J., Menheere P., Thewissen M., Peelen E., Cohen Tervaert J.W., Hupperts R., et al., Regulatory $T$ cell function correlates with serum 25-hydroxyvitamin $D$, but not with 1,25-dihydroxyvitamin D, parathyroid hormone and calcium levels in patients with relapsing remitting multiple sclerosis. J Steroid Biochem Mol Biol, 2010. 121(1-2): p. 243246.

191. Smolders J., Thewissen M., Peelen E., Menheere P., Cohen Tervaert J.W., Damoiseaux J., et al., Vitamin D status is positively correlated with regulatory $T$ cell function in patients with multiple sclerosis. PLoS One, 2009. 4(8): p. e6635.

192. Bock G., Prietl B., Mader J.K., Holler E., Wolf M., Pilz S., et al., The effect of vitamin D supplementation on peripheral regulatory $T$ cells and beta cell function in healthy humans: a randomized controlled trial. Diabetes Metab Res Rev, 2011. 27(8): p. 942-945.
193. Ritterhouse L.L., Crowe S.R., Niewold T.B., Kamen D.L., Macwana S.R., Roberts V.C., et al., Vitamin D deficiency is associated with an increased autoimmune response in healthy individuals and in patients with systemic lupus erythematosus. Ann Rheum Dis, 2011. 70(9): p. 1569-1574.

194. Bonakdar Z.S., Jahanshahifar L., Jahanshahifar F., and Gholamrezaei A., Vitamin $D$ deficiency and its association with disease activity in new cases of systemic lupus erythematosus. Lupus, 2011. 20(11): p. 11551160.

195. Kivity S., Agmon-Levin N., Zisappl M., Shapira Y., Nagy E.V., Danko K., et al., Vitamin $D$ and autoimmune thyroid diseases. Cell Mol Immunol, 2011. 8(3): p. 243-247.

196. Feser M., Derber L.A., Deane K.D., Lezotte D.C., Weisman M.H., Buckner J.H., et al., Plasma 25, $\mathrm{OH}$ vitamin D concentrations are not associated with rheumatoid arthritis (RA)-related autoantibodies in individuals at elevated risk for RA. J Rheumatol, 2009. 36(5): p. 943-946.

197. Baeke F., Takiishi T., Korf H., Gysemans C., and Mathieu C., Vitamin D: modulator of the immune system. Curr Opin Pharmacol, 2010. 10(4): p. 482-496.

198. Lai J.K., Lucas R.M., Banks E., and Ponsonby A.L., Variability in vitamin $D$ assays impairs clinical assessment of vitamin D status. Intern Med J, 2012. 42(1): p. 43-50.

199. Soilu-Hanninen M., Airas L., Mononen I., Heikkila A., Viljanen M., and Hanninen A., 25Hydroxyvitamin $D$ levels in serum at the onset of multiple sclerosis. Mult Scler, 2005. 11(3): p. 266-271.

200. Soilu-Hanninen M., Laaksonen M., Laitinen I., Eralinna J.P., Lilius E.M., and Mononen I., A longitudinal study of serum 25hydroxyvitamin $D$ and intact parathyroid hormone levels indicate the importance of vitamin $\mathrm{D}$ and calcium homeostasis regulation in multiple sclerosis. J Neurol Neurosurg Psychiatry, 2008. 79(2): p. 152-157.

201. Salzer J., Bistrom M., and Sundstrom P., Vitamin D and multiple sclerosis: where do we go from here? Expert Rev Neurother, 2014. 14(1): p. 9-18. 
202. Weinstock-Guttman B., Zivadinov R., Qu J., Cookfair D., Duan X., Bang E., et al., Vitamin $D$ metabolites are associated with clinical and MRI outcomes in multiple sclerosis patients. Journal of neurology, neurosurgery, and psychiatry, 2011. 82(2): p. 189-195.

203. D'Hooghe M.B., Haentjens P., Nagels G., Garmyn M., and De Keyser J., Sunlight exposure and sun sensitivity associated with disability progression in multiple sclerosis. Multiple sclerosis, 2012. 18(4): p. 451-459.

204. McDowell T.Y., Amr S., Culpepper W.J., Langenberg P., Royal W., Bever C., et al., Sun exposure, vitamin $D$ and age at disease onset in relapsing multiple sclerosis. Neuroepidemiology, 2011. 36(1): p. 39-45.

205. Gelfand J.M., Cree B.A., McElroy J., Oksenberg J., Green R., Mowry E.M., et al., Vitamin $D$ in African Americans with multiple sclerosis. Neurology, 2011. 76(21): p. 1824-1830.

206. Ascherio A., Munger K.L., White R., Kochert K., Simon K.C., Polman C.H., et al., Vitamin D as an early predictor of multiple sclerosis activity and progression. JAMA Neurol, 2014. 71(3): p. 306-314.

207. Hirst C., Ingram G., Pearson O., Pickersgill T., Scolding N., and Robertson N., Contribution of relapses to disability in multiple sclerosis. J Neurol, 2008. 255(2): p. 280-287.

208. Lublin F.D., Baier M., and Cutter G., Effect of relapses on development of residual deficit in multiple sclerosis. Neurology, 2003. 61(11): p. 1528-1532.

209. Kurtzke J.F., Rating neurologic impairment in multiple sclerosis: an expanded disability status scale (EDSS). Neurology, 1983. 33(11): p. $1444-1452$.

210. Non WMO Research. Central Commitee on Research Involving Human Subjects. 2015. Available from http://www.ccmo.nl/en/nonwmo-research

211. Lublin F.D. and Reingold S.C., Defining the clinical course of multiple sclerosis: results of an international survey. National Multiple Sclerosis Society (USA) Advisory Committee on Clinical Trials of New Agents in Multiple Sclerosis. Neurology, 1996. 46(4): p. 907-911.

212. Knippenberg S. and Damoiseaux J., 25hydroxyvitamin D level prediction models - is further optimization feasible? Neuroepidemiology, 2012. 39(2): p. 94-95.

213. Saltyte Benth J., Myhr K.M., Loken-Amsrud K.I., Beiske A.G., Bjerve K.S., Hovdal H., et al., Modelling and prediction of 25hydroxyvitamin D levels in Norwegian relapsing-remitting multiple sclerosis patients. Neuroepidemiology, 2012. 39(2): p. 84-93.

214. Brown M.G., Kirby S., Skedgel C., Fisk J.D., Murray T.J., Bhan V., et al., How effective are disease-modifying drugs in delaying progression in relapsing-onset MS? Neurology, 2007. 69(15): p. 1498-1507.

215. Loken-Amsrud K.I., Holmoy T., Bakke S.J., Beiske A.G., Bjerve K.S., Bjornara B.T., et al., Vitamin $D$ and disease activity in multiple sclerosis before and during interferon-beta treatment. Neurology, 2012. 79(3): p. 267273.

216. Confavreux C., Vukusic S., Moreau T., and Adeleine P., Relapses and progression of disability in multiple sclerosis. N Engl J Med, 2000. 343(20): p. 1430-1438.

217. Etemadifar M., Janghorbani M., and Shaygannejad V., Comparison of Betaferon, Avonex, and Rebif in treatment of relapsingremitting multiple sclerosis. Acta Neurol Scand, 2006. 113(5): p. 283-287.

218. Nikfar S., Rahimi R., and Abdollahi M., A meta-analysis of the efficacy and tolerability of interferon-beta in multiple sclerosis, overall and by drug and disease type. Clin Ther, 2010. 32(11): p. 1871-1888.

219. Shirani A., Zhao Y., Karim M.E., Evans C., Kingwell E., van der Kop M.L., et al., Association between use of interferon beta and progression of disability in patients with relapsing-remitting multiple sclerosis. JAMA, 2012. 308(3): p. 247-256.

220. Panitch H., Miller A., Paty D., Weinshenker B., and North American Study Group on Interferon beta-1b in Secondary Progressive MS, Interferon beta-1b in secondary progressive MS: results from a 3-year controlled study. Neurology, 2004. 63(10): p. 1788-1795.

221. Ford C., Goodman A.D., Johnson K., Kachuck N., Lindsey J.W., Lisak R., et al., Continuous long-term immunomodulatory therapy in relapsing multiple sclerosis: results from the 15-year analysis of the US prospective open- 
label study of glatiramer acetate. Mult Scler, 2010. 16(3): p. 342-350.

222. O'Connor P., Goodman A., Kappos L., Lublin F., Polman C., Rudick R.A., et al., Long-term safety and effectiveness of natalizumab redosing and treatment in the STRATA MS Study. Neurology, 2014. 83(1): p. 78-86.

223. Munger K.L., Kochert K., Simon K.C., Kappos L., Polman C.H., Freedman M.S., et al., Molecular mechanism underlying the impact of vitamin D on disease activity of MS. Ann Clin Transl Neurol, 2014. 1(8): p. 605-617.

224. Lin R., Taylor B.V., Charlesworth J., van der Mei I., Blizzard L., Stewart N., et al., Modulating effects of WT1 on interferon-betavitamin D association in MS. Acta Neurol Scand, 2014.

225. Stewart N., Simpson S., Jr., van der Mei I., Ponsonby A.L., Blizzard L., Dwyer T., et al., Interferon-beta and serum 25-hydroxyvitamin $\mathrm{D}$ interact to modulate relapse risk in MS. Neurology, 2012. 79(3): p. 254-260.

226. Waschbisch A., Sanderson N., Krumbholz M., Vlad G., Theil D., Schwab S., et al., Interferon Beta and vitamin d synergize to induce immunoregulatory receptors on peripheral blood monocytes of multiple sclerosis patients. PLoS One, 2014. 9(12): p. e115488.

227. Rosjo E., Myhr K.M., Loken-Amsrud K.I., Bakke S.J., Beiske A.G., Bjerve K.S., et al., Vitamin $D$ status and effect of interferonbetala treatment on MRI activity and serum inflammation markers in relapsing-remitting multiple sclerosis. J Neuroimmunol, 2015. 280: p. 21-28.

228. Lublin F.D., Reingold S.C., Cohen J.A., Cutter G.R., Sorensen P.S., Thompson A.J., et al., Defining the clinical course of multiple sclerosis: the 2013 revisions. Neurology, 2014. 83(3): p. 278-286.

229. Weinshenker B.G., Bass B., Rice G.P., Noseworthy J., Carriere W., Baskerville J., et al., The natural history of multiple sclerosis: a geographically based study. 2. Predictive value of the early clinical course. Brain, 1989. 112 ( Pt 6): p. 1419-1428.

230. Leray E., Yaouanq J., Le Page E., Coustans M., Laplaud D., Oger J., et al., Evidence for a twostage disability progression in multiple sclerosis. Brain, 2010. 133(Pt 7): p. 1900-1913.
231. Tremlett H., Paty D., and Devonshire V., Disability progression in multiple sclerosis is slower than previously reported. Neurology, 2006. 66(2): p. 172-177.

232. Kamm C.P., Uitdehaag B.M., and Polman C.H., Multiple sclerosis: current knowledge and future outlook. Eur Neurol, 2014. 72(34): p. 132-141.

233. Ontaneda D., Fox R.J., and Chataway J., Clinical trials in progressive multiple sclerosis: lessons learned and future perspectives. Lancet Neurol, 2015. 14(2): p. 208-223.

234. Fitzgerald K.C., Munger K.L., Kochert K., Arnason B.G., Comi G., Cook S., et al., Association of Vitamin D Levels With Multiple Sclerosis Activity and Progression in Patients Receiving Interferon Beta-1b. JAMA Neurol, 2015. 72(12): p. 1458-1465.

235. Muris A.H., Smolders J., Rolf L., Klinkenberg L.J.J., van der Linden N., Meex S., et al., Vitamin D Status Does Not Affect Disability Progression of Patients with Multiple Sclerosis over Three Year Follow-Up. PLoS One, 2016. 11(6): e0156122.

236. Koch M., Kingwell E., Rieckmann P., Tremlett $\mathrm{H}$., and UBC MS Clinic Neurologists, The natural history of secondary progressive multiple sclerosis. J Neurol Neurosurg Psychiatry, 2010. 81(9): p. 1039-1043.

237. Edan G., Kappos L., Montalban X., Polman C.H., Freedman M.S., Hartung H.P., et al., Long-term impact of interferon beta-1b in patients with CIS: 8-year follow-up of BENEFIT. J Neurol Neurosurg Psychiatry, 2014. 85(11): p. 1183-1189.

238. Zastepa E., Fitz-Gerald L., Hallett M., Antel J., Bar-Or A., Baranzini S., et al., Naive CD4 Tcell activation identifies MS patients having rapid transition to progressive MS. Neurology, 2014. 82(8): p. 681-690.

239. El-behi M., Rostami A., and Ciric B., Current views on the roles of Th1 and Th17 cells in experimental autoimmune encephalomyelitis. J Neuroimmune Pharmacol, 2010. 5(2): p. 189-197.

240. Ferber I.A., Brocke S., Taylor-Edwards C., Ridgway W., Dinisco C., Steinman L., et al., Mice with a disrupted IFN-gamma gene are susceptible to the induction of experimental 
autoimmune encephalomyelitis (EAE). J Immunol, 1996. 156(1): p. 5-7.

241. Komiyama Y., Nakae S., Matsuki T., Nambu

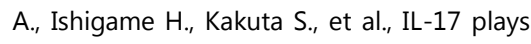
an important role in the development of experimental autoimmune encephalomyelitis. J Immunol, 2006. 177(1): p. 566-573.

242. Cua D.J., Sherlock J., Chen Y., Murphy C.A., Joyce B., Seymour B., et al., Interleukin-23 rather than interleukin-12 is the critical cytokine for autoimmune inflammation of the brain. Nature, 2003. 421(6924): p. 744-748.

243. Segal B.M., Dwyer B.K., and Shevach E.M., An interleukin (IL)-10/IL-12 immunoregulatory circuit controls susceptibility to autoimmune disease. J Exp Med, 1998. 187(4): p. 537-546.

244. El-Behi M., Ciric B., Dai H., Yan Y., Cullimore M., Safavi F., et al., The encephalitogenicity of $\mathrm{T}(\mathrm{H}) 17$ cells is dependent on IL-1- and IL23-induced production of the cytokine GMCSF. Nat Immunol, 2011. 12(6): p. 568-575.

245. McGeachy M.J., Bak-Jensen K.S., Chen Y., Tato C.M., Blumenschein W., McClanahan T., et al., TGF-beta and IL- 6 drive the production of $\mathrm{IL}-17$ and IL-10 by $\mathrm{T}$ cells and restrain $\mathrm{T}(\mathrm{H})-17$ cell-mediated pathology. Nat Immunol, 2007. 8(12): p. 1390-1397.

246. Hamilton J.A., Colony-stimulating factors in inflammation and autoimmunity. Nat Rev Immunol, 2008. 8(7): p. 533-544.

247. McQualter J.L., Darwiche R., Ewing C., Onuki M., Kay T.W., Hamilton J.A., et al., Granulocyte macrophage colony-stimulating factor: a new putative therapeutic target in multiple sclerosis. J Exp Med, 2001. 194(7): p. 873882.

248. Ponomarev E.D., Shriver L.P., Maresz K., Pedras-Vasconcelos J., Verthelyi D., and Dittel B.N., GM-CSF production by autoreactive $T$ cells is required for the activation of microglial cells and the onset of experimental autoimmune encephalomyelitis. J Immunol, 2007. 178(1): p. 39-48.

249. Campbell I.K., Rich M.J., Bischof R.J., Dunn A.R., Grail D., and Hamilton J.A., Protection from collagen-induced arthritis in granulocyte-macrophage colony-stimulating factordeficient mice. J Immunol, 1998. 161(7): p. 3639-3644.
250. Sonderegger I., Iezzi G., Maier R., Schmitz N., Kurrer M., and Kopf M., GM-CSF mediates autoimmunity by enhancing IL-6-dependent Th17 cell development and survival. J Exp Med, 2008. 205(10): p. 2281-2294.

251. Sheng W., Yang F., Zhou Y., Yang H., Low P.Y., Kemeny D.M., et al., STAT5 programs a distinct subset of GM-CSF-producing $T$ helper cells that is essential for autoimmune neuroinflammation. Cell Res, 2014. 24(12): p. 1387-1402.

252. Bhattacharya P., Gopisetty A., Ganesh B.B., Sheng J.R., and Prabhakar B.S., GM-CSFinduced, bone-marrow-derived dendritic cells can expand natural Tregs and induce adaptive Tregs by different mechanisms. J Leukoc Biol, 2011. 89(2): p. 235-249.

253. Zou T., Satake A., Ojha P., and Kambayashi T., Cellular therapies supplement: the role of granulocyte macrophage colony-stimulating factor and dendritic cells in regulatory T-cell homeostasis and expansion. Transfusion, 2011. 51 Suppl 4: p. 160S-168S.

254. Hartmann F.J., Khademi M., Aram J., Ammann S., Kockum I., Constantinescu C., et al., Multiple sclerosis-associated IL2RA polymorphism controls GM-CSF production in human TH cells. Nat Commun, 2014. 5: p. 5056.

255. Young A., Linehan E., Hams E., O'Hara Hall A.C., McClurg A., Johnston J.A., et al., Cutting edge: suppression of GM-CSF expression in murine and human T cells by IL-27. J Immunol, 2012. 189(5): p. 2079-2083.

256. Carrieri P.B., Provitera V., De Rosa T., Tartaglia G., Gorga F., and Perrella O., Profile of cerebrospinal fluid and serum cytokines in patients with relapsing-remitting multiple sclerosis: a correlation with clinical activity. Immunopharmacol Immunotoxicol, 1998. 20(3): p. 373-382.

257. Vrethem M., Kvarnstrom M., Stenstam J., Cassel P., Gustafsson M., Landtblom A.M., et al., Cytokine mapping in cerebrospinal fluid and blood in multiple sclerosis patients without oligoclonal bands. Mult Scler, 2012. 18(5): p. 669-673.

258. Hellings N., Baree M., Verhoeven C., D'Hooghe M B., Medaer R., Bernard C.C., et al., T-cell reactivity to multiple myelin anti- 
gens in multiple sclerosis patients and healthy controls. J Neurosci Res, 2001. 63(3): p. 290-302.

259. Brickshawana A., Hinson S.R., Romero M.F., Lucchinetti C.F., Guo Y., Buttmann M., et al., Investigation of the KIR4.1 potassium channel as a putative antigen in patients with multiple sclerosis: a comparative study. Lancet Neurol, 2014. 13(8): p. 795-806.

260. Romio M., Reinbeck B., Bongardt S., Huls S., Burghoff S., and Schrader J., Extracellular purine metabolism and signaling of CD73derived adenosine in murine Treg and Teff cells. Am J Physiol Cell Physiol, 2011. 301(2): p. C530-539.

261. Alam M.S., Kurtz C.C., Rowlett R.M., Reuter B.K., Wiznerowicz E., Das S., et al., CD73 is expressed by human regulatory $\mathrm{T}$ helper cells and suppresses proinflammatory cytokine production and Helicobacter felisinduced gastritis in mice. J Infect Dis, 2009. 199(4): p. 494-504.

262. Bours M.J., Swennen E.L., Di Virgilio F., Cronstein B.N., and Dagnelie P.C., Adenosine 5 '-triphosphate and adenosine as endogenous signaling molecules in immunity and inflammation. Pharmacol Ther, 2006. 112(2): p. 358-404.

263. Towers T.L. and Freedman L.P., Granulocytemacrophage colony-stimulating factor gene transcription is directly repressed by the vitamin D3 receptor. Implications for allosteric influences on nuclear receptor structure and function by a DNA element. J Biol Chem, 1998. 273(17): p. 10338-10348.

264. MacNeil I.A., Suda T., Moore K.W., Mosmann T.R., and Zlotnik A., IL-10, a novel growth cofactor for mature and immature $T$ cells. J Immunol, 1990. 145(12): p. 4167-4173.

265. Mosmann T.R., Schumacher J.H., Fiorentino D.F., Leverah J., Moore K.W., and Bond M.W., Isolation of monoclonal antibodies specific for IL-4, IL-5, IL-6, and a new Th2-specific cytokine (IL-10), cytokine synthesis inhibitory factor, by using a solid phase radioimmunoadsorbent assay. J Immunol, 1990. 145(9): p. 2938-2945.

266. Fujio K., Okamura T., and Yamamoto K., The Family of IL-10-secreting CD4+ T cells. Adv Immunol, 2010. 105: p. 99-130.
267. O'Garra A., Barrat F.J., Castro A.G., Vicari A., and Hawrylowicz C., Strategies for use of IL10 or its antagonists in human disease. Immunol Rev, 2008. 223: p. 114-131.

268. Rennick D.M., Fort M.M., and Davidson N.J., Studies with IL-10-/- mice: an overview. J Leukoc Biol, 1997. 61(4): p. 389-396.

269. Battaglia M., Gregori S., Bacchetta R., and Roncarolo M.G., Tr1 cells: from discovery to their clinical application. Semin Immunol, 2006. 18(2): p. 120-127.

270. Groux H. and Cottrez F., The complex role of interleukin-10 in autoimmunity. J Autoimmun, 2003. 20(4): p. 281-285.

271. Groux H., O'Garra A., Bigler M., Rouleau M., Antonenko S., de Vries J.E., et al., A CD4+ Tcell subset inhibits antigen-specific T-cell responses and prevents colitis. Nature, 1997. 389(6652): p. 737-742.

272. Beilke J.N., Kuhl N.R., Van Kaer L., and Gill R.G., NK cells promote islet allograft tolerance via a perforin-dependent mechanism. Nat Med, 2005. 11(10): p. 1059-1065.

273. Lund F.E., Cytokine-producing B lymphocytes-key regulators of immunity. Curr Opin Immunol, 2008. 20(3): p. 332-338.

274. Yu G., Xu X., Vu M.D., Kilpatrick E.D., and Li X.C., NK cells promote transplant tolerance by killing donor antigen-presenting cells. J Exp Med, 2006. 203(8): p. 1851-1858.

275. Llorente L., Zou W., Levy Y., Richaud-Patin Y., Wijdenes J., Alcocer-Varela J., et al., Role of interleukin 10 in the $B$ lymphocyte hyperactivity and autoantibody production of human systemic lupus erythematosus. J Exp Med, 1995. 181(3): p. 839-844.

276. Rousset F., Garcia E., Defrance T., Peronne C., Vezzio N., Hsu D.H., et al., Interleukin 10 is a potent growth and differentiation factor for activated human B lymphocytes. Proc Natl Acad Sci U S A, 1992. 89(5): p. 1890-1893.

277. Carter L.L. and Swain S.L., Single cell analyses of cytokine production. Curr Opin Immunol, 1997. 9(2): p. 177-182.

278. Sander B., Andersson J., and Andersson U., Assessment of cytokines by immunofluorescence and the paraformaldehyde-saponin procedure. Immunol Rev, 1991. 119: p. 6593. 
279. Jung T., Schauer U., Heusser C., Neumann C., and Rieger $C_{\text {., }}$ Detection of intracellular cytokines by flow cytometry. J Immunol Methods, 1993. 159(1-2): p. 197-207.

280. Prussin C., Cytokine flow cytometry: understanding cytokine biology at the single-cell level. J Clin Immunol, 1997. 17(3): p. 195-204.

281. Dinter A. and Berger E.G., Golgi-disturbing agents. Histochem Cell Biol, 1998. 109(5-6): p. 571-590.

282. Klausner R.D., Donaldson J.G., and Lippincott-Schwartz J., Brefeldin A: insights into the control of membrane traffic and organelle structure. J Cell Biol, 1992. 116(5): p. 1071-1080.

283. Mollenhauer H.H., Morre D.J., and Rowe L.D., Alteration of intracellular traffic by monensin; mechanism, specificity and relationship to toxicity. Biochim Biophys Acta, 1990. 1031(2): p. 225-246.

284. Tartakoff A.M., Perturbation of vesicular traffic with the carboxylic ionophore monensin. Cell, 1983. 32(4): p. 1026-1028.

285. Caraher E.M., Parenteau M., Gruber H., and Scott F.W., Flow cytometric analysis of intracellular IFN-gamma, IL-4 and IL-10 in CD3(+)4(+) T-cells from rat spleen. J Immunol Methods, 2000. 244(1-2): p. 29-40.

286. Nylander S. and Kalies I., Brefeldin A, but not monensin, completely blocks CD69 expression on mouse lymphocytes: efficacy of inhibitors of protein secretion in protocols for intracellular cytokine staining by flow cytometry. J Immunol Methods, 1999. 224(1-2): p. 69-76.

287. Konry T., Dominguez-Villar M., Baecher-Allan C., Hafler D.A., and Yarmush M.L., Dropletbased microfluidic platforms for single $T$ cell secretion analysis of IL-10 cytokine. Biosens Bioelectron, 2011. 26(5): p. 2707-2710.

288. Hicks S.W. and Machamer C.E., Golgi structure in stress sensing and apoptosis. Biochim Biophys Acta, 2005. 1744(3): p. 406-414.

289. Schuerwegh A.J., Stevens W.J., Bridts C.H., and De Clerck L.S., Evaluation of monensin and brefeldin A for flow cytometric determination of interleukin-1 beta, interleukin-6, and tumor necrosis factor-alpha in monocytes. Cytometry, 2001. 46(3): p. 172-176.
290. Oku M., Tanakura S., Uemura A., Sohda M., Misumi Y., Taniguchi M., et al., Novel cisacting element GASE regulates transcriptional induction by the Golgi stress response. Cell Struct Funct, 2011. 36(1): p. 1-12.

291. Souza A.C., Machado F.S., Celes M.R., Faria G., Rocha L.B., Silva J.S., et al., Mitochondrial damage as an early event of monensininduced cell injury in cultured fibroblasts L929. J Vet Med A Physiol Pathol Clin Med, 2005. 52(5): p. 230-237.

292. Schroder M., Endoplasmic reticulum stress responses. Cellular and Molecular Life Sciences, 2008. 65(6): p. 862-894.

293. Hemmer B., Archelos J.J., and Hartung H.P., New concepts in the immunopathogenesis of multiple sclerosis. Nat Rev Neurosci, 2002. 3(4): p. 291-301.

294. Venken K., Hellings N., Liblau R., and Stinissen $\mathrm{P}$., Disturbed regulatory $\mathrm{T}$ cell homeostasis in multiple sclerosis. Trends Mol Med, 2010. 16(2): p. 58-68.

295. Knippenberg S., Peelen E., Smolders J., Thewissen M., Menheere P., Cohen Tervaert J.W., et al., Reduction in IL-10 producing $B$ cells (Breg) in multiple sclerosis is accompanied by a reduced naive/memory Breg ratio during a relapse but not in remission. J Neuroimmunol, 2011. 239(1-2): p. 80-86.

296. Muris A.H., Damoiseaux J., and Smolders J., Monitoring in vivo immune modulation by vitamin $D$ in multiple sclerosis, in Handbook of Vitamin D in human health: prevention, treatment, and toxicity, R.R. Watson, Editor. 2013, Wageningen Academic Publishers: Wageningen, the Netherlands. p. 474-500.

297. Peelen E., Muris A.H., Damoiseaux J., Knippenberg S., Broens K., Smolders J., et al., GMCSF production by CD4+ T cells in MS patients: regulation by regulatory $T$ cells and vitamin D. J Neuroimmunol, 2015. 280: p. 3642.

298. Prietl B., Treiber G., Mader J.K., Hoeller E., Wolf M., Pilz S., et al., High-dose cholecalciferol supplementation significantly increases peripheral $\mathrm{CD} 4(+)$ Tregs in healthy adults without negatively affecting the frequency of other immune cells. Eur J Nutr, 2014. 53(3): p. 751-759. 
299. Muris A.H., Damoiseaux J., Smolders J., Cohen Tervaert J.W., Hupperts R., and Thewissen M., Intracellular IL-10 detection in T cells by flowcytometry: the use of protein transport inhibitors revisited. J Immunol Methods, 2012. 381(1-2): p. 59-65.

300. de Jager W., te Velthuis H., Prakken B.J., Kuis W., and Rijkers G.T., Simultaneous detection of 15 human cytokines in a single sample of stimulated peripheral blood mononuclear cells. Clin Diagn Lab Immunol, 2003. 10(1): p. 133-139.

301. Blair P.A., Norena L.Y., Flores-Borja F., Rawlings D.J., Isenberg D.A., Ehrenstein M.R., et al., CD19(+)CD24(hi)CD38(hi) B cells exhibit regulatory capacity in healthy individuals but are functionally impaired in systemic Lupus Erythematosus patients. Immunity, 2010. 32(1): p. 129-140.

302. Ashtari F., Toghianifar N., Zarkesh-Esfahani S.H., and Mansourian M., Short-Term Effect of High-Dose Vitamin D on the Level of Interleukin 10 in Patients with Multiple Sclerosis: A Randomized, Double-Blind, PlaceboControlled Clinical Trial. Neuroimmunomodulation, 2015.

303. Aivo J., Hanninen A., Ilonen J., and SoiluHanninen M., Vitamin D3 administration to MS patients leads to increased serum levels of latency activated peptide (LAP) of TGFbeta. J Neuroimmunol, 2015. 280: p. 12-15.

304. Golan D., Halhal B., Glass-Marmor L., StaunRam E., Rozenberg O., Lavi I., et al., Vitamin D supplementation for patients with multiple sclerosis treated with interferon-beta: a randomized controlled trial assessing the effect on flu-like symptoms and immunomodulatory properties. BMC Neurol, 2013. 13: p. 60.

305. Sotirchos E.S., Bhargava P., Eckstein C., Van Haren K., Baynes M., Ntranos A., et al., Safety and immunologic effects of high- vs lowdose cholecalciferol in multiple sclerosis. Neurology. 2016. 86(4): p. 382-390.

306. Toghianifar N., Ashtari F., Zarkesh-Esfahani S.H., and Mansourian M., Effect of high dose vitamin $D$ intake on interleukin-17 levels in multiple sclerosis: a randomized, doubleblind, placebo-controlled clinical trial. J Neuroimmunol, 2015. 285: p. 125-128.
307. Hedegaard C.J., Krakauer M., Bendtzen K., Lund H., Sellebjerg F., and Nielsen C.H., T helper cell type 1 (Th1), Th2 and Th17 responses to myelin basic protein and disease activity in multiple sclerosis. Immunology, 2008. 125(2): p. 161-169.

308. Kostic M., Stojanovic I., Marjanovic G., Zivkovic N., and Cvetanovic A., Deleterious versus protective autoimmunity in multiple sclerosis. Cell Immunol, 2015. 296(2): p. 122132.

309. Planas R., Metz I., Ortiz Y., Vilarrasa N., Jelcic I., Salinas-Riester G., et al., Central role of Th2/Tc2 lymphocytes in pattern II multiple sclerosis lesions. Ann Clin Transl Neurol, 2015. 2(9): p. 875-893.

310. Lafaille J.J., Keere F.V., Hsu A.L., Baron J.L., Haas W., Raine C.S., et al., Myelin basic protein-specific T helper 2 (Th2) cells cause experimental autoimmune encephalomyelitis in immunodeficient hosts rather than protect them from the disease. J Exp Med, 1997. 186(2): p. 307-312.

311. Mucida D., Husain M.M., Muroi S., van Wijk F., Shinnakasu R., Naoe Y., et al., Transcriptional reprogramming of mature CD4(+) helper $\mathrm{T}$ cells generates distinct $\mathrm{MHC}$ class II-restricted cytotoxic T lymphocytes. Nat Immunol, 2013. 14(3): p. 281-289.

312. Peelen E., Thewissen M., Knippenberg S., Smolders J., Muris A.H., Menheere P., et al., Fraction of IL-10+ and IL-17+ CD8 T cells is increased in MS patients in remission and during a relapse, but is not influenced by immune modulators. J Neuroimmunol, 2013. 258(1-2): p. 77-84.

313. Treiber G., Prietl B., Frohlich-Reiterer E., Lechner E., Ribitsch A., Fritsch M., et al., Cholecalciferol supplementation improves suppressive capacity of regulatory T-cells in young patients with new-onset type 1 diabetes mellitus - A randomized clinical trial. Clin Immunol, 2015. 161(2): p. 217-224.

314. Chauhan S.K., Saban D.R., Lee H.K., and Dana $\mathrm{R}$., Levels of Foxp3 in regulatory $\mathrm{T}$ cells reflect their functional status in transplantation. J Immunol, 2009. 182(1): p. 148-153.

315. Niino M., Fukazawa T., Miyazaki Y., Takahashi E., Minami N., Amino I., et al., Suppression of IL-10 production by calcitriol in patients with 
multiple sclerosis. J Neuroimmunol, 2014. 270(1-2): p. 86-94.

316. Bhargava P., Steele S.U., Waubant E., Revirajan N.R., Marcus J., Dembele M., et al., Multiple sclerosis patients have a diminished serologic response to vitamin D supplementation compared to healthy controls. Mult Scler, 2016. 22(6): p. 753-760.

317. Holowaychuk M.K., Birkenheuer A.J., Li J., Marr H., Boll A., and Nordone S.K., Hypocalcemia and hypovitaminosis $D$ in dogs with induced endotoxemia. J Vet Intern Med, 2012. 26(2): p. 244-251.

318. Mehta B.K., New hypotheses on sunlight and the geographic variability of multiple sclerosis prevalence. J Neurol Sci, 2010. 292(1-2): p. 5-10.

319. Farez M.F., Mascanfroni I.D., Mendez-Huergo S.P., Yeste A., Murugaiyan G., Garo L.P., et al., Melatonin Contributes to the Seasonality of Multiple Sclerosis Relapses. Cell, 2015. 162(6): p. 1338-1352.

320. Golan D., Staun-Ram E., Glass-Marmor L., Lavi I., Rozenberg O., Dishon S., et al., The influence of vitamin D supplementation on melatonin status in patients with multiple sclerosis. Brain Behav Immun, 2013. 32: p. 180-185.

321. Gorman S., Black L.J., Feelisch M., Hart P.H., and Weller R., Can skin exposure to sunlight prevent liver inflammation? Nutrients, 2015. 7(5): p. 3219-3239.

322. McCollum E.V., Pitz W., Simmonds N., Becker J.E., Shipley P.G., and Bunting R.W., The effect of additions of fluorine to the diet of the rat on the quality of the teeth. 1925. Studies on experimental rickets. XXI. An experimental demonstration of the existence of a vitamin which promotes calcium deposition. 1922. The effect of additions of fluorine to the diet of the rat on the quality of the teeth. 1925. J Biol Chem, 2002. 277(19): p. E8.

323. Holick M.F., The cutaneous photosynthesis of previtamin D3: a unique photoendocrine system. J Invest Dermatol, 1981. 77(1): p. 5158.

324. Acheson E.D., Bachrach C.A., and Wright F.M., Some comments on the relationship of the distribution of multiple sclerosis to latitude, solar radiation, and other variables. Ac- ta Psychiatr Scand Suppl, 1960. 35(147): p. 132-147.

325. Correale J., If I had clinically isolated syndrome with magnetic resonance imaging diagnostic of multiple sclerosis, I would take vitamin D 10,000 IU daily: Yes. Mult Scler, 2013. 19(2): p. 137-139.

326. Papeix C. and Lubetzki C., If I had a clinically isolated syndrome with MRI diagnostic of MS, I would take vitamin D 10,000 IU daily: No. Mult Scler, 2013. 19(2): p. 140-142.

327. Ascherio A. and Munger K.L., Not too late to take vitamin D supplements. Ann Neurol, 2014. 76(3): p. 321-322.

328. Vitamin D: hope on the horizon for MS prevention? Lancet Neurol, 2010. 9(6): p. 555.

329. Kuhle J., Disanto G., Dobson R., Adiutori R., Bianchi L., Topping J., et al., Conversion from clinically isolated syndrome to multiple sclerosis: A large multicentre study. Mult Scler, 2015. 21(8): p. 1013-1024.

330. Nederlandse Vereniging voor Neurologie., Richltijn Multiple Sclerose 2012: Bohn Stafleu van Loghum, Utrecht, the Netherlands.

331. Lonergan R., Kinsella K., Duggan M., Jordan S., Hutchinson M., and Tubridy N., Discontinuing disease-modifying therapy in progressive multiple sclerosis: can we stop what we have started? Mult Scler, 2009. 15(12): p. 1528-1531.

332. Gupta Konijeti G., Arora P., Boylan M.R., Song Y., Huang S., Harrell F., et al., Vitamin D Supplementation Modulates T Cell-Mediated Immunity in Humans: Results from a Randomized Control Trial. J Clin Endocrinol Metab, 2016. 101(2): p. 533-538.

333. Larsen M., Sauce D., Arnaud L., Fastenackels S., Appay V., and Gorochov G., Evaluating cellular polyfunctionality with a novel polyfunctionality index. PLoS One, 2012. 7(7): p. e42403.

334. Rothhammer V., Heink S., Petermann F., Srivastava R., Claussen M.C., Hemmer B., et al., Th17 lymphocytes traffic to the central nervous system independently of alpha4 integrin expression during EAE. J Exp Med, 2011. 208(12): p. 2465-2476. 
335. Schneider-Hohendorf T., Rossaint J., Mohan H., Boning D., Breuer J., Kuhlmann T., et al., VLA-4 blockade promotes differential routes into human CNS involving PSGL-1 rolling of T cells and MCAM-adhesion of TH17 cells. J Exp Med, 2014. 211(9): p. 1833-1846.

336. Venken K., Hellings N., Thewissen M., Somers V., Hensen K., Rummens J.L., et al., Compromised CD4+ CD25(high) regulatory $\mathrm{T}$-cell function in patients with relapsing-remitting multiple sclerosis is correlated with a reduced frequency of FOXP3-positive cells and reduced FOXP3 expression at the single-cell level. Immunology, 2008. 123(1): p. 79-89.

337. Bandala-Sanchez E., Zhang Y., Reinwald S., Dromey J.A., Lee B.H., Qian J., et al., T cell regulation mediated by interaction of soluble CD52 with the inhibitory receptor Siglec10. Nat Immunol, 2013. 14(7): p. 741-748.

338. Li R., Rezk A., Miyazaki Y., Hilgenberg E., Touil H., Shen P., et al., Proinflammatory GMCSF-producing $B$ cells in multiple sclerosis and B cell depletion therapy. Sci Transl Med, 2015. 7(310): p. 310ra166.

339. Grishkan I.V., Fairchild A.N., Calabresi P.A., and Gocke A.R., 1,25-Dihydroxyvitamin D3 selectively and reversibly impairs $T$ helpercell CNS localization. Proc Natl Acad Sci U S A, 2013. 110(52): p. 21101-21106.

340. Rotstein D.L., Healy B.C., Malik M.T., Carruthers R.L., Musallam A.J., Kivisakk P., et al., Effect of vitamin D on MS activity by disease-modifying therapy class. Neurol Neuroimmunol Neuroinflamm, 2015. 2(6): p. e167.

341. Ahn J., Yu K., Stolzenberg-Solomon R., Simon K.C., McCullough M.L., Gallicchio L., et al., Genome-wide association study of circulating vitamin D levels. Hum Mol Genet, 2010. 19(13): p. 2739-2745.

342. Wang T.J., Zhang F., Richards J.B., Kestenbaum B., van Meurs J.B., Berry D., et al., Common genetic determinants of vitamin $D$ insufficiency: a genome-wide association study. Lancet, 2010. 376(9736): p. 180-188.

343. Harris S.S., Vitamin D and African Americans. J Nutr, 2006. 136(4): p. 1126-1129.

344. Powe C.E., Evans M.K., Wenger J., Zonderman A.B., Berg A.H., Nalls M., et al., Vitamin Dbinding protein and vitamin $D$ status of black Americans and white Americans. N Engl J Med, 2013. 369(21): p. 1991-2000.

345. Ramagopalan S.V., Maugeri N.J., Handunnetthi L., Lincoln M.R., Orton S.M., Dyment D.A., et al., Expression of the multiple sclerosis-associated MHC class II Allele HLADRB1*1501 is regulated by vitamin D. PLoS Genet, 2009. 5(2): p. e1000369.

346. Berge T., Sørum Leikfoss I., Skaara Brorson I., Daniel Bos S., Page C., Gustavsen M.W., et al., The MS susceptibility genes TAGAP and IL2RA are regulated by vitamin D in CD4+ T cells. Genes and Imunity, 2016. 17(2): p. 118127.

347. Farias A.S., Spagnol G.S., Bordeaux-Rego P., Oliveira C.O., Fontana A.G., de Paula R.F., et al., Vitamin D3 induces IDO+ tolerogenic DCs and enhances Treg, reducing the severity of EAE. CNS Neurosci Ther, 2013. 19(4): p. 269-277.

348. Fernandes de Abreu D.A., Landel V., Barnett A.G., McGrath J., Eyles D., and Feron F., Prenatal vitamin $D$ deficiency induces an early and more severe experimental autoimmune encephalomyelitis in the second generation. Int J Mol Sci, 2012. 13(9): p. 10911-10919.

349. Rolf L., Muris A.H., Hupperts R., and Damoiseaux J., Illuminating vitamin D effects on $B$ cells - the multiple sclerosis perspective. Immunology, 2016. 147(3): p. 275-284.

350. Smolders J., Thewissen M., Theunissen R., Peelen E., Knippenberg S., Menheere P., et al., Vitamin D-related gene expression profiles in immune cells of patients with relapsing remitting multiple sclerosis. J Neuroimmunol, 2011. 235(1-2): p. 91-97.

351. Simon K.C., Munger K.L., Xing Y., and Ascherio A., Polymorphisms in vitamin D metabolism related genes and risk of multiple sclerosis. Mult Scler, 2010. 16(2): p. 133-138.

352. Muris A.H., Smolders J., and Damoiseaux J., Comment on the article by Allen et al. 'A pilot study of the immunological effects of high-dose vitamin D in healthy volunteers'. Mult Scler, 2013. 19(4): p. 510.

353. MacCoun R. and Perlmutter S., Blind analysis: Hide results to seek the truth. Nature, 2015. 526(7572): p. 187-189. 
354. Nuzzo R., How scientists fool themselves and how they can stop. Nature, 2015. 526(7572): p. 182-185.

355. Wang Y., Marling S.J., McKnight S.M., Danielson A.L., Severson K.S., and Deluca H.F., Suppression of experimental autoimmune encephalomyelitis by $300-315 \mathrm{~nm}$ ultraviolet light. Arch Biochem Biophys, 2013. 536(1): p. 81-86.

356. Becklund B.R., Severson K.S., Vang S.V., and DeLuca H.F., UV radiation suppresses experimental autoimmune encephalomyelitis independent of vitamin $D$ production. Proc Natl Acad Sci U S A, 2010. 107(14): p. 64186423.

357. Derakhshandi H., Etemadifar M., Feizi A., Abtahi S.H., Minagar A., Abtahi M.A., et al., Preventive effect of vitamin D3 supplementation on conversion of optic neuritis to clinically definite multiple sclerosis: a double blind, randomized, placebo-controlled pilot clinical trial. Acta Neurol Belg, 2013. 113(3): p. 257-263.

358. Malik M.T., Healy B.C., Benson L.A., Kivisakk P., Musallam A., Weiner H.L., et al., Factors associated with recovery from acute optic neuritis in patients with multiple sclerosis. Neurology, 2014. 82(24): p. 2173-2179.

359. Wagner C.L., Greer F.R., American Academy of Pediatrics Section on Breastfeeding, and American Academy of Pediatrics Committee on Nutrition, Prevention of rickets and vitamin D deficiency in infants, children, and adolescents. Pediatrics, 2008. 122(5): p. 11421152.

360. Voedingscentrum,Voedingsnormen en suppletieadviezen vitamine $D$, Voedingscentrum, Eerlijk over eten, 2012. Available from http://www.voedingscentrum.nl/Assets/Uplo ads/Documents/Eten\%20en\%20gezondheid/ Vita-

mines/Basisartikel\%20Voedingsnormen\%20e n\%20suppletieadviezen\%20vitamine\%20D_d ef.pdf

361. Lanting C.I., Heerdink-Obenhuijsen N., Schuit-van Raamsdonk H.L.L., Hofman-van den Hoogen E.M.M., Leeuwenburg-Grijseels E.H., Broerse A., et al., JGZ-richtlijn Voeding en eetgedrag. 2013. Available from https://www.ncj.nl/richtlijnen/jgzrichtlijnenw ebsite/details-richtlijn/?richtlijn=4

362. Health Council the Netherlands, Evaluation of the dietary reference values for vitamin $D$. 2012, Health Council of the Netherlands: The Hague.

363. Carmel N.N., Rotman-Pikielny P., Lavrov A., and Levy Y., Vitamin D Antibodies in Systemic Sclerosis Patients: Findings and Clinical Correlations. Isr Med Assoc J, 2015. 17(2): p. 80-84.

364. Carvalho J.F., Blank M., Kiss E., Tarr T., Amital $H_{\text {., }}$ and Shoenfeld Y., Anti-vitamin D, vitamin $D$ in SLE: preliminary results. Ann N Y Acad Sci, 2007. 1109: p. 550-557.

365. Rinaldi A.O., Sanseverino I., Purificato C., Cortese A., Mechelli R., Francisci S., et al., Increased circulating levels of vitamin $D$ binding protein in MS patients. Toxins (Basel), 2015. 7(1): p. 129-137.

366. Yang M., Qin Z., Zhu Y., Li Y., Qin Y., Jing Y., et al., Vitamin D-binding protein in cerebrospinal fluid is associated with multiple sclerosis progression. Mol Neurobiol, 2013. 47(3): p. 946-956.

367. Smolders J., Peelen E., Thewissen M., Menheere P., Damoiseaux J., and Hupperts R., Circulating vitamin $D$ binding protein levels are not associated with relapses or with vitamin D status in multiple sclerosis. Mult Scler, 2014. 20(4): p. 433-437.

368. Chun R.F., Peercy B.E., Orwoll E.S., Nielson C.M., Adams J.S., and Hewison M., Vitamin D and DBP: the free hormone hypothesis revisited. J Steroid Biochem Mol Biol, 2014. 144 Pt A: p. 132-137.

369. Kleijwegt F.S., Laban S., Duinkerken G., Joosten A.M., Koeleman B.P., Nikolic T., et al., Transfer of regulatory properties from tolerogenic to proinflammatory dendritic cells via induced autoreactive regulatory $\mathrm{T}$ cells. J Immunol, 2011. 187(12): p. 6357-6364.

370. Nikolic T. and Roep B.O., Regulatory multitasking of tolerogenic dendritic cells - lessons taken from vitamin d3-treated tolerogenic dendritic cells. Front Immunol, 2013. 4: p. 113.

371. Debat over de aanpak van chronische ziekten (orginised by the Dutch Society for Immunology). 2014. de Balie. Amsterdam, 
the Netherlands. Available from https://vimeo.com/112430058

372. Dutch Society for Immunology, Nederlandse vereniging voor Immunologie Lustrum debat. 2014, NVVI. Available from https://www.youtube.com/watch?v=2O5UN1 nJ-oM

373. Silberzahn R. and Uhlmann E.L., Crowdsourced research: Many hands make tight work. Nature, 2015. 526(7572): p. 189191.

374. Kleikers P.W., Hooijmans C., Gob E., Langhauser F., Rewell S.S., Radermacher K., et al., A combined pre-clinical meta-analysis and randomized confirmatory trial approach to improve data validity for therapeutic target validation. Sci Rep, 2015. 5: p. 13428.

375. Vinkers C.H., Tijdink J.K., and Otte W.M., Use of positive and negative words in scientific PubMed abstracts between 1974 and 2014: retrospective analysis. BMJ, 2015. 351: p. h6467.

376. Chan A.W., Song F., Vickers A., Jefferson T., Dickersin K., Gotzsche P.C., et al., Increasing value and reducing waste: addressing inaccessible research. Lancet, 2014. 383(9913): p. 257-266.

377. Freedman M.S., Selchen D., Arnold D.L., Prat A., Banwell B., Yeung M., et al., Treatment optimization in MS: Canadian MS Working Group updated recommendations. Can J Neurol Sci, 2013. 40(3): p. 307-323.

378. UK, Multiple Sclerosis Society Top 10 MS research priorities identified. 2013. Available from: https://www.mssociety.org.uk/msnews/2013/09/top-10-ms-researchpriorities-identified

379. van Drooge L. and de Jong S., Valorisatie: onderzoekers dan al veel meer dan ze denken - e-publicatie met voorbeelden en handvatten om zelf valorisatie te organiseren. 2015, Rathenau Instituut: Den Haag. Available from https://www.rathenau.nl/nl/publicatie/valoris atie\%C2\%A0onderzoekers-doen-al-veelmeer-dan-ze-denken

380. Finne H., Day A., Piccaluga A., Spithoven A., Walter $P$., and Wellen D., A composite indi- cator fro knowledge transfer. 2011: Trondheim, Norway.

381. Cijfertool Kosten van Ziekten. Rijksinstituut voor volksgezondheid en milieu: 2011. Bilthoven, the Netherlands. Available from: https://kostenvanziektentool.volksgezondhei denzorg.info/tool/nederlands/

382. Vukusic S. and Confavreux C., Natural history of multiple sclerosis: risk factors and prognostic indicators. Curr Opin Neurol, 2007. 20(3): p. 269-274.

383. Feuillet L., Reuter F., Audoin B., Malikova I., Barrau K., Cherif A.A., et al., Early cognitive impairment in patients with clinically isolated syndrome suggestive of multiple sclerosis. Mult Scler, 2007. 13(1): p. 124-127.

384. Kobelt G., Berg J., Lindgren P., Fredrikson S., and Jonsson B., Costs and quality of life of patients with multiple sclerosis in Europe. J Neurol Neurosurg Psychiatry, 2006. 77(8): p. 918-926.

385. Pfleger C.C., Flachs E.M., and Koch-Henriksen N., Social consequences of multiple sclerosis. Part 2. Divorce and separation: a historical prospective cohort study. Mult Scler, 2010. 16(7): p. 878-882.

386. Cohrs R., Martin T., Ghahramani P., Bidaut L., Higgins P., and Shahzad A., Translational Medicine definition by the European Society for Translational Medicine. 2015. 2(3): p. 8688.

387. Smolders J., Muris A.H., and Damoiseaux J., Immunomodulation by vitamin $\mathrm{D}$ in multiple sclerosis: More than IL-17. J Neuroimmunol, 2016. 292: p. 79-80.

388. Constantinescu C.S., Asher A., Fryze W., Kozubski W., Wagner F., Aram J., et al., Randomized phase $1 b$ trial of MOR103, a human antibody to GM-CSF, in multiple sclerosis. Neurol Neuroimmunol Neuroinflamm, 2015. 2(4): p. e117.

389. The Lancet REWARD (REduce research Waste And Reward Diligence) Campaign. [2016 June]; Available from: http://www.thelancet.com/campaigns/efficie ncy.

390. Science in transition, 2013; Available from: http://www.scienceintransition.nl/overscience-in-transition. 

Addendum 


\section{Curriculum vitae}

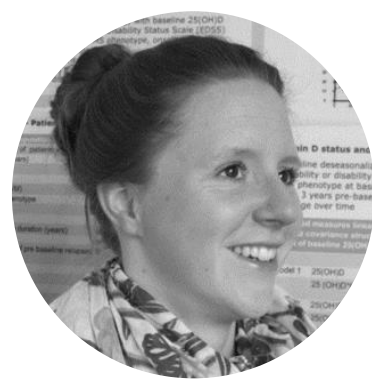

Anne-Hilde Elisabeth Muris was born on May $15^{\text {th }} 1985$ in Utrecht (the Netherlands). She grew up in Bilthoven, where she graduated from secondary school in 2003 (VWO, Het Nieuwe Lyceum). Later that year, she moved to Maastricht to attend medical school (Maastricht University, the Netherlands). During her study she followed the honours programme research and was involved in different research projects at the Maastricht University Medical Centre.

During the final years of her study she became more and more interested in neurology, neuroscience and autoimmunity. After her final clinical internship in neurology, she did a research internship studying the neuromuscular junction in myasthenia gravis (School of Mental Health and Neuroscience, Maastricht University). She graduated from medical school in 2009 and received a Kootstra Fellowship to continue the myasthenia gravis research. In 2010, Anne-Hilde started as a PhD candidate in the vitamin D and multiple sclerosis research group at the Maastricht University Medical Centre and the Academic MS Centre Limburg (Zuyderland Medical Centre - Orbis, Sittard, the Netherlands), were she focussed on the clinical and immunological effects of vitamin $D$ in patients with multiple sclerosis especially after supplementation of high doses of vitamin $D_{3}$. The results of this PhD project are described in this thesis and (parts of it) were also presented at national and international conferences and shared with patients and the general public. Within this period she also followed courses on statistics (longitudinal data analyses), laboratory skills and (clinical) research skills. For the future Anne-Hilde wants to continue to work in the neuro(immuno)logy field as a clinical doctor and translational researcher. 


\section{Publications}

Muris, A.H., Smolders J; Rolf L., Thewissen M., Hupperts R., Damoiseaux, J; Immune regulatory effects of high dose vitamin $D_{3}$ supplementation in a randomised controlled trial in relapsing remitting MS patients receiving IFN- $\beta$; the SOLARIUM study, submitted.

Muris A.H., Smolders J., Rolf L., Klinkenberg L.J.J., van der Linden N., Meex S., Damoiseaux J., Hupperts R.; Vitamin D status does not affect disability progression of MS patients over three year follow-up, PLoS ONE 2016;11(6):e0156122.

Muris A.H., Rolf L., Broen K., Hupperts R., Damoiseaux J., Smolders J.; A low vitamin D status at diagnosis is associated with an early conversion to secondary progressive multiple sclerosis, J Steroid Biochem Mol Biol. 2015 Nov 17. Pii: S0960-0760(15)30136-9.

Muris A.H., Damoiseaux J., Smolders J.; Monitoring in vivo immune modulation by vitamin D in multiple sclerosis; Handbook of Vitamin D in human health: prevention, treatment, and toxicity, Wageningen Academic Publishers, the Netherlands, 2013.

Muris A.H., Damoiseaux J., Smolders J., Cohen Tervaert J.W., Hupperts R., Thewissen M.; Intracellular IL-10 detection in T cells by flow cytometry: the use of protein transport inhibitors revisited; J Immunol Methods. 2012;381:59-65.

Muris A.H., Rolf L., Damoiseaux J., Koeman E., Hupperts R.; Fingolimod in active multiple sclerosis: an impressive decrease in Gd-enhancing lesions. BMC Neurol. 2014; 14: 164.

Muris A.H., Smolders J., Damoiseaux J.; Comment on the article by Allen et al. 'A pilot study of the immunological effects of high-dose vitamin $D$ in healthy volunteers'. Mult Scler. 2013;19:510.

Smolders J., Muris A.H., Damoiseaux J.; Immunomodulation by vitamin D in multiple sclerosis: more than IL-17, J Neuroimmunol. 2016;292:79-80.

Rolf L., Muris A.H., Hupperts R., Damoiseaux J.; Illuminating vitamin D effects on B-cells the multiple sclerosis perspective, Immunology, 2016;147(3):275-84.

Peelen E., Muris A.H., Damoiseaux J., Knippenberg S., Broen K., Smolders J., Cohen Tervaert J.W., Hupperts R., Thewissen M.; GM-CSF production by CD4 ${ }^{+}$T cells in MS patients: regulation by regulatory T cells and vitamin D, J Neuroimmunol. 2015;280:36-42.

Rolf L., Muris A.H., Hupperts R., Damoiseaux J.; Vitamin D effects on B cell function in autoimmunity; Ann N Y Acad Sci. 2014;1317:84-91.

Rolf L., Muris A.H., Damoiseaux J., van Daele M., Hupperts R.; Paroxysmal atrial fibrillation after initiation of fingolimod for multiple sclerosis treatment. Neurology. 2014;82:1008-9. 
Peelen E., Knippenberg S., Muris A.H., Thewissen M., Smolders J., Cohen Tervaert J.W., Hupperts R. Damoiseaux J.; Effects of vitamin D on the peripheral adaptive immune system: a review. Autoimmun Rev. 2011;10:733-43.

Peelen E., Damoiseaux J., Muris A.H., Knippenberg S., Smolders J., Hupperts R., Thewissen M.; Increased inflammasome related gene expression profile in PBMC may facilitate $T$ helper 17 cell induction in multiple sclerosis, Mol Immunol. 2015;63:521-9.

Peelen E., Thewissen M., Knippenberg S., Smolders J., Muris A.H., Menheere P., Cohen Tervaert J.W., Hupperts R., Damoiseaux J.; Fraction of IL- $10^{+}$and $\mathrm{IL}_{-1}-17^{+} \mathrm{CD} 8^{+} \mathrm{T}$ cells is increased in MS patients in remission and during a relapse, but is not influenced by immune modulators, J Neuroimmunol. 2013;258:77-84.

Vrolix K., Niks E.H., Le Panse R., van Ostaijen-Ten Dam M.M., Muris A.H., Jol-van der Zijde C.M., van Tol M.J., Losen M., Molenaar P.C., van Zoelen E.J., Berrih-Aknin S., De Baets M.H., Verschuuren J.J., Martínez-Martínez P.; Reduced thymic expression of ErbB receptors without auto-antibodies against synaptic ErbB in myasthenia gravis. J Neuroimmunol. 2011;232:15865. 


\section{Oral and poster presentations}

Research in this thesis has been presented at:

\section{Scientific conferences}

- "Wetenschappelijk symposium", Zuyderland Medical Centre, Sittard/Heerlen, the Netherlands

- $\quad$ Annual meetings of the Dutch Society of Immunology, the Netherlands

- Belgian Immunological Society Meeting on Autoimmunity, Hasselt, Belgium (2011)

- MS Research Days, the Netherlands

- Intercity, Limburgs Genootschap voor Neurowetenschappen, Stein-Urmond, the Netherlands (2011)

- $\quad$ Research days, School for Mental Health and Neuroscience, Maastricht, the Netherlands

- $\quad 3^{\text {rd }}$ International Conference on Immune Tolerance, Amsterdam, the Netherlands

- $\quad 18^{\text {th }}$ Workshop on Vitamin D, Delft, the Netherlands

- $13^{\text {th }}$ European Society of Neuroimmunology Course, Porto, Portugal

- Congresses of the European Committee for treatment and research in multiple sclerosis (ECTRIMS)

\section{Days for patients and general public:}

- $\quad$ 8-UMC tour, Dutch Society for Immunology, Maastricht, the Netherlands (2014)

- MS goes live, the Netherlands

- $\quad$ Charity runs for research in multiple sclerosis, the Netherlands 


\section{Affiliations of co-authors}

Dr. Kelly Broen

Prof. dr. Jan Willem Cohen Tervaert

Dr. Jan Damoiseaux

Prof. dr. Raymond Hupperts

Dr. Lieke Klinkenberg

Dr. Stephanie Knippenberg

Drs. Noreen van der Linden

Dr. Steven Meex

Dr. Evelyn Peelen

Drs. Linda Rolf
Clinical Chemistry, Zuyderland Medical Centre, Sittard, the Netherlands

Maastricht University, Maastricht, the Netherlands

Central Diagnostic Laboratory, Maastricht University Medical Centre, Maastricht, the Netherlands

Academic MS Centre Limburg, Zuyderland Medical Centre, Sittard, the Netherlands

School for Mental Health and Neuroscience, Maastricht University Medical Centre, Maastricht, the Netherland

Central Diagnostic Laboratory, Maastricht University Medical Centre, Maastricht, the Netherlands

${ }^{*}$ Current address: Clinical Trial Centre Maastricht, Maastricht, the Netherlands

School for Mental Health and Neuroscience, Maastricht University Medical Centre, Maastricht, the Netherlands

Academic MS Centre Limburg, Zuyderland Medical Centre, Sittard, the Netherlands

*Current address: Department of Neurology, Zuyderland Medical Centre, Heerlen, the Netherlands

Central Diagnostic Laboratory, Maastricht University Medical Centre, Maastricht, the Netherlands

Central Diagnostic Laboratory, Maastricht University Medical Centre, Maastricht, the Netherlands

School for Mental Health and Neuroscience, Maastricht University Medical Centre, Maastricht, the Netherlands

Academic MS Centre Limburg, Zuyderland Medical Centre, Sittard, the Netherlands

*Current address: Centre de Recherche du Centre Hospitalier de I'Université de Montreal, Montreal, Canada

School for Mental Health and Neuroscience, Maastricht University Medical Centre, Maastricht, the Netherland

Academic MS Centre Limburg, Zuyderland Medical Centre, Sittard, the Netherlands 
Dr. Joost Smolders

Dr. Marielle Thewissen
Academic MS Centre Limburg, Zuyderland Medical Centre, Sittard, the Netherlands

*Current address: Department of Neurology, Canisius Wilhelmina Hospital, Nijmegen, the Netherlands

Academic MS Centre Limburg, Zuyderland Medical Centre, Sittard, the Netherlands

*Current address: FF Pharma, Diepenbeek, Belgium 


\section{Dankwoord/Acknowledgements}

Hype or hope? Deze vraag is niet alleen van toepassing op de rol van vitamine D in multiple sclerose, maar geldt ook zeker voor het verrichten van wetenschappelijk onderzoek. Hoge verwachtingen, enthousiasme en kleine en grote teleurstellingen vechten afwisselend om een plekje. En of iets nu waar is als de wetenschap het heeft bewezen of als genoeg, of de júíste, mensen erin geloven ..... wetenschap kan in ieder geval alleen beoefend worden mét het geloof, de ervaring, kennis en hulpvaardigheid van heel veel góéde mensen, die samen de bouwstenen vormen van dit werk.

Het feit dat jij dit boekje nu in handen hebt, betekent dat jij nu, in het verleden of in de toekomst, een bijdrage hebt willen en kunnen geven aan dit onderzoek in de brede zin van het woord of aan, zoals het promotiereglement het beschrijft, mijn "proeve van bekwaamheid tot zelfstandig beoefenen van de wetenschap".

Daarom allereerst een persoonlijk bedankje aan jou, voor je bijdrage aan dit werk!

Daarnaast wil ik een aantal personen ook hier nog bedanken.

Het begon in 2010 allemaal met een uitdagende hypothese. Die kwam niet zomaar uit de lucht vallen. Een hoop voorwerk was al verricht door de leden van mijn toekomstige onderzoeksgroep. Jullie waren daarna gepassioneerde begeleiders. Allereerst Raymond Hupperts, bedankt voor je nimmer aflatende vertrouwen, je support, je positieve kijk, je hele korte reactietijd op alle mails en manuscripten en voor alle kansen die je creëerde. Jan Damoiseaux, jouw praktische aanpak, leiderschapskwaliteiten, eerlijkheid en passie voor de immunologie hebben een hele belangrijke bijdrage geleverd aan mijn kijk op de wetenschap en de vooruitgang van ons onderzoek. Fijn dat je deur altijd voor ons als promovendi openstond en -staat! Joost Smolders, jouw eindeloze energie en enthousiasme hebben mij bij onze eerste kennismaking over de streep getrokken, hebben veel input geleverd aan het onderzoek en geven dat nog steeds! En dan Mariëlle Thewissen, dank je wel voor je dagelijkse hulp als vraagbaak in de eerste jaren en je minder frequente, en meer op afstand, maar nog altijd kwalitatieve, bijdrage daarna. Bedankt! 
Om ervaring te delen en te groeien als onderzoeker waren er de medepromovendi. Eigenlijk verdeeld over twee perioden: in "deel 1" allereerst natuurlijk Evelyn en Stephanie, bedankt voor jullie collegialiteit, het delen van alle (eerdere) ervaringen en de gezelligheid! Met daarbij natuurlijk alle oud-collega's van de immunologie en tumorimmunologie.

In "deel 2" krijg jij, Linda de eerste plaats. Als enige directe collega, hebben we samen volop geleerd, beleefd, gefantaseerd, frustraties gedeeld, nagedacht, lekker gegeten en leuke dingen gedaan,..... Heel erg fijn dat jij vanuit het hoge Noorden naar Maastricht bent gekomen en heel veel succes met het afronden van jouw eigen proefschrift! Daarnaast hebben we de laatste jaren nieuwe inzichten, gezelligheid en lunchmomenten gedeeld met de promovendi van het Centraal Diagnostisch Laboratorium, fijn dat we bij jullie mochten aansluiten!

Dan waren er heel veel hulptroepen uit alle richtingen. Maria en Jose, de vliegende keepers op het lab van de immunologie, en dat hebben jullie waargemaakt! Superfijn dat ik altijd met alle vragen bij jullie terecht kon! En bedankt voor de heerlijke vlaaien, de interesse en de gezelligheid.

De analisten in het AzM: Jozien, ook jij staat altijd klaar om vragen te beantwoorden of hulp te bieden! En niet te vergeten Lucienne, Erik, Petra, Nele, Henk en Ruud, en Joyce en Jos.

Dan in Sittard het MS-team: Bertine (mijn liefste koerier!) en het MS-research team, Sandra, Lianne en Maartje, enorm bedankt voor jullie hulp met de SOLAR/SOLARIUM studie. Ook natuurlijk Jolanda, Mariëlle, Judith, Ingrid, Tiny en ook MS-neurologen Fergus Rooyer en Oliver Gerlach, bedankt voor de fijne samenwerking!

Dan heel zakelijk gezegd, de onderzoeksobjecten: er kan heel veel in het laboratorium onderzocht worden maar zonder mensen met of zonder ziekte en enthousiaste neurologen en verpleegkundigen die hen begeleiden is het nauwelijks mogelijk onderzoeksresultaten te vertalen naar de gezondheidszorg. Bij deze dus een grote dank aan iedereen bij wie we voor de studies bloed mochten afnemen en alle patiënten met vertrouwen in de toekomst die bereid waren, en zijn, deel te nemen aan onze studies! Ook heel veel dank aan de neurologen, verpleegkundigen en medewerkers van het lab elders in het land. Bedankt voor het benaderen van patiënten, het regelen van de logistiek en het fijne (meestal telefonische) contact! Drs. Samijn, Dr. Verheul, Dr. Frequin, Ester van der Heiden, Tiny Janssen, Joke van Zoest, Thea Wildschut, Bianca Broere- van der Velde en Kitty Blaauwendraat, van het Maasstadziekenhuis in Rotterdam, het Groene Hart Ziekenhuis in Gouda en het St. Antoniusziekenhuis in Nieuwegein.

Een enthousiaste promovendus is natuurlijk ook niet onbelangrijk, en de vorming daarvan startte eigenlijk al voor het begin van dit promotieonderzoek. Dear Kathleen, Chiara, Alejandro, Marko and Jo, it was really 'een feestje' to work together with you for more than a year during the final year of my study and thereafter. You really introduced me in the PhDstudent life in and outside the University! Thanks for all the help, coffee breaks, nice trips, 
carnival celebrations and so on..... Kathleen, leuk dat jij als mijn oud-stagebegeleidster nu mijn paranimf wil zijn!

En een promovendus blijft enthousiast als zij leuke kamergenoten heeft: in de aanloop van deze promotie, op de eerste verdieping van de UNS 50, natuurlijk Els. Op de $5^{\mathrm{e}}$ verdieping Petra en Mirelle, bedankt voor de hulp, de fijne gesprekken en de gezelligheid!

Dan het enthousiasme van mensen buiten de universiteit: Steven, Lieke en Noreen bedankt voor jullie hulp bij het database paper, Jonathan Alsop thank you for your statistical expertise and input in our 'database paper'. Kelly Broen, Joop ten Kate en Roosmarie Drenth, bedankt voor de samenwerking in het Klinisch Chemisch lab in het Zuyderland/Orbis.

Leergierige studenten leveren een gezellige bijdrage op het lab, helpen met het verzamelen van data, komen met frisse ideeën en kritische vragen om hun begeleider bij de les te houden. Loubna, jij kwam min of meer toevallig bij mij terecht, maar je enthousiasme en inzet was enorm. Dank je wel voor je bijdrage en veel succes met je nieuwe opleiding! Daarna Seneh, dank je wel voor je bijdrage en vrolijkheid! Tom, ook jij bedankt voor je inzet en enthousiasme. Jullie maakten het kleine vitamine D en MS groepje in Maastricht compleet.

Andere randvoorwaarden werden van allerlei kanten geboden: voor het regelwerk zijn een aantal secretaresses en managers onmisbaar. Bedankt Ans! Jitske Beukema, bedankt voor het beantwoorden van vragen over zaken rondom klinische studies en de SOLAR studie.

Bedankt ook voor een bijdrage in de financiering en daarmee het vertrouwen in ons onderzoek: Merck Sereno en het Nationaal MS fonds.

De kwaliteitscontrole van het onderzoek werd versterkt door reviewers van de uit het onderzoek ontstane artikelen, de beoordelingscommissie van dit proefschrift en de medisch ethische commissie Zuyderland Zuyd die het onderzoek goedkeurde. Bedankt voor jullie bijdrage!

Dan leveren aan een ontspannen promovendus natuurlijk ook familie en vrienden een belangrijke bijdrage: op en buiten het werk eerdergenoemde (oud-) collega's, en mijn studievriendinnetjes: Sue Yan (voor jou, als paranimf, én voor mij op 7 oktober de eerste keer!), Rianne, Lonneke, Mirjam en Rianne en Rianne uit het Maastrichtse! Ik hoop dat we samen nog veel lekkers kunnen proeven en leuks kunnen beleven! Dan wil ik ook alle 'schilders' onder leiding van Annemiek voor de (helaas niet altijd wekelijkse) creatieve afleiding bedanken.

Natuurlijk dragen aan afleiding en ontspanning ook de familiale steun uit den lande bij. Vanuit het midden van het land: Elvire (jij begon al vroeg met het verzamelen van stellingen!) en Walter, Vincent (bedankt voor alle gezelligheid en onze broer-zus uitjes) en Lieneke. Vanuit het "Brabantse": Jos en Jacqueline, Fenna en Huib, en Jasper. En op de feestdagen en zeker ook via veel kaartjes, de ("Vandebergse") tante's. En natuurlijk Wouter, bedankt voor je 
geduld en steun. Als mede-promovendus, mede-wereldreiziger en sinds dit jaar dan toch ook mede-huisgenoot hebben we al heel wat ervaringen gedeeld. Op naar alle volgende belevenissen!

Natuurlijk valt niet iedereen in een hokje te plaatsen. Er waren ook nog vele anderen met wie ik kortdurend of minder intensief heb samengewerkt: MS collega's uit Hasselt, de collega's van de pathologie (bedankt voor het soepele schema rond het FACS apparaat!), andere collega's van de MHeNS en het CDL ... en hen die ik ondanks alles vergeten ben te noemen.

Heel veel plezier en succes in de werkzaamheden voor de toekomst, ik hoop dat we elkaar nog vaak tegen gaan komen en nogmaals dank jullie wel! 




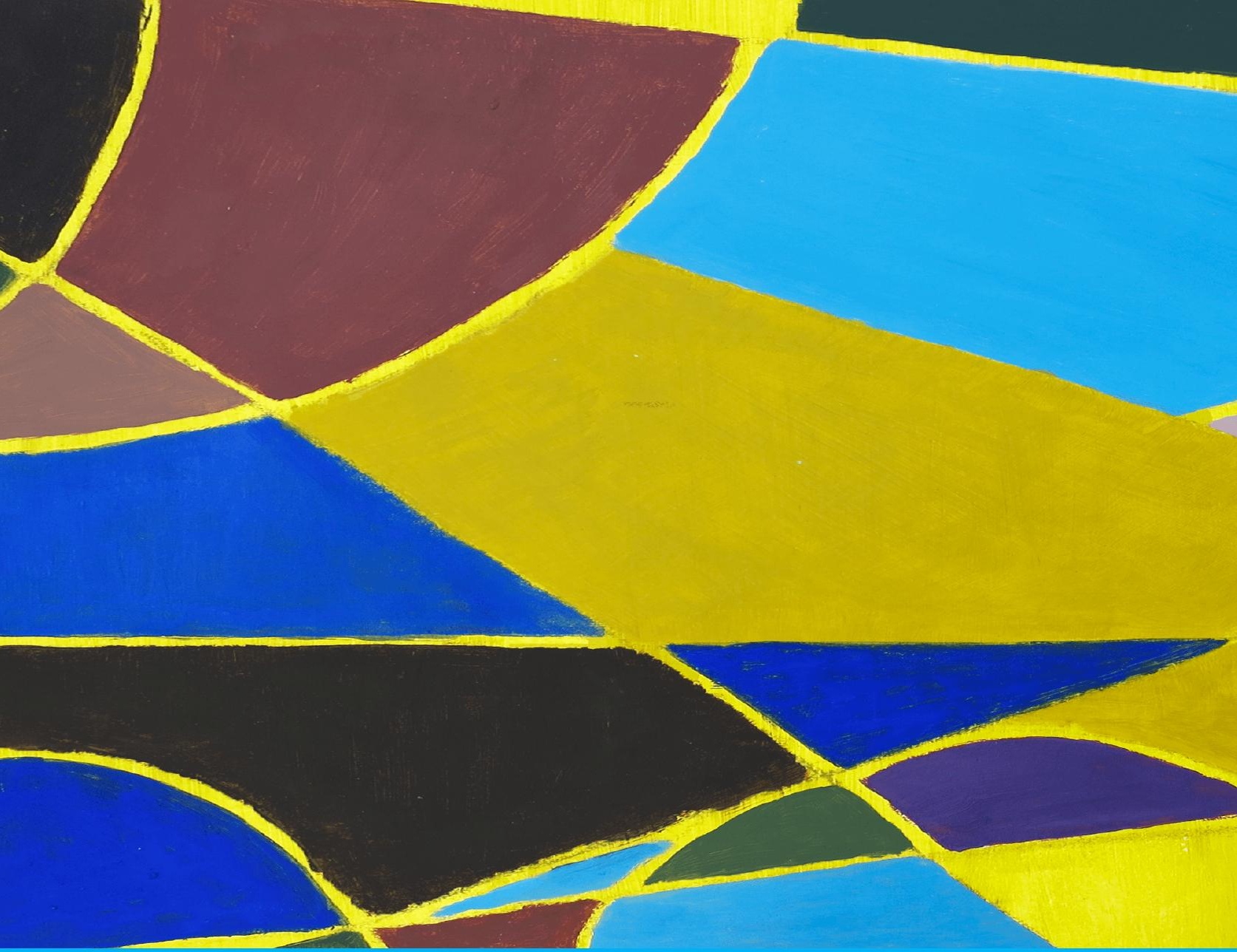

Vitamin D and its role in the disease pathology of multiple sclerosis and many other diseases has gained a lot of attention over the last decades. But is vitamin D really a hope for patients with multiple sclerosis, or have the enthusiasm and high expectations of many people, hyped those hopes up?

The research described in this thesis aims to add scientific evidence to the role of vitamin D in multiple sclerosis. It increases our scientific understanding of the role of vitamin $\mathrm{D}$ in the disease progression. Furthermore, the immune regulatory effects of high dose vitamin D supplementation as a possible add on therapy in patients with relapsing remitting multiple sclerosis were investigated. 